\title{
Unified MIMO-Multicarrier Designs: A Space-Time Shift Keying Approach
}

\author{
Mohammad Ismat Kadir, Shinya Sugiura, Senior Member, IEEE, \\ Sheng Chen, Fellow, IEEE, and Lajos Hanzo, Fellow, IEEE
}

\begin{abstract}
A survey and tutorial is provided on the subject of multiple-input-multiple-output (MIMO) multicarrier (MC) systems relying on the space-time shift keying (STSK) concept. We commence with a brief review of the family of MIMO systems, which is followed by the design of STSK systems in the context of MC modulation-based transmissions over dispersive wireless channels. Specifically, the STSK scheme is amalgamated with orthogonal frequency-division multiplexing, MC code-division multiple access, orthogonal frequency-division multiple access (OFDMA), and single-carrier frequency-division multiple access. We also provide a rudimentary introduction to MC differential STSK employing both conventional differential detection and multiple-symbol differential sphere decoding for the sake of dispensing with channel estimation. We conclude with the design recipes of coherent versus noncoherent MC STSK schemes, complemented by a range of future research ideas.
\end{abstract}

Index Terms-Multiple-input multiple-output (MIMO), linear dispersion code (LDC), spatial modulation (SM), space-time shift keying (STSK), orthogonal frequency-division multiplexing (OFDM), multicarrier code-division multiple access (MC-CDMA), orthogonal frequency-division multiple access (OFDMA), singlecarrier frequency-division multiple access (SC-FDMA).

\section{INTRODUCTION}

$\mathbf{M}$ ULTIPLE-input-multiple-output (MIMO) wireless communication systems have attracted substantial research interests over the last two decades, mainly as a benefit of their capability of providing reliable high-throughput communications without extending the bandwidth of a single-antenna system. Taking the explosive expansion of the Internet and the dramatically increased demand for high-speed multimedia wireless systems into consideration, designing flexible and spectrally efficient transceivers is essential. The

Manuscript received January 30, 2014; revised September 19, 2014; accepted November 17, 2014. This work was supported in part by the Commonwealth Scholarship Commission in the U.K., by RC-UK under the auspices of the IUATC, by EU under the CONCERTO project, and by the European Research Council under its Advanced Fellow Grant.

M. I. Kadir was with the School of Electronics and Computer Science, University of Southampton, Southampton SO17 1BJ, U.K. He is now with the Electronics and Communication Engineering Discipline, Khulna University, Khulna 9208, Bangladesh (e-mail: ismat.kadir@ece.ku.ac.bd).

S. Sugiura is with the Department of Computer and Information Sciences, Tokyo University of Agriculture and Technology, Tokyo 184-8588, Japan (e-mail: sugiura@ieee.org).

S. Chen and L. Hanzo are with the School of Electronics and Computer Science, University of Southampton, Southampton SO17 1BJ, U.K. (e-mail: sqc@ecs.soton.ac.uk; 1h@ecs.soton.ac.uk).

Color versions of one or more of the figures in this paper are available online at http://ieeexplore.ieee.org.

Digital Object Identifier 10.1109/COMST.2014.2375321 throughput of a MIMO system may be increased linearly with the number of antennas [1], [2]. Apart from this multiplexing gain, MIMOs are also capable of benefiting from a spatial diversity gain that is attained by the spatially separated antennas in a dense multipath scattering environment, provided that the antenna-elements are sufficiently far apart for the sake of experiencing independent fading. Thus MIMO systems have the potential of improving both the spectral efficiency and the link reliability of future wireless communications systems.

The employment of multiple antenna elements (AEs) may be expected to exhibit flexibility in terms of striking a compelling compromise between the attainable multiplexing versus diversity gains. However, there exists a fundamental limitation, which is quantified in terms of the diversity-multiplexing tradeoff (DMT) [3]. Motivated by this constraint, linear dispersion codes (LDCs) were proposed in [4], [5] for striking a flexible DMT. As their benefit, LDCs constitute a generic family, which subsumes both the family of diversity and multiplexing schemes, whilst potentially outperforming both-albeit, at the cost of a substantially increased decoding complexity. On the other hand, the concepts of spatial modulation (SM) [6] as well as space shift keying (SSK) [7] emerged as a low-complexity design alternative. The SM/SSK schemes adopted the principle of shrewd transmit antenna (TA) activation for attaining an increased bandwidth efficiency. These low-complexity schemes are not, however, capable of attaining beneficial transmit diversity. Motivated by these conflicting design ideas, space-time shift keying (STSK) [8]-[10] was proposed, which extends the concept of pure spatial-domain TA activation of the SM/SSK schemes to both the spatial and temporal dimensions. The STSK system can thus provide substantial diversity- as well as multiplexing gains. However, the majority of STSK studies were focused on non-dispersive, i.e., narrowband propagation scenarios, rather than on realistic wideband scenarios. Against this background, multicarrier (MC) STSK was conceived in [11]-[13] for facilitating STSK based transmissions over dispersive wireless channels. A particularly promising candidate for the next generation fixed and mobile wireless systems is the combination of STSK techniques with the family of MC communications systems.

Realistic wireless channels are dispersive by nature. Dispersive MIMO channels potentially exhibit frequency diversity, provided that the channel's delay-spread is sufficiently high for ensuring that the subcarriers of a MC system fade near-independently. Alternatively, this phenomenon may also be viewed as the independent fading of the channel impulse response (CIR) taps. A MC system effectively converts the 
dispersive wideband channels into a number of low-rate parallel narrowband frequency-flat subchannels. The unmodulated subcarriers are required to maintain a minimum frequency separation in order to remain orthogonal, but the modulated signal spectra corresponding to the different subcarriers may overlap in the frequency-domain (FD). Hence, the available spectrum can be efficiently utilized. Furthermore, MC systems are capable of significantly reducing the receiver's complexity in broadband channels with the aid of using single-tap FD equalization. Thus MIMO systems combined with MC communications appear to be a promising solution for broadband wireless communications systems. Motivated by the flexible DMT provided by the STSK scheme at a low decoding complexity and by the benefits of MIMO-MC configurations, a number of MC STSK schemes were proposed in [11]-[14]. Specifically, STSK schemes employing orthogonal frequency division multiplexing (OFDM) [11], MC code division multiple access (MCCDMA) [12], orthogonal frequency division multiple access (OFDMA) or single-carrier frequency division multiple access (SC-FDMA) [13] were developed.

MIMO systems relying on MC STSK [11], [13] are capable of ensuring link reliability for transmission over realistic dispersive channels, while simultaneously providing a multiplexing gain at a low decoding complexity. However, the AEs of a co-located STSK system typically suffer from spatially correlated large-scale fading imposed by the ubiquitous shadowing effects [15]. For the sake of mitigating this correlation-induced diversity-gain erosion, the concept of cooperative space-time processing [16], [17] has become popular in recent years, because they benefit from the geographically distributed nature of relay nodes (RNs), where the relays may be viewed as the distributed elements of a MIMO system. As a result, each virtual antenna array (VAA) element experiences uncorrelated fading. Additionally, this cooperative MIMO architecture enables us to avoid the physical limitations of installing multiple antennas on a shirt-pocket sized mobile handset. The concept of cooperative STSK [18] was conceived for frequency-flat Rayleigh fading channels in order to benefit from mobile station (MS)cooperation, although naturally, this scheme suffers from the usual throughput loss imposed by the half-duplex relaying strategy employed. The introduction of successive relaying (SR) [19], on the other hand, is potentially capable of recovering the half-duplex multiplexing loss and hence it was successfully used in [20] as a cooperative space-time coding architecture.

Motivated by these insights, a novel cooperative MC STSK arrangement was conceived in [14] for the sake of benefiting from cooperative diversity [21]. In [14], SR was invoked for mitigating the typical $50 \%$ throughput loss of conventional half-duplex relaying schemes, while MC-CDMA is used for circumventing the dispersive effects of wireless channels as well as for reducing the SR-induced interferences. In short, the STSK systems may be classified as shown in Fig. 1. In this tutorial, we will provide design guidelines for OFDM-aided STSK, MC-CDMA aided STSK, OFDMA/SC-FDMA aided STSK, SR aided distributed MC coherent STSK (CSTSK) and differential STSK (DSTSK) using multiple-symbol differential sphere decoding (MSDSD) for communications over dispersive channels.

\section{STSK Systems}

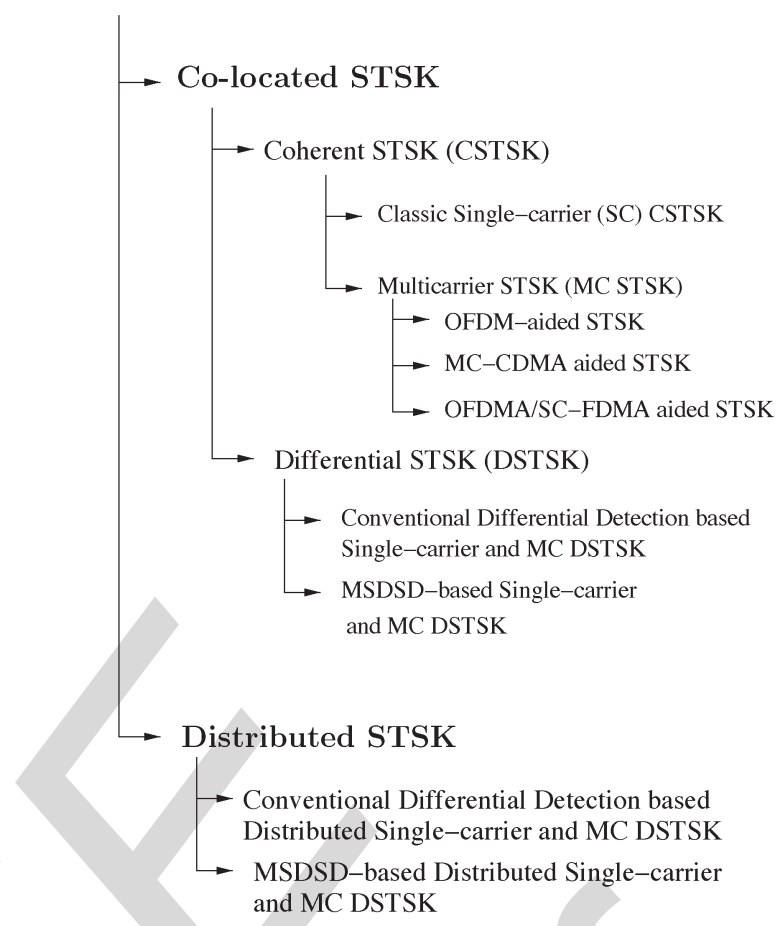

Fig. 1. STSK systems so far studied.

Following a brief review of MIMO systems, of MC systems and of MIMO-MC systems, we will embark on a tutorial on the subject of the family of both co-located and distributed MC STSK systems.

\section{A. Outline}

The remainder of this paper is organized as follows. In Section II, we provide a brief review of the major contributions to the different types of MIMO systems and of the recent design philosophies, which motivated the conception of STSK systems. In Section III, MIMO-OFDM designs are briefly reviewed and the concept of OFDM-aided STSK is described. An overview of multiuser STSK schemes employing MCCDMA and OFDMA/SC-FDMA is provided in Section IV, while distributed MC STSK employing SR is highlighted in Section V. In Section VI, both the hard-decision based maximum likelihood (ML)-MSDSD and the soft-decision based maximum a posteriori (MAP)-MSDSD are invoked for differentially encoded and noncoherently detected OFDM-aided STSK schemes. Section VII provides a range of design guidelines and future research ideas. Finally, Section VIII concludes this treatise.

\section{B. Notations}

The following notations are employed throughout this contribution. Upper-case boldface letter, for example, $\boldsymbol{A}$ denotes a matrix, whereas $\boldsymbol{A}^{T}, \boldsymbol{A}^{H}, \operatorname{tr}(\boldsymbol{A}), \operatorname{vec}(\boldsymbol{A})$, and $\|\boldsymbol{A}\|$ denote the matrix transpose, the Hermitian transpose, the trace, the vectorial stacking operator and the Euclidean norm of $\boldsymbol{A}$ respectively. The operator $|a|$ denotes the complex norm of the complex number $a, \otimes$ the Kronecker product, $\boldsymbol{I}_{T}$ the $(T \times T)$-element 


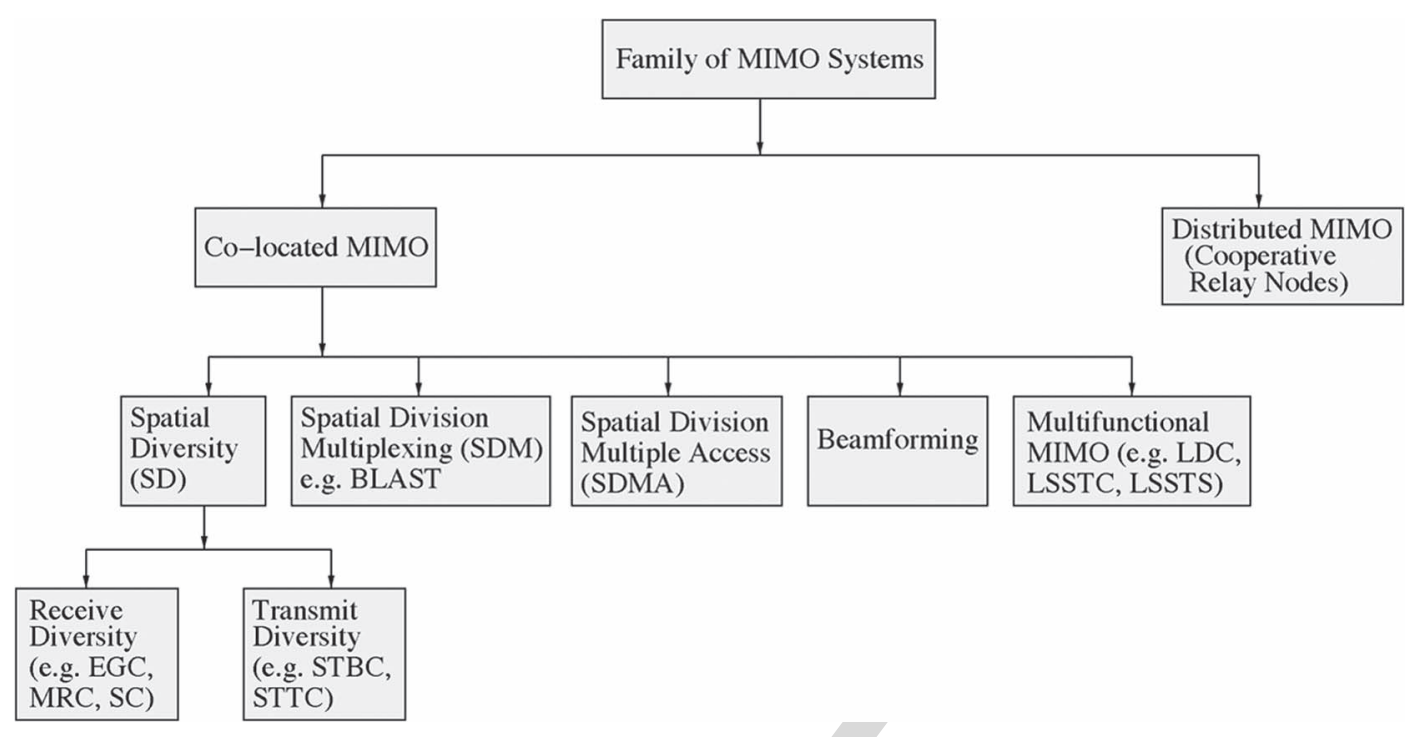

Fig. 2. The simplified family tree of MIMO systems.

identity matrix, $\mathbf{0}_{M \times T}$ the $(M \times T)$-element zero matrix and $\mathfrak{R}(\cdot)$ and $\mathfrak{I}(\cdot)$ the real and the imaginary parts of a complex number, respectively. The notation $\operatorname{diag}\left\{x_{1}, \ldots, x_{N}\right\}$ denotes a diagonal matrix with $x_{1}, \ldots, x_{N}$ on its main diagonal, while $\delta(\cdot)$, $\delta[\cdot]$ and $P(\cdot)$ represent the continuous-time Dirac delta function, the discrete-time Dirac delta function and the probability of ".," respectively. The $N_{c}$-point discrete Fourier transform (DFT) of the symbol stream "•" and the $N_{c}$-point inverse discrete Fourier transform (IDFT) of "•" are denoted by $\mathrm{DFT}_{N_{c}}\{\bullet\}$ and IDFT $_{N_{c}}\{\bullet\}$, respectively. Furthermore, the notation $u$ denotes the generalized user, whereas $v$ refers to the desired user.

\section{MiMO Systems: StATE-OF-THE-ART CONTRIBUTIONS AND STSK}

In this section, we would like to commence with a brief review of the major contributions to the MIMO family, followed by an outline of the recent MIMO techniques, which motivated the invention of the STSK concept.

\section{A. Contributions to Different MIMO Types}

MIMO systems may be classified into different categories [38], [39], as shown in the family tree of Fig. 2, according to the applications they are employed in. The state-of-the-art contributions on different MIMO schemes are summarized in Table I.

Spatial diversity is used in wireless communications systems for combating the deleterious effects of small scale fading and thereby for improving the link's reliability. Diversity combining schemes employed in the receiver, such as equal gain combining (EGC), selection combining (SC) and maximum ratio combining (MRC) can be traced back to the 1950s with reference to Brennan's excellent paper [22]. In order to facilitate diversity-aided transmissions, a simple but witty transmit diversity technique is constituted by Alamouti's scheme [24], which inspired a spate of transmit diversity ideas, as proposed in Tarokh's space-time block code (STBC) and space-time trellis code (STTC) [25], [26].
MIMO systems may be designed for attaining an improved bandwidth efficiency by transmitting different signal streams independently over each of the transmit antennas and are known as spatial division multiplexing (SDM). SDM was first conceived by Paulraj and Kailath [29], who patented the "Distributed Transmit—Directional Receive (DTDR)" concept. Later, Foschini [30] introduced the SDM concept in 1996 under the terminology of Bell Laboratories space-time (BLAST) codes, which later evolved to a modified architecture termed as the vertical BLAST or V-BLAST arrangement proposed by Wolniansky in 1998 [31]. The discovery that for a fixed radiated power and bandwidth the capacity increases linearly with the number of transmit-receive antenna pairs was further supported by Telatar in 1999 [2].

Space-division multiple access (SDMA) employs multiple antennas for the sake of supporting multiple users [34], [40]. Like SDM, SDMA allows us to increase the capacity of a mobile cellular system by exploiting the spatial separation of the users. The spatial gain advantages of SDMA are attained assuming the existence of numerous users having uncorrelated CIRs.

Beamforming architectures are specifically designed for reducing the interference levels with the aid of creating angularly selective beams [33], [41], [42]. Typically, half-wavelengthspaced antenna elements are used for creating a spatially selective transmitter/receiver beam.

There are other types of MIMO systems which are capable of combining the benefits of several MIMO types and may be applied for simultaneously attaining the benefits of having diversity, multiplexing, multiple access and/or beamforming gains. These schemes are referred to as multifunctional MIMO systems [38]. Examples of multifunctional MIMOs include the layered steered space-time codes (LSSTCs) of [37] and the layered steered space-time spreading (LSSTS) of [43].

\section{B. Recent Developments in MIMO Techniques}

In contrast to the classic MIMOs described above, recently a number of MIMO techniques were developed which motivated the STSK concept. Explicitly, the chronological development of 
TABLE I

MAJOR CONTRIBUTIONS CONCEIVED FOR DIFFERENT MIMO APPLICATIONS

\begin{tabular}{|c|c|c|c|}
\hline Category & Year & Author(s) & Contribution \\
\hline \multirow{7}{*}{ Diversity } & 1959 & Brennan [22] & Introduced and analyzed EGC, SC and MRC. \\
\hline & 1994 & $\begin{array}{l}\text { Seshadri and Win- } \\
\text { ters [23] }\end{array}$ & $\begin{array}{l}\text { A transmit diversity scheme was inspired by Wittneben's delay } \\
\text { diversity design. }\end{array}$ \\
\hline & \multirow{3}{*}{1998} & Alamouti [24] & Proposed a transmit diversity scheme relying on two antennas. \\
\hline & & Tarokh et al. [25] & $\begin{array}{l}\text { Conceived space-time block codes based on orthogonal de- } \\
\text { signs. }\end{array}$ \\
\hline & & Tarokh et al. [26] & $\begin{array}{l}\text { Provided design criteria for achieving the maximum attainable } \\
\text { diversity and coding gain. }\end{array}$ \\
\hline & \multirow{2}{*}{2000} & Hughes [27] & Introduced a new DSTBC design based on group codes. \\
\hline & & $\begin{array}{l}\text { Jafarkhani et } \\
\text { al. }[28]\end{array}$ & $\begin{array}{l}\text { Proposed a differential detection scheme for transmit diversity } \\
\text { arrangements. }\end{array}$ \\
\hline \multirow{4}{*}{ Multiplexing } & 1994 & $\begin{array}{ll}\text { Paulraj and } \\
\text { Kailath [29] }\end{array}$ & $\begin{array}{l}\text { Patented the first spatial multiplexing scheme conceived for } \\
\text { broadcast applications. }\end{array}$ \\
\hline & 1996 & Foschini [30] & $\begin{array}{l}\text { Conceived the encoding and decoding of the diagonal BLAST } \\
\text { structure. }\end{array}$ \\
\hline & 1998 & $\begin{array}{l}\text { Wolniansky } \\
\text { al. [31] }\end{array}$ & $\begin{array}{l}\text { The V-BLAST architecture was introduced for reducing com- } \\
\text { plexity in implementing Foscini's approach [30]. }\end{array}$ \\
\hline & 1999 & Telatar [2] & Quantified the MIMO channel capacity. \\
\hline \multirow[t]{2}{*}{ Beamforming } & 1994 & $\begin{array}{ll}\text { Winters } & \text { and } \\
\text { Salz [32] }\end{array}$ & $\begin{array}{l}\text { The impact of diversity of directional transmission on the } \\
\text { capacity was studied. }\end{array}$ \\
\hline & 1997 & Godara [33] & $\begin{array}{l}\text { Antenna arrays were proposed for mobile communications and } \\
\text { their performance was studied. }\end{array}$ \\
\hline \multirow[t]{2}{*}{ SDMA } & 1996 & $\begin{array}{l}\text { Cooper and Gold- } \\
\text { burg [34] }\end{array}$ & Introduced spatial division multiple access. \\
\hline & 2000 & $\begin{array}{l}\text { Vandenameele et } \\
\text { al. [35] }\end{array}$ & Amalgamated OFDM with SDMA. \\
\hline \multirow{4}{*}{ Multifunctional } & \multirow[t]{2}{*}{2002} & $\begin{array}{l}\text { Hassibi and } \\
\text { Hochwald [4] }\end{array}$ & $\begin{array}{l}\text { LDCs were introduced for achieving a flexible diversity- } \\
\text { multiplexing tradeoff. }\end{array}$ \\
\hline & & Heath \& Paulraj [5] & $\begin{array}{l}\text { Improved LDC designs based on frame theoretic matrix lin- } \\
\text { earization were advocated. }\end{array}$ \\
\hline & 2004 & Kim and Chun [36] & $\begin{array}{l}\text { Proposed a MIMO structure, which combines spatial multi- } \\
\text { plexing and beamforming. }\end{array}$ \\
\hline & 2009 & El-Hajjar et al. [37] & Proposed layered steered space-time codes. \\
\hline
\end{tabular}

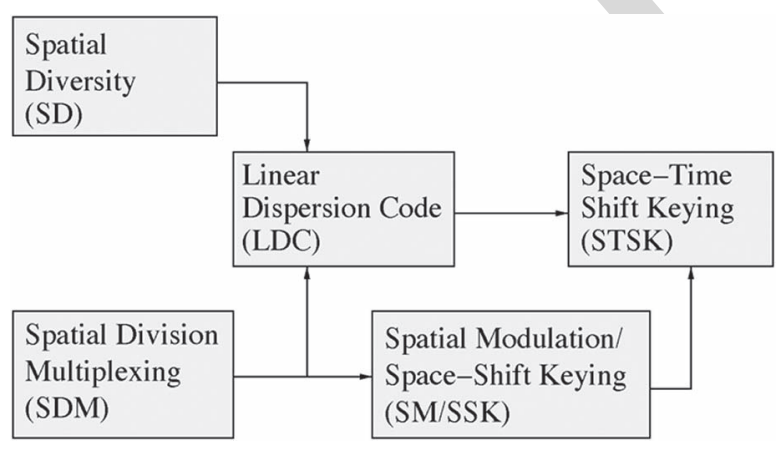

Fig. 3. Development of the STSK concept for striking a flexible DMT.

the MIMO family, which paved the way for the STSK concept is illustrated in Fig. 3 and the major contributions related to SM, SSK and STSK are summarized in Table II.

To be specific, MIMO systems are not expected to be employed for attaining either entirely diversity or solely for multiplexing gain. Rather, in order to strike a flexible DMT
[3], Hassibi and Hochwald [4] proposed LDCs, which were extended by Heath and Paulraj [5] based on frame theory. The flexible benefits of LDC schemes are, however, attained at the cost of imposing high decoding complexity. SM and SSK, on the other hand, are low-complexity solutions which are not capable of providing transmit diversity.

As a predecessor of the SM/SSK/STSK concept, the "space modulation" philosophy which allocates source bits to AEs by simultaneously activating multiple AEs was conceived by Chau and $\mathrm{Yu}$ [44] in 2001. Later, Mesleh et al. [6] introduced the concept of SM for activating only one of the transmit antennas at a time, where the antenna index itself carries additional information, thus attaining an increased throughput. This development was followed by Jeganathan et al. [7], who simply used the presence or absence of energy to transmit information without using any specific signal constellation, which led to the concept of SSK. The SM/SSK [6], [7] scheme employs the antenna index for achieving a multiplexing gain, but owing to activating a single $\mathrm{AE}$, it is incapable of providing a transmit diversity gain. Hence, by intrinsically amalgamating the 
TABLE II

MAJOR CONTRIBUTIONS ON SM/SSK/STSK

\begin{tabular}{|c|c|c|}
\hline Year & Author(s) & Contribution \\
\hline 2001 & Chau and Yu [44] & $\begin{array}{l}\text { Conceived the 'space modulation' philosophy, which allocates source bits to AEs } \\
\text { and simultaneously activates multiple AEs. }\end{array}$ \\
\hline 2006 & Mesleh et al. [45] & $\begin{array}{l}\text { Proposed spatial modulation (SM) for low-complexity spectral efficiency enhance- } \\
\text { ment. }\end{array}$ \\
\hline \multirow{2}{*}{2008} & Mesleh et al. [6] & Presented an analytical investigation of SM. \\
\hline & Mesleh et al. [46] & Analyzed the symbol-error-ratio (SER) of SM. \\
\hline \multirow{2}{*}{2009} & Jeganathan et al. [7] & Conceived space shift keying (SSK). \\
\hline & Jeganathan et al. [47] & $\begin{array}{l}\text { Studied SM as well as SSK and proposed a single-stream optimal ML detection } \\
\text { algorithm. }\end{array}$ \\
\hline \multirow{3}{*}{2010} & Sugiura et al. [8] & Introduced both coherent and differential STSK based on LDCs. \\
\hline & Sugiura et al. [48] & Conceived reduced-complexity detectors for QAM-aided STSK schemes. \\
\hline & Mesleh et al. [49] & Trellis coded SM was introduced. \\
\hline \multirow{8}{*}{2011} & Renzo and Haas [50] & $\begin{array}{l}\text { Studied the performance of SSK-MIMO and proposes a novel SSK-MIMO for } \\
\text { increasing diversity order. }\end{array}$ \\
\hline & Rajashekhar et al. [51] & Proposed a novel DM design for CSTSK based on field extension codes. \\
\hline & Basar et al. [52] & Designed a trellis code for SM. \\
\hline & Yang et al. [53] & $\begin{array}{l}\text { Adaptive SM was proposed by adaptively selecting the modulation order for } \\
\text { improving system performance and for reducing its complexity. }\end{array}$ \\
\hline & Sugiura et al. [9] & Introduced a generalized space-time shift keying arrangement. \\
\hline & Ngo et al. [54] & $\begin{array}{l}\text { Introduced the concept of space-time-frequency shift keying (STFSK) for wideband } \\
\text { channels. }\end{array}$ \\
\hline & Sugiura et al. [18] & Proposed both coherent and noncoherent cooperative STSK. \\
\hline & Renzo et al. [55] & Presented a comprehensive survey of the SM-MIMO scheme. \\
\hline \multirow{8}{*}{2012} & Sugiura et al. $[10]$ & Provided a survey of STSK as a universal MIMO space-time architecture. \\
\hline & $\begin{array}{l}\text { Som and } \\
\text { Chockalingam [56] }\end{array}$ & $\begin{array}{l}\text { Studied SM and SSK in cyclic prefixed single-carrier systems communicating over } \\
\text { dispersive MIMO channels. }\end{array}$ \\
\hline & Basar et al. [57] & $\begin{array}{l}\text { A new class of space-time trellis codes, namely 'super-orthogonal' trellis coded SM } \\
\text { was proposed to achieve maximal diversity and coding gains. }\end{array}$ \\
\hline & Yang et al. [58] & An improved matched-filter detection algorithm was proposed for STSK. \\
\hline & Babich et al. [59] & $\begin{array}{l}\text { Genetic Algorithm based DM optimization was proposed for concatenated coding } \\
\text { assisted STSK. }\end{array}$ \\
\hline & Driusso et al. [11] & Proposed OFDM-aided STSK for dispersive downlink channels. \\
\hline & Rajashekhar et al. [60] & Structured DMs were designed for STSK. \\
\hline & Ngo et al. [61] & $\begin{array}{l}\text { The area spectral efficiency (ASE) of STFSK aided slow-frequency-hopping multiple } \\
\text { access (SFHMA) was investigated. }\end{array}$ \\
\hline \multirow{7}{*}{2013} & Zhang et al. [62] & $\begin{array}{l}\text { A cooperative decode- and forward (DF) DSTSK scheme was proposed employing } \\
\text { MSDSD and multiuser DS-CDMA. }\end{array}$ \\
\hline & Renzo and Haas [63] & Designed SM making provision for achieving transmit diversity gain. \\
\hline & Rajashekhar et al. [64] & Novel TA selection techniques were conceived for SM systems. \\
\hline & $\begin{array}{l}\text { Serafimovski et } \\
\text { al. }[65]\end{array}$ & First practical testbed implementation of SM in the laboratory. \\
\hline & $\begin{array}{ll}\text { Sugiura } & \text { and } \\
\text { Hanzo [66] } & \end{array}$ & Joint DM and constellation optimization was proposed for STSK. \\
\hline & Xu et al. [67] & $\begin{array}{l}\text { Proposed reduced-complexity detection techniques for both SM as well as for STSK } \\
\text { without sacrificing their performance. }\end{array}$ \\
\hline & Kadir et al. [12]-[14] & $\begin{array}{l}\text { Proposed a suite of MC-CDMA- and OFDMA/SC-FDMA-aided co-located and } \\
\text { distributed STSK schemes for dispersive channels. }\end{array}$ \\
\hline \multirow{3}{*}{2014} & Yang et al. [68] & Provided design guidelines for SM-MIMO. \\
\hline & Kadir et al. [69] & Proposed a MC DSTSK scheme employing iterative soft MSDSD. \\
\hline & Renzo et al. [70] & $\begin{array}{l}\text { Presented a review as well as experimental results on SM and discussed its beneficial } \\
\text { application areas. }\end{array}$ \\
\hline
\end{tabular}

concept of LDCs and of SM [6] as well as SSK [7], Sugiura et al. proposed the design philosophy of STSK [8].

1) $S M: \mathrm{SM}$ maps the symbols to the activated transmit antenna index, as an implicit means of conveying extra information. Hence it maps a block of information bits to two information carrying units: to a classic symbol chosen from the constellation and to the unique index of the activated transmit antenna.

The exploitation of the transmit antenna index as an information-bearing unit improves the overall spectral efficiency by $\log _{2} M$ bits, where $M$ denotes the number of transmit antennas. 
The number of bits transmitted using an $\mathcal{L}$-ary constellation equals to $\log _{2} \mathcal{L}$, whereas that using the activated antenna index will be $\log _{2} M$. The SM scheme is capable of transmitting $R$ bits, where $R$ is given by

$$
R=\log _{2}(M \cdot \mathcal{L}),
$$

The most attractive feature of the SM scheme is that it imposes a substantially reduced decoding complexity in comparison to its SDM counterpart.

Since the SM scheme activates a single AE at any signaling interval, only a single radio-frequency (RF) chain is necessitated. Furthermore, the SM scheme is capable of operating using only loose synchronization among the AEs, i.e., it is robust to inter-antenna synchronization (IAS) errors.

2) SSK: In case of SSK aided transmission, the classic PSK/QAM constellation signaling of the SM scheme is deactivated and only the presence or absence of energy is detected at the receiver antenna [7], which facilitates an extremely lowcomplexity decoding.

\section{STSK}

Motivated by the SM/SSK idea of activating a single AE during each symbol durations while mapping source bits to the index of the AE activated, the STSK transmitter activates one out of $Q$ LDC-like dispersion matrices (DMs) $\boldsymbol{A}_{q} \in C^{M \times T}(q=$ $1,2, \ldots, Q)$, which are pre-assigned before transmission. To expound a little further, the DMs or the spreading matrices, as used both in LDC and in STSK, are $(M \times T)$-element matrices of complex numbers, which are generated by optimizing a specific objective function (OF) under a power constraint criterion and are utilized to disperse the source information in both the spatial and time domain [4], [5], [8]. LDCs use a matrix modulation framework [5], where the transmitted codeword is formed by a linear combination of the DMs weighted by the $\mathcal{L}$-PSK/QAM symbols. To be more specific, the LDC transmit codeword $\boldsymbol{X}^{\mathrm{LDC}}[i] \in \mathrm{C}^{M \times T}$ during any signaling interval $i$ corresponding to the symbols $s_{1}[i], s_{2}[i], \ldots, s_{Q}[i]$ and to the DMs

$$
\begin{gathered}
\boldsymbol{A}_{1}=\left[\begin{array}{ccc}
a_{1,1}^{1} & \cdots & a_{1, T}^{1} \\
a_{2,1}^{1} & \cdots & a_{2, T}^{1} \\
\vdots & \ddots & \vdots \\
a_{M, 1}^{1} & \cdots & a_{M, T}^{1}
\end{array}\right], \boldsymbol{A}_{2}=\left[\begin{array}{ccc}
a_{1,1}^{2} & \cdots & a_{1, T}^{2} \\
a_{2,1}^{2} & \cdots & a_{2, T}^{2} \\
\vdots & \ddots & \vdots \\
a_{M, 1}^{2} & \cdots & a_{M, T}^{2}
\end{array}\right], \\
\cdots, \boldsymbol{A}_{Q}=\left[\begin{array}{ccc}
a_{1,1}^{Q} & \cdots & a_{1, T}^{Q} \\
a_{2,1}^{Q} & \cdots & a_{2, T}^{Q} \\
\vdots & \ddots & \vdots \\
a_{M, 1}^{Q} & \cdots & a_{M, T}^{Q}
\end{array}\right]
\end{gathered}
$$

is given by

$$
\begin{aligned}
& \boldsymbol{X}^{\mathrm{LDC}}[i] \\
& =s_{1}[i]\left[\begin{array}{ccc}
a_{1,1}^{1} & \cdots & a_{1, T}^{1} \\
a_{2,1}^{1} & \cdots & a_{2, T}^{1} \\
\vdots & \ddots & \vdots \\
a_{M, 1}^{1} & \cdots & a_{M, T}^{1}
\end{array}\right]+s_{2}[i]\left[\begin{array}{ccc}
a_{1,1}^{2} & \cdots & a_{1, T}^{2} \\
a_{2,1}^{2} & \cdots & a_{2, T}^{2} \\
\vdots & \ddots & \vdots \\
a_{M, 1}^{2} & \cdots & a_{M, T}^{2}
\end{array}\right]
\end{aligned}
$$

$$
+\ldots+s_{Q}[i]\left[\begin{array}{ccc}
a_{1,1}^{Q} & \cdots & a_{1, T}^{Q} \\
a_{2,1}^{Q} & \cdots & a_{2, T}^{Q} \\
\vdots & \ddots & \vdots \\
a_{M, 1}^{Q} & \cdots & a_{M, T}^{Q}
\end{array}\right]
$$

where the DMs are optimized using the power constraint of [5]:

$$
\operatorname{tr}\left(\boldsymbol{A}_{q} \boldsymbol{A}_{q}^{H}\right)=\frac{T}{Q} .
$$

By contrast, the STSK codeword $\boldsymbol{X}^{\text {STSK }}[i]$ transmitted during signaling interval $i$ is generated by activating a single $\mathrm{DM} \boldsymbol{A}_{q}[i]$ in conjunction with the $\mathcal{L}$-PSK/QAM symbol $s_{q}[i]$. Hence, a total of $\log _{2}(Q \cdot \mathcal{L})$ source bits are mapped to each space-time block $\boldsymbol{X}[i] \in \mathcal{C}^{M \times T}$ by the STSK scheme according to [8]

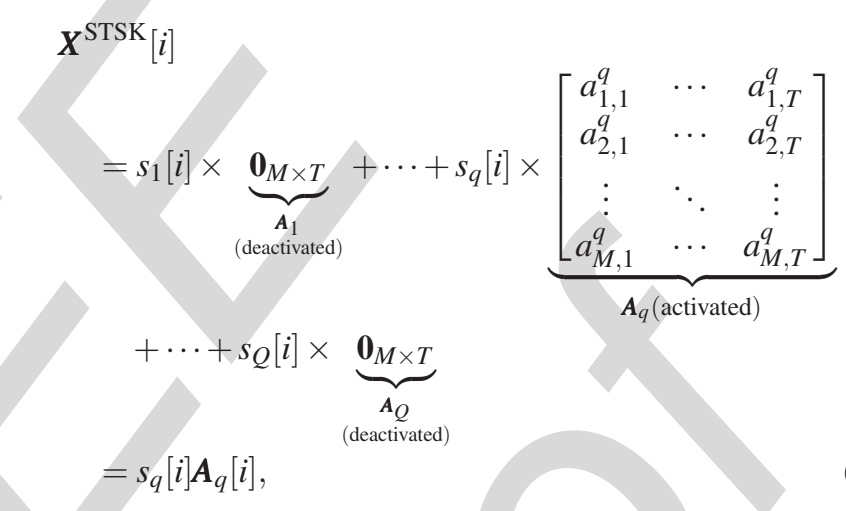

where $\mathbf{0}_{M \times T}$ is an $(M \times T)$-element zero matrix representing the deactivated DM and the $\mathcal{L}$-PSK/QAM symbol $s_{q}[i]$ is represented by $B_{1}=\log _{2} \mathcal{L}$ number of input bits, whereas the specific dispersion matrix $\boldsymbol{A}_{q}[i]$ is selected from the set of $Q \mathrm{DMs}$, $\boldsymbol{A}_{q},(q=1,2, \ldots, Q)$ by the $B_{2}=\log _{2} Q$ number of input bits. Additionally, in order to maintain a unity average transmission power within each STSK symbol duration, each of the DMs has to obey the power constraint of [8]:

$$
\operatorname{tr}\left(\boldsymbol{A}_{q} \boldsymbol{A}_{q}^{H}\right)=T
$$

where $\operatorname{tr}(\bullet)$ indicates the trace of the matrix " $\bullet$."

Since the LDC transmit codeword of (2) incorporates all the $Q$ DMs as well as all the $Q$ constellation symbols, LDCs impose a high decoding complexity [4], [5]. This is due to the presence of different $s_{q} \boldsymbol{A}_{q},(q=1,2, \ldots, Q)$ terms in (2) which introduce interference among themselves during the decoding stage and is referred to as the inter-stream interference [8], [10] in this treatise. The SM and the STSK systems activate a single antenna and a single DM, respectively, at each signaling interval. We refer to this as the single-stream transmission protocol, which does not impose any inter-stream interference [8], [10].

The benefits of the STSK scheme may be summarized as follows [8], [10]:

- The STSK scheme is capable of striking the same DMT as the LDC, but at a substantially reduced complexity. On the other hand, STSK is capable of achieving both transmit 
as well as receive diversity gains, while the SM and SSK schemes attain only receive diversity gain.

- Since only a single one of the $Q$ DMs is activated at any symbol instant, the STSK scheme imposes no interstream interference and - as a further benefit—a singlestream based ML detection can be employed [8].

- STSK is capable of adaptively supporting an arbitrary number of transmit as well as receive AEs.

The normalized throughput per symbol duration of the STSK scheme may be expressed as [8]

$$
R=\frac{\log _{2}(\mathcal{L} \cdot Q)}{T} \quad\left[\frac{\text { bit }}{\text { symbol duration }}\right] .
$$

Furthermore, the STSK system is uniquely and unambiguously specified by the parameters $(M, N, T, Q)$ in conjunction with the classic $\mathcal{L}$-PSK or $\mathcal{L}$-QAM modulation.

1) DM Optimization for STSK: The performance of the STSK system substantially depends on the choice of the DMs. More specifically, it depends firstly on the specific OF utilized and secondly on the DM optimization procedure. The optimization of the set of DMs is thus of utmost importance for the performance of the STSK scheme.

a) Random search based DM optimization: In the STSK encoder described by (4), $Q$ DMs, $\boldsymbol{A}_{q}(q=1, \ldots, Q)$ are preassigned to the transmitter in advance of transmission, which may be generated by searching exhaustively through a large set of randomly generated matrices and selecting the best ones using some optimization criterion as mentioned in [5] and detailed further in [38]. More specifically, the search process consists of generating a set of for example $10^{6} \mathrm{DMs}$, which have entries distributed according to the complex Gaussian distribution $\operatorname{e\mathcal {N}}(0,1)$ and which are normalized in order to satisfy the power-constraint criterion of (5) and then picking the best $Q$ matrices, $\boldsymbol{A}_{q}(q=1, \ldots, Q)$. More explicitly, the "quality" of the set generated is evaluated in terms of an OF, such as for example, the corresponding continuous input continuous output memoryless channel (CCMC) capacity [38], the discrete input continuous output memoryless channel (DCMC) capacity [71] and the pairwise error probability (PEP) [5] in terms of the rank and/or determinant criterion of [5], [26] etc.

b) Genetic algorithm aided DM optimization: In addition to the random search-based DM optimization mentioned above, genetic algorithm (GA) may also be employed for reducing the computational burden by searching through only a fraction of the DMs, yet finding the best ones with a high probability [59], [72]. The flowchart of the GA process for the optimization of the STSK spreading matrices is portrayed in Fig. 4. GAs [73] constitute adaptive heuristic search algorithms, where a population of individuals follows the evolutionary concepts of natural selection and genetic operators of crossover and mutation to optimize the OF or fitness function. In the DM optimization problem, an individual is defined as a set of DMs, which will be used for constructing the STSK scheme under the power constraint of (5). The initial population of DMs is randomly selected from a complex-valued Gaussian distribution of zero mean and unity variance. Thereafter the algorithm proceeds recursively. Each recursive stage is termed as a generation and the process is continued up to a pre-specified number of generations.

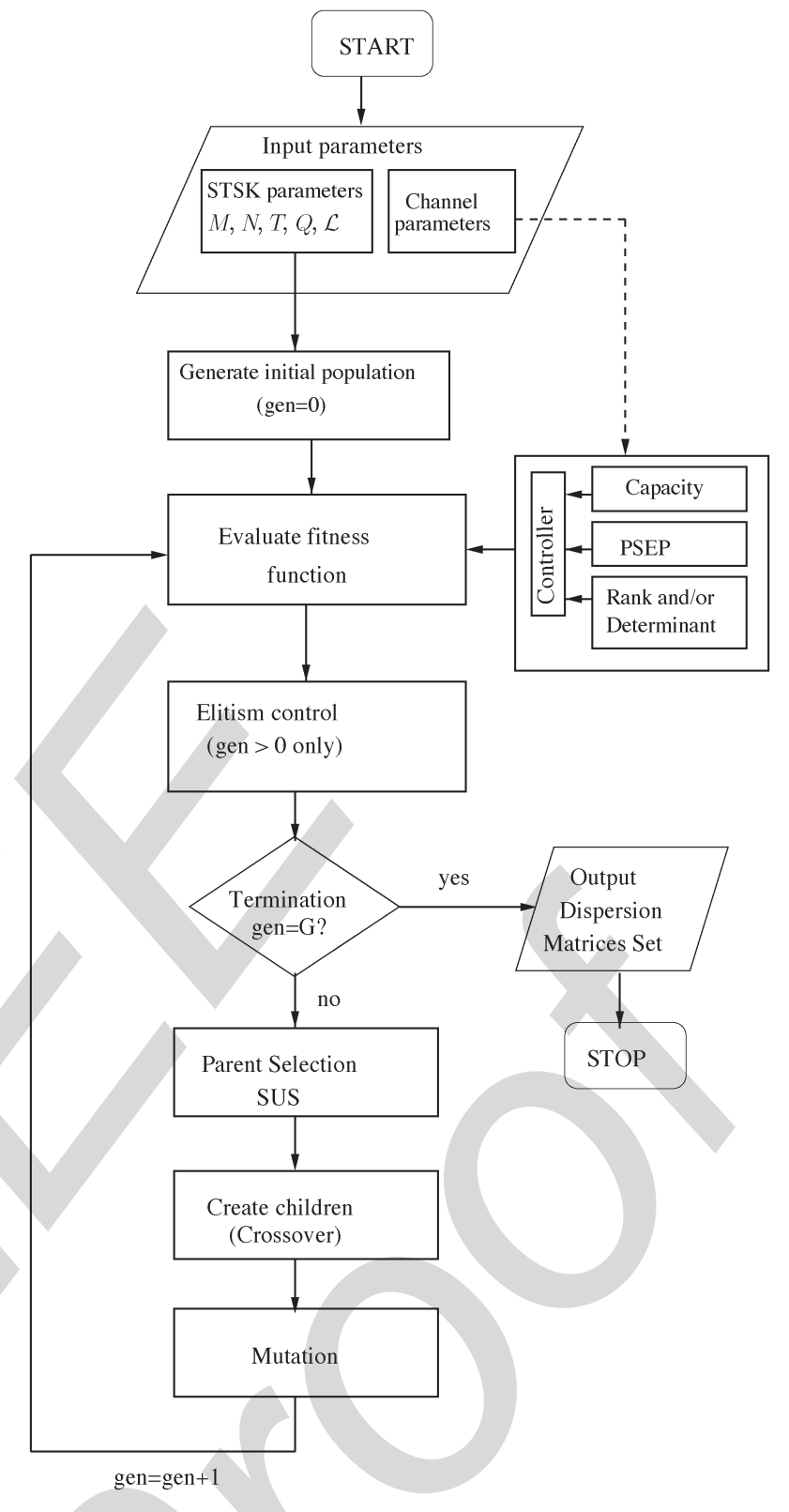

Fig. 4. Generation of the GA-optimized dispersion matrix set to be used in STSK.

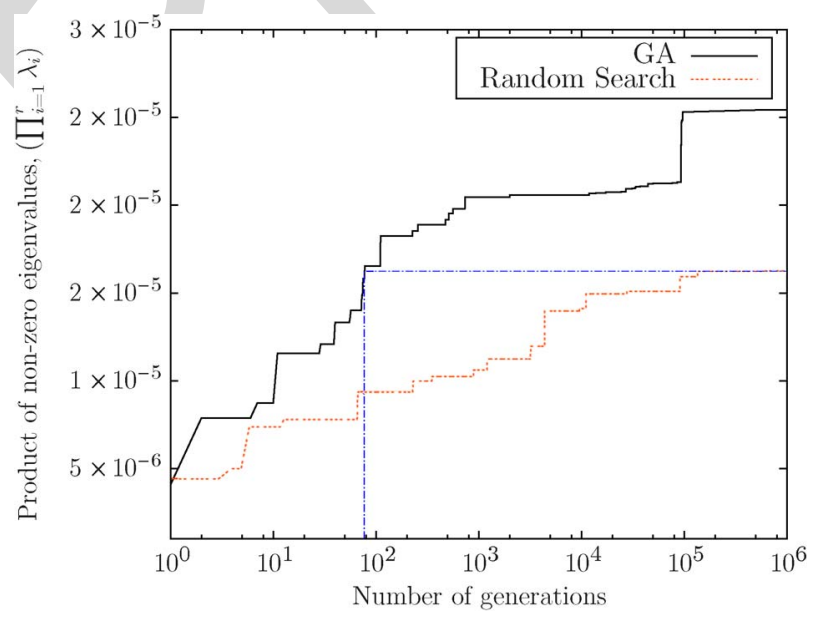

Fig. 5. Fitness function versus the number of generations in both GA optimization and in random search. 


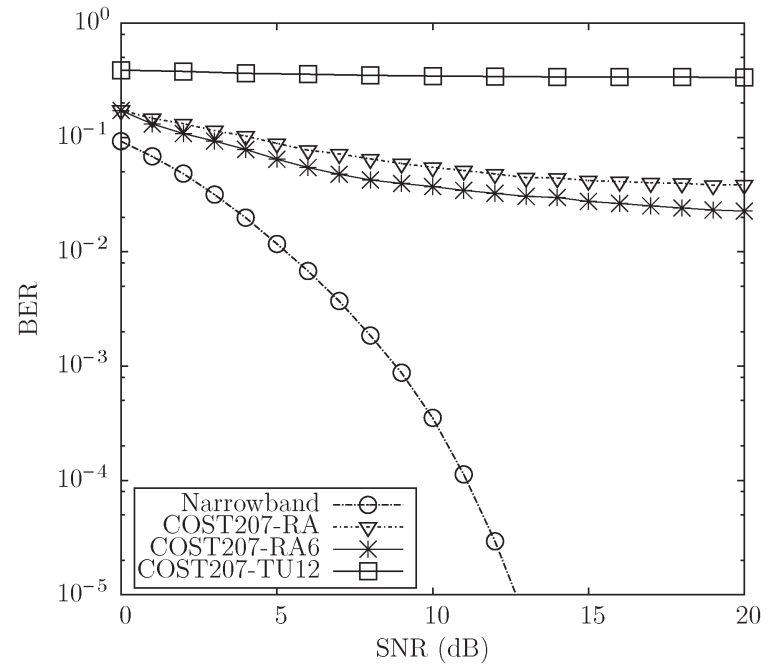

Fig. 6. BER performance of STSK $(2,2,2,2)$ scheme in conjunction with BPSK modulation using the optimal ML detector of [8] under different channel conditions. Although STSK performs well in non-dispersive channels, its performance in multipath channels becomes poor [12]. The system parameters are listed in Table III.

The performance of the GA-based DM optimization can be visualized in Fig. 5. Observe that for the fitness function as defined by the determinant criterion of [26], we approach the same fitness value at about 80 generations which were only approached by the random search after $10^{6}$ iterations. This clearly demonstrates the efficacy of the GA based optimization of DMs. Further insights on the optimization of the DMs for the STSK system may be found in [51], [60], [74].

2) Wideband Channels and the Performance of STSK in Different Channels: A time-invariant broadband channel has an impulse response, which can be modelled as [75], [76]:

$$
h(t, \tau)=\sum_{l=0}^{(L-1)} a_{l} g_{l}(t) \delta\left(\tau-\tau_{l}\right),
$$

where $L$ is the number of multipath components in the channel, $a_{l}, \tau_{l}$, and $g_{l}(t)$ are the channel's envelope, delay and Rayleighdistributed magnitude fading process exhibiting a particular normalized Doppler frequency $f_{d}$ respectively, associated with the $l$-th path, while $\delta($.$) is the Dirac Delta function. In fact,$ the wideband propagation channel is constituted by the superposition of a number of dispersive fading paths, suffering from various attenuations and delays, aggravated by the Doppler shift as a result of the mobile station (MS)'s movement.

The single-carrier STSK is characterized both in narrowband and wideband environments in Fig. 6. The basic simulation parameters are listed in Table III. It is demonstrated in Fig. 6 that the single-carrier STSK $(2,2,2,2)$ scheme operating in conjunction with BPSK modulation works well in narrowband scenarios, but it exhibits a severe error floor in dispersive channels, when the COST207-RA [77] channel model is considered. In the more dispersive typical urban (TU) scenario [75], [77], the STSK scheme suffers from a substantial further performance degradation. The simulation results characterizing STSK for different channel situations are shown in Fig. 6. Observe that the STSK scheme's bit-error ratio (BER) performance is better
TABLE III

Main System Parameters for STSK In DifFERENT ChanNels

\begin{tabular}{|l|r|}
\hline Simulation parameter & Values \\
\hline Fast fading model & 0.01 \\
Doppler frequency & Correlated Rayleigh fading \\
Channel specification & Narrowband channel \\
No. of OFDM subcarriers & 64 \\
Length of cyclic prefix & 32 \\
No. of Tx AE, $M$ & 2 \\
No. of Rx AE, $N$ & 2 \\
No. of Tx time slots, $T$ & 2 \\
No. of dispersion matrices & $Q=2,4$ \\
STSK specification & $\mathcal{L}=2,4$ \\
Modulation order & $(2,2,2, Q), Q=2,4$ \\
\hline
\end{tabular}

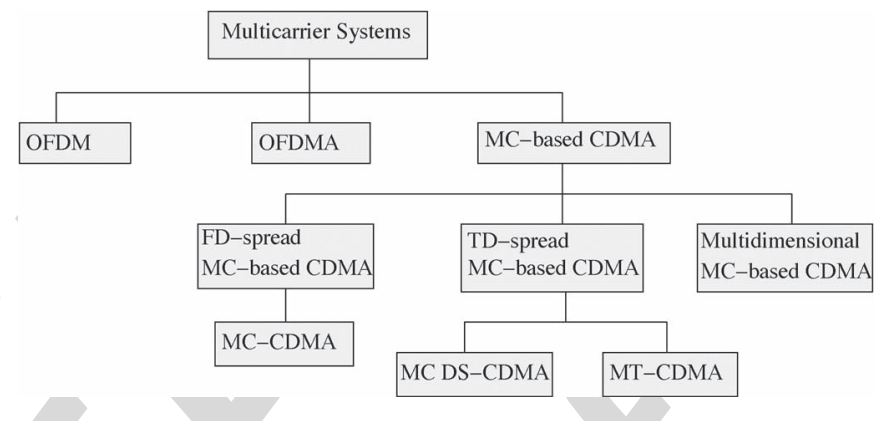

Fig. 7. Simplified family-tree of multicarrier systems.

for transmission over the COST207-RA6 channel model than that over the COST207-RA model, although the number of channel taps is higher in the COST207-RA6 model. This is because although the COST207-RA model has less taps, it has a higher total delay spread than the COST207-RA6 channel model.

The eroded performance of the single-carrier STSK scheme constituted the motivation for conceiving MC based STSK systems. A suite of MC systems, such as OFDM, MC-CDMA, OFDMA and/or SC-FDMA combined both with coherently and differentially detected STSK, was conceived. A range of coherent and noncoherent cooperative MC-CDMA STSK schemes employing SR for recovering the half-duplex throughput loss of conventional relaying schemes was also conceived. Let us now have a discourse on MC-aided STSK systems.

\section{MIMO-MC DESIGNS: EXAMPLE OF THE OFDM-AIDED STSK}

The simplified family-tree of MC systems is shown in Fig. 7. The most widespread family is constituted by OFDM [78]. The combination of OFDM with MIMO systems has attracted substantial interests due to OFDM's capability of converting realistic wideband channels into a number of low-rate, nondispersive subchannels [79]-[81]. Furthermore, OFDM facilitates the employment of single-tap FD equalizers at the receiver, which is highly beneficial in the context of MIMO systems.

\section{A. OFDM and MIMO-OFDM}

OFDM was ratified by numerous standardization bodies, such as the European Digital Audio Broadcasting (DAB) [82] 
TABLE IV

MAJOR CONTRIBUTIONS ON OFDM

\begin{tabular}{|c|c|c|}
\hline Year & Author(s) & Contribution \\
\hline 1966 & Chang [86] & The concept of OFDM was proposed for dispersive channels. \\
\hline 1970 & Chang [91] & U.S. Patent $3,488,445$ on OFDM was issued. \\
\hline 1971 & $\begin{array}{l}\text { Weinstein and } \\
\text { Ebert [87] }\end{array}$ & DFT was first applied for OFDM modulation. \\
\hline 1985 & Cimini $[88]$ & The feasibility of OFDM in mobile communications was studied. \\
\hline 1987 & $\begin{array}{l}\text { Alard and Lasalle } \\
\text { [89] }\end{array}$ & Proposed OFDM for digital broadcasting. \\
\hline 1991 & Cioffi [92] & Introduced the ANSI ADSL standard. \\
\hline 1999 & & IEEE 802.11a standard \\
\hline 2000 & $\begin{array}{lcr}\text { V. Nee and } \\
\text { Prasad }[93]\end{array}$ & First book on OFDM. \\
\hline 2003 & Hanzo et al. [94] & $\begin{array}{l}\text { Design-recipes and analytical insights were detailed in the context of broad- } \\
\text { band multiuser communications, WLANs and broadcasting. }\end{array}$ \\
\hline 2005 & Williams et al. [95] & A pre-FFT synchronisation method was designed for OFDM. \\
\hline 2009 & Chen et al. [96] & Spectrum sensing of pilot tone based OFDM systems was studied. \\
\hline 2014 & Sahin et al. [97] & $\begin{array}{l}\text { Multicarrier schemes are evaluated from the perspective of practical imple- } \\
\text { mentation aspects. }\end{array}$ \\
\hline
\end{tabular}

TABLE V

MAJOR CONTRIBUTIONS ON MIMO-OFDM

\begin{tabular}{|c|c|c|}
\hline Year & Author(s) & Contribution \\
\hline \multirow{2}{*}{2001} & Blum et al. $[90]$ & Improved space-time coding was proposed for MIMO-OFDM . \\
\hline & $\begin{array}{l}\text { Piechocki et } \\
\text { al. [98] }\end{array}$ & Studied the performance of ML decoding-aided V-BLAST OFDM. \\
\hline \multirow{3}{*}{2002} & Bolcskei et al. [99] & $\begin{array}{l}\text { Both the ergodic capacity and the outage capacity of spatial multiplexing aided } \\
\text { MIMO OFDM were determined. }\end{array}$ \\
\hline & Li et al. [100] & $\begin{array}{l}\text { Space-time coding based MIMO OFDM was proposed for mitigating the ISI } \\
\text { and for enhancing the system's capacity. }\end{array}$ \\
\hline & $\begin{array}{l}\text { Stamoulis } \\
\text { al. }[101]\end{array}$ & $\begin{array}{l}\text { Studied STBC based MIMO OFDM for transmission over time-varying } \\
\text { channels. }\end{array}$ \\
\hline \multirow[t]{2}{*}{2003} & $\begin{array}{l}\text { El-Gamal } \\
\text { al. }[102]\end{array}$ & $\begin{array}{l}\text { Employed OFDM for frequency-selective MIMO channels to construct space- } \\
\text { frequency codes (SFC) for exploiting the FD diversity. }\end{array}$ \\
\hline & Moon et al. [103] & $\begin{array}{l}\text { Proposed a peak-to-average power ratio (PAPR) control scheme for MIMO- } \\
\text { OFDM. }\end{array}$ \\
\hline \multirow[t]{2}{*}{2004} & Stuber et al. [79] & $\begin{array}{l}\text { Summarized the physical layer research challenges of MIMO-OFDM wireless } \\
\text { systems. }\end{array}$ \\
\hline & $\begin{array}{l}\text { Huang } \\
\text { Letaief [104] }\end{array}$ & $\begin{array}{l}\text { A coded OFDM-aided MIMO symbol-based space diversity scheme was } \\
\text { conceived. }\end{array}$ \\
\hline \multirow[t]{2}{*}{2005} & Su et al. $[105]$ & $\begin{array}{l}\text { Proposed a full-rate, full-diversity OFDM-aided space-frequency code for } \\
\text { MIMO assisted wireless systems. }\end{array}$ \\
\hline & $\begin{array}{l}\text { Borgmann and } \\
\text { Bolcskei [106] }\end{array}$ & $\begin{array}{l}\text { Non-coherent space-frequency coded OFDM was proposed for dispersive } \\
\text { MIMO channels. }\end{array}$ \\
\hline 2006 & $\begin{array}{ll}\text { Liew } & \text { and } \\
\text { Hanzo [107] } & \end{array}$ & $\begin{array}{l}\text { Investigated space-time block and space-time trellis codes versus adaptive } \\
\text { modulation and coding (AMC) aided OFDM. }\end{array}$ \\
\hline 2007 & $\begin{array}{l}\text { Jiang } \\
\text { Hanzo [40] }\end{array}$ & $\begin{array}{l}\text { Reduced-complexity genetic algorithm assisted multiuser MIMO-OFDM was } \\
\text { investigated. }\end{array}$ \\
\hline 2013 & $\begin{array}{l}\text { Miridakis and Ver- } \\
\text { gados [108] }\end{array}$ & $\begin{array}{l}\text { Reviewed successive interference cancellation schemes in the context of } \\
\text { single-antenna and multiple-antenna OFDM systems. }\end{array}$ \\
\hline 2014 & $\begin{array}{l}\text { Al-Dweik et } \\
\text { al. }[109]\end{array}$ & $\begin{array}{l}\text { A novel technique was proposed to enhance the robustness of conventional } \\
\text { MIMO-OFDM systems. }\end{array}$ \\
\hline
\end{tabular}

and the Digital Video Broadcasting (DVB) [83] standards and Local Area Mobile Wireless Networks, such as the IEEE 802.11a [84] and HIPERLAN/2 [85].

The major contributions on OFDM and MIMO-OFDM are summarized in Table IV and Table V, respectively. The first
OFDM scheme was proposed by Chang [86] in 1966 for dispersive channels. Weinstein et al. [87] demonstrated that OFDM modulation/demodulation can be efficiently performed using the DFT, which was a driving force behind the development of the OFDM scheme. In 1985, Cimini [88] proposed OFDM 


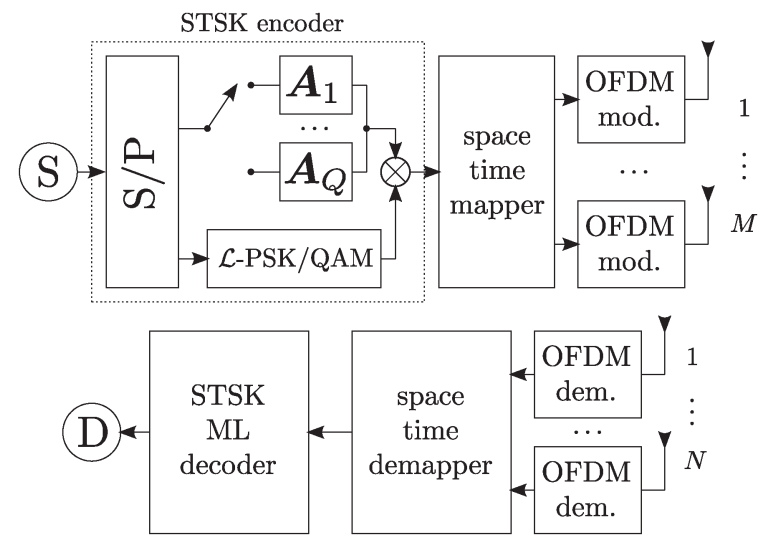

Fig. 8. The transceiver architecture of the OFDM-aided STSK system [11].

for mobile communications, whereas its application for digital broadcasting was proposed by Alard and Lassale [89] in 1985 and 1987, respectively.

In 2001, Blum et al. [90] proposed an improved space-time coding arrangement for MIMO-OFDM wireless systems. As a further advance, Stuber et al. [79] summarized the physical layer research challenges of MIMO-OFDM.

In the spirit of the above-mentioned MIMO-OFDM schemes, a range of MC STSK systems were developed for either single-user or multiuser communications over dispersive channels [11]-[14]. Before proceeding to the multiuser MC STSK schemes, let us first focus on OFDM-aided STSK scheme which was conceived for single-user systems. In [54], the authors proposed space-time-frequency shift keying (STFSK) as a technique capable of exploiting space-, time- and frequency diversity. Nevertheless, this realization of the STFSK scheme suffers from the typical drawbacks of non-coherent FSK demodulation. These impediments of STFSK may be eliminated by reduced-rate OFDM-style parallel transmissions over numerous non-dispersive sub-carriers.

\section{B. OFDM-Aided STSK}

OFDM-aided STSK [11] was conceived for mitigating the performance erosion of STSK schemes operating in wideband channels as demonstrated in Fig. 6 of Section II-C2. The basic idea of the OFDM-aided STSK system is to use the OFDM technique in order to create a number of parallel FD subchannels, where the sub-channel bitrate is sufficiently low for the sub-channel symbols to avoid dispersion. The model of [79] has been adopted for realizing an STSK-based MIMO-OFDM system obeying the system's architecture of Fig. 8. Having generated the STSK blocks, the blocks of symbols are then mapped to $N_{\mathrm{c}}$ subcarriers. As shown in Fig. 9, $N_{\mathrm{c}}$ STSK codewords are arranged in parallel, which constitute an OFDM-STSK frame. Furthermore, OFDM modulation is carried out over the shaded $N_{c}$-length symbol-pipe at AE $m(m=1, \ldots, M)$ during time slot $T_{i}\left(T_{i}=1, \ldots, T\right)$, which represents an OFDM symbol. The time-domain (TD) STSK-OFDM signal transmitted from the $m$ th transmit $\mathrm{AE}$ at time slot $T_{i}$ may be formulated as [94], [110], [111]:
Transmit AE index, $m$

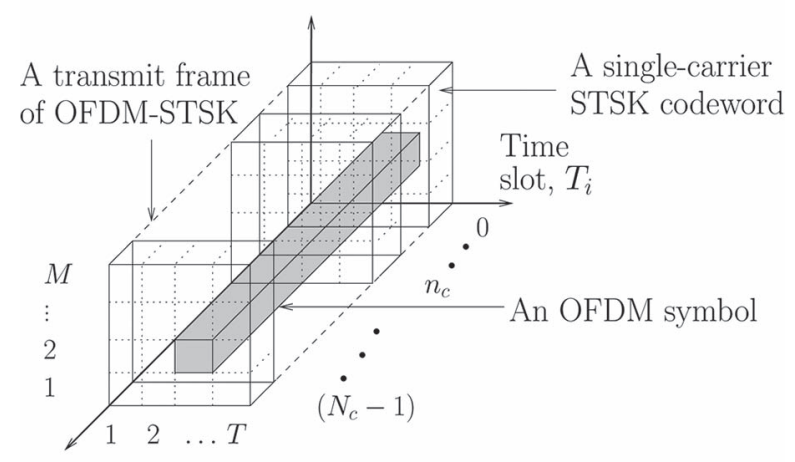

Subcarrier, $n_{c}$

Fig. 9. Formation of an STSK-OFDM frame consisting of $N_{c}$ parallel STSK blocks to be transmitted over $N_{c}$ parallel subcarriers. The shaded symbol-pipe shown represents one of the $(M \cdot T)$ OFDM symbols [69].

$$
x_{m, T_{i}}(t)=\frac{1}{\sqrt{N_{c}}} \sum_{n_{c}=0}^{\left(N_{c}-1\right)} X_{m, T_{i}}\left[n_{c}\right] e^{j 2 \pi f_{n_{c}} t}, \quad 0 \leq t \leq N_{c} \mathcal{T}
$$

where $X_{m, T_{i}}\left[n_{c}\right]$ is the $n_{c}$-th data symbol, while $N_{c} \mathcal{T}$ is the length of the OFDM symbol. In order to maintain the orthogonality of the subcarriers, the subcarrier frequencies have to be equally spaced by $f_{n_{c}}=\frac{n_{c}}{N_{\mathcal{C}} \mathcal{T}}$ and the TD sampling of the OFDM symbol can be formulated as:

$$
\begin{aligned}
x_{m, T_{i}}\left(n_{s} \mathcal{T}\right)=\frac{1}{\sqrt{N_{c}}} \sum_{n_{c}=0}^{\left(N_{c}-1\right)} X_{m, T_{i}}\left[n_{c}\right] e^{j 2 \pi \frac{n_{s} n_{c}}{N_{c}}}, \\
0 \leq n_{s} \leq\left(N_{c}-1\right)
\end{aligned}
$$

which is expressed in terms of the $N_{c}$-point IDFT of the symbol stream $\boldsymbol{X}_{m, T_{i}}=\left\{X_{m, T_{i}}\left[n_{c}\right]\right\}_{n_{c}=0}^{\left(N_{c}-1\right)}$ as:

$$
\boldsymbol{x}_{m, T_{i}}=\operatorname{IDFT}_{N_{c}}\left\{\boldsymbol{X}_{m, T_{i}}\right\}
$$

where $m=1, \ldots, M$ and $T_{i}=1, \ldots, T$. The usual CP, which has to be longer than the channel's delay spread, is appended to each of the TD OFDM symbols in order to eliminate the effects of ISI.

Let the signals from the transmit AEs be transmitted over a frequency-selective channel, where the discrete-time CIR may be modeled as:

$$
\boldsymbol{H}\left[n_{c}\right]=\sum_{l=0}^{L-1} \boldsymbol{H}[l] \delta\left[n_{c}-l\right] \in \mathbb{C}^{N \times M},
$$

where the matrix $\boldsymbol{H}[l] \in \mathbb{C}^{N \times M}$ has complex-valued normally distributed entries according to $\mathcal{E N}\left(\mu_{l}, \sigma_{l}^{2}\right)$ for every channel path $l=0, \ldots,(L-1)$ and $\delta[\cdot]$ is the discrete-time Dirac delta function. After applying the $N_{c}$-point DFT operation at each receive $\mathrm{AE}$ and defining the FD channel transfer matrix $\tilde{\boldsymbol{H}}_{n, m}=\operatorname{diag}\left\{\tilde{h}_{n, m}[0], \tilde{h}_{n, m}[1], \ldots, \tilde{h}_{n, m}\left[N_{c}-1\right]\right\} \in \mathbb{C}^{N_{c} \times N_{c}}$ as a diagonal matrix [13], [94] whose diagonal elements are $\tilde{\boldsymbol{h}}_{n, m}=$ $\operatorname{DFT}_{N_{c}}\left\{\boldsymbol{h}_{n, m}\right\}$, the FD channel-output matrix $\boldsymbol{Y}$ at the receiver may be expressed by 


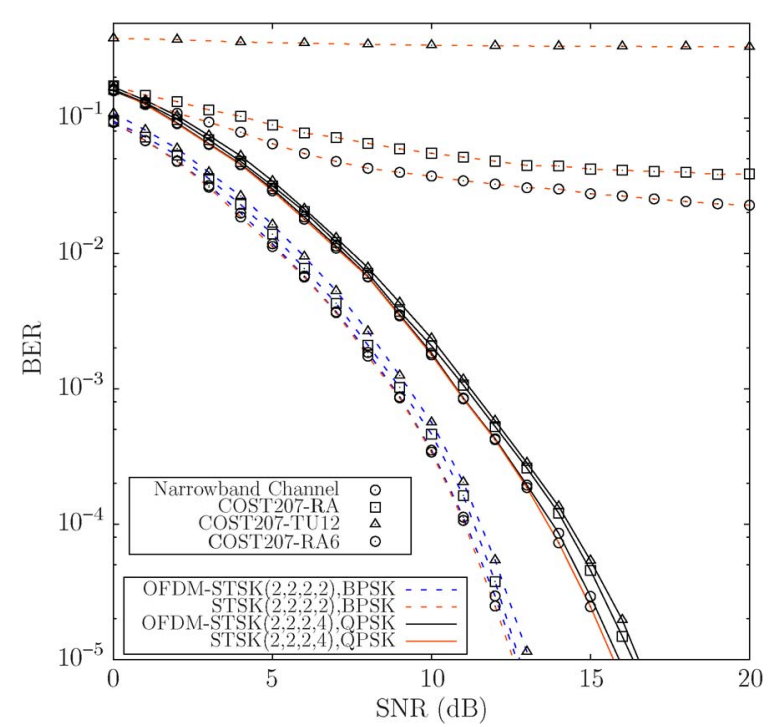

Fig. 10. Performance of the OFDM-aided STSK system of Fig. 8 in dispersive channels. No channel-coding was used and the optimal ML detector of [8] was employed. OFDM-aided STSK exhibits a performance in dispersive channels, which is similar to that in nondispersive environments.

$$
\boldsymbol{Y}\left[n_{c}\right]=\tilde{\boldsymbol{H}}\left[n_{c}\right] \boldsymbol{X}\left[n_{c}\right]+\boldsymbol{V}\left[n_{c}\right], \quad n_{c}=0, \ldots,\left(N_{c}-1\right)
$$

where $\boldsymbol{Y}\left[n_{c}\right] \in \mathbb{C}^{N \times T}, \tilde{\boldsymbol{H}}\left[n_{c}\right] \in \mathbb{C}^{N \times M}, \boldsymbol{X}\left[n_{c}\right] \in \mathbb{C}^{M \times T}$, and $\boldsymbol{V}\left[n_{c}\right] \in$ $\mathbb{C}^{N \times T}$. Following the OFDM demodulation at the space-time demapper, the received symbols $\boldsymbol{Y}\left[n_{c}\right] \in \mathbb{C}^{N \times T}$ are then passed through the single-stream ML decoder [8]. The singlestream based ML estimates $\left(\hat{l}_{c}, \hat{q}\right)$ of the $n_{c}$-th STSK-OFDM symbol and its constituent transmitted bits are obtained from [7], [8] as:

$$
\left(\hat{l}_{c}\left[n_{c}\right], \hat{q}\left[n_{c}\right]\right)=\underset{l_{c}, q}{\arg \min }\left\{\left\|\overline{\boldsymbol{Y}}\left[n_{c}\right]-\overline{\tilde{\boldsymbol{H}}}\left[n_{c}\right] \boldsymbol{\chi} \boldsymbol{K}_{l_{c}, q}\right\|^{2}\right\}
$$

where

$$
\begin{aligned}
\overline{\boldsymbol{Y}}\left[n_{c}\right] & =\operatorname{vec}\left(\boldsymbol{Y}\left[n_{c}\right]\right) \in \mathbb{C}^{N T \times 1}, \\
\overline{\tilde{\boldsymbol{H}}}\left[n_{c}\right] & =\boldsymbol{I}_{T} \otimes \tilde{\boldsymbol{H}}\left[n_{c}\right] \in \mathbb{C}^{N T \times M T}, \\
\boldsymbol{\chi} & =\left[\operatorname{vec}\left(\boldsymbol{A}_{1}\right), \cdots, \operatorname{vec}\left(\boldsymbol{A}_{Q}\right)\right] \in \mathbb{C}^{M T \times Q},
\end{aligned}
$$

and

$$
\boldsymbol{K}_{l_{c}, q}=[\underbrace{0, \cdots, 0}_{q-1}, s_{l_{c}}, \underbrace{0, \cdots, 0}_{Q-q}]^{T} \in \mathbb{C}^{Q \times 1} .
$$

The performance degradation of the single-carrier STSK system in wideband channels was quantified in Section II-C, which was substantially mitigated by the OFDM-aided STSK system of Fig. 8, as portrayed in Fig. 10. Observe from Fig. 10 that the OFDM-aided STSK performance in wideband channels essentially remains unaffected by the channel's dispersion. We see from Fig. 10 that if the channel is non-dispersive, there is virtually no difference in the performance of STSK, regardless whether OFDM has been incorporated into the system or not. In such channel conditions, both the STSK and the OFDM- aided STSK are capable of attaining excellent diversity versus multiplexing gain tradeoffs. By contrast, under dispersive channel conditions, OFDM-aided STSK is capable of maintaining its superiority not only in the rural scenario of the COST207RA channel model, but also in the typical urban scenario modeled by the COST207-TU12 channel model. In fact, the OFDM-aided STSK performance recorded in different channel situations remains more or less similar to that of the narrowband benchmarker.

\section{MC STSK SCHEMES FOR DISPERSIVE MULTIUSER SCENARIOS}

In this section, we provide a brief review of MC-based CDMA, OFDMA and SC-FDMA systems, which is followed by an overview of the amalgamated MC-CDMA aided STSK [12], [112] and OFDMA-assisted and SC-FDMA-aided STSK schemes for supporting multiuser transmissions over dispersive wireless channels. A reduced-complexity detector designed for the OFDMA/SC-FDMA-aided STSK scheme is also discussed.

\section{A. MC-Based CDMA, OFDMA, and SC-FDMA Systems}

The major contributions on MC-CDMA, SC-FDMA and MIMO SC-FDMA are summarized in Table VI. Historically speaking, in 1993, Fettweis et al. developed the MC-CDMA scheme of [113]. Chouly et al. [114] as well as DaSilva and Sousa developed the MC DS-CDMA scheme of [115], whereas the so-called multitone (MT)-CDMA scheme was developed by Vandendorpe [116], all in the same year. In 1997, Hara and Prasad [117] provided a well-cited overview of MC based CDMA systems.

MC-CDMA is a multiple access scheme, where the symbols of different users are first spread across the FD using unique, user-specific spreading sequences, which are then mapped to multiple parallel subcarriers [94], [117]. In the MC DS-CDMA scheme, the data stream is first divided into a number of parallel substreams, each substream is then spread across the TD with the aid of the spreading sequences and then transmitted over one of the subcarriers [117], [118]. The MT-CDMA scheme [116] relies on similar operations to those of MC DS-CDMA, except that the subcarriers are orthogonal to each other before spreading, but the spectrum of subcarriers no longer exhibits orthogonality after TD-spreading. There have also been substantial developments in the context of multidimensional spreading assisted MC-based CDMA schemes [119]-[121], which spread the user information across both the spatial-as well as time-domains or even over the spatial domain (SD). OFDMA [122] is a multiple access version of OFDM, where a block of subcarriers is allocated to the different users in a frequency-division multiple access fashion.

In the aftermath of widespread MC applications, singlecarrier modulation (SCM) is enjoying a renaissance, especially in the context of UL scenarios [123]. Traditionally equalization has been performed in the TD for single carrier transmissions. However, if the ISI spreads over more than for example 30-50 symbols, the signal processing complexity associated with this TD approach might become excessive [123]. As an alternative, FD equalization (FDE) was first studied in [124]. 
TABLE VI

MAJOR CONTRIBUTIONS ON MC-CDMA AND SC-FDMA

\begin{tabular}{|c|c|c|}
\hline Year & Author(s) & Contribution \\
\hline 1973 & $\begin{array}{l}\text { Walzman and Schwartz } \\
{[124]}\end{array}$ & Introduced frequency domain equalization (FDE). \\
\hline \multirow{4}{*}{1993} & Yee et al. [113] & $\begin{array}{l}\text { The concept of FD MC-CDMA combining FD-spreading and OFDM was } \\
\text { introduced. }\end{array}$ \\
\hline & Chouly et al. [114] & Combined multicarrier techniques to DS-CDMA. \\
\hline & DaSilva \& Sousa [115] & Developed MC DS-CDMA. \\
\hline & Vandendorpe [116] & Introduced the concept of multitone DS-CDMA. \\
\hline 1995 & Sari et al. [125] & Proposed SC-FDE for digital terrestrial TV broadcasting. \\
\hline 1997 & Hara and Prasad [117] & Provided an overview of MC-CDMA systems. \\
\hline 1998 & Sorger et al. [130] & $\begin{array}{l}\text { Introduced IFDMA applicable for both the uplink and the downlink of a } \\
\text { mobile radio communications system. }\end{array}$ \\
\hline \multirow{2}{*}{2002} & Falconer et al. [126] & SC-FDE was reviewed and compared to OFDM. \\
\hline & $\begin{array}{l}\text { Benvenuto and } \\
\text { Tomasin [131] }\end{array}$ & Proposed a new FD decision feedback equalizer (DFE) for SC-FDE. \\
\hline 2003 & Hanzo et al. [94] & $\begin{array}{l}\text { The state-of-the-art in OFDM and MC-CDMA aided broadband multiuser } \\
\text { communications, WLANs and broadcasting was reviewed. }\end{array}$ \\
\hline 2006 & Myung et al. [128] & Introduced the concept of SC-FDMA for the wireless uplink. \\
\hline 2007 & Wilzeck et al. [132] & $\begin{array}{l}\text { Conceived a MIMO-SC-FDMA system model and analyzed the effect of } \\
\text { carrier frequency offsets. }\end{array}$ \\
\hline 2008 & Berardinelli et al. [122] & $\begin{array}{l}\text { A comparative performance study of OFDMA and SC-FDMA was carried } \\
\text { out. }\end{array}$ \\
\hline \multirow{2}{*}{2010} & Ciochina \& Sari [133] & Another comparative study of OFDMA versus SC-FDMA was offered. \\
\hline & Benvenuto et al. [123] & $\begin{array}{l}\text { Provided a comprehensive review of nonlinear FDE in the context of single- } \\
\text { carrier modulation. }\end{array}$ \\
\hline 2012 & Dalakas et al. [134] & OFDMA and SC-FDMA were compared for satellite uplink. \\
\hline
\end{tabular}

However, the full benefit of the SC-FDE was gradually realized after Sari et al. [125] proposed it as a low-complexity solution to digital terrestrial TV broadcasting, which was reviewed by Falconer et al. [126], [127]. The concept of SC-FDMA as a DFTprecoded OFDMA arrangement was proposed as a multipleaccess scheme for the LTE system by Myung et al. [128] as well as by the Pan-European Wireless INitiative NEw Radio (WINNER) project [129] in the context of the uplink of wide area cellular systems. A comprehensive review of nonlinear FDE in the context of single-carrier modulation was provided in [123].

\section{B. MC-CDMA Aided STSK}

MC-CDMA-aided STSK was conceived in [12] for mitigating the performance erosion of the classic STSK scheme in dispersive channels, while supporting multiple users. The codewords generated by the STSK scheme are appropriately spread across the frequency-domain (FD) and transmitted over a number of parallel frequency-flat subchannels.

1) Transmission Protocol: Let us consider an MC-CDMA aided STSK system having $M$ transmit and $N$ receive AEs and communicating over frequency-selective Rayleigh fading channels, whose transmitter architecture is shown in Fig. 11. Furthermore, $N_{c}$ subcarriers are employed by the MC-CDMA modem for transmitting $N_{p}$ STSK codewords. In general, $N_{c}$ is related to $N_{p}$ by: $N_{c}=\left(N_{p} \times S_{f}\right)$. However, it is possible to spread each of the codewords across all the subcarriers, where $S_{f}=N_{c}$ [94]. As seen in Fig. 11, STSK codewords $\boldsymbol{X}^{u}$ are generated from the users' source information, which are further spread across the FD and are then mapped to a number of sub-

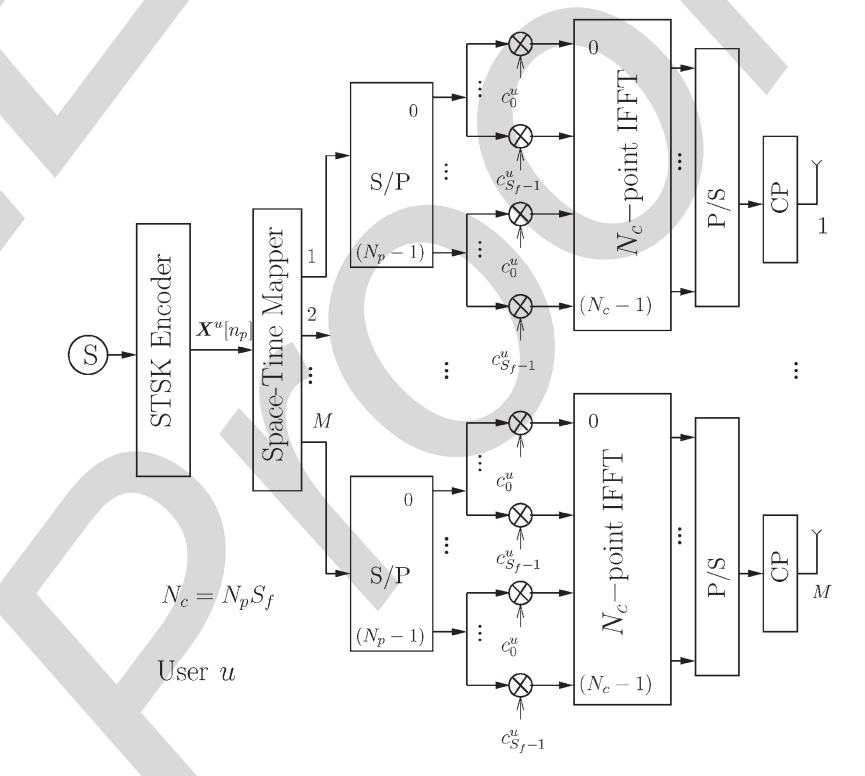

Fig. 11. Transmission model of the MC-CDMA aided multiuser STSK scheme of [12] corresponding to user $u$. Following space-time mapping, each of the TA symbols is spread across the FD before being mapped to the subcarriers.

carriers, before being transmitted using $M$ transmit AEs over $T$ time slots. A number of $N_{p}$ space-time codewords generated by the STSK encoder are spread across all the $N_{c}$ subcarriers by the user-specific spreading sequence, $\boldsymbol{C}^{u}=\left[c_{0}^{u}, c_{1}^{u}, \ldots, c_{S_{f}-1}^{u}\right]$, where $S_{f}$ represents the spreading factor and $u$ is the user index. To be more specific, assuming the relationship of $N_{c}=$ $\left(N_{p} \times S_{f}\right)$, FD spreading of the $N_{p}$ codeword symbols results in $N_{c}$ number of FD symbols of the spread codewords denoted 


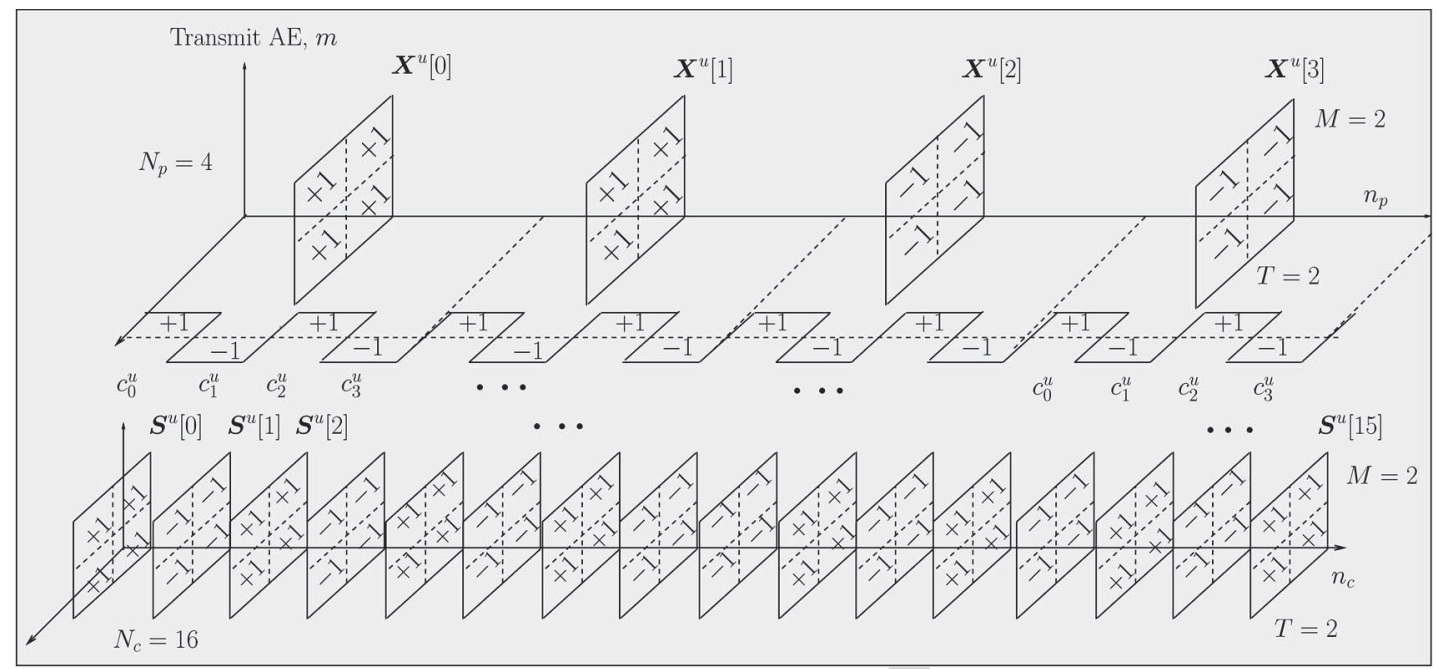

Fig. 12. FD spreading example of the STSK codewords in MC-CDMA-aided STSK system. 4 STSK codewords $\boldsymbol{X}^{u}[0], \boldsymbol{X}^{u}[1], \boldsymbol{X}^{u}[2]$, and $\boldsymbol{X}^{u}[3]$ are considered, which are spread in FD by the user-specific spreading sequence $\boldsymbol{C}^{u}=[+1,-1,+1,-1]$, resulting in 16 spread space-time matrices, which are denoted by $\boldsymbol{S}^{u}[0]$, $S^{u}[1], \ldots, S^{u}[15]$, respectively.

by $\boldsymbol{S}^{u}$. After the IDFT operation of Fig. 11, CP of appropriate length are incorporated for the sake of eliminating the effects of ISI. The mapping of the STSK codewords to the $N_{c}$ subcarriers is illustrated by the following example.

Example 1: FD spreading and STSK symbol mapping to MC-CDMA subcarriers: $N_{c}=16, S_{f}=4$

For the sake of exemplifying the FD spreading operation and the subsequent mapping of the spread codeword symbols to the orthogonal subcarriers, consider an MCCDMA aided STSK scheme employing $N_{c}=16$ subcarriers and a FD-spreading factor $S_{f}=4$. We will then take $N_{p}=N_{c} / S_{f}=16 / 4=4$ STSK codewords of a particular user $u$ and place them in parallel, as shown in Fig. 12. Let the 4 codewords be denoted by:

$$
\left[\boldsymbol{X}^{u}[0], \boldsymbol{X}^{u}[1], \boldsymbol{X}^{u}[2], \boldsymbol{X}^{u}[3]\right] .
$$

Let us further assume for example that the spreading sequence corresponding to user $u$ is:

$$
\boldsymbol{C}^{u}=[+1,-1,+1,-1] .
$$

Each of the codewords $\boldsymbol{X}^{u}$ will now be multiplied by each single chip of the spreading sequence $\boldsymbol{C}^{u}$, resulting in $4 \times 4=16$ spread codewords. More explicitly, $\boldsymbol{X}^{u}[0]$ multiplied by the chips will produce 4 spread codewords denoted by:

$$
\begin{aligned}
\boldsymbol{S}^{u}[0] & =c_{0}^{u} \boldsymbol{X}^{u}[0]=+1 \times \boldsymbol{X}^{u}[0], \\
\boldsymbol{S}^{u}[1] & =c_{1}^{u} \boldsymbol{X}^{u}[0]=-1 \times \boldsymbol{X}^{u}[0], \\
\boldsymbol{S}^{u}[2] & =c_{2}^{u} \boldsymbol{X}^{u}[0]=+1 \times \boldsymbol{X}^{u}[0], \\
\text { and } \quad \boldsymbol{S}^{u}[3] & =c_{3}^{u} \boldsymbol{X}^{u}[0]=-1 \times \boldsymbol{X}^{u}[0] .
\end{aligned}
$$

Thus the 4 codewords $\left[\boldsymbol{X}^{u}[0], \boldsymbol{X}^{u}[1], \boldsymbol{X}^{u}[2], \boldsymbol{X}^{u}[3]\right]$ will produce a total of 16 spread codewords denoted by:

$$
\left[\boldsymbol{S}^{u}[0], \boldsymbol{S}^{u}[1], \ldots, \boldsymbol{S}^{u}[15]\right] .
$$

In Fig. 12, we assume all the STSK codewords to be $(2 \times$ 2) - element matrices, i.e., we assume $M=2, T=2$. We also consider, for the sake of simplicity, all the elements of the STSK codewords $\boldsymbol{X}^{u}[0]$ and $\boldsymbol{X}^{u}[1]$ to be +1 , while those of $\boldsymbol{X}^{u}[2]$, and $\boldsymbol{X}^{u}[3]$ to be -1 . As a result, all the elements of the spread codewords $S^{u}[0], S^{u}[1], S^{u}[2]$, and $\boldsymbol{S}^{u}[3]$ are $+1,-1,+1$ and -1 , respectively. By contrast, all the elements of for example $S^{u}[8], S^{u}[9], S^{u}[10]$, and $S^{u}[11]$ are $-1,+1,-1$ and +1 , respectively.

The resultant 16 spread codeword symbols will then be mapped to the $N_{c}=16$ subcarriers using the IDFT operation.

2) Receiver: The signal received after transmission over a dispersive channel is demodulated by Fourier-transforming it after removal of CP. After CP removal and $N_{c}$-point DFT, the FD signal received is given by:

$$
\boldsymbol{Y}\left[n_{c}\right]=\underbrace{\tilde{\boldsymbol{H}}^{v}\left[n_{c}\right] \boldsymbol{S}^{v}\left[n_{c}\right]}_{\text {desired signal }}+\underbrace{\sum_{\substack{u=1 \\ u \neq v}}^{U} \tilde{\boldsymbol{H}}^{u}\left[n_{c}\right] \boldsymbol{S}^{u}\left[n_{c}\right]}_{\text {MUI }}+\underbrace{\boldsymbol{V}\left[n_{c}\right]}_{\text {noise }},
$$

where each element of the FD channel transfer matrix $\tilde{\boldsymbol{H}}^{u}\left[n_{c}\right] \in \mathbb{C}^{N \times M}$ is a diagonal matrix given by $\tilde{\boldsymbol{H}}_{n, m}^{u}=$ $\operatorname{diag}\left\{\tilde{h}_{n, m}^{u}[1], \tilde{h}_{n, m}^{u}[2], \ldots, \tilde{h}_{n, m}^{u}\left[N_{c}\right]\right\} \in \mathbb{C}^{N_{c} \times N_{c}}$, whose diagonal elements represent the FD channel transfer function between the $m$-th transmit and the $n$-th receive AE. Both singleuser detectors (SUDs) and multiuser detectors (MUDs) may be employed for the detection of the different users' signals [117]. Since the orthogonality amongst the different users' signals is destroyed by dispersive channels, the MUI cannot be eliminated if a low-complexity SUD is employed. The MUI degrades the resultant BER performance both in the downlink (DL) and the uplink (UL) multiuser scenarios. By contrast, the MUD jointly detects the information of all the users and hence an improved BER performance may be attained, albeit at the cost of imposing an increased decoding complexity. The source information of users in a particular space-time block indexed by $n_{p}$ is spread 


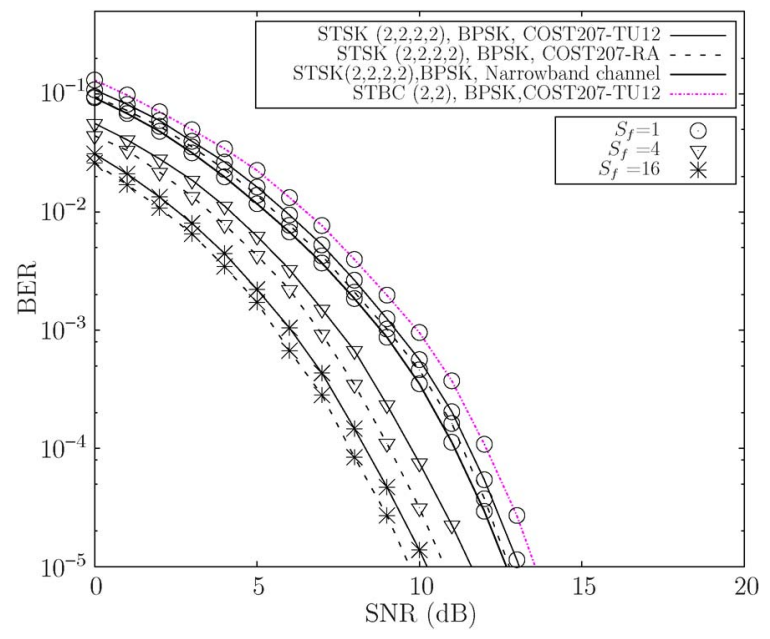

Fig. 13. Performance of single-user uncoded MC-CDMA STSK system of Fig. 11 both in narrowband and in dispersive channels. The performance of the MC-CDMA aided $\mathcal{G}_{2}-\operatorname{STBC}(M, N)=(2,2)$, BPSK and $\mathcal{G}_{2}-\operatorname{STBC}(2,2)$, QPSK is also shown as benchmarker.

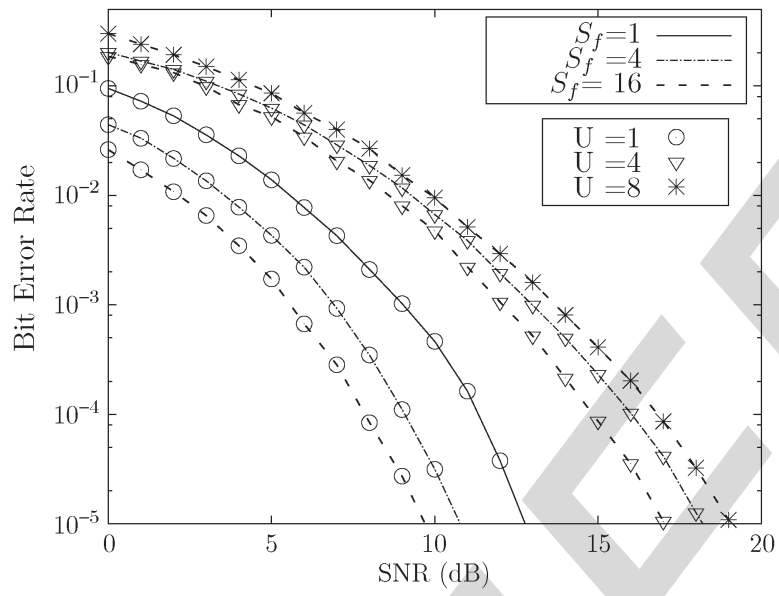

Fig. 14. BER performance of MC-CDMA aided STSK $(2,2,2,2)$, BPSK for the DL COST207-RA channel using different $S_{f}$ supporting $U$ users [12].

over $S_{f}$ number of outputs from $\overline{\boldsymbol{Y}}\left[n_{p} S_{f}\right]$ to $\overline{\boldsymbol{Y}}\left[\left(n_{p}+1\right) S_{f}-1\right]$. The single-user detector combines the signal energy spread over the different subcarriers employing a combining method, such as orthogonality-restoring combining (ORC) [117], EGC [22], [117] or MRC [22], [117]. Using MRC, the decision variables of the desired user $v$ may be obtained as [117]:

$$
\boldsymbol{z}^{v}\left[n_{p}\right]=\sum_{s_{f}=0}^{\left(S_{f}-1\right)} \boldsymbol{g}^{v}\left[n_{p} S_{f}+s_{f}\right] \boldsymbol{Y}\left[n_{p} S_{f}+s_{f}\right],
$$

where $\boldsymbol{g}^{v}\left[n_{p} S_{f}+s_{f}\right]$ is given by $\boldsymbol{g}^{v}\left[n_{p} S_{f}+s_{f}\right]=c_{s_{f}}^{v}\left[\tilde{\boldsymbol{H}}^{v}\left[n_{p} S_{f}+s_{f}\right]\right]^{H}$. Hence, the source bits of user $v$ may be estimated as [8], [13]

$$
\left(q^{v}\left[n_{p}\right], l_{c}^{v}\left[n_{p}\right]\right)=\underset{q^{v}, l_{c}^{v}}{\arg \min }\left\{\left\|\overline{\boldsymbol{z}}^{v}\left[n_{p}\right]-\boldsymbol{\chi} \boldsymbol{K}_{q^{v}, l_{c}^{v}}^{v}\right\|^{2}\right\} .
$$

A new single-stream maximum-likelihood (ML) MUD (ML-MUD) may also be employed [12] for the joint detection of the user signals, which amalgamates both the single-stream ML detector of [8], [10] and the MUD of [117].

The performances achievable by the MC-CDMA-aided STSK under both a single-user and a multiuser scenario are illustrated

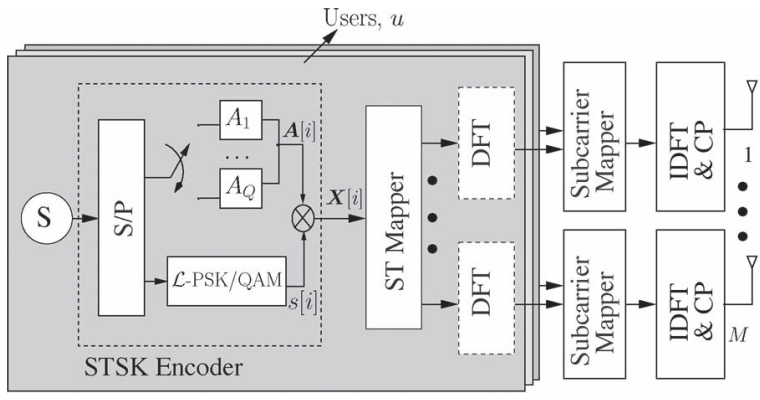

Fig. 15. Transmission model of the SC-FDMA aided STSK scheme. In OFDMA the dotted "DFT $N_{d}$ " block of the transmitter does not exist. The STSK mapper selects one out of the Q dispersion matrices along with one constellation symbol and the resultant space-time codewords are passed in different time slots through the OFDMA or SC-FDMA based multi-user transmission system, before being transmitted by the transmit AEs.

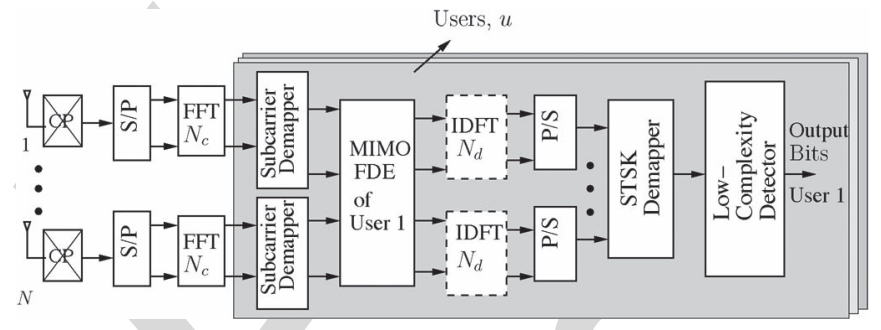

Fig. 16. Receiver model of the SC-FDMA aided STSK scheme. In OFDMA the dotted "IDFT $N_{d}$ " block of the receiver does not exist. Since a single dispersion matrix is selected in each transmission block, a low-complexity single-stream ML detector can be employed.

in Figs. 13 and 14, respectively. As expected, the MC-CDMA STSK scheme is capable of maintaining its superior multiple antenna gain in both the rural area (RA) scenario modeled by the COST207-RA channel as well as in the TU scenario characterized by the COST207-TU12 model. Furthermore, the single-user MC-CDMA aided STSK scheme shows improved performance upon increasing $S_{f}$ both for the COST207-RA and COST207TU12 channels owing to the fact that a FD spreading sequence may still remain recoverable, even when some of its chips are corrupted, while the BER is degraded under multiuser scenarios as a result of the increased MUI imposed by multiple users.

\section{OFDMA/SC-FDMA-Aided Multiuser STSK}

An OFDMA/SC-FDMA-aided STSK scheme was also conceived in [13] which is capable of attaining a superb diversitymultiplexing trade-off even in a multipath environment, whilst additionally supporting multiuser transmissions and maintaining a low peak-to-average power ratio (PAPR) in uplink SCFDMA-aided STSK scenarios. Since OFDMA and SC-FDMA have been adopted for the DL and the UL of the Long Term Evolution (LTE)-Advanced standard, respectively [135], [136], OFDMA/SC-FDMA assisted STSK systems are advocated in [13].

1) Transmitter and Receiver Model of the OFDMA/SCFDMA-Aided STSK: The transmitter and the receiver architectures of an OFDMA/SC-FDMA STSK system are depicted in Figs. 15 and 16, respectively. As shown in Fig. 15, we employ frame based transmission after generating the space-time blocks $\boldsymbol{x}^{u}\left[n_{d}\right]$ for a particular user $u$. In particular, $N_{c}$ 


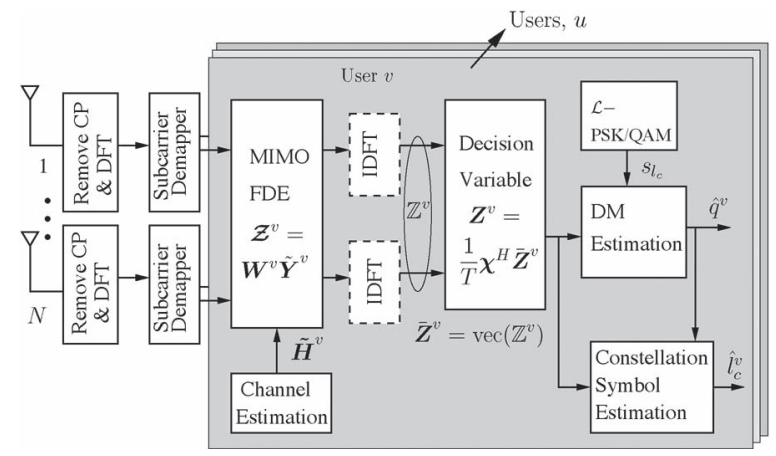

Fig. 17. The receiver architecture of the OFDMA/SC-FDMA-aided STSK scheme employing the reduced-complexity detector of [137]. The dotted "IDFT" block does not exist in the OFDMA-aided STSK receiver.

sub-carriers are used for transmitting a frame, each frame consisting of $N_{d}$ STSK blocks. To be more specific, the data stream $\tilde{\boldsymbol{x}}_{m, T_{i}}^{u}$ to be transmitted from the transmit AE $m$ at a specific time interval $T_{i}$ is first DFT-precoded by the $N_{d}$-point DFT block; in case of OFDMA, however, this step is not required. Then the FD symbols output from the $N_{d}$-point DFT block of the SC-FDMA STSK scheme (or the direct FD STSK codeword symbols of the OFDMA STSK scheme) are mapped to $N_{c}$ subcarriers with $N_{c}=\left(N_{d} \times U\right)$, where the sub-carrier allocation may be in contiguous (localized FDMA or LFDMA) [128] or in an interleaved (IFDMA) [128] fashion. Assuming perfect synchronization at the receiver of Fig. 16 and after removing the $\mathrm{CP}$, the received signal is first Fourier transformed and the resultant FD subcarrier signals are then demapped and equalized in FD by the "MIMO FDE block" of Fig. 16. Following MIMO FDE, the received signal is passed through the " $N_{d}$-point IDFT" block of the desired user $v$ and detected in TD using the single-stream ML detector of [13]. In case of OFDMA, however, " $N N_{d}$-point IDFT" does not exist and the signal is detected in FD by the single-stream ML detector of [13] and as such, the OFDMA scheme cannot benefit from the potential diversity provided by the DFT based precoding stage. It is worth mentioning at this point that under the idealized assumption of perfect synchronization, perfect orthogonality of the users using different sub-carriers, the OFDMA/SC-FDMAaided STSK scheme is decontaminated from MUI. However, the symbols transmitted by a given user in the context of both the LFDMA and IFDMA scheme with MMSE equalization experience some form of self-interference (SI) [110]. The singlestream based ML detector for estimating the source bits of user $v$ from the decision variable $z^{v}$ of the OFDMA/SC-FDMA aided STSK scheme may be formulated as [13]:

$$
\left(\hat{q}^{v}, \hat{l}_{c}^{v}\right)=\underset{q, l_{c}}{\arg \min }\left\|\bar{z}^{v}\left[n_{d}\right]-\chi \boldsymbol{k}_{q, l_{c}}^{v}\right\|^{2},
$$

where $\boldsymbol{k}_{q, l_{c}}^{v}$ is the equivalent transmit signal vector corresponding to user $v$ at indices $q$ and $l_{c}$. Note that the single-stream ML detector of (21) does not explicitly contain either the FD channel transfer function or the TD channel impulse response. Hence, data estimation using (21) involves a reduced number of multiplications and additions.

2) Reduced-Complexity Detector for the OFDMA/SCFDMA-Aided STSK: In order to further reduce the compu- tational complexity of the OFDMA/SC-FDMA-aided STSK scheme of [13], a reduced-complexity detector was conceived in [137]. The reduced-complexity detector of [137] was designed in the spirit of [48], while exploiting the reducedcomplexity behavior of the single-stream ML detector of [8], [13]. The schematic diagram of the OFDMA/SC-FDMA-aided STSK receiver employing the reduced-complexity detector is shown in Fig. 17. This detector first employs MIMO MMSE FDE, followed by the "IDFT" processing, although the "IDFT" block does not exist in case of the OFDMA-aided STSK. Then the indices of the activated DM, $q^{v}$ and of the constellation symbol, $l_{c}^{v}$ corresponding to a particular user $v$ are estimated.

Following the above-mentioned elaborations on the detector's operation obeying the architecture portrayed in Fig. 17, each of the steps followed by the detector may be summarized as in Algorithm 1.

Algorithm 1: A reduced-complexity detector conceived for OFDMA/SC-FDMA-aided STSK

1) Given the STSK parameters $(M, N, T, Q, \mathcal{L})$ and the DMs in terms of the dispersion character matrix (DCM) $\boldsymbol{\chi}$, the signals received by the AEs are input to MIMO FDE after OFDM demodulation and subcarrier demapping;

2) After multiplication by the MMSE weight matrix $\boldsymbol{W}^{v}$, the signal is passed through the "IDFT" block of Fig. 17. For OFDMA, there is no "IDFT" block, as seen in Fig. 17;

3) The vectorial stacking based linearization of [5] is employed: $\overline{\boldsymbol{Z}}^{v}=\operatorname{vec}\left(\mathbb{Z}^{v}\right)$;

4) The decision variable is then obtained as: $\boldsymbol{Z}^{v}=\frac{1}{T} \boldsymbol{\chi}^{H} \overline{\boldsymbol{Z}}^{v}$;

5) The index $\hat{q}^{v}$ of the DM activated and the index $\hat{l}^{v}$ of the constellation symbol are computed separately;

6) The index $\hat{q}^{v}$ of the DM activated is evaluated as:

$$
\begin{aligned}
\hat{q}^{v}=\underset{q \forall l_{c}^{\prime}}{\arg \max }\left[2 \left\{\left|\mathfrak{R}\left(z_{q}^{v}\right)\right|\left|\mathfrak{R}\left(s_{l_{c}^{\prime}}\right)\right|\right.\right. \\
\left.\left.+\left|\mathfrak{I}\left(z_{q}^{v}\right) \| \mathfrak{I}\left(s_{l_{c}^{\prime}}\right)\right|\right\}-\left|s_{l_{c}^{\prime}}\right|^{2}\right]
\end{aligned}
$$

7) The index $\hat{l}^{v}$ of the constellation symbol is found by evaluating:

$$
\hat{l}^{v}=\underset{l}{\arg \min }\left|z_{\hat{q}}^{v}-s_{l}\right|
$$

8) Thereby the source bits of user $v$ have now been estimated.

The reduced-complexity detector is a promising candidate for employment in OFDMA/SC-FDMA-aided STSK transmissions over frequency-selective channels even under multiuser scenarios in terms of its performance versus complexity trade-off.

\section{SucCessive RELAYING-Aided DisTRIBUTED MC STSK}

In Sections III-B, IV-B, and IV-C, we have investigated the schemes incorporating OFDM, MC-CDMA, and OFDMA/ SC-FDMA based transmissions of the multiple-antenna signals 


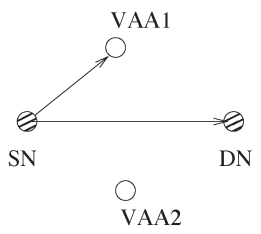

(a)

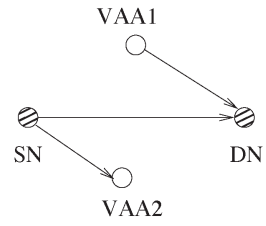

(b)

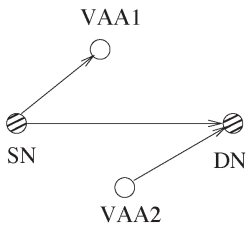

(c)
Fig. 18. Transmission protocol of SR aided cooperation during different time slots [14], [19].

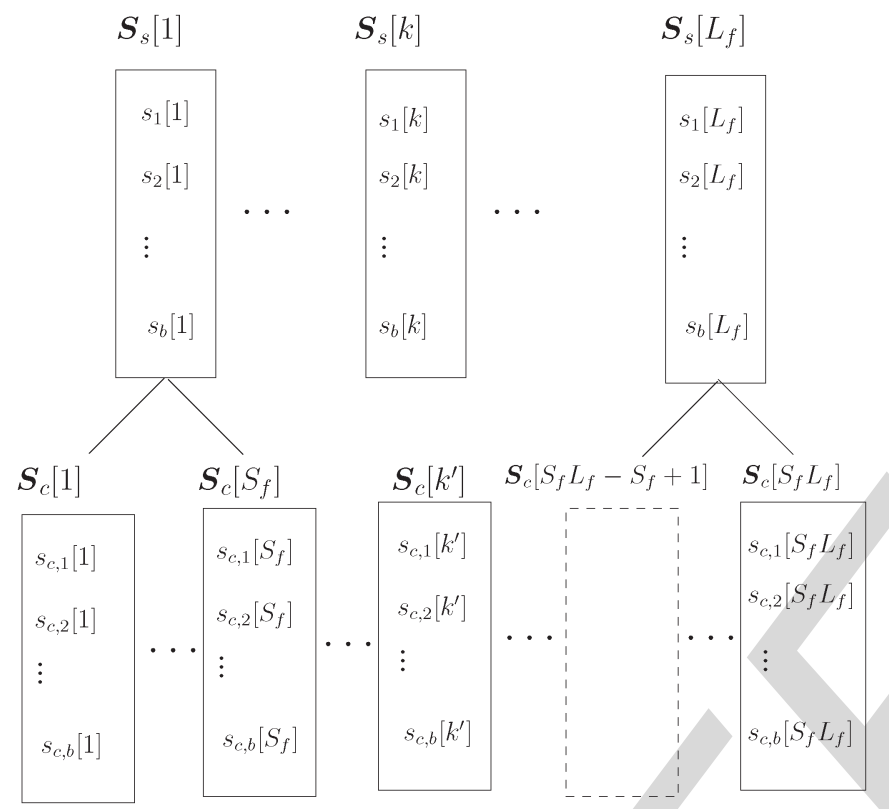

Fig. 19. Illustration of the transmission protocol of the SN of Fig. 18. The $L_{f}$ transmit blocks $S_{S}[k]$ of a transmission frame are spread in FD by the MCCDMA modem using the user-specific spreading sequence $\boldsymbol{C}_{A}^{u}$ or $\boldsymbol{C}_{B}^{u}$ depending on whether frame-A or frame-B is being transmitted, which results in $S_{f} L_{f}$ spread blocks $\boldsymbol{S}_{c}\left[k^{\prime}\right]$.

relying on the STSK system philosophy. However, in those sections, we have focussed our attention on MIMO scenarios, where multiple co-located antennas are employed both at the transmitter as well as at the receiver. Hence for simplicity we refer to these as the family of co-located MIMO schemes, which are potentially susceptible to the detrimental effects of inter-antenna correlation due to the insufficient separation among the antennas. For the sake of mitigating the deleterious effects imposed by the inter-antenna correlation, in this section, we consider SR aided coherent-versus-noncoherent MC STSK schemes.

\section{A. Coherent SR Aided Cooperative MC STSK}

The typical transmission protocol of the SR scheme [19] is shown in Fig. 18, where the distributed RNs form a pair of virtual antenna arrays (VAAs) and the source node (SN) successively transmits its FD spread signals to one of the VAAs in addition to directly transmitting it to the destination node (DN).

1) Transmission Protocol of the $S N$ : The transmission regime of the $\mathrm{SN}$ is illustrated in Fig. 19. The SN first attaches the cyclic redundancy checking (CRC) bits to its information bits and transmits them both to the $M$ RNs

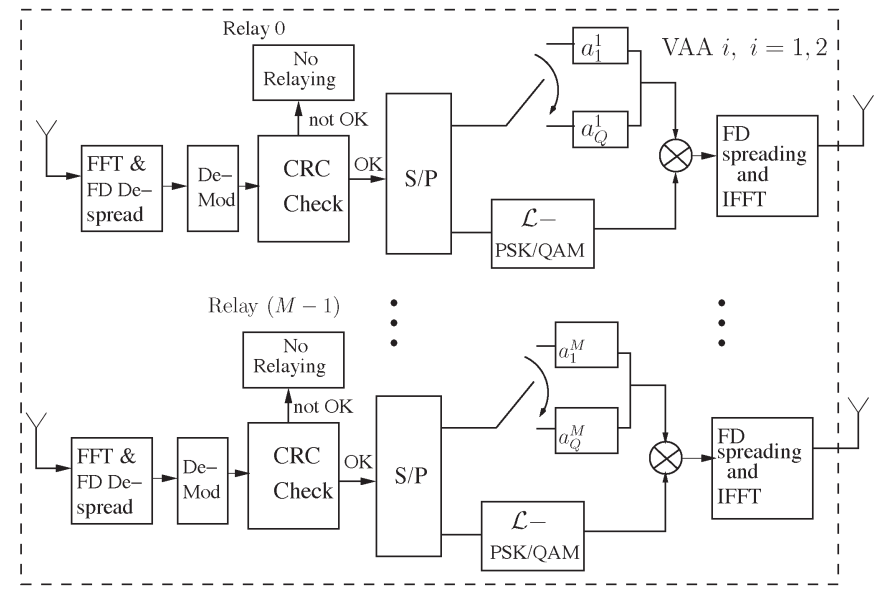

Fig. 20. Architecture and relaying principle of each of the VAAs.

of a VAA as well as to the DN in each of its broadcast phases, as seen from Fig. 19. The CRC-protected bits are then mapped to $\mathcal{L}^{\prime}$-PSK/QAM symbols, which then form the symbol blocks $\boldsymbol{S}_{S}[k] \triangleq\left[s_{1}[k], \ldots, s_{b}[k]\right]^{T} \in \mathbb{C}^{b \times 1}$ of $b$ symbols, where $k(1,2, \ldots)$ denotes the block index. A frame is then defined by $L_{f}$ number of the above-mentioned symbol blocks, where $L_{f}$ represents the frame length. The frames are then divided into two groups-those that are broadcast when VAA1 of Fig. 18 is receiving are termed as frame- $\mathrm{A}$, while those being broadcast when VAA2 is receiving are referred to as frame-B. Frame-A is then spread in FD by the $S_{f}$-length user-specific spreading sequence $\boldsymbol{C}_{A}^{u}$, while frame-B by $\boldsymbol{C}_{B}^{u}$. This spreading operation results in the spread blocks $\boldsymbol{S}_{C}\left[k^{\prime}\right]=C_{A}^{u}\left[s_{f}\right] \boldsymbol{S}_{S}[k] \in \mathbb{C}^{b \times 1}$ and $\boldsymbol{S}_{c}\left[k^{\prime}\right]=C_{B}^{u}\left[s_{f}\right] \boldsymbol{S}_{s}[k] \in \mathbb{C}^{b \times 1}$, respectively, depending on which frame is being transmitted. Assuming the spread frame length $\left(L_{f} \times S_{f}\right)$ to be a multiple of the number $N_{c}$ of subcarriers, each frame is mapped to the subcarriers using the $N_{c}$-point IDFT and then it is transmitted after appending the CPs.

2) The VAA: The constituent RNs of each VAA activate CRC based selective decode-and-forward (DF) relaying. The relaying principle of each of the VAAs is portrayed in Fig. 20. Each of the two VAAs is composed of $M$ RNs and the signal received at each RN of a VAA is decoded employing FD MCCDMA despreading. If the signal at the $\mathrm{RN}$ is deemed to be correctly decoded by the CRC, then it is allowed to relay. The RN then re-encodes the decoded bits to STSK codewords in accordance with the relationship of $b \cdot \log _{2} \mathcal{L}^{\prime}=\log _{2}(\mathcal{L} \cdot Q)$, where $\mathcal{L}$ is the modulation order of the constellation employed by the STSK [14]. In Fig. 20, the $m$-th row of the DM $\boldsymbol{A}_{q}(q=$ $1, \ldots, Q)$ is represented by $a_{q}^{m}$. After appropriate re-encoding, the $m$-th RN transmits the $m$-th row of the resultant STSK codeword after MC-CDMA based FD-spreading and IDFT operation, provided that no decoding error is identified by the CRC. Otherwise, the RN refrains from relaying.

3) Joint Detection at the $D N$ : The DN then jointly detects the signals received via the $\mathrm{SN}$-to-DN and the VAA-to-DN links using the single-stream based joint maximum-likelihood (ML) detector of [14]. To elaborate a little further, the DN employs the double-frame matched filter of [138] for detection of the two replicas of the same signal. Specifically, a filter matched to 
$C_{A}^{u}$ is employed during frame-A, whereas a filter matched to $C_{B}^{u}$ is employed during the next consecutive frame-B transmission.

The SR regime discussed above assists in recovering the half-duplex throughput loss at the cost of imposing inter-VAA interference. Furthermore, the signal also interferes with other stream arriving at the DN [19], [20]. The problem of InterVAA interference is eliminated by invoking the proposed CRC based selective DF cooperation along with the specific FD despreading regime used, while the interference at the DN is mitigated by using the double-frame-based chip-waveform matched filter of [138].

A close observation of the SR strategy reveals that the two replicas of the same frame arrive at the DN in the consecutive phases through the direct SN-DN link and via the VAA links. Hence, the joint detection is carried out over two consecutive frames of the received signal. The joint ML detector conceived for estimating the bits of frame-A may be formulated as [14], [18]:

$$
\left(\hat{q}[k], \hat{l_{c}}[k]\right)=\underset{q, l_{c}}{\arg \min }\left\{\left\|\boldsymbol{Z}^{A}[k]-\boldsymbol{H}_{J}^{A}[k] \overline{\boldsymbol{S}}_{s}^{q, l_{c}}\right\|^{2}\right\},
$$

where $\|\bullet\|$ represents the Euclidean norm of the matrix “ $\bullet$," $\boldsymbol{S}_{s}^{q, l_{c}}$ and $\overline{\boldsymbol{S}}_{s}^{q, l_{c}}$ are the legitimate values of the symbol blocks $\boldsymbol{S}_{s}[k]$ and $\overline{\boldsymbol{S}}_{S}[k]$ specified by the indices $\left(q, l_{c}\right)$ during symbol duration $k$, while the combined FD channel transfer matrix is defined as [14], [18]:

$$
\boldsymbol{H}_{J}^{A}[k] \triangleq\left[\begin{array}{c:c}
\bar{h}_{s d}^{A}[k] \boldsymbol{I}_{b} & \mathbf{0}_{b \times Q} \\
\hdashline \mathbf{0}_{T \times b} & \left.\overline{\boldsymbol{H}}_{v_{1} d}^{A}\left[k+L_{f}\right] \boldsymbol{\chi}\right] \ldots . .
\end{array}\right] \in \mathbb{C}^{(b+T) \times(b+Q)},
$$

with two submatrices expressed by $\bar{h}_{s d}[k] \boldsymbol{I}_{b} \in \mathbb{C}^{b \times b}$ and $\overline{\boldsymbol{H}}_{v_{1} d}\left[k+L_{f}\right] \boldsymbol{\chi} \in \mathbb{C}^{T \times Q}$, respectively, and two zero matrices.

We note that as a benefit of employing SR, the information is received by the DN both from the direct SN-DN link as well as from the VAA-DN link during both the broadcast and the cooperation phase. This leads to the total normalized throughput of [139]

$$
\begin{aligned}
R & =\frac{b \cdot \log _{2} \mathcal{L}^{\prime}+T \log _{2}(\mathcal{L} \cdot Q)}{b+T} \\
& =\frac{2 \log _{2}(\mathcal{L} \cdot Q)}{b+T}[\text { bits/symbol duration }]
\end{aligned}
$$

which is twice that of the corresponding half-duplex scheme [14], [18], [19]. This benefit, however, is achieved at the cost of supporting less users, because the available number of spreading sequences becomes less as a consequence of employing SR.

\section{B. Noncoherent SR Aided Cooperative MC STSK}

In order to overcome the performance degradation imposed by channel estimation (CE) errors, a cooperative MC DSTSK scheme was studied in [14], which retained all the fundamental benefits of the coherent scheme. It was shown in [14] that the CRC-activated coherent scheme potentially benefits from a higher diversity gain than either the conventional DF or the noncooperative schemes, whilst attaining an increased throughput, which is an explicit advantage of using SR. However, the differ- ential scheme suffers from a performance penalty of about $3 \mathrm{~dB}$ compared to the perfect-channel-state information (CSI) aided coherent scheme owing to the inherent noise doubling process of differential encoding. On the other hand, the cooperative coherent scheme's performance was severely degraded by the CE errors. Moreover, the coherent scheme requires the transmission of pilot symbols in addition to the CRC overhead. Hence, the differential multi-carrier cooperative STSK system may be considered an attractive candidate for cooperative MIMO-aided multi-carrier communications.

The transmission regime of the noncoherent MC DSTSK scheme is portrayed in Fig. 21, where the identical architectures of the pair of VAA arrangements are explicitly shown. In particular, the following points are worth mentioning in the context of the distributed MC DSTSK.

1) The DMs used for the cooperative MC DSTSK scheme are directly generated unitary matrices as in [140], so that the employment of nonlinear Cayley transform of [8] may be avoided.

2) The differential encoding requires satisfying the simplifying condition [27] of $M=T$, so that the STSK codewords are $(M \times M)$-element square matrices.

3) Differential encoding of the multicarrier based system can be performed either in the TD (differential encoding across the consecutive symbols of the same sub-carrier) or in the FD (differential encoding across the symbols of the adjacent sub-carriers of the same MC-CDMA block). The TD approach is suitable for continuous transmissions, as opposed to the FD approach, which is useful for burst-based transmissions [141], [142].

\section{ML-MSDSD AND SOFT MAP-MSDSD-AIDED MC DSTSK}

In this section, both hard-decision as well as soft-decision based MSDSD is invoked for MC DSTSK-aided transmission over frequency-selective channels. Specifically, the DSTSK signaling blocks are generated by the original source bits of the hard-decision scheme or by the channel-encoded bits of the soft-decision system. The resultant space-time blocks are then appropriately mapped to a number of OFDM subcarriers. After OFDM-demodulation, the DSTSK signal may be noncoherently detected by a soft-decision-aided MAP-MSDSD detector [69].

Again, as investigated in [8], [14], DSTSK employing conventional differential detection (CDD) suffers from a typical $3 \mathrm{~dB}$ performance penalty in low-Doppler scenarios. Additionally, a high-mobility scenario characterized by a high Doppler frequency may result in an irreducible error floor [143]. The performance erosion of CDD may be mitigated by employing multiple-symbol differential detection (MSDD) [144]. MSDD is capable of efficiently exploiting the fading-plus-noise statistics of the channel for the joint detection of $\left(N_{w}-1\right)$ information symbols from $N_{w}$ number of consecutively received symbols, where $N_{w}$ is usually termed as the observation window size. However, the complexity associated with MSDD is typically high. Hence sphere decoding may be combined with MSDD for reducing the complexity and the resultant scheme is 


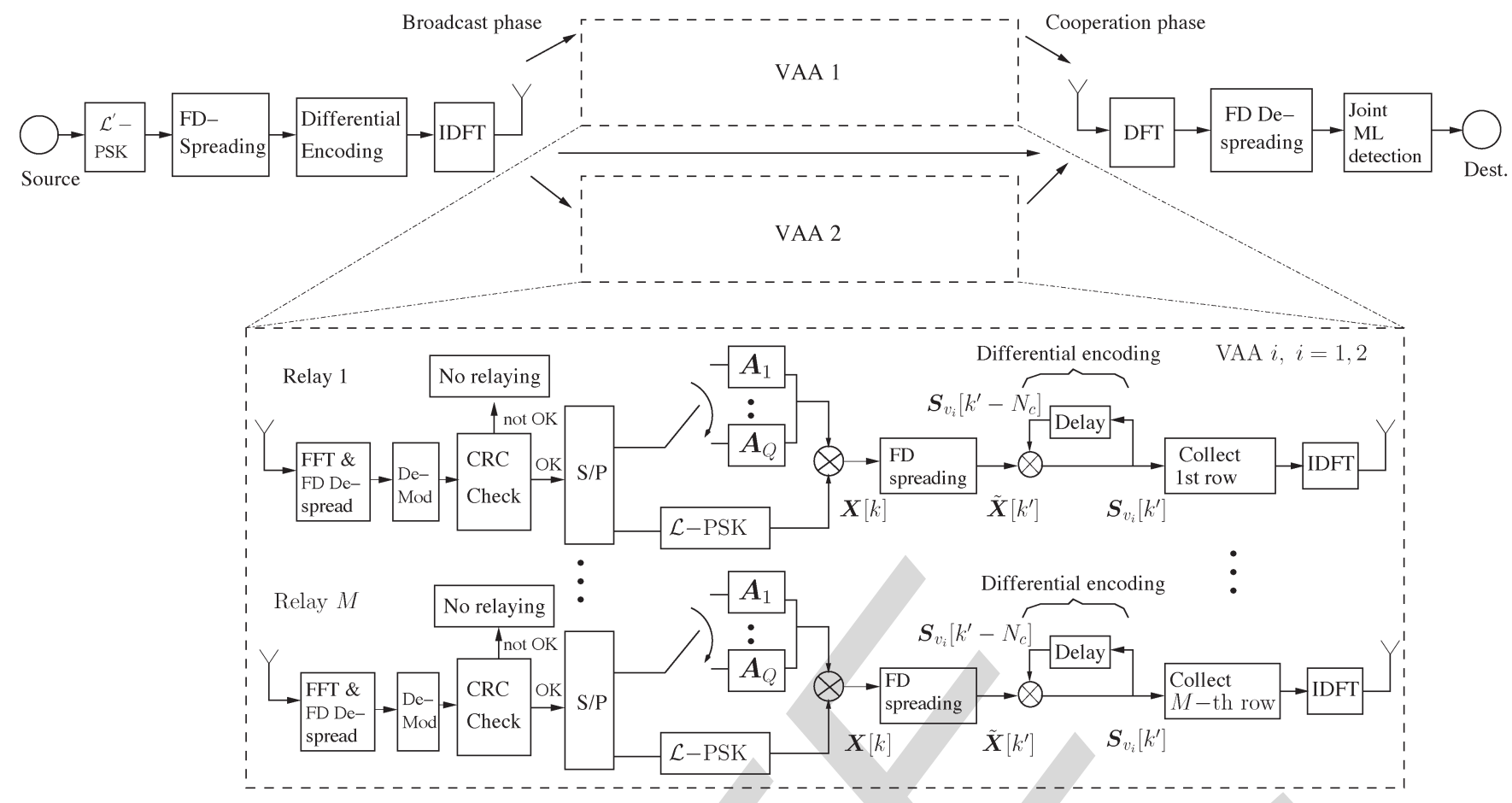

Fig. 21. Schematic of the distributed MC DSTSK employing CRC-based selective DF relaying [139].

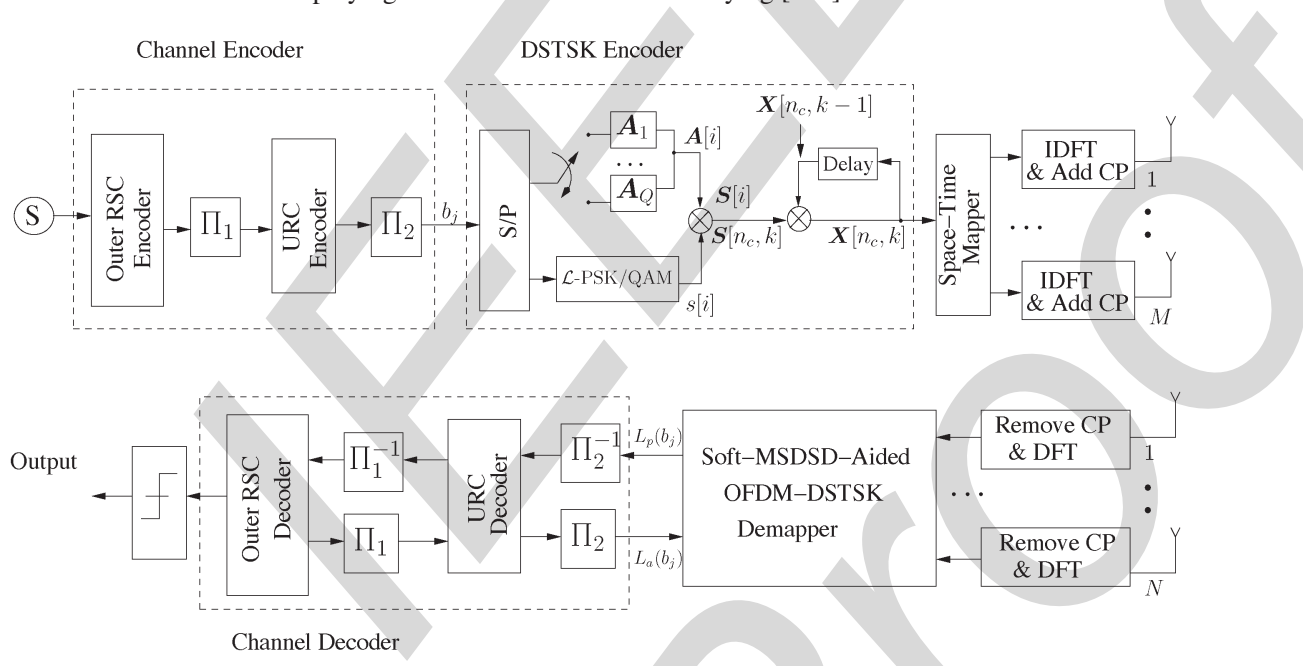

Fig. 22. Transceiver architecture of the concatenated channel-coding aided DSTSK scheme relying on the soft-MSDSD of [69] as the inner decoder. For harddecision scheme, the channel en/decoder block is absent and ML-MSDSD is invoked in place of MAP-MSDSD.

referred to as the MSDSD [145], [146]. On the other hand, the concept of differential space-frequency modulation (DSFM) employing MSDSD was conceived in [141] for exploiting both the achievable spatial- and frequency-domain diversity. Hence, the ML-MSDSD and soft-decision MSDSD designed for OFDM based MC DSTSK operating in frequency-selective channels is investigated in this section [69].

As shown in Fig. 22, the STSK codewords $S[i]$ of the MC DSTSK scheme are mapped to $N_{c}$ parallel subcarriers, before being differentially encoded. The codewords $\boldsymbol{S}\left[n_{c}, k\right]$ are thus differentially encoded to form the transmit blocks $\boldsymbol{X}\left[n_{c}, k\right](k=$ $0,1,2, \ldots)$ according to [141]

$$
\boldsymbol{X}\left[n_{c}, k\right]= \begin{cases}\boldsymbol{X}\left[n_{c}, k-1\right] \boldsymbol{S}\left[n_{c}, k\right], & k=1,2, \ldots \\ \boldsymbol{I}_{T} & k=0 .\end{cases}
$$

The DSTSK codewords are then transmitted after the $N_{c}$ - point inverse discrete Fourier transform (IDFT) operation and appropriate $\mathrm{CP}$ incorporation.

\section{A. ML-MSDSD for MC DSTSK}

The ML-MSDD scheme of [69] processes $N_{w}$ consecutively received space-time blocks of the $n_{c}$-th subcarrier given by $\boldsymbol{Y}^{w}\left[n_{c}, k\right] \triangleq\left[\boldsymbol{Y}^{T}\left[n_{c}, k-N_{w}+1\right], \ldots, \boldsymbol{Y}^{T}\left[n_{c}, k\right]\right]^{T}$ and finds the ML estimates $\boldsymbol{X}^{w}\left[n_{c}, k\right]$ of the corresponding $N_{w}$ transmitted blocks [146], [147], as defined by $\boldsymbol{X}^{w}\left[n_{c}, k\right]=$ $\left[\boldsymbol{X}^{T}\left[n_{c}, k-N_{w}+1\right], \ldots, \boldsymbol{X}^{T}\left[n_{c}, k\right]\right]^{T}$. Since the differentially encoded blocks $\boldsymbol{X}\left[n_{c}, k\right]$ are related to the STSK codewords by a one-to-one mapping relationship, the ML-MSDD in turn estimates the $\left(N_{w}-1\right)$ STSK codewords $S\left[n_{c}, k-N_{w}+2\right]$, 
$\ldots, \boldsymbol{S}\left[n_{c}, k\right]$, which further estimates the source information mapped to the STSK codewords.

For the sake of employing MSDD and in order to reduce the complexity associated with an exhaustive search, we employ ML-MSDSD similar to [145], [146] in order to search through the candidate set lying within a sphere of radius $\rho_{s}[69]$ :

$$
\sum_{\mu=1}^{N_{w}}\left\|\boldsymbol{Y}_{\mu, \mu}^{H} \mathcal{A}_{\mu}+\sum_{v=\mu+1}^{N_{w}}\left(\boldsymbol{Y}_{\mu, v}^{H} \mathcal{A}_{v}\right)\right\|^{2} \leq \rho_{s}^{2},
$$

where the accumulated differential matrices may be expressed as [148]

$$
\mathcal{A}_{v}= \begin{cases}\prod_{\lambda=v}^{N_{w}-1} \boldsymbol{S}_{\lambda}^{H}, & 1 \leqslant v \leqslant\left(N_{w}-1\right) \\ \boldsymbol{I}_{T}, & \boldsymbol{v}=N_{w}\end{cases}
$$

\section{B. Soft-Decision MAP-MSDSD Receiver}

The soft-MSDSD aided MC DSTSK scheme is portrayed in Fig. 22. Assuming the STSK codewords to be mutually independent, the sphere decoding technique of [149], the partial Euclidean distance (PED) criterion detailed in [145], [148], [150] may be invoked. Again, the MAP-MSDSD is initialized with $\mu=\left(N_{w}-1\right)$ and then proceeds by applying the abovementioned PED search criterion until we reach $\mu=1$, where the search radius is updated to $\rho^{2}=d_{1}^{2}$ and the search is repeated by commencing from $\mu=2$ until $\mu=\left(N_{w}-1\right)$ is reached. If the new search does not provide a better estimate, the previous estimate is retained.

Assuming a high interleaver depth so that the permuted bits may be treated as being independent, the a priori loglikelihood ratio (LLR) corresponding to the bit $b_{j}$ interleaved by the interleaver $\Pi_{2}$ of Fig. 22 is defined by [69], [148]: $L_{a}\left(b_{j}\right) \triangleq \ln \frac{P\left(b_{j}=b\right)}{P\left(b_{j}=\bar{b}\right)}$, where $b \in\{0,1\}$ and the $j$-th bit $b_{j}=b$ corresponds to the MAP-MSDSD estimate $\boldsymbol{S}^{\hat{w}}$, while $\bar{b}$ indicates its complement. The a posteriori LLR $L_{p}(\cdot)$ of $b_{j}$ may then be approximated by the maximum-logarithmic-MAP (max-logMAP) algorithm [69], [148]. The extrinsic LLR $L_{e}(\cdot)$ for $b_{j}$ is then evaluated by subtracting the a priori LLR $L_{a}(\cdot)$ from the a posteriori LLR $L_{p}(\cdot)$. The extrinsic information gleaned from the soft-MSDSD demapper is then iteratively exchanged with the URC decoder, which may be referred to as the inner iteration, while the exchange of extrinsic information between the URC and the RSC decoder of Fig. 22 may be referred to as the outer iteration. For each outer iteration between the RSC and the URC decoder, several inner iterations are invoked between the URC and the soft-MSDSD-aided DSTSK demapper [38], [69]. Finally, the bits are estimated from the a posteriori LLRs generated by the RSC decoder.

1) Complexity of the MAP-MSDSD-Aided DSTSK: In order to quantify the complexity of the MAP-MSDSD scheme, we may consider the number of real-valued multiplication operations (RMOs) required for obtaining a single soft-output value, which is used as the complexity metric. The lower bound of the complexity may be obtained [145], [148] as $[M N(2 M+$ 1) $\left./ \log _{2}(\mathcal{L} \cdot Q)+2 M^{2} N\right] N_{w}^{2}$ for large $N_{w}$.

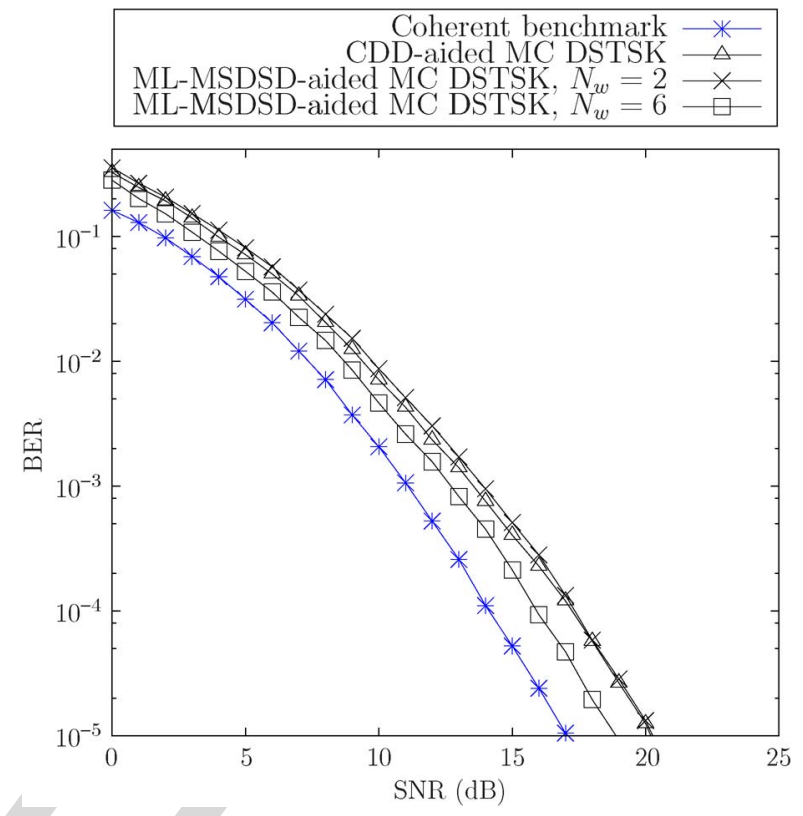

Fig. 23. Simulated BER performance of the ML-MSDSD aided QPSKmodulated MC DSTSK $(2,2,2,4)$ scheme for transmission over dispersive COST207-TU12 channel with normalized Doppler frequency $f_{d}=0.001$ and observation window sizes of $N_{w}=2$ and $N_{w}=6$. The difference in BER performance between coherent and noncoherent scheme decreases with increasing value of $N_{w}$.

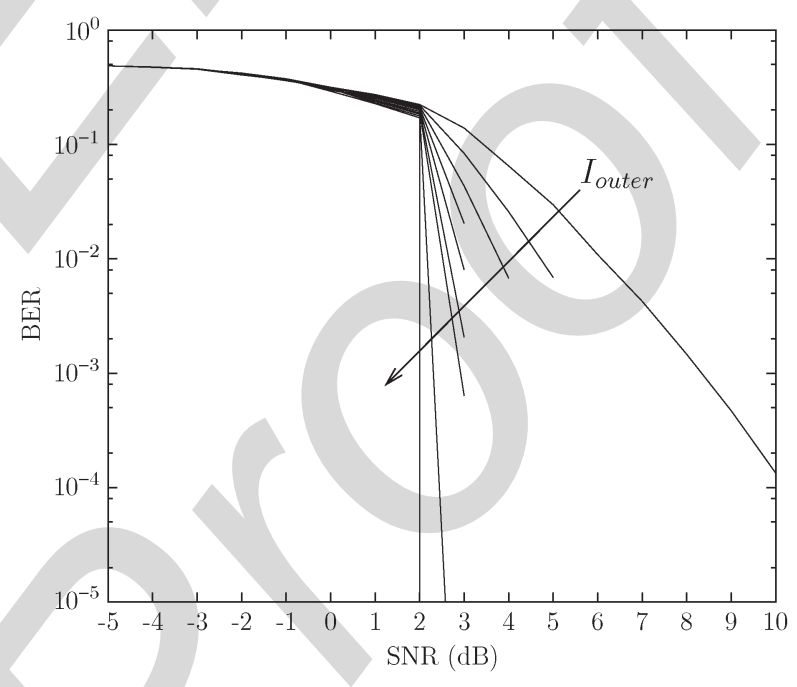

Fig. 24. Achievable BER performance of the soft-MSDSD-aided OFDM $\operatorname{DSTSK}(2,2,2,4)$ in conjunction with QPSK modulation and $N_{w}=4$ for transmission over dispersive COST207-TU12 channel using interleaver length of 200000 bits and a total of 11 outer iterations. Note that after $I_{\text {outer }}=$ 9 iterations, BER converge to vanishingly low BER and the curves are seen almost overlapping after $I_{\text {outer }}=9$ iterations.

2) Performance of the ML-MSDSD and MAP-MSDSDAided DSTSK: Fig. 23 characterizes the achievable BER of the ML-MSDSD-aided QPSK-modulated MC DSTSK $(2,2,2,4)$ scheme, while that of the soft-MSDSD-aided MC DSTSK scheme associated with different observation window sizes of $N_{w}=2,4,6,10$ is investigated in Figs. 24-26. We have employed the COST207-TU12 channel model for the links between each transmit-receive antenna pair and an $\operatorname{RSC}(2,1$, 2) outer code having octrally represented generator polynomials of $\left(g_{r}, g\right)=(3,2)_{8}$ as well as two random interleavers of length 


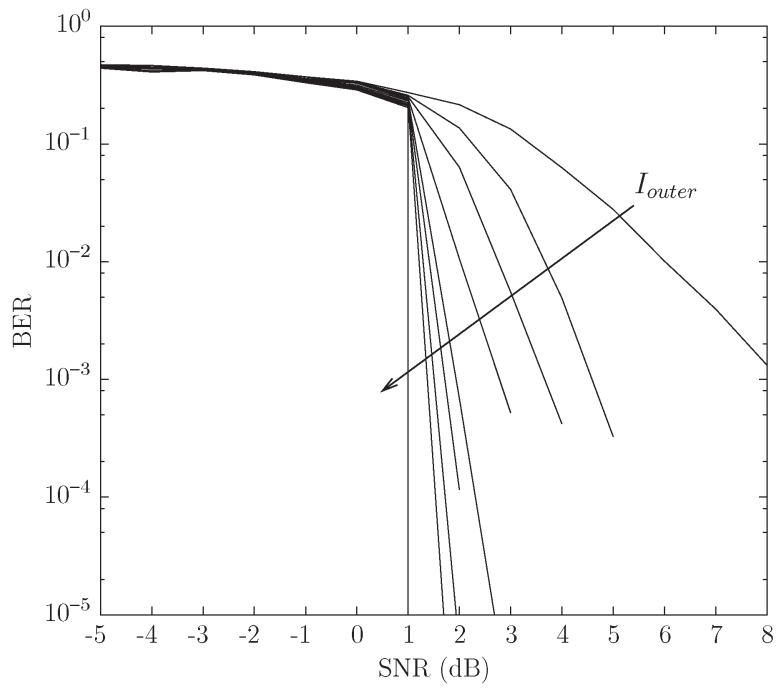

Fig. 25. Achievable BER performance of the soft-MSDSD-aided OFDM DSTSK $(2,2,2,4)$ in conjunction with QPSK modulation and $N_{w}=10$ for transmission over dispersive COST207-TU12 channel using interleaver length of 200000 bits and a total of 11 outer iterations. Note that after $I_{\text {outer }}=$ 9 iterations, BER converge to vanishingly low BER and the curves are seen almost overlapping after $I_{\text {outer }}=9$ iterations.

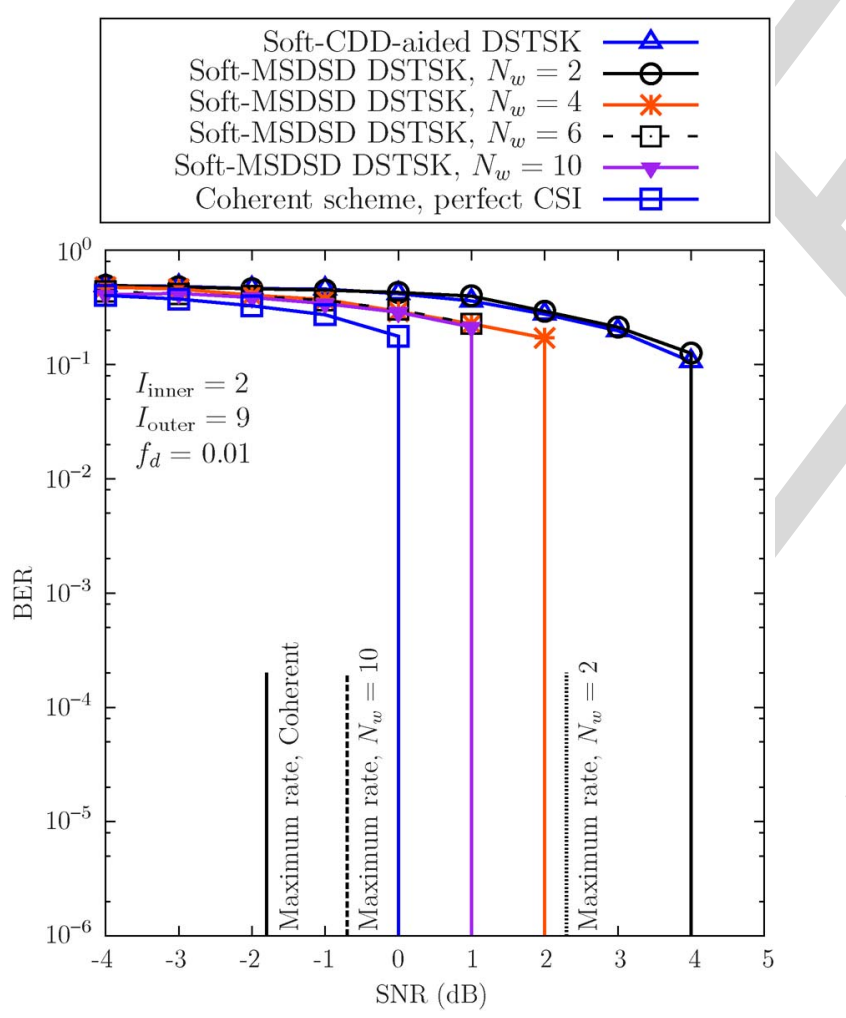

Fig. 26. Simulated BER performance of the soft-MSDSD aided QPSKmodulated MC DSTSK $(2,2,2,4)$ scheme [69] for transmission over dispersive COST207-TU12 channel with normalized Doppler frequency $f_{d}=0.001$ and different observation window size $N_{w}=2,4,6,10$. The BER falls sharply after $I_{\text {outer }}=9$ outer iterations as a benefit of employing the URC and the performance approaches that of the coherent scheme with perfect CSI with increasing value of $N_{w}$.

200000 bits. The number of inner and outer iterations was set to $I_{\text {inner }}=2$ and $I_{\text {outer }}=11$ respectively. As seen in Fig. 23, upon increasing the window size $N_{w}$, the BER performance difference between the coherent and noncoherent scheme is reduced. Figs. 24 and 25 were recorded for the soft-MSDSDaided OFDM DSTSK $(2,2,2,4)$ scheme associated with QPSK modulation and $N_{w}=4$ and $N_{w}=10$, respectively. Observe that the characteristics converge to a low BER for $I_{\text {outer }}=$ 9 iterations. For more than $I_{\text {outer }}=9$ iterations the curves become overlapped. The BER of the ML-MSDSD and softMSDSD-aided MC DSTSK schemes is compared furthermore to that of the corresponding coherent scheme relying on perfect CSI in Figs. 23 and 26. The MC DSTSK scheme has the benefit of dispensing with CE due to differential encoding, while mitigating the dispersion-induced performance erosion of classic STSK by employing OFDM. Furthermore, the multiple-symbol detection partially mitigates the inherent performance penalty imposed by noncoherent detection. Observe in Fig. 26 that as $N_{w}$ increases, the BER performance gradually approaches that of the perfect CSI-oriented coherent scheme. Note that all the performance characteristics converge to a vanishingly low BER after $I_{\text {outer }}=9$ iterations, which is the explicit benefit of using the URC in the system.

\section{Summary AND Potential Future RESEARCH}

In this section, we summarize the philosophy of MC STSK schemes and the relevant design guidelines, followed by a range of research topics concerning potential future studies.

\section{A. Summary}

We have provided detailed transceiver designs for the family of STSK-based MIMO-MC systems for transmissions over dispersive wireless channels. More specifically, OFDM, MCCDMA, OFDMA and SC-FDMA aided MIMO systems employing the STSK concept were discussed. Furthermore, the successive DF relaying aided cooperative MC STSK scheme of [14] was conceived for eliminating the effects of correlated fading often experienced in non-cooperative scenarios, while mitigating the typical throughput loss of classic half-duplex relaying schemes. Furthermore, an OFDM-aided DSTSK arrangement of Fig. 22 was studied for the sake of dispensing with $\mathrm{CE}$, while simultaneously reducing the decoding complexity imposed.

Let us now compare the OFDM-aided coherent STSK (CSTSK) scheme of [11], the MC-CDMA-aided CSTSK regime of [12], the OFDMA/SC-FDMA-aided CSTSK arrangement of [13], the MC-CDMA-aided cooperative CSTSK and DSTSK architecture of [14] and the soft-MSDSD-aided MC DSTSK scheme of [145] in Table VII. We note that all these schemes mitigate the performance degradation imposed by the channel-induced dispersion, but as a further benefit, the MC-CDMA-aided STSK and the SC-FDMA-aided STSK are capable of providing additional FD diversity gains. Under the assumption of perfect orthogonality amongst the subcarriers, the SC-FDMA-aided scheme remains free from MUI, whereas the MUI imposed on the MC-CDMA-aided scheme can only be mitigated by a suitable MUD. The complexity associated with the employment of a MUD is typically high.

The decoding complexity of the different schemes highlighted in this treatise is summarized in Table VIII. In addition 
TABLE VII

Comparative ANALYSis of THE OFDM-Aided CSTSK SCHEME, THE MC-CDMA-AIDED CSTSK SCHEME, THE OFDMA/SC-FDMA-AIDED CSTSK SCHEME, THE MC-CDMA-AIDED COOPERATIVE CSTSK AND DSTSK SCHEMES AND SOFT-MSDSD-AIDED MC DSTSK SCHEME FOR TRANSMISSIONS OVER THE DiSPERSIVE COST 207-TU12 ChANNEL. Co-LoCATED STSK $(2,2,2,4)$, QPSK, AND Distributed STSK $(2,2,4)$, QPSK SCHEMES ARE CONSIDERED

\begin{tabular}{|c|c|c|c|c|c|c|}
\hline & $\begin{array}{r}\text { OFDM- } \\
\text { aided } \\
\text { CSTSK }\end{array}$ & $\begin{array}{r}\text { MC-CDMA- } \\
\text { aided } \\
\text { CSTSK }\end{array}$ & $\begin{array}{r}\text { OFDMA/SC-FDMA-aided } \\
\text { CSTSK }\end{array}$ & $\begin{array}{r}\text { Distributed } \\
\mathrm{MC} \\
\mathrm{CSTSK} \\
\end{array}$ & $\begin{array}{r}\text { Distributed } \\
\mathrm{MC} \\
\text { DSTSK } \\
\end{array}$ & $\begin{array}{r}\text { MSDSD- } \\
\text { aided MC } \\
\text { DSTSK }\end{array}$ \\
\hline $\begin{array}{l}\text { Schematic of } \\
\text { the scheme }\end{array}$ & Fig. 8 & Fig. 11 & $\begin{array}{r}\text { Fig. } 15 \text { and } 16 ; \\
\text { Fig. } 17 \\
\text { (Reduced-complexity detector) }\end{array}$ & $\begin{array}{r}\text { Fig. } 18 \text { and } \\
\text { Fig. } 19\end{array}$ & Fig. 21 & Fig. 22 \\
\hline CSI required? & yes & yes & yes & yes & no & no \\
\hline Detector & $\begin{array}{r}\text { Single- } \\
\text { stream } \\
\mathrm{ML} \\
\text { detector of } \\
\text { (13) }\end{array}$ & $\begin{array}{l}\text { SUD of (20) } \\
\& \text { ML-MUD } \\
\text { of [12] }\end{array}$ & $\begin{array}{r}\text { Single-stream ML detector of } \\
\text { (21) and reduced-complexity } \\
\text { detector of Algorithm } 1 \text { : }\end{array}$ & $\begin{array}{r}\text { Joint ML } \\
\text { detector of } \\
(21)\end{array}$ & $\begin{array}{r}\text { Joint ML } \\
\text { detector of } \\
(24), \text { but } \\
\text { for } \\
\text { differential } \\
\text { scheme } \\
\text { of [14] }\end{array}$ & $\begin{array}{r}\text { ML- } \\
\text { MSDSD } \\
\text { of (28) and } \\
\text { Soft- } \\
\text { decision } \\
\text { MAP- } \\
\text { MSDSD of } \\
\text { Sec- } \\
\text { tion VI-B }\end{array}$ \\
\hline $\begin{array}{l}\text { SNR at } \\
\text { BER }=10^{-5} \\
\text { (uncoded } \\
\text { scheme) }\end{array}$ & $16 \mathrm{~dB}$ & $\begin{array}{r}16 \mathrm{~dB} \\
\left(S_{f}=1\right) \\
13.5 \mathrm{~dB} \\
\left(S_{f}=4\right)\end{array}$ & $\begin{array}{r}16 \mathrm{~dB} \text { (OFDMA, ML) } \\
13.5 \mathrm{~dB} \text { (SC-FDMA, MMSE) }\end{array}$ & $\begin{array}{r}16 \mathrm{~dB} \\
(N=1)\end{array}$ & $\begin{array}{r}19 \mathrm{~dB} \\
(N=1)\end{array}$ & $\begin{array}{r}18.0 \mathrm{~dB} \\
\left(N_{w}=6\right)\end{array}$ \\
\hline
\end{tabular}

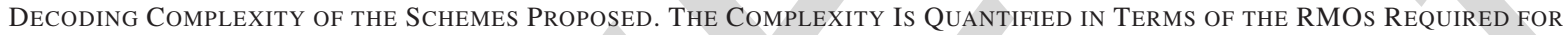
Estimating a Bit or for Obtaining a Single Soft-Output Value of the Soft-MSDSD-Aided MC DSTSK Scheme

\begin{tabular}{|l|r|}
\hline Decoding Scheme & Complexity \\
\hline \hline ML detector of (13) for OFDM-aided CSTSK & $\frac{4 M N T^{2} Q+6 N T Q \mathcal{L}}{\log _{2}(Q \cdot \mathcal{L})}$ \\
\hline The detector of (20) for MC-CDMA-aided CSTSK & $\frac{10 M T Q \mathcal{L}}{\log _{2}(Q \cdot \mathcal{L})}$ \\
\hline ML-MUD of [12] for MC-CDMA-aided CSTSK & $\frac{S_{f}\left(4 M N T^{2} Q U+5 N T Q \mathcal{L} U+2 N T Q \mathcal{L}\right)}{\log _{2}(Q \cdot \mathcal{L})}$ \\
\hline ML detector of [13] for SC-FDMA-aided CSTSK & $\frac{10 M T Q \mathcal{L}}{\log _{2}(Q \cdot \mathcal{L})}$ \\
\hline $\begin{array}{l}\text { Reduced-complexity detector of Algorithm 1: for } \\
\text { OFDMA/SC-FDMA-aided CSTSK }\end{array}$ & $\frac{4 M^{2} N+8 M N+4 M T Q+2 Q \mathcal{L}^{1}+Q+2 \mathcal{L}}{\log _{2}(Q \cdot \mathcal{L})}$ \\
\hline $\begin{array}{l}\text { Joint ML detector of [14] } \\
\text { MAP-MSDSD of Section VI-B for soft-MSDSD-aided } \\
\text { DSTSK }\end{array}$ & $\geq\left[M N(2 M+1) / \log _{2}(\mathcal{L} \cdot Q)+2 M^{2} N\right] N_{w}^{2}$ \\
\hline
\end{tabular}

to the low decoding complexity imposed by the SC-FDMAaided scheme, it has the additional benefit of providing a low PAPR, and hence it is particularly suitable for uplink transmissions. It is also worth noting that the distributed MC STSK scheme of [14] has been investigated in the context of a single DN, but these studies may be readily extended to multiple DNs.

\section{B. Design Guidelines}

The design guidelines of the MC STSK schemes relying on the $(M, N, T, Q)$ parameters in conjunction with the $\mathcal{L}$-ary constellation of the STSK system as a function of the number of subcarriers $N_{c}$, the spreading factor $S_{f}$, the DFT-spreading length $N_{d}$ and the CP-length $L_{\mathrm{cp}}$ are summarized as follows:

1) The values of $(M, N)$ specify the number of transmit and receive AEs.

2) The values of $(Q, \mathcal{L}, T)$, i.e., the number of DMs, the modulation order and the number of symbol intervals over which spreading takes place are chosen in any space-time block so as to satisfy the desirable DMT characterized by the rate $R=\log _{2}(\mathcal{L} \cdot Q) / T$ and by the achievable diversity order of $N \cdot \min (M, N)$.

3) For all the possible combinations of $(\mathcal{L}, Q)$, the $Q \mathrm{DMs}$ are computed based on either the DCMC capacityoptimal or the PEP-optimal design procedure [5], [10]. 
4) The best set of $(\mathcal{L}, Q)$ may be found by predicting the BER performance with the aid of the corresponding EXIT charts.

5) The number of subcarriers is chosen to ensure that the codeword symbols of each subcarrier experience frequency-flat fading. To be specific, the MC modulation scheme is designed by ensuring that the coherence bandwidth of the channel is higher than the subcarrier symbol rate. Additionally, the length of the $\mathrm{CP}$ adopted ensures the absence of ISI between the consecutive OFDM symbols.

6) In the MC-CDMA aided STSK scheme, the number of subcarriers $N_{c}$ has to be equal to or a multiple of the spreading factor $S_{f}$, where increasing $S_{f}$ provides a higher FD diversity gain.

7) In the OFDMA/SC-FDMA aided STSK arrangement, the number of subcarriers $N_{c}$ is usually a multiple of the DFTspreading length $N_{d}$, whereas for a fully-loaded system supporting $U$ users, we have $N_{c}=\left(N_{d} \times U\right)$.

8) In the coherent SR aided cooperative MC-STSK arrangement, the parameters used are designed so as to support the date rate of $R=\frac{2 \log _{2}(\mathcal{L} \cdot Q)}{b+T}$ [bits/symbol duration], where $b$ represents the number of symbols in the SN's transmit blocks.

9) The differential cooperative MC STSK and the softMSDSD aided MC-STSK are designed using directly generated unitary matrices, while satisfying the relationship of $M=T$.

\section{Future Research}

In this section, we briefly discuss a range of potential future research ideas.

1) Asynchronous Transmission: A major challenge of MIMO systems is the IAS problem. The synchronization issue becomes acute in cooperative scenarios due to the asynchronous nature of relay nodes in the network. Perfect synchronization was considered in schemes discussed, although in practice it is difficult to acquire accurate system-level synchronization without imposing a high additional synchronization overhead and implementation complexity. The investigations of [151] revealed that the time-synchronization errors encountered in a cooperative scenario impose a significant performance degradation. Additionally, the propagation delays arising both from the different node locations in a cooperative scheme and from the multipath-induced delay-spread contribute to the sampling offset errors, which further aggravates the effect of synchronization errors. For mitigating the performance degradation imposed by synchronization errors in a cooperative regime, a number of space-time codes were proposed in [152], [153], which invoke space-time equalization under the assumption that the perfect knowledge of the CSI and/or of the relative delays is available at the receiver. In [154], [155], multi-carrier aided STCs were proposed specifically for dispersive links. However, all these schemes suffer from a high computational complexity.

In order to dispense with system-level synchronization between the RF chains associated with the transmit AEs, asynchronous STSK (ASTSK) was proposed in [8], [10]. This scheme operates in a way similar to the SM/SSK systems, because a single $\mathrm{AE}$ is activated at each symbol duration, whilst maintaining the benefits of the STSK system. However, the ASTSK scheme imposes a further constraint on the design of DMs, as discussed in Section II-C1. More specifically, the ASTSK DMs must not contain more than a single non-zero element in each column. Indeed, obtaining suitable DMs which are capable of meeting the required capacity and/or BER performance targets, while satisfying the power constraint mentioned in (5), is a challenging task. The design of a beneficial asynchronous MC STSK arrangement in the context of large-scale (LS) MIMOs [156]-[159] constitutes a set of major open challenges for future research. More specifically, like the SM/SSK schemes, ASTSK has the potential of low-cost hardware design in the context of LS-MIMO systems [70], [160]. Furthermore, the impact of synchronization errors imposed on the cooperative regime of Figs. 20 and 21 and the corresponding design alternative conceived for the asynchronous transmissions has to be investigated.

2) Multiuser Multicell Scenario: The MC STSK scheme relying on both co-located as well as on distributed AEs may be further investigated for employment in multiuser, multicell systems. The scheme may be employed in a coordinated multipoint (CoMP) communications scenario, where the different base stations (BSs) operate in a coordinated manner in order to mitigate the intercell interference and to increase the cell edge throughput [161]-[164]. The scheme may also be considered for employment both in heterogeneous networks (HetNets) including femto/pico BSs, fixed/mobile relays and cognitive radios as well as in appropriate interference mitigation arrangements. The concept of area spectral efficiency (ASE) was conceived in [165] for quantifying the spectral efficiency per unit area of a cellular mobile radio system. In [61], the ASE of soft-decision space-time-frequency-shift keying (STFSK) aided slow-frequency-hopping multiple access (SFHMA) was investigated. Designing appropriate interference management for the schemes of Figs. 20 and 21 under a multiuser multicell scenario constitutes an open problem. A holistically optimized system incorporating the MC STSK scheme may be considered for providing a high ASE.

3) Coded Modulation Aided MC STSK Scheme: The MC STSK scheme may be amalgamated with the family of classic coded modulation schemes, such as Ungerboeck's trellis-coded modulation (TCM) [166]-[168], turbo trellis-coded modulation of Robertson and Worz (TTCM) [169], the bit-interleaved coded modulation (BICM) of Caire et al. [170] and the bit-interleaved coded modulation regime of $\mathrm{Li}$ et al. [171] relying on iterative decoding (BICM-ID). The bandwidthefficient BICM scheme has been widely studied, for example in [172]-[174]. We have employed a near-capacity three-stage coding arrangement for the MC STSK schemes of Fig. 22 and of [12], [13]. These three-stage coding architectures are essentially similar to the BICM technique. However, a comprehensive study of the coded modulation aided MC STSK scheme's performance constitutes a major item of potential future study.

4) Network Coding for Cooperative MC STSK Scheme: Network coding is a promising technique for wireless 
communication systems, which was conceived in the seminal paper by Ahlswede et al. [175] and it is especially beneficial for the sake of increasing the attainable network throughput, the scalability and robustness. Rather than simply replicating and relaying the SN's transmissions at the RNs, the signals are recombined at the intermediate nodes for example by their "Ex-OR" operation or by other linear combination techniques, which are then retransmitted [176], [177]. This was shown to be particularly beneficial in terms of approaching the maximum achievable information flow of the network. Network coding has the potential of improving the robustness, while simultaneously increasing the attainable throughput and reducing the delay of the system. Network coding [178] may be employed in the cooperative MC STSK scheme for further enhancing the ASE. Specifically, the cooperative coherent MC STSK scheme of [14] with its VAAs shown in Fig. 20 and the cooperative MC DSTSK scheme of Fig. 21 may provide increased throughput with the aid of incorporating network coding. However, physical layer network coding and the associated recombining operation at the VAAs would not permit employment of CRC. This is because in a network coded scenario the VAAs will recombine the SN to VAA signal and the inter-VAA interference impinging on them either in the previous or in the next signaling period.

5) Subcarrier Allocation and Scheduling of MC STSK Schemes: We have also the further motivation to design joint power- and sub-carrier allocation schemes for the OFDMA/SCFDMA based STSK DL/UL scenarios. There are numerous popular subcarrier allocation algorithms, such as the Greedy algorithm of Kim and Kim [179], the Hungarian algorithm of Harold [180] and the Worst Subcarrier Avoidance (WSA) technique [181] of Ermolova and Makarevitch, etc. Hence the performance of efficient power and sub-carrier allocation techniques has to be investigated in the context of MC STSK schemes, such as the one proposed by Liu and Yang for lowcomplexity subcarrier-power allocation in [182]. When considering the multiuser, multicell scenarios, appropriate upper layer concepts may be conceived for providing multiuser diversity. Specifically, channel-quality dependent multi-carrier as well as multiuser scheduling has to be invoked for allocating both the spectral and the power resources to different users for the sake of maximizing a meaningful $\mathrm{OF}$, such as for example the total throughput [183]. The performance of different scheduling algorithms also has to be compared. In [184], [185], the authors designed opportunistic relay selection schemes for the SCFDMA uplink. The performance of sophisticated relay selection schemes has to be studied in the context of cooperative multi-carrier STSK.

6) Energy-Efficient MC STSK Design: Against the backdrop of rising energy cost and carbon footprint of cellular networks, "green" wireless systems designed for a high throughput, whilst maintaining a high Quality-of-Service (QoS) and flexible scalability represent a few of the compelling needs of future communication systems. This necessitates the development of power-efficient, low-complexity solutions, whilst satisfying the target QoS as well as the throughput requirements [70], [186]. An appropriate design metric to be invoked may be the normalized energy-efficiency (EE) of Li et al. [187] expressed as the throughput per unit energy. The MC STSK schemes studied in this paper have the potential of considerably reducing the power consumption of wireless systems as a benefit of its inherent diversity-multiplexing gain trade-off. The schemes activate a single DM at any symbol instant and the DMs may be designed for minimizing the overall energy consumption/dissipation. Furthermore, cross-layer-aware upper layer schemes may be designed for supporting energy-efficient operation of the MC STSK schemes.

7) Potential Impact of MC STSK on 4G/5G Wireless-LSMIMO and Millimeter Wave Communications: To cope with the high demand for data services over the Internet, wireless standards are being developed at a breathtaking speed. These standards incorporate all significant improvements in the field of wireless access techniques. For example, wideband code division multiple access (WCDMA) and adaptive modulation and coding (AMC) aided high speed packet access (HSPA) were incorporated in the $3 \mathrm{G}$ specifications. The $4 \mathrm{G}$ concept of the International Mobile Telecommunications-Advanced (IMTAdvanced) system incorporates MIMOs for achieving beneficial multiplexing, diversity and adaptive beamforming gains amalgamated with a full-fledged OFDM and OFDMA-based radio access technology [188], [189]. The 5G system is expected to be rolled out by 2020 for facilitating ubiquitous and seamless connectivity by incorporating techniques such as LS-MIMOs [160], [190], CoMP [162], [191], device-to-device (D2D) communications [192]-[194], energy-efficient systems [195], [196] and millimeter wave (mm-wave) communications [189], [197]. Compared to 4G networks, 5G networks are expected to achieve 1000 times the system capacity and 10 times the spectral efficiency and data rate [196]. This may be achievable with the aid of LS-MIMOs in conjunction with interference-aware multi-tier networks [198], [199] comprising macro cells as well as small cells, relays and D2D networks. Each user of an LS-MIMO system is capable of scaling down its transmit power proportionally to the number of BS antennas [200], thereby providing an improved EE. LS-MIMO systems relying on adaptive beamforming are also compatible with the emerging technique of exploring the underutilized mm-wave frequency spectrum [189], [190], [201], [202]. Rappaport et al. [189] conducted extensive propagation measurements both at $28 \mathrm{GHz}$ as well as at $38 \mathrm{GHz}$ and advocated the inclusion of the unlicensed $\mathrm{mm}$-wave bands in the $5 \mathrm{G}$ cellular landscape.

LS-MIMOs are particularly suitable for the SM scheme, since a high multiplexing gain may be attained at a reduced complexity due to the employment of a limited number of RF chains. However, the practical implementation of SM faces the challenge of acquiring CSI both at the transmitter and at the receiver [70]. The problem of acquiring CSI at the receiver might become acute, when transmit beamforming will be required at the BS. As an impediment, the SM scheme does not provide transmit diversity. By contrast, as a benefit, the OFDMA aided STSK DL and the SC-FDMA aided STSK UL of Section IV-C is readily compatible with the LTE-Advanced physical layer specifications. The MC STSK schemes studied in this treatise also have the potential of supporting LS-MIMO-based 5G networks. The schemes impose low decoding complexity as a benefit of employing single-stream transmission. Hence the number of RF chains may be adaptively selected by a suitable 
design of the STSK DMs. To be more specific, each of the $Q$ DMs, $\boldsymbol{A}_{q},(q=1,2, \ldots, Q)$ may be constructed for ensuring that there is only a low number of non-zero elements in each column of the DMs. This additional constraint imposed on the DM optimization procedure may assist us in using a limited number of RF chains, thereby improving the EE, whilst still retaining the benefits of the STSK scheme. This additional constraint, however, considerably reduces the search space of the set of DMs. The design of efficient DMs for attaining the desired DMT-especially in an LS-MIMO scenario-constitutes an open challenge.

Again, mm-wave techniques constitute promising solutions as part of the HetNet 5G landscape. Due to the decrease in wavelength at these high frequencies, a huge number of halfwavelength-spaced beamforming elements can be accommodated in a limited space, especially at the BS. This renders the MC STSK-based LS-MIMOs a viable solution to attain a flexible DMT at a low complexity and at a low power consumption. In practice, however, mm-wave cellular systems have to compensate for the high free-space path loss. Hence, $5 \mathrm{G}$ cellular systems have to take advantage of the high-dimensional beamforming-aided directional antenna arrays [70], [197], [203], [204]. In the light of this impediment imposed by the use of mm-wave frequencies, the application of MC STSK schemes at these frequencies necessitates practical solutions, which are capable of simultaneously providing diversity, multiplexing and beamforming gains. To the best of our knowledge, the application of MC STSK schemes to the mm-wave band of frequencies has not yet been investigated.

\section{CONCLUSION}

Having commenced with a brief review of MIMO-MC systems and with an introductory discourse on the development of the beneficial STSK system, we provide helpful guidelines for designing MC STSK schemes in this tutorial. Both single-user versus multiuser as well as coherent versus noncoherent $\mathrm{MC}$ STSK were reviewed in this paper. The schemes highlighted here are beneficial in terms of striking a trade-off between the achievable diversity and multiplexing gains, as well as between the performance attained and the complexity imposed, while facilitating communications over dispersive wireless channels. In a nutshell, the basic motivation of the above-mentioned designs was that of improving the overall integrity and throughput of wireless systems by striking a compelling compromise among the conflicting design factors, such as the effective throughput, the coding as well as diversity gain, the implementation complexity etc. To outline the rationale of our work and to summarize our main contributions, we conclude as follows:

- STSK has the benefit of striking a flexible DMT by using single-stream ML detection, as mentioned for example in [8]-[10]. However, all the previous studies apart from [54] investigated STSK in non-dispersive scenarios.

- In order to retain all the benefits of STSK for transmission over dispersive channels, we need either a space-time equalizer or OFDM/MC-CDMA. The latter converts a dispersive channel into reduced-rate parallel non-dispersive channels [11], [12].

- To maintain the benefits of OFDM-based transmission, while facilitating multiuser communications, OFDMA/ SC-FDMA aided STSK systems [13] were proposed. In order to further mitigate the decoding complexity of the single-stream ML detector, a reduced-complexity detector was proposed in [137].

- The transmit power may be reduced with the aid of relaying, especially by DF relaying, where the plausible benefit is that in the absence of decoding errors, the relay retransmits to the DN from a reduced distance.

- Albeit relaying is highly beneficial, it requires both a broadcast and a cooperation slot, which potentially halves the achievable throughput. This disadvantage may be mitigated with the aid of SR, which is achieved at the cost of reducing the number of users supported. Hence, a SRaided coherent versus noncoherent cooperative MC STSK arrangement was studied in [14]. To be specific, the pros and cons of coherent versus noncoherent detection were quantified. Explicitly, in practical terms, it is more realistic to use noncoherent detection dispensing with $\mathrm{CE}$ at the relay nodes.

- Differential STSK employing both hard-decision and softdecision MSDSD were studied for the sake of dispensing with $\mathrm{CE}$ and thus mitigating the potentially high Dopplerdependent pilot overhead, while benefiting both from the low complexity imposed by sphere decoding and from the iterative exchange of soft-information during detection.

\section{Acronyms}

$\mathrm{AE}$

AMC

ASE

ASTSK

BER

BICM

BICM-ID

BLAST

CCMC

CDD

CE

CIR

CoMP

CRC

CSI

CSTSK

D2D

DAB

DCMC

DF

DFT

DL

$\mathrm{DM}$
Antenna Element.

Adaptive Modulation and Coding.

Area Spectral Efficiency.

Asynchronous Space-Time Shift Keying.

Bit Error Ratio.

Bit-interleaved Coded Modulation.

Bit-interleaved Coded Modulation with Iterative Decoding.

Bell Laboratories Layered Space-Time.

Continuous input Continuous output Memory-

less Channel.

Conventional Differential Detection.

Channel Estimation.

Channel Impulse Response.

Coordinated Multipoint.

Cyclic Redundancy Check.

Channel State Information.

Coherent Space-Time Shift Keying.

Device-to-device.

Digital Audio Broadcasting.

Discrete input Continuous output Memoryless Channel.

Decode-and-Forward.

Discrete Fourier Transform.

Downlink.

Dispersion Matrix. 


$\begin{array}{lll}\text { DMT } & \text { Diversity-Multiplexing Tradeoff. } & \text { SUD } \\ \text { DN } & \text { Destination Node. } & \text { TA } \\ \text { DSFM } & \text { Differential Space-Frequency Modulation. } & \text { TCM } \\ \text { DSTSK } & \text { Differential Space-Time Shift Keying. } & \text { TD } \\ \text { DTDR } & \text { Distributed Transmit Directional Receive. } & \text { TTCM } \\ \text { DVB } & \text { Digital Video Broadcasting. } & \text { TU } \\ \text { EE } & \text { Energy Efficiency. } & \text { UL } \\ \text { EGC } & \text { Equal Gain Combining. } & \text { V-BLAST } \\ \text { FD } & \text { Frequency Domain. } & \text { VAA } \\ \text { GA } & \text { Genetic Algorithm. } & \text { WCDMA } \\ \text { HetNet } & \text { Heterogeneous network. } & \text { WINNER } \\ \text { HSPA } & \text { High-Speed Packet Access. } & \text { WSA } \\ \text { IAS } & \text { Inter-Antenna Synchronization. } & \end{array}$

IDFT Inverse Discrete Fourier Transform.

IFDMA Inteleaved Frequency Division Multiple Access. LFDMA Localized Frequency Division Multiple Access. LLR Logarithmic Likelihood Ratio.

LS Large-Scale.

LTE Long Term Evolution.

MAP Maximum a posteriori.

MC Multicarrier.

MC-CDMA Multicarrier Code Division Multiple Access.

MIMO Multiple-Input Multiple-Output.

ML Maximum Likelihood.

MRC Maximal Ratio Combining.

MS Mobile Station.

MSDD Multiple-Symbol Differential Decoding.

MSDSD Multiple-Symbol Differential Sphere Decoding.

MT-CDMA Multitone Code Division Multiple Access.

MUD Multiuser Detector.

MUI Multiuser Interference.

OF Objective Function.

OFDM Orthogonal Frequency Division Multiplexing.

OFDMA Orthogonal Frequency Division Multiple

Access.

$\begin{array}{ll}\text { ORC } & \text { Orthogonality Restoring Combining. } \\ \text { PAPR } & \text { Peak-to-Average Power Ratio. } \\ \text { PEP } & \text { Pairwise Error Probability. } \\ \text { QoS } & \text { Quality-of-Service. } \\ \text { RF } & \text { Radio Frequency. } \\ \text { RMO } & \text { Real-valued Multiplication Operation. } \\ \text { RN } & \text { Relay Node. } \\ \text { SC } & \text { Selection Combining. } \\ \text { SC-FDMA } & \text { Single-Carrier Frequency Division Mult } \\ & \text { Access. } \\ \text { SCM } & \text { Single-Carrier Modulation. } \\ \text { SD } & \text { Spatial Domain. } \\ \text { SDM } & \text { Spatial Division Multiplexing. } \\ \text { SDMA } & \text { Space-Division Multiple Access. } \\ \text { SFHMA } & \text { Slow-Frequency-Hopping Multiple Access. } \\ \text { SM } & \text { Spatial Modulation. } \\ \text { SN } & \text { Source Node. } \\ \text { SR } & \text { Successive Relaying. } \\ \text { SSK } & \text { Space Shift Keying. } \\ \text { STBC } & \text { Space-Time Block Code. } \\ \text { STFSK } & \text { Space-Time-Frequency Shift Keying. } \\ \text { STSK } & \text { Space-Time Shift Keying. } \\ \text { STTC } & \text { Space-Time Trellis Code. }\end{array}$

Single-User Detection.

Transmit Antenna.

Trellis-Coded Modulation.

Time Domain.

Turbo Trellis-Coded Modulation.

Typical Urban.

Uplink.

Vertical Bell Laboratories Layered Space-Time.

Virtual Antenna Array.

Wideband Code Division Multiple Access.

Wireless INitiative NEw Radio.

Worst Subcarrier Avoidance.

\section{REFERENCES}

[1] A. Goldsmith, S. A. Jafar, N. Jindal, and S. Vishwanath, "Capacity limits of MIMO channels," IEEE J. Sel. Areas Commun., vol. 21, no. 5, pp. 684-702, Jun. 2003

[2] E. Telatar, "Capacity of multi-antenna Gaussian channels," Eur. Trans. Telecommun., vol. 10, no. 6, pp. 585-595, Nov./Dec. 1999.

[3] L. Zheng and D. N. C. Tse, "Diversity and multiplexing: A fundamental tradeoff in multiple-antenna channels," IEEE Trans. Inf. Theory, vol. 49, no. 5, pp. 1073-1096, May 2003.

[4] B. Hassibi and B. M. Hochwald, "High-rate codes that are linear in space and time," IEEE Trans. Inf. Theory, vol. 48, no. 7, pp. 1804-1824, Jul. 2002.

[5] R. W. Heath, Jr. and A. J. Paulraj, "Linear dispersion codes for MIMO systems based on frame theory," IEEE Trans. Signal Process., vol. 50, no. 10, pp. 2429-2441, Oct. 2002.

[6] R. Mesleh, H. Haas, S. Sinanovic, C. W. Ahn, and S. Yun, "Spatial modulation," IEEE Trans. Veh. Technol., vol. 57, no. 4, pp. 2228-2241, Jul. 2008.

[7] J. Jeganathan, A. Ghrayeb, L. Szczecinski, and A. Ceron, "Space shift keying modulation for MIMO channels," IEEE Trans. Wireless Commun., vol. 8, no. 7, pp. 3692-3703, Jul. 2009.

[8] S. Sugiura, S. Chen, and L. Hanzo, "Coherent and differential space-time shift keying: A dispersion matrix approach," IEEE Trans. Commun., vol. 58, no. 11, pp. 3219-3230, Nov. 2010.

[9] S. Sugiura, S. Chen, and L. Hanzo, "Generalized space-time shift keying designed for flexible diversity-, multiplexing- and complexity-tradeoffs," IEEE Trans. Wireless Commun., vol. 10, no. 4, pp. 1144-1153, Apr. 2011

[10] S. Sugiura, S. Chen, and L. Hanzo, "A universal space-time architecture for multiple-antenna aided systems," IEEE Commun. Surveys Tuts., vol. 14 , no. 2, pp. 401-420, 2012

[11] M. Driusso, F. Babich, M. I. Kadir, and L. Hanzo, "OFDM aided space-time shift keying for dispersive downlink channels," in Proc. IEEE VTC_Fall, Quebec City, QC, Canada, Sep. 2012, pp. 1-5.

[12] M. I. Kadir, S. Sugiura, S. Chen, and L. Hanzo, "MC-CDMA aided multi-user space-time shift keying in wideband channels," in Proc. IEEE WCNC, Shanghai, China, 2013, pp. 2643-2648.

[13] M. I. Kadir, S. Sugiura, J. Zhang, S. Chen, and L. Hanzo, "OFDMA/ SC-FDMA aided space-time shift keying for dispersive multiuser scenarios," IEEE Trans. Veh. Technol., vol. 62, no. 1, pp. 408-414, Jan. 2013

[14] M. I. Kadir, L. Li, S. Chen, and L. Hanzo, "Successive-relaying-aided decode-and-forward coherent versus noncoherent cooperative multicarrier space-time shift keying," IEEE Trans. Veh. Technol., vol. 62, no. 6, pp. 2544-2557, Jul. 2013.

[15] W. C. Jakes and D. C. Cox, Microwave Mobile Communications. New York, NY, USA: Wiley/IEEE Press, 1994.

[16] J. N. Laneman and G. W. Wornell, "Distributed space-time-coded protocols for exploiting cooperative diversity in wireless networks," IEEE Trans. Inf. Theory, vol. 49, no. 10, pp. 2415-2425, Oct. 2003.

[17] T. Wang, A. Cano, G. B. Giannakis, and J. N. Laneman, "Highperformance cooperative demodulation with decode-and-forward relays," IEEE Trans. Commun., vol. 55, no. 7, pp. 1427-1438, Jul. 2007.

[18] S. Sugiura, S. Chen, H. Haas, P. Grant, and L. Hanzo, "Coherent versus non-coherent decode-and-forward relaying aided cooperative space-time shift keying," IEEE Trans. Commun., vol. 59, no. 6, pp. 1707-1719, Jun. 2011. 
[19] Y. Fan, C. Wang, J. Thompson, and H. V. Poor, "Recovering multiplexing loss through successive relaying using repetition coding," IEEE Trans. Wireless Commun., vol. 6, no. 12, pp. 4484-4493, Dec. 2007.

[20] L. Kong, S. X. Ng, R. G. Maunder, and L. Hanzo, "Near-capacity cooperative space-time coding employing irregular design and successive relaying," IEEE Trans. Commun., vol. 58, no. 8, pp. 2232-2241, Aug. 2010.

[21] J. N. Laneman, D. N. C. Tse, and G. W. Wornell, "Cooperative diversity in wireless networks: Efficient protocols and outage behavior," IEEE Trans. Inf. Theory, vol. 50, no. 12, pp. 3062-3080, Dec. 2004.

[22] D. G. Brennan, "Linear diversity combining techniques," Proc. IEEE, vol. 91 , no. 2, pp. 331-356, Feb. 2003

[23] N. Seshadri and J. H. Winters, "Two signaling schemes for improving the error performance of Frequency Division Duplex (FDD) transmission systems using transmitter antenna diversity," Int. J. Wireless Inf. Netw., vol. 1, no. 1, pp. 49-60, Jan. 1994

[24] S. M. Alamouti, "A simple transmit diversity technique for wireless communications," IEEE J. Sel. Areas Commun., vol. 16, no. 8, pp. 14511458, Oct. 1998.

[25] V. Tarokh, H. Jafarkhani, and A. R. Calderbank, "Space-time block codes from orthogonal designs," IEEE Trans. Inf. Theory, vol. 45, no. 5, pp. 1456-1467, Jul. 1999.

[26] V. Tarokh, N. Seshadri, and A. R. Calderbank, "Space-time codes for high data rate wireless communication: Performance criterion and code construction," IEEE Trans. Inf. Theory, vol. 44, no. 2, pp. 744-765, Mar. 1998.

[27] B. L. Hughes, "Differential space-time modulation," IEEE Trans. Inf. Theory, vol. 46, no. 7, pp. 2567-2578, Nov. 2000.

[28] V. Tarokh and H. Jafarkhani, "A differential detection scheme for transmit diversity," IEEE J. Sel. Areas Commun., vol. 18, no. 7, pp. 11691174, Jul. 2000.

[29] A. J. Paulraj and T. Kailath, "Increasing capacity in wireless broadcast systems using Distributed Transmission/Directional Reception (DTDR),” U.S. Patent 5345 599, Sep. 6, 1994.

[30] G. J. Foschini, "Layered space-time architecture for wireless communication in a fading environment when using multiple antennas," Bell Labs. Tech. J., vol. 1, no. 2, pp. 41-59, 1996.

[31] G. J. Foschini, G. D. Golden, R. A. Valenzuela, and P. W. Wolniansky, "Simplified processing for high spectral efficiency wireless communication employing multi-element arrays," IEEE J. Sel. Areas Commun., vol. 17, no. 11, pp. 1841-1852, Nov. 1999

[32] J. H. Winters, J. Salz, and R. D. Gitlin, "The impact of antenna diversity on the capacity of wireless communication systems," IEEE Trans Commun., vol. 42, no. 2-4, pp. 1740-1751, Feb.-Apr. 1994.

[33] L. C. Godara, "Application of antenna arrays to mobile communications. Part I: Performance improvement, feasibility, system considerations," Proc. IEEE, vol. 85, no. 7, pp. 1031-1060, Jul. 1997.

[34] M. Cooper and M. Goldburg, "Intelligent antennas: Spatial division multiple access," in Proc. ArrayComm, Annu. Rev. Commun., 1996, pp. 999-1002.

[35] P. Vandenameele, L. V. D. Perre, M. G. E. Engels, B. Gyselinckx, and H. J. D. Man, "A combined OFDM/SDMA approach," IEEE J. Sel. Areas Commun., vol. 18, no. 11, pp. 2312-2321, Nov. 2000.

[36] H. Kim and J. Chun, "MIMO structure which combines the spatial multiplexing and beamforming," in Proc. IEEE VTC-Spring, Milan, Italy, May 2004, pp. 108-112.

[37] M. El-Hajjar, O. Alamri, J. Wang, S. Zummo, and L. Hanzo, "Layered steered space-time codes using multi-dimensional sphere packing modulation," IEEE Trans. Wireless Commun., vol. 8, no. 7, pp. 3335-3340, Jul. 2009.

[38] L. Hanzo, O. Alamri, M. El-Hajjar, and N. Wu, Near-Capacity MultiFunctional MIMO Systems (Sphere-Packing, Iterative Detection, and Cooperation). Hoboken, NJ, USA: Wiley, 2009.

[39] J. Mietzner, R. Schober, L. Lampe, W. H. Gerstacker, and P. A. Hoeher, "Multiple-antenna techniques for wireless communications-A comprehensive literature survey," IEEE Commun. Surveys Tuts., vol. 11, no. 2, pp. 87-105, 2009.

[40] M. Jiang and L. Hanzo, "Multiuser MIMO-OFDM for next-generation wireless systems," Proc. IEEE, vol. 95, no. 7, pp. 1430-1469, Jul. 2007.

[41] L. C. Godara, "Application of antenna arrays to mobile communications. Part II.: Beam-forming and direction-of-arrival considerations," Proc. IEEE, vol. 85, no. 8, pp. 1195-1245, Aug. 1997.

[42] J. H. Winters, "Smart antennas for wireless systems," IEEE Personal Commun., vol. 5, no. 1, pp. 23-27, Feb. 1998.
[43] L.-L. Yang and L. Hanzo, "Performance of generalized multicarrier DS-CDMA over Nakagami-m fading channels," IEEE Trans. Commun., vol. 50, no. 6, pp. 956-966, Jun. 2002.

[44] Y. A. Chau and S.-H. Yu, "Space modulation on wireless fading channels," in Proc. IEEE VTC-Fall, Atlantic City, NJ, USA, Oct. 2001, pp. 1668-1671.

[45] R. Mesleh, H. Haas, C. W. Ahn, and S. Yun, "Spatial modulationA new low complexity spectral efficiency enhancing technique," in Proc. CHINACOM, Beijing, China, Oct. 2006, pp. 1-5.

[46] R. Mesleh, S. Engelken, S. Sinanovic, and H. Haas, "Analytical SER calculation of spatial modulation," in Proc ISSSTA, Bologna, Italy, Aug. 2008, pp. 272-276.

[47] J. Jeganathan, A. Ghrayeb, and L. Szczecinski, "Spatial modulation: Optimal detection and performance analysis," IEEE Commun. Lett., vol. 12 , no. 8, pp. 545-547, Aug. 2008.

[48] S. Sugiura, C. Xu, S. X. Ng, and L. Hanzo, "Reduced-complexity coherent versus non-coherent QAM-aided space-time shift keying," IEEE Trans. Commun., vol. 59, no. 11, pp. 3090-3101, Nov. 2011.

[49] R. Mesleh, M. D. Renzo, H. Haas, and P. M. Grant, "Trellis coded spatial modulation," IEEE Trans. Wireless Commun., vol. 9, no. 7, pp. 23492361, Jul. 2010.

[50] M. D. Renzo and H. Haas, "Space Shift Keying (SSK-) MIMO over correlated Rician fading channels: Performance analysis and a new method for transmit-diversity," IEEE Trans. Commun., vol. 59, no. 1, pp. 116129, Jan. 2011.

[51] R. Rajashekar, K. V. S. Hari, and L. Hanzo, "Field extension code based dispersion matrices for coherently detected space-time shift keying," in Proc. IEEE GLOBECOM, Houston, TX, USA, Dec. 2011, pp. $1-5$.

[52] E. Basar, U. Aygolu, E. Panayirci, and H. V. Poor, "New trellis code design for spatial modulation," IEEE Trans. Wireless Commun., vol. 10, no. 8, pp. 2670-2680, Aug. 2011.

[53] P. Yang, Y. Xiao, Y. Yu, and S. Li, "Adaptive spatial modulation for wireless MIMO transmission systems," IEEE Commun. Lett., vol. 15, no. 6, pp. 602-604, Jun. 2011.

[54] H. A. Ngo, C. Xu, S. Sugiura, and L. Hanzo, "Space-time-frequency shift keying for dispersive channels," IEEE Signal Process. Lett., vol. 18, no. 3, pp. 177-180, Mar. 2011.

[55] M. D. Renzo, H. Haas, and P. Grant, "Spatial modulation for multipleantenna wireless systems: A survey," IEEE Commun. Mag., vol. 49, no. 11, pp. 182-191, Dec. 2011

[56] P. Som and A. Chockalingam, "Spatial modulation and space shift keying in single carrier communication," in Proc. IEEE 23rd Int. Symp. PIMRC, Sep. 2012, pp. 1962-1967.

[57] E. Basar, U. Aygolu, E. Panayirci, and H. V. Poor, "Super-orthogonal trellis-coded spatial modulation," IET Commun., vol. 6, no. 17 , pp. 2922-2932, Nov. 2012.

[58] P. Yang, Y. Xiao, L. Li, Q. Tang, and S. Li, "An improved matched-filter based detection algorithm for space-time shift keying systems," IEEE Signal Process. Lett., vol. 19, no. 5, pp. 271-274, May 2012.

[59] F. Babich, A. Crismani, M. Driusso, and L. Hanzo, "Design criteria and genetic algorithm aided optimization of three-stage-concatenated space-time shift keying systems," IEEE Signal Process. Lett., vol. 19, no. 8, pp. 543-546, Aug. 2012.

[60] R. Rajashekar, K. V. S. Hari, and L. Hanzo, "Structured dispersion matrices from division algebra codes for space-time shift keying," IEEE Signal Process. Lett., vol. 20, no. 4, pp. 371-374, Apr. 2013.

[61] H. A. Ngo and L. Hanzo, "Area spectral efficiency of soft-decision space-time-frequency shift-keying-aided slow-frequency-hopping multiple access," IEEE Trans. Veh. Technol., vol. 61, no. 3, pp. 1433-1439, Mar. 2012.

[62] P. Zhang, S. Chen, and L. Hanzo, "Differential space-time shift keyingaided successive-relay-assisted decode-and-forward cooperative multiuser CDMA," IEEE Trans. Veh. Technol., vol. 62, no. 5, pp. 2156-2169, Jun. 2013

[63] M. D. Renzo and H. Haas, "On transmit diversity for spatial modulation MIMO: Impact of spatial constellation diagram and shaping filters at the transmitter," IEEE Trans. Veh. Technol., vol. 62, no. 6, pp. 2507-2531, Jul. 2013.

[64] R. Rajashekar, K. V. S. Hari, and L. Hanzo, "Antenna selection in spatial modulation systems," IEEE Commun. Lett., vol. 17, no. 3, pp. 521-524, Mar. 2013.

[65] N. Serafimovski et al., "On the joint optimization of dispersion matrices and constellations for near-capacity irregular precoded space-time shift keying," IEEE Trans. Wireless Commun., vol. 12, no. 1, pp. 380-387, Jan. 2013 
[66] S. Sugiura and L. Hanzo, "On the joint optimization of dispersion matrices and constellations for near-capacity irregular precoded space-time shift keying," IEEE Trans. Wireless Commun., vol. 12, no. 1, pp. 380387, Jan. 2013.

[67] C. Xu, S. Sugiura, S. X. Ng, and L. Hanzo, "Spatial modulation and space-time shift keying: Optimal performance at a reduced detection complexity," IEEE Trans. Commun., vol. 61, no. 1, pp. 206-216, Jan. 2013.

[68] P. Yang, M. Di Renzo, Y. Xiao, S. Li, and L. Hanzo, "Design guidelines for spatial modulation," IEEE Commun. Surveys Tuts., to be published.

[69] M. I. Kadir, S. Chen, K. V. S. Hari, K. Giridhar, and L. Hanzo, "OFDMaided differential space-time shift keying using iterative soft multiplesymbol differential sphere decoding," IEEE Trans. Veh. Technol., vol. 63, no. 8, pp. 4102-4108, Oct. 2014.

[70] M. D. Renzo, H. Haas, A. Ghrayeb, S. Sugiura, and L. Hanzo, "Spatial modulation for generalized MIMO: Challenges, opportunities and implementation," Proc. IEEE, vol. 102, no. 1, pp. 56-103, Jan. 2014.

[71] S. X. Ng and L. Hanzo, "On the MIMO channel capacity of multidimensional signal sets," IEEE Trans. Veh. Technol., vol. 55, no. 2, pp. 528536, Mar. 2006.

[72] M. Jiang and L. Hanzo, "Unitary linear dispersion code design and optimization for MIMO communication systems," IEEE Signal Process. Lett., vol. 17, no. 5, pp. 497-500, May 2010.

[73] K. A. D. Jong, Evolutionary Computation-A Unified Approach. Cambridge, MA, USA: MIT Press, 2006.

[74] S. Sugiura, "Dispersion matrix optimization for space-time shift keying," IEEE Commun. Lett., vol. 15, no. 11, pp. 1152-1155, Nov. 2011.

[75] M. Patzold, Mobile Fading Channels. New York, NY, USA: Wiley, 2003.

[76] R. Steele and L. Hanzo, Mobile Radio Communications, 2nd ed. New York, NY, USA: Wiley, 1999.

[77] COST 207, "Digital land mobile radio communications, final report," Office Official Publ. Eur. Commun., Luxembourg City, Luxembourg, Tech. Rep., 1989.

[78] T. Hwang, C. Yang, G. Wu, S. Li, and G. Y. Li, "OFDM and its wireless applications: A survey," IEEE Trans. Veh. Technol., vol. 58, no. 4, pp. 1673-1694, May 2009.

[79] G. L. Stuber et al., "Broadband MIMO-OFDM wireless communications," Proc. IEEE, vol. 92, no. 2, pp. 271-294, Feb. 2004.

[80] H. Sampath, S. Talwar, J. Tellado, V. Erceg, and A. Paulraj, "A fourthgeneration MIMO-OFDM broadband wireless system: Design, performance, field trial results," IEEE Commun. Mag., vol. 40, no. 9, pp. 143149, Sep. 2002.

[81] H. Bolcskei, "MIMO-OFDM wireless systems: Basics, perspectives, challenges," IEEE Wireless Commun., vol. 13, no. 4, pp. 31-37, Aug. 2006.

[82] Radio Broadcasting Systems: Digital Audio Broadcasting (DAB) to Mobile, Portable, and Fixed Receivers, ETSI EN 300 401, May 1997.

[83] Digital Video Broadcasting: Framing Structure, Channel Coding, Modulation for Digital Terrestrial Television, ETSI EN 300 744, Aug. 1997.

[84] IEEE Standard for Wireless LAN Medium Access Control (MAC) and Physical (PHY) Layer Specifications, IEEE Std. 802.11, Nov. 1997.

[85] Radio Equipment and Systems, High PErformance Radio Local Area Network (HYPERLAN) Type 1, ETSI ETS 300 652, Oct. 1996.

[86] R. W. Chang, "Synthesis of band-limited orthogonal signals for multichannel data transmission," Bell Syst. Tech. J., vol. 45, no. 10, pp. 17751796, Dec. 1966.

[87] S. Weinstein and P. Ebert, "Data transmission by frequency-division multiplexing using the discrete Fourier transform," IEEE Trans. Commun. Technol., vol. COM-19, no. 5, pp. 628-634, Oct. 1971.

[88] L. Cimini, Jr., "Analysis and simulation of a digital mobile channel using orthogonal frequency division multiplexing," IEEE Trans. Commun., vol. COM-33, no. 7, pp. 665-675, Jul. 1985.

[89] M. Alard and R. Lassalle, "Principles of modulation and channel coding for digital broadcasting for mobile receivers," EBU Tech. Rev., no. 224, pp. 168-190, Aug. 1987

[90] R. S. Blum, G. L. Ye, J. H. Winters, and Y. Qing, "Improved space-time coding for MIMO-OFDM wireless communications," IEEE Trans. Commun., vol. 49, no. 11, pp. 1873-1878, Nov. 2001.

[91] R. W. Chang, "Orthogonal frequency division multiplexing," U.S. Patent 3488 445, Jan. 6, 1970.

[92] J. M. Cioffi, "A multicarrier primer," Amati Commun. Corp./Stanford Univ., Standford, CA, USA, ANSI T1E1.4 91-157, Nov. 1991.

[93] R. V. Nee and R. Prasad, OFDM for Wireless Multimedia Communications, 1st ed. Norwood, MA, USA: Artech House, 2000.
[94] L. Hanzo, M. Munster, B. J. Choi, and T. Keller, OFDM and MC-CDMA for Broadcasting Multi-User Communications, WLANs and Broadcasting. Hoboken, NJ, USA: Wiley, 2003.

[95] C. Williams, M. A. Beach, and S. Mclaughlin, "Robust OFDM timing synchronisation," Electron. Lett., vol. 41, no. 13, pp. 4-5, Jun. 2005.

[96] H. Chen, W. Gao, and D. Daut, "Spectrum sensing for OFDM systems employing pilot tones," IEEE Trans. Wireless Commun., vol. 8, no. 12, pp. 5862-5870, Dec. 2009.

[97] A. Sahin, I. Guvenc, and H. Arslan, "A survey on multicarrier communications: Prototype filters, lattice structures, implementation aspects," IEEE Commun. Surveys Tuts., vol. 16, no. 3, pp. 1312-1338, 2014.

[98] R. J. Piechocki, P. N. Fletcher, A. R. Nix, C. N. Canagarajah, and J. P. McGeehan, "Performance evaluation of BLAST-OFDM enhanced Hiperlan/2 using simulated and measured channel data," Electron. Lett., vol. 37, no. 18, pp. 1137-1139, May 2001

[99] H. Bolcskei, D. Gesbert, and A. J. Paulraj, "On the capacity of OFDMbased spatial multiplexing systems," IEEE Trans. Commun., vol. 50, no. 2, pp. 225-234, Feb. 2002.

[100] Y. Li, J. H. Winters, and N. R. Sollenberger, "MIMO-OFDM for wireless communications: Signal detection with enhanced channel estimation," IEEE Trans. Commun., vol. 50, no. 9, pp. 1471-1477, Sep. 2002.

[101] A. Stamoulis, S. N. Diggavi, and N. Al-Dhahir, "Intercarrier interference in MIMO OFDM," IEEE Trans. Signal Process., vol. 50, no. 10, pp. 2451-2464, Oct. 2002.

[102] H. El-Gamal, A. R. Hammons, Jr., Y. Liu, M. P. Fitz, and O. Y. Takeshita, "On the design of space-time and space-frequency codes for MIMO frequency-selective fading channels," IEEE Trans. Inf. Theory, vol. 49, no. 9, pp. 2277-2292, Sep. 2003

[103] J. H. Moon, Y. H. You, W. G. Jeon, K. W. Kwon, and H. K. Song, "Peak-to-average power control for multiple-antenna HIPERLAN/2 and IEEE802.11a systems," IEEE Trans. Consum. Electron., vol. 49, no. 4, pp. 1078-1083, Nov. 2003.

[104] D. Huang and K. Letaief, "Symbol-based space diversity for coded OFDM systems," IEEE Trans. Wireless Commun., vol. 3, no. 1, pp. 117 127, Jan. 2004.

[105] W. Su, Z. Safar, and K. J. R. Liu, "Full-rate full-diversity spacefrequency codes with optimum coding advantage," IEEE Trans. Inf. Theory, vol. 51, no. 1, pp. 229-249, Jan. 2005.

[106] M. Borgmann and H. Bolcskei, "Noncoherent space-frequency coded MIMO-OFDM,” IEEE J. Sel. Areas Commun., vol. 23, no. 9, pp. 17991810, Sep. 2005.

[107] T. H. Liew and L. Hanzo, "Space-time trellis and space-time block coding versus adaptive modulation and coding aided OFDM for wideband channels," IEEE Trans. Veh. Technol., vol. 55, no. 1, pp. 173-187, Jan. 2006.

[108] N. I. Miridakis and D. D. Vergados, "A survey on the successive interference cancellation performance for single-antenna and multiple-antenna OFDM systems," IEEE Commun. Surveys Tuts., vol. 15, no. 1, pp. 312 335,2013

[109] A. Al-Dweik, F. Kalbat, S. Muhaidat, O. Filio, and S. M. Ali, "Robust MIMO-OFDM system for frequency-selective mobile wireless channels," IEEE Trans. Veh. Technol., to be published.

[110] L.-L. Yang, Multicarrier Communications. Hoboken, NJ, USA: Wiley, Jan. 2009.

[111] T. Keller and L. Hanzo, "Adaptive multicarrier modulation: A convenient framework for time-frequency processing in wireless communications," Proc. IEEE, vol. 88, no. 5, pp. 611-640, May 2000.

[112] L. D’Orazio, C. Sacchi, M. Donelli, J. Louveaux, and L. Vandendorpe, "A near-optimum multiuser receiver for STBC MC-CDMA systems based on minimum conditional BER criterion and genetic algorithmassisted channel estimation," EURASIP J. Wireless Commun. Netw., vol. 2011, no. 1, p. 351 494, Mar. 2011. [Online]. Available: http://jwcn. eurasipjournals.com/content/2011/1/351494

[113] G. Fettweis, J. P. Linnartz, and N. Yee, "Multi-carrier CDMA in indoo wireless radio networks," in Proc. IEEE PIMRC, Yokohama, Japan, Sep. 1993, pp. 109-113.

[114] A. Chouly, A. Brajal, and S. Jourdan, "Orthogonal multicarrier techniques applied to direct sequence spread spectrum CDMA systems," in Proc. IEEE GLOBECOM, Houston, TX, USA, Nov./Dec. 1993, pp. 1723-1728.

[115] V. D. Silva and E. S. Sousa, "Performance of orthogonal CDMA codes for quasi-synchronous communication systems," in Proc. IEEE ICUPC, Ottawa, ON, Canada, Oct. 1993, pp. 995-999.

[116] L. Vandendorpe, "Multitone direct sequence CDMA system in an indoor wireless environment," in Proc. IEEE 1st Symp. Commun. Veh. Technol. Benelux, Delft, The Netherlands, Oct. 1993, pp. 4.1.1-4.1.8. 
[117] S. Hara and R. Prasad, "Overview of multi-carrier CDMA," IEEE Commun. Mag., vol. 35, no. 12, pp. 126-133, Dec. 1997.

[118] L.-L. Yang and L. Hanzo, "Multicarrier DS-CDMA: A multiple access scheme for ubiquitous broadband wireless communications," IEEE Commun. Mag., vol. 41, no. 10, pp. 116-124, Oct. 2003.

[119] L.-L. Yang, W. Hua, and L. Hanzo, "A multicarrier DS-CDMA system using both time-domain and frequency-domain spreading," in Proc. IEEE VTC - Fall, Orlando, FL, USA, Oct. 2003, vol. 4, pp. 2426-2430.

[120] L.-L. Yang, W. Hua, and L. Hanzo, "Multiuser detection in multicarrier CDMA systems employing both time-domain and frequency-domain spreading," in Proc. IEEE PIMRC, Beijing, China, Sep. 2003, vol. 2, pp. $1840-1844$

[121] C.-M. Yang, P.-H. Lin, G.-C. Yang, and W. C. Kwong, "2D orthogonal spreading codes for multicarrier DS-CDMA systems," in Proc. IEEE ICC, Anchorage, AK, USA, May 2003, vol. 5, pp. 3277-3281.

[122] G. Berardinelli, L. A. R. Temino, S. Frattasi, M. I. Rahman, and P. Mogensen, "OFDMA vs. SC-FDMA: Performance comparison in local area IMT-A scenarios," IEEE Wireless Commun., vol. 15, no. 5, pp. 64-72, Oct. 2008.

[123] N. Benvenuto, R. Dinis, D. Falconer, and S. Tomasin, "Single carrier modulation with nonlinear frequency domain equalization: An idea whose time has come-Again," Proc. IEEE, vol. 98, no. 1, pp. 69-96, Jan. 2010.

[124] T. Walzman and M. Schwartz, "Automatic equalization using the discrete frequency domain," IEEE Trans. Inf. Theory, vol. IT-19, no. 1, pp. 5968, Jan. 1973.

[125] H. Sari, G. Karam, and I. Jeanclaude, "Transmission techniques for digital terrestrial TV broadcasting," IEEE Commun. Mag., vol. 33, no. 2, pp. 100-109, Feb. 1995.

[126] D. Falconer, S. L. Ariyavisitakul, A. Benyamin-Seeyar, and B. Eidson, "Frequency domain equalization for single-carrier broadband wireless systems," IEEE Commun. Mag., vol. 40, no. 4, pp. 58-66, Apr. 2002.

[127] D. Falconer and S. L. Ariyavisitakul, "Broadband wireless using single carrier and frequency domain equalization," in Proc. 5th Int. Symp. WPMC, Honolulu, HI, USA, Oct. 2002, vol. 1, pp. 27-36.

[128] H. G. Myung, J. Lim, and D. J. Goodman, "Single carrier FDMA for uplink wireless transmission," IEEE Veh. Technol. Mag., vol. 1, no. 3, pp. 30-38, Sep. 2006.

[129] WINNER, "The WINNER II air interface: Refined multiple access concepts," Munich, Germany, IST-4-027756 WINNER II, D4.6.1, Tech. Rep., Nov. 2006.

[130] U. Sorger, I. D. Broeck, and M. Schnell, "Interleaved FDMA-A new spread-spectrum multiple-access scheme," in Proc. IEEE ICC, Atlanta, GA, USA, Jun. 1998, vol. 2, pp. 1013-1017.

[131] N. Benvenuto and S. Tomasin, "On the comparison between OFDM and single carrier modulation with a DFE using a frequency-domain feedforward filter," IEEE Trans. Commun., vol. 50, no. 6, pp. 947-955, Jun. 2002.

[132] A. Wilzeck, Q. Cai, M. Schiewer, and T. Kaiser, "Effect of multiple carrier frequency offsets in MIMO SC-FDMA systems," in Proc. Int. ITG/ IEEE Workshop Smart Antennas, Vienna, Austria, Feb. 2007, pp. 1-7.

[133] C. Ciochina and H. Sari, "A review of OFDMA and single-carrier FDMA," in Proc. EW, Lucca, Italy, Apr. 2010, pp. 706-710.

[134] V. Dalakas, P. T. Mathiopoulos, F. D. Cecca, and G. Gallinaro, "A comparative study between SC-FDMA and OFDMA schemes for satellite uplinks," IEEE Trans. Broadcast., vol. 58, no. 3, pp. 370-378, Sep. 2012.

[135] A. Ghosh, R. Ratasuk, B. Mondal, N. Mangalvedhe, and T. Thomas, "LTE-Advanced: Next-generation wireless broadband technology," IEEE Commun. Mag., vol. 17, no. 3, pp. 10-22, Jun. 2010.

[136] F. Boccardi et al., "Multiple-antenna techniques in LTE-Advanced," IEEE Commun. Mag., vol. 50, no. 3, pp. 114-121, Mar. 2012.

[137] M. I. Kadir, S. Chen, and L. Hanzo, "A reduced-complexity detector for OFDMA/SC-FDMA-aided space-time shift keying," in Proc. IEEE VTC - Fall, Las Vegas, NV, USA, Sep. 2013, pp. 1-5.

[138] L. Li and L. Hanzo, "Multiple-symbol differential sphere detection aided successive relaying in the cooperative DS-CDMA uplink," in Proc. IEEE WCNC, Cancun, Mexico, Mar. 2011, pp. 1875-1880.

[139] M. I. Kadir, "Co-located and distributed multicarrier space-time shift keying for wideband channels," Ph.D. dissertation, Univ. Southampton, Southampton, U.K., 2014.

[140] C. Xu, S. Sugiura, S. X. Ng, and L. Hanzo, "Reduced-complexity noncoherently detected differential space-time shift keying," IEEE Signal Process. Lett., vol. 18, no. 3, pp. 153-156, Mar. 2011.
[141] V. Pauli, L. Lampe, and J. Huber, "Differential space-frequency modulation and fast 2-D multiple-symbol differential detection for MIMOOFDM," IEEE Trans. Veh. Technol., vol. 57, no. 1, pp. 297-310, Jan. 2008.

[142] L. Wang, "Low-complexity near-optimum detection techniques for noncooperative and cooperative MIMO systems," Ph.D. dissertation, Univ. Southampton, Southampton, U.K., 2010.

[143] L. Hanzo, Y. Akhtman, L. Wang, and M. Jiang, MIMO-OFDM for LTE, WIFI and WIMAX: Coherent Versus Non-Coherent and Cooperative Turbo-Receivers. Hoboken, NJ, USA: Wiley, 2001.

[144] D. Divsalar and M. K. Simon, "Multiple-symbol differential detection of MPSK," IEEE Trans. Commun., vol. 38, no. 3, pp. 300-308, Mar. 1990

[145] L. Lampe, R. Schober, V. Pauli, and C. Windpassinger, "Multiple-symbol differential sphere decoding," IEEE Trans. Commun., vol. 53, no. 12, pp. 1981-1985, Dec. 2005.

[146] V. Pauli and L. Lampe, "On the complexity of sphere decoding for differential detection," IEEE Trans. Inf. Theory, vol. 53, no. 4, pp. 15951603, Apr. 2007

[147] V. Pauli and L. Lampe, "Tree-search multiple-symbol differential decoding for unitary space-time modulation," IEEE Trans. Commun., vol. 55, no. 8, pp. 1567-1576, Aug. 2007.

[148] V. Pauli, L. Lampe, and R. Schober, “"Turbo DPSK” using soft multiplesymbol differential sphere decoding," IEEE Trans. Inf. Theory, vol. 52, no. 4, pp. 1385-1398, Apr. 2006.

[149] E. Agrell, T. Eriksson, A. Vardy, and K. Zeger, "Closest point search in lattices," IEEE Trans. Inf. Theory, vol. 48, no. 8, pp. 2201-2214, Aug. 2002.

[150] T. Wiegand, N. Heidmann, and S. Paul, "Modified partial Euclidean distance for iterative tree-search MIMO detection," in Proc. IEEE PIMRC, Toronto, ON, Canada, Sep. 2011, pp. 1723-1727.

[151] R. C. Palat, A. Annamalai, and J. H. Reed, "Accurate bit-error-rate analysis of bandlimited cooperative OSTBC networks under timing synchronization errors," IEEE Trans. Veh. Technol., vol. 58, no. 5, pp. $2191-$ 2200, Jun. 2009

[152] X. Li, "Space-time coded multi-transmission among distributed transmitters without perfect synchronization," IEEE Signal Process. Lett., vol. 11, no. 12, pp. 948-951, Dec. 2004.

[153] Y. Li and X.-G. Xia, "A family of distributed space-time trellis codes with asynchronous cooperative diversity," IEEE Trans. Commun., vol. 55, no. 4, pp. 790-800, Apr. 2007.

[154] Z. Li and X.-G. Xia, "An Alamouti coded OFDM transmission for cooperative systems robust to both timing errors and frequency offsets," IEEE Trans. Wireless Commun., vol. 7, no. 5, pp. 1839-1844, May 2008.

[155] M. Sharp, A. Scaglione, and B. Sirkeci-Mergen, "Randomized cooperation in asynchronous dispersive links," IEEE Trans. Commun., vol. 57, no. 1, pp. 64-68, Jan. 2009.

[156] K. Wu, Y. J. Cheng, T. Djerafi, and W. Hong, "Substrate-integrated millimeter-wave and Terahertz antenna technology," Proc. IEEE, vol. 100, no. 7, pp. 2219-2232, Jul. 2012.

[157] J. Hoydis, S. ten Brink, and M. Debbah, "Massive MIMO in the UL/DL of cellular networks: How many antennas do we need?" IEEE J. Sel. Areas Commun., vol. 31, no. 2, pp. 160-171, Feb. 2013.

[158] L. Hanzo, M. El-Hajjar, and O. Alamri, "Near-capacity wireless transceivers and cooperative communications in the MIMO era: Evolution of standards, waveform design, future perspectives," Proc. IEEE, vol. 99, no. 8, pp. 1343-1385, Aug. 2011.

[159] R. Kudo, S. M. D. Armour, J. P. McGeehan, and M. Mizoguchi, "A channel state information feedback method for massive MIMO-OFDM," J. Commun. Netw., vol. 15, no. 4, pp. 352-361, Aug. 2013.

[160] E. G. Larsson, F. Tufvesson, O. Edfors, and T. L. Marzetta, "Massive MIMO for next generation wireless systems," IEEE Commun. Mag., vol. 52, no. 2, pp. 186-195, Feb. 2014.

[161] Q. Zhang, C. Yang, and A. Molisch, "Downlink base station cooperative transmission under limited-capacity backhaul," IEEE Trans. Wireless Commun., vol. 12, no. 8, pp. 3746-3759, Aug. 2013.

[162] P. Marsch and G. Fettweis, "Uplink CoMP under a constrained backhaul and imperfect channel knowledge," IEEE Trans. Wireless Commun., vol. 10, no. 6, pp. 1730-1742, Jun. 2011.

[163] D. Gesbert et al., "Multi-cell MIMO cooperative networks: A new look at interference," IEEE J. Sel. Areas Commun., vol. 28, no. 9, pp. 13801408, Dec. 2010.

[164] I. Hwang, C.-B. Chae, J. Lee, and R. W. Heath, Jr., "Multicell cooperative systems with multiple receive antennas," IEEE Trans. Wireless Commun., vol. 20, no. 1, pp. 50-58, Feb. 2013. 
[165] M.-S. Alouini and A. J. Goldsmith, "Area spectral efficiency of cellular mobile radio systems," IEEE Trans. Veh. Technol., vol. 48, no. 4, pp. 1047-1066, Jul. 1999.

[166] G. Ungerboeck, "Channel coding with multilevel/phase signals," IEEE Trans. Inf. Theory, vol. IT-28, no. 1, pp. 55-67, Jan. 1982.

[167] G. Ungerboeck, "Trellis-coded modulation with redundant signal sets-Part I: Introduction," IEEE Commun. Mag., vol. 25, no. 2, pp. 511, Feb. 1987.

[168] G. Ungerboeck, "Trellis-coded modulation with redundant signal sets-Part II: State of the art," IEEE Commun. Mag., vol. 25, no. 2, pp. 12-21, Feb. 1987.

[169] P. Robertson and T. Worz, "Bandwidth-efficient turbo trellis-coded modulation using punctured component codes," IEEE J. Sel. Areas Commun., vol. 16, no. 2, pp. 206-218, Feb. 1998

[170] G. Caire, G. Taricco, and E. Biglieri, "Bit-interleaved coded modulation," IEEE Trans. Inf. Theory, vol. 44, no. 3, pp. 927-946, May 1998.

[171] X. Li, A. Chindapol, and J. A. Ritcey, "Bit-interleaved coded modulation with iterative decoding and 8 PSK signaling," IEEE Trans. Commun., vol. 50, no. 8, pp. 1250-1257, Aug. 2002.

[172] L. Lampe and R. Schober, "Bit-interleaved coded differential space-time modulation," IEEE Trans. Commun., vol. 50, no. 9, pp. 1429-1439, Sep. 2002.

[173] C. H. Choi and G. H. Im, "Bit-interleaved coded multilevel modulation for single-carrier frequency-domain equalization," IEEE Commun. Lett., vol. 14, no. 3, pp. 193-195, Mar. 2010.

[174] R. Tee, R. G. Maunder, and L. Hanzo, "EXIT-chart aided near-capacity irregular bit-interleaved coded modulation design," IEEE Trans. Wireless Commun., vol. 8, no. 1, pp. 32-37, Jan. 2009.

[175] R. Ahlswede, N. Cai, S.-Y. Li, and R. W. Yeung, "Network information flow," IEEE Trans. Inf. Theory, vol. 46, no. 4, pp. 1204-1216, Jul. 2000.

[176] Z. Chen, B. Xia, Z. Hu, and H. Liu, "Design and analysis of multi-level physical-layer network coding for Gaussian two-way relay channels," IEEE Trans. Commun., vol. 62, no. 6, pp. 1803-1817, Jun. 2014.

[177] G. Bartoli, R. Fantacci, D. Marabissi, and R. Simoni, "Subcarriers suppression methods for OFDM systems with decode-and-forward network coding," IEEE Trans. Wireless Commun., vol. 12, no. 12, pp. 6034-6042, Dec. 2013.

[178] R. Bassoli, H. Marques, J. Rodriguez, K. W. Shum, and R. Tafazolli, "Network coding theory: A survey," IEEE Commun. Surveys Tuts., vol. 15, no. 4, pp. 1950-1978, 2013.

[179] Y. Kim and J. Kim, "A 2-D subcarrier allocation scheme for capacity enhancement in a clustered OFDM system," IEICE Trans., vol. 90-B, no. 7, pp. 1880-1883, Jul. 2007.

[180] W. K. Harold, "The Hungarian method for the assignment problem," Naval Res. Logist. Quart., vol. 2, no. 1/2, pp. 83-97, Mar. 1955.

[181] N. Y. Ermolova and B. Makarevitch, "Performance of practical subcarrier allocation schemes for OFDMA," in Proc. IEEE PIMRC, Athens, Greece, Sep. 2007, pp. 1-4.

[182] T. Liu, C. Yang, and L.-L. Yang, "A low-complexity subcarrierpower allocation scheme for frequency-division multiple-access systems," IEEE Trans. Wireless Commun., vol. 9, no. 5, pp. 1564-1570, May 2010.

[183] J. Lim, H. G. Myung, K. Oh, and D. J. Goodman, "Channel-dependent scheduling of uplink single carrier FDMA systems," in Proc. IEEE VTC - Fall, Montreal, QC, Canada, Sep. 2006, pp. 1-5.

[184] J. Zhang, L.-L. Yang, and L. Hanzo, "Power-efficient opportunistic amplify-and-forward single-relay aided multi-user SC-FDMA uplink," in Proc. IEEE VTC-Spring, Taipei, Taiwan, May 2010, pp. 1-5.

[185] J. Zhang, L.-L. Yang, and L. Hanzo, "Energy-efficient channeldependent cooperative relaying for the multiuser SC-FDMA uplink," IEEE Trans. Veh. Technol., vol. 60, no. 3, pp. 992-1004, Mar. 2011.

[186] D. Feng et al., "A survey of energy-efficient wireless communications," IEEE Commun. Surveys Tuts., vol. 15, no. 1, pp. 167-178, 2013.

[187] G. Li et al., "Energy-efficient wireless communications: Tutorial, survey, open issues," IEEE Trans. Wireless Commun., vol. 18, no. 6, pp. 28-35, Dec. 2011.

[188] L. Hanzo et al., "Wireless myths, realities, futures: From 3G/4G to optical and quantum wireless," Proc. IEEE, vol. 100, no. Special Centennial Issue, pp. 1853-1888, May 2012.

[189] T. S. Rappaport et al., "Millimeter wave mobile communications for 5G cellular: It will work!" IEEE Access, vol. 1, pp. 335-349, 2013.

[190] F. Rusek et al., "Scaling up MIMO: Opportunities and challenges with very large arrays," IEEE Signal Process. Mag., vol. 30, no. 1, pp. 40-60, Jan. 2013.
[191] G. Y. Li, J. Niu, D. Lee, J. Fan, and Y. Fu, "Multi-cell coordinated scheduling and MIMO in LTE," IEEE Commun. Surveys Tuts., vol. 16, no. 2, pp. 761-775, 2014.

[192] M. N. Tehrani, M. Uysal, and H. Yanikomeroglu, "Device-to-device communication in $5 \mathrm{G}$ cellular networks: Challenges, solutions, future directions," IEEE Commun. Mag., vol. 52, no. 5, pp. 86-92, May 2014.

[193] D. Feng et al., "Device-to-device communications in cellular networks," IEEE Commun. Mag., vol. 52, no. 4, pp. 49-55, Apr. 2014.

[194] V. Jungnickel et al., "The role of small cells, coordinated multipoint, massive MIMO in 5G," IEEE Commun. Mag., vol. 52, no. 5, pp. 44-51, May 2014.

[195] W. H. Chin, Z. Fan, and R. Haines, "Emerging technologies and research challenges for 5G wireless networks," IEEE Wireless Commun., vol. 21, no. 2, pp. 106-112, Apr. 2014.

[196] C.-X. Wang et al., "Cellular architecture and key technologies for 5G wireless communication networks," IEEE Commun. Mag., vol. 52, no. 2 , pp. 122-130, Feb. 2014

[197] T. S. Rappaport, J. N. Murdock, and F. Gutierrez, "State of the art in 60-GHz integrated circuits and systems for wireless communications," Proc. IEEE, vol. 99, no. 8, pp. 1390-1436, Aug. 2011.

[198] E. Hossain, M. Rasti, H. Tabassum, and A. Abdelnasser, "Evolution toward 5G multi-tier cellular wireless networks: An interference management perspective," IEEE Wireless Commun., vol. 21, no. 3, pp. 118127, Jun. 2014

[199] A. Osseiran et al., "Scenarios for 5G mobile and wireless communications: The vision of the METIS project," IEEE Commun. Mag., vol. 52, no. 5, pp. 26-35, May 2014.

[200] H. Q. Ngo, E. G. Larsson, and T. Marzetta, "Energy and spectral efficiency of very large multiuser MIMO systems," IEEE Trans. Commun., vol. 61, no. 4, pp. 1436-1449, Apr. 2013.

[201] F. Boccardi, R. W. Heath, A. Lozano, T. L. Marzetta, and P. Popovski, "Five disruptive technology directions for 5G," IEEE Commun. Mag., vol. 52, no. 2, pp. 74-80, Feb. 2014.

[202] T. Bai, A. Alkhateeb, and R. W. Heath, Jr., "Coverage and capacity of millimeter-wave cellular networks," IEEE Commun. Mag., vol. 52, no. 9 , pp. 70-77, Sep. 2014

[203] A. L. Swindlehurst, E. Ayanoglu, P. Heydari, and F. Capolino, "Millimeter-wave massive MIMO: The next wireless revolution?" IEEE Commun. Mag., vol. 52, no. 9, pp. 56-62, Sep. 2014.

[204] W. Roh et al., "Millimeter-wave beamforming as an enabling technology for 5G cellular communications: Theoretical feasibility and prototype results," IEEE Commun. Mag., vol. 52, no. 2, pp. 106-113, Feb. 2014

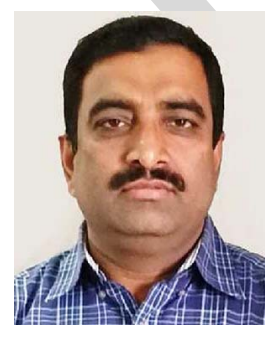

Mohammad Ismat Kadir received the B.Sc.(Eng.) degree (first class) in electrical and electronic engineering and the M.Sc.(Eng.) degree in computer engineering from Bangladesh University of Engineering and Technology, Dhaka, Bangladesh, in 1992 and 1999, respectively, and the Ph.D. degree in electronics and electrical engineering from the University of Southampton, Southampton, U.K., in 2014 .

$\mathrm{He}$ is currently a Faculty Member in the Electronics and Communication Engineering Discipline, Khulna University, Khulna, Bangladesh. His research interests include multicarrier systems, space-time coding, cooperative communications, millimeterwave communications, and heterogeneous networks. Dr. Kadir was a recipient of the Commonwealth Scholarship from the Commonwealth Scholarship Commission, U.K. 


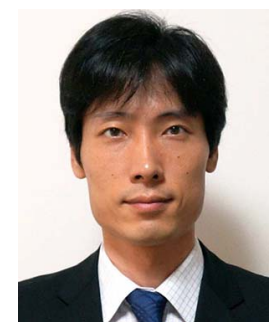

Shinya Sugiura (M'06-SM'12) received the B.S. and M.S. degrees in aeronautics and astronautics from Kyoto University, Kyoto, Japan, in 2002 and 2004, respectively, and the Ph.D. degree in electronics and electrical engineering from the University of Southampton, Southampton, U.K., in 2010.

From 2004 to 2012, he was a Research Scientist with Toyota Central Research and Development Laboratories, Inc., Aichi, Japan. Since January 2013, he has been an Associate Professor with the Department of Computer and Information Sciences, Tokyo University of Agriculture and Technology, Tokyo, Japan, where he heads the Wireless Communications Research Group. He has authored or coauthored over 60 refereed research publications, including 37 IEEE journal and magazine papers. His research has covered a range of areas in wireless communications, networking, signal processing, and antenna design. Dr. Sugiura was the recipient of numerous awards, including the 28th Telecom System Technology Award from the Telecommunications Advancement Foundation in 2013, the 6th IEEE Communications Society Asia-Pacific Outstanding Young Researcher Award in 2011, the 13th Ericsson Young Scientist Award in 2011, and the 2008 IEEE Antennas and Propagation Society Japan Chapter Young Engineer Award. He was also certified as an Exemplary Reviewer of IEEE COMMUNICATIONS LETTERS in 2013 and 2014.

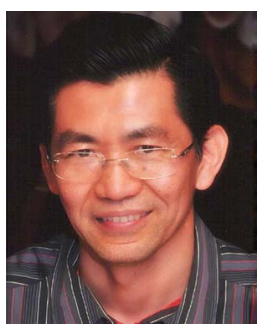

Sheng Chen (M'90-SM'97-F'08) received the B.Eng. degree in control engineering from East China Petroleum Institute, Dongying, China, in 1982, the Ph.D. degree in control engineering from the City University, London, U.K., in 1986, and the D.Sc. degree from the University of Southampton, Southampton, U.K., in 2005.

From 1986 to 1999 , he held research and academic appointments at the Universities of Sheffield, Edinburgh, and Portsmouth, all in U.K. Since 1999, he has been with School of Electronics and Computer Science, University of Southampton, where he currently holds the post of Professor in Intelligent Systems and Signal Processing. He has published over 500 research papers. His research interests include adaptive signal processing, wireless communications, modeling and identification of nonlinear systems, neural network and machine learning, intelligent control system design, evolutionary computation methods, and optimization.

Dr. Chen is a Fellow of the Institute of Engineering and Technology and the U.K. Royal Academy of Engineering. He is a Distinguished Adjunct Professor at King Abdulaziz University, Jeddah, Saudi Arabia, and an ISI Highly Cited Researcher in Engineering (March 2004).

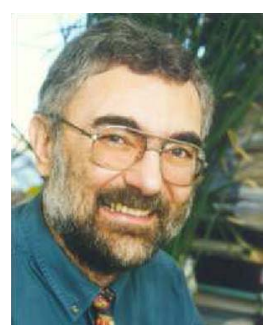

Lajos Hanzo (M'91-SM'92-F'04) received the M.S. degree in electronics and the Ph.D. degree from the Technical University of Budapest, Budapest, Hungary, in 1976 and 1983, respectively; the D.Sc. degree from the University of Southampton, Southampton, U.K., in 2004; and the "Doctor Honoris Causa" degree from the Technical University of Budapest in 2009. During his 38-year career in telecommunications, he has held various research and academic posts in Hungary, Germany, and the U.K. Since 1986, he has been with the School of Electronics and Computer Science, University of Southampton, where he holds the Chair in Telecommunications. He is currently directing a 100-strong academic research team, working on a range of research projects in the field of wireless multimedia communications sponsored by industry, the Engineering and Physical Sciences Research Council U.K., the European Research Council's Advanced Fellow Grant, and the Royal Society's Wolfson Research Merit Award. During 2008-2012, he was a Chaired Professor at Tsinghua University, Beijing. He is an enthusiastic supporter of industrial and academic liaison and offers a range of industrial courses. He has successfully supervised about 100 Ph.D. students, coauthored 20 John Wiley/IEEE Press books on mobile radio communications totaling in excess of 10000 pages, and published more than 1400 research entries at IEEE Xplore. His research is funded by the European Research Council's Senior Research Fellow Grant. Dr. Hanzo is a Fellow of the Royal Academy of Engineering, the Institution of Engineering and Technology, and the European Association for Signal Processing. He is also a Governor of the IEEE Vehicular Technology Society. During 2008-2012, he was the Editor-in-Chief of the IEEE Press. He has served as the Technical Program Committee Chair and the General Chair of IEEE conferences, has presented keynote lectures, and has been awarded a number of distinctions. He has more than 20000 citations. For further information on research in progress and associated publications, please refer to http://www-mobile.ecs.soton.ac.uk. 


\title{
Unified MIMO-Multicarrier Designs: A Space-Time Shift Keying Approach
}

\author{
Mohammad Ismat Kadir, Shinya Sugiura, Senior Member, IEEE, \\ Sheng Chen, Fellow, IEEE, and Lajos Hanzo, Fellow, IEEE
}

\begin{abstract}
A survey and tutorial is provided on the subject of multiple-input-multiple-output (MIMO) multicarrier (MC) systems relying on the space-time shift keying (STSK) concept. We commence with a brief review of the family of MIMO systems, which is followed by the design of STSK systems in the context of MC modulation-based transmissions over dispersive wireless channels. Specifically, the STSK scheme is amalgamated with orthogonal frequency-division multiplexing, MC code-division multiple access, orthogonal frequency-division multiple access (OFDMA), and single-carrier frequency-division multiple access. We also provide a rudimentary introduction to MC differential STSK employing both conventional differential detection and multiple-symbol differential sphere decoding for the sake of dispensing with channel estimation. We conclude with the design recipes of coherent versus noncoherent MC STSK schemes, complemented by a range of future research ideas.
\end{abstract}

Index Terms-Multiple-input multiple-output (MIMO), linear dispersion code (LDC), spatial modulation (SM), space-time shift keying (STSK), orthogonal frequency-division multiplexing (OFDM), multicarrier code-division multiple access (MC-CDMA), orthogonal frequency-division multiple access (OFDMA), singlecarrier frequency-division multiple access (SC-FDMA).

\section{INTRODUCTION}

$\mathbf{M}$ ULTIPLE-input-multiple-output (MIMO) wireless communication systems have attracted substantial research interests over the last two decades, mainly as a benefit of their capability of providing reliable high-throughput communications without extending the bandwidth of a single-antenna system. Taking the explosive expansion of the Internet and the dramatically increased demand for high-speed multimedia wireless systems into consideration, designing flexible and spectrally efficient transceivers is essential. The

Manuscript received January 30, 2014; revised September 19, 2014; accepted November 17, 2014. This work was supported in part by the Commonwealth Scholarship Commission in the U.K., by RC-UK under the auspices of the IUATC, by EU under the CONCERTO project, and by the European Research Council under its Advanced Fellow Grant.

M. I. Kadir was with the School of Electronics and Computer Science, University of Southampton, Southampton SO17 1BJ, U.K. He is now with the Electronics and Communication Engineering Discipline, Khulna University, Khulna 9208, Bangladesh (e-mail: ismat.kadir@ece.ku.ac.bd).

S. Sugiura is with the Department of Computer and Information Sciences, Tokyo University of Agriculture and Technology, Tokyo 184-8588, Japan (e-mail: sugiura@ieee.org).

S. Chen and L. Hanzo are with the School of Electronics and Computer Science, University of Southampton, Southampton SO17 1BJ, U.K. (e-mail: sqc@ecs.soton.ac.uk; 1h@ecs.soton.ac.uk).

Color versions of one or more of the figures in this paper are available online at http://ieeexplore.ieee.org.

Digital Object Identifier 10.1109/COMST.2014.2375321 throughput of a MIMO system may be increased linearly with the number of antennas [1], [2]. Apart from this multiplexing gain, MIMOs are also capable of benefiting from a spatial diversity gain that is attained by the spatially separated antennas in a dense multipath scattering environment, provided that the antenna-elements are sufficiently far apart for the sake of experiencing independent fading. Thus MIMO systems have the potential of improving both the spectral efficiency and the link reliability of future wireless communications systems.

The employment of multiple antenna elements (AEs) may be expected to exhibit flexibility in terms of striking a compelling compromise between the attainable multiplexing versus diversity gains. However, there exists a fundamental limitation, which is quantified in terms of the diversity-multiplexing tradeoff (DMT) [3]. Motivated by this constraint, linear dispersion codes (LDCs) were proposed in [4], [5] for striking a flexible DMT. As their benefit, LDCs constitute a generic family, which subsumes both the family of diversity and multiplexing schemes, whilst potentially outperforming both-albeit, at the cost of a substantially increased decoding complexity. On the other hand, the concepts of spatial modulation (SM) [6] as well as space shift keying (SSK) [7] emerged as a low-complexity design alternative. The SM/SSK schemes adopted the principle of shrewd transmit antenna (TA) activation for attaining an increased bandwidth efficiency. These low-complexity schemes are not, however, capable of attaining beneficial transmit diversity. Motivated by these conflicting design ideas, space-time shift keying (STSK) [8]-[10] was proposed, which extends the concept of pure spatial-domain TA activation of the SM/SSK schemes to both the spatial and temporal dimensions. The STSK system can thus provide substantial diversity- as well as multiplexing gains. However, the majority of STSK studies were focused on non-dispersive, i.e., narrowband propagation scenarios, rather than on realistic wideband scenarios. Against this background, multicarrier (MC) STSK was conceived in [11]-[13] for facilitating STSK based transmissions over dispersive wireless channels. A particularly promising candidate for the next generation fixed and mobile wireless systems is the combination of STSK techniques with the family of MC communications systems.

Realistic wireless channels are dispersive by nature. Dispersive MIMO channels potentially exhibit frequency diversity, provided that the channel's delay-spread is sufficiently high for ensuring that the subcarriers of a MC system fade near-independently. Alternatively, this phenomenon may also be viewed as the independent fading of the channel impulse response (CIR) taps. A MC system effectively converts the 
dispersive wideband channels into a number of low-rate parallel narrowband frequency-flat subchannels. The unmodulated subcarriers are required to maintain a minimum frequency separation in order to remain orthogonal, but the modulated signal spectra corresponding to the different subcarriers may overlap in the frequency-domain (FD). Hence, the available spectrum can be efficiently utilized. Furthermore, MC systems are capable of significantly reducing the receiver's complexity in broadband channels with the aid of using single-tap FD equalization. Thus MIMO systems combined with MC communications appear to be a promising solution for broadband wireless communications systems. Motivated by the flexible DMT provided by the STSK scheme at a low decoding complexity and by the benefits of MIMO-MC configurations, a number of MC STSK schemes were proposed in [11]-[14]. Specifically, STSK schemes employing orthogonal frequency division multiplexing (OFDM) [11], MC code division multiple access (MCCDMA) [12], orthogonal frequency division multiple access (OFDMA) or single-carrier frequency division multiple access (SC-FDMA) [13] were developed.

MIMO systems relying on MC STSK [11], [13] are capable of ensuring link reliability for transmission over realistic dispersive channels, while simultaneously providing a multiplexing gain at a low decoding complexity. However, the AEs of a co-located STSK system typically suffer from spatially correlated large-scale fading imposed by the ubiquitous shadowing effects [15]. For the sake of mitigating this correlation-induced diversity-gain erosion, the concept of cooperative space-time processing [16], [17] has become popular in recent years, because they benefit from the geographically distributed nature of relay nodes (RNs), where the relays may be viewed as the distributed elements of a MIMO system. As a result, each virtual antenna array (VAA) element experiences uncorrelated fading. Additionally, this cooperative MIMO architecture enables us to avoid the physical limitations of installing multiple antennas on a shirt-pocket sized mobile handset. The concept of cooperative STSK [18] was conceived for frequency-flat Rayleigh fading channels in order to benefit from mobile station (MS)cooperation, although naturally, this scheme suffers from the usual throughput loss imposed by the half-duplex relaying strategy employed. The introduction of successive relaying (SR) [19], on the other hand, is potentially capable of recovering the half-duplex multiplexing loss and hence it was successfully used in [20] as a cooperative space-time coding architecture.

Motivated by these insights, a novel cooperative MC STSK arrangement was conceived in [14] for the sake of benefiting from cooperative diversity [21]. In [14], SR was invoked for mitigating the typical $50 \%$ throughput loss of conventional half-duplex relaying schemes, while MC-CDMA is used for circumventing the dispersive effects of wireless channels as well as for reducing the SR-induced interferences. In short, the STSK systems may be classified as shown in Fig. 1. In this tutorial, we will provide design guidelines for OFDM-aided STSK, MC-CDMA aided STSK, OFDMA/SC-FDMA aided STSK, SR aided distributed MC coherent STSK (CSTSK) and differential STSK (DSTSK) using multiple-symbol differential sphere decoding (MSDSD) for communications over dispersive channels.

\section{STSK Systems}

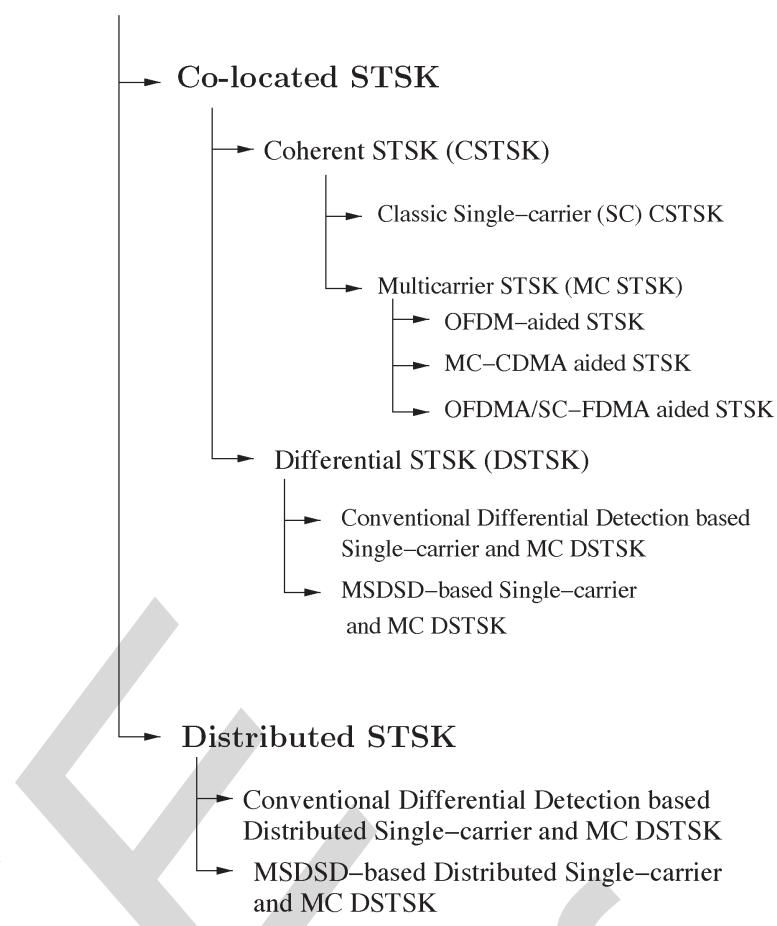

Fig. 1. STSK systems so far studied.

Following a brief review of MIMO systems, of MC systems and of MIMO-MC systems, we will embark on a tutorial on the subject of the family of both co-located and distributed MC STSK systems.

\section{A. Outline}

The remainder of this paper is organized as follows. In Section II, we provide a brief review of the major contributions to the different types of MIMO systems and of the recent design philosophies, which motivated the conception of STSK systems. In Section III, MIMO-OFDM designs are briefly reviewed and the concept of OFDM-aided STSK is described. An overview of multiuser STSK schemes employing MCCDMA and OFDMA/SC-FDMA is provided in Section IV, while distributed MC STSK employing SR is highlighted in Section V. In Section VI, both the hard-decision based maximum likelihood (ML)-MSDSD and the soft-decision based maximum a posteriori (MAP)-MSDSD are invoked for differentially encoded and noncoherently detected OFDM-aided STSK schemes. Section VII provides a range of design guidelines and future research ideas. Finally, Section VIII concludes this treatise.

\section{B. Notations}

The following notations are employed throughout this contribution. Upper-case boldface letter, for example, $\boldsymbol{A}$ denotes a matrix, whereas $\boldsymbol{A}^{T}, \boldsymbol{A}^{H}, \operatorname{tr}(\boldsymbol{A}), \operatorname{vec}(\boldsymbol{A})$, and $\|\boldsymbol{A}\|$ denote the matrix transpose, the Hermitian transpose, the trace, the vectorial stacking operator and the Euclidean norm of $\boldsymbol{A}$ respectively. The operator $|a|$ denotes the complex norm of the complex number $a, \otimes$ the Kronecker product, $\boldsymbol{I}_{T}$ the $(T \times T)$-element 


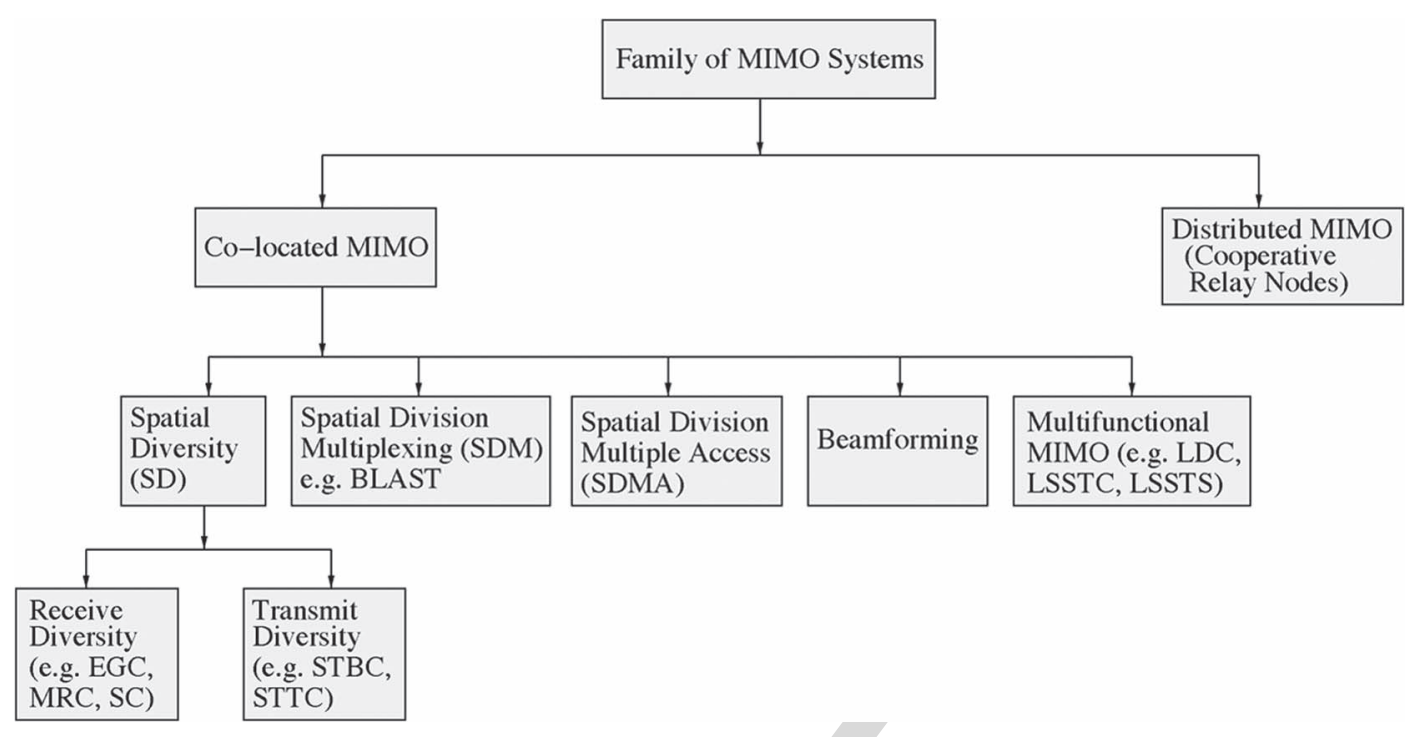

Fig. 2. The simplified family tree of MIMO systems.

identity matrix, $\mathbf{0}_{M \times T}$ the $(M \times T)$-element zero matrix and $\mathfrak{R}(\cdot)$ and $\mathfrak{I}(\cdot)$ the real and the imaginary parts of a complex number, respectively. The notation $\operatorname{diag}\left\{x_{1}, \ldots, x_{N}\right\}$ denotes a diagonal matrix with $x_{1}, \ldots, x_{N}$ on its main diagonal, while $\delta(\cdot)$, $\delta[\cdot]$ and $P(\cdot)$ represent the continuous-time Dirac delta function, the discrete-time Dirac delta function and the probability of ".," respectively. The $N_{c}$-point discrete Fourier transform (DFT) of the symbol stream "•" and the $N_{c}$-point inverse discrete Fourier transform (IDFT) of "•" are denoted by $\mathrm{DFT}_{N_{c}}\{\bullet\}$ and IDFT $_{N_{c}}\{\bullet\}$, respectively. Furthermore, the notation $u$ denotes the generalized user, whereas $v$ refers to the desired user.

\section{MiMO Systems: StATE-OF-THE-ART CONTRIBUTIONS AND STSK}

In this section, we would like to commence with a brief review of the major contributions to the MIMO family, followed by an outline of the recent MIMO techniques, which motivated the invention of the STSK concept.

\section{A. Contributions to Different MIMO Types}

MIMO systems may be classified into different categories [38], [39], as shown in the family tree of Fig. 2, according to the applications they are employed in. The state-of-the-art contributions on different MIMO schemes are summarized in Table I.

Spatial diversity is used in wireless communications systems for combating the deleterious effects of small scale fading and thereby for improving the link's reliability. Diversity combining schemes employed in the receiver, such as equal gain combining (EGC), selection combining (SC) and maximum ratio combining (MRC) can be traced back to the 1950s with reference to Brennan's excellent paper [22]. In order to facilitate diversity-aided transmissions, a simple but witty transmit diversity technique is constituted by Alamouti's scheme [24], which inspired a spate of transmit diversity ideas, as proposed in Tarokh's space-time block code (STBC) and space-time trellis code (STTC) [25], [26].
MIMO systems may be designed for attaining an improved bandwidth efficiency by transmitting different signal streams independently over each of the transmit antennas and are known as spatial division multiplexing (SDM). SDM was first conceived by Paulraj and Kailath [29], who patented the "Distributed Transmit—Directional Receive (DTDR)" concept. Later, Foschini [30] introduced the SDM concept in 1996 under the terminology of Bell Laboratories space-time (BLAST) codes, which later evolved to a modified architecture termed as the vertical BLAST or V-BLAST arrangement proposed by Wolniansky in 1998 [31]. The discovery that for a fixed radiated power and bandwidth the capacity increases linearly with the number of transmit-receive antenna pairs was further supported by Telatar in 1999 [2].

Space-division multiple access (SDMA) employs multiple antennas for the sake of supporting multiple users [34], [40]. Like SDM, SDMA allows us to increase the capacity of a mobile cellular system by exploiting the spatial separation of the users. The spatial gain advantages of SDMA are attained assuming the existence of numerous users having uncorrelated CIRs.

Beamforming architectures are specifically designed for reducing the interference levels with the aid of creating angularly selective beams [33], [41], [42]. Typically, half-wavelengthspaced antenna elements are used for creating a spatially selective transmitter/receiver beam.

There are other types of MIMO systems which are capable of combining the benefits of several MIMO types and may be applied for simultaneously attaining the benefits of having diversity, multiplexing, multiple access and/or beamforming gains. These schemes are referred to as multifunctional MIMO systems [38]. Examples of multifunctional MIMOs include the layered steered space-time codes (LSSTCs) of [37] and the layered steered space-time spreading (LSSTS) of [43].

\section{B. Recent Developments in MIMO Techniques}

In contrast to the classic MIMOs described above, recently a number of MIMO techniques were developed which motivated the STSK concept. Explicitly, the chronological development of 
TABLE I

MAJOR CONTRIBUTIONS CONCEIVED FOR DIFFERENT MIMO APPLICATIONS

\begin{tabular}{|c|c|c|c|}
\hline Category & Year & Author(s) & Contribution \\
\hline \multirow{7}{*}{ Diversity } & 1959 & Brennan [22] & Introduced and analyzed EGC, SC and MRC. \\
\hline & 1994 & $\begin{array}{l}\text { Seshadri and Win- } \\
\text { ters [23] }\end{array}$ & $\begin{array}{l}\text { A transmit diversity scheme was inspired by Wittneben's delay } \\
\text { diversity design. }\end{array}$ \\
\hline & \multirow{3}{*}{1998} & Alamouti [24] & Proposed a transmit diversity scheme relying on two antennas. \\
\hline & & Tarokh et al. [25] & $\begin{array}{l}\text { Conceived space-time block codes based on orthogonal de- } \\
\text { signs. }\end{array}$ \\
\hline & & Tarokh et al. [26] & $\begin{array}{l}\text { Provided design criteria for achieving the maximum attainable } \\
\text { diversity and coding gain. }\end{array}$ \\
\hline & \multirow{2}{*}{2000} & Hughes [27] & Introduced a new DSTBC design based on group codes. \\
\hline & & $\begin{array}{l}\text { Jafarkhani et } \\
\text { al. }[28]\end{array}$ & $\begin{array}{l}\text { Proposed a differential detection scheme for transmit diversity } \\
\text { arrangements. }\end{array}$ \\
\hline \multirow{4}{*}{ Multiplexing } & 1994 & $\begin{array}{ll}\text { Paulraj and } \\
\text { Kailath [29] }\end{array}$ & $\begin{array}{l}\text { Patented the first spatial multiplexing scheme conceived for } \\
\text { broadcast applications. }\end{array}$ \\
\hline & 1996 & Foschini [30] & $\begin{array}{l}\text { Conceived the encoding and decoding of the diagonal BLAST } \\
\text { structure. }\end{array}$ \\
\hline & 1998 & $\begin{array}{l}\text { Wolniansky } \\
\text { al. [31] }\end{array}$ & $\begin{array}{l}\text { The V-BLAST architecture was introduced for reducing com- } \\
\text { plexity in implementing Foscini's approach [30]. }\end{array}$ \\
\hline & 1999 & Telatar [2] & Quantified the MIMO channel capacity. \\
\hline \multirow[t]{2}{*}{ Beamforming } & 1994 & $\begin{array}{ll}\text { Winters } & \text { and } \\
\text { Salz [32] }\end{array}$ & $\begin{array}{l}\text { The impact of diversity of directional transmission on the } \\
\text { capacity was studied. }\end{array}$ \\
\hline & 1997 & Godara [33] & $\begin{array}{l}\text { Antenna arrays were proposed for mobile communications and } \\
\text { their performance was studied. }\end{array}$ \\
\hline \multirow[t]{2}{*}{ SDMA } & 1996 & $\begin{array}{l}\text { Cooper and Gold- } \\
\text { burg [34] }\end{array}$ & Introduced spatial division multiple access. \\
\hline & 2000 & $\begin{array}{l}\text { Vandenameele et } \\
\text { al. [35] }\end{array}$ & Amalgamated OFDM with SDMA. \\
\hline \multirow{4}{*}{ Multifunctional } & \multirow[t]{2}{*}{2002} & $\begin{array}{l}\text { Hassibi and } \\
\text { Hochwald [4] }\end{array}$ & $\begin{array}{l}\text { LDCs were introduced for achieving a flexible diversity- } \\
\text { multiplexing tradeoff. }\end{array}$ \\
\hline & & Heath \& Paulraj [5] & $\begin{array}{l}\text { Improved LDC designs based on frame theoretic matrix lin- } \\
\text { earization were advocated. }\end{array}$ \\
\hline & 2004 & Kim and Chun [36] & $\begin{array}{l}\text { Proposed a MIMO structure, which combines spatial multi- } \\
\text { plexing and beamforming. }\end{array}$ \\
\hline & 2009 & El-Hajjar et al. [37] & Proposed layered steered space-time codes. \\
\hline
\end{tabular}

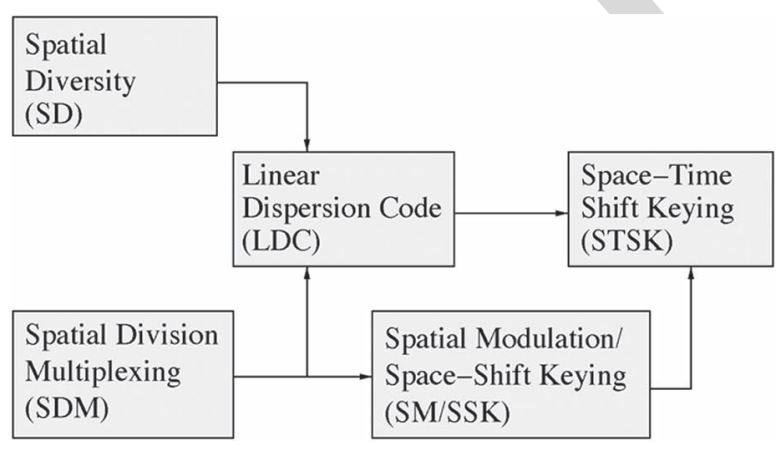

Fig. 3. Development of the STSK concept for striking a flexible DMT.

the MIMO family, which paved the way for the STSK concept is illustrated in Fig. 3 and the major contributions related to SM, SSK and STSK are summarized in Table II.

To be specific, MIMO systems are not expected to be employed for attaining either entirely diversity or solely for multiplexing gain. Rather, in order to strike a flexible DMT
[3], Hassibi and Hochwald [4] proposed LDCs, which were extended by Heath and Paulraj [5] based on frame theory. The flexible benefits of LDC schemes are, however, attained at the cost of imposing high decoding complexity. SM and SSK, on the other hand, are low-complexity solutions which are not capable of providing transmit diversity.

As a predecessor of the SM/SSK/STSK concept, the "space modulation" philosophy which allocates source bits to AEs by simultaneously activating multiple AEs was conceived by Chau and $\mathrm{Yu}$ [44] in 2001. Later, Mesleh et al. [6] introduced the concept of SM for activating only one of the transmit antennas at a time, where the antenna index itself carries additional information, thus attaining an increased throughput. This development was followed by Jeganathan et al. [7], who simply used the presence or absence of energy to transmit information without using any specific signal constellation, which led to the concept of SSK. The SM/SSK [6], [7] scheme employs the antenna index for achieving a multiplexing gain, but owing to activating a single $\mathrm{AE}$, it is incapable of providing a transmit diversity gain. Hence, by intrinsically amalgamating the 
TABLE II

MAJOR CONTRIBUTIONS ON SM/SSK/STSK

\begin{tabular}{|c|c|c|}
\hline Year & Author(s) & Contribution \\
\hline 2001 & Chau and Yu [44] & $\begin{array}{l}\text { Conceived the 'space modulation' philosophy, which allocates source bits to AEs } \\
\text { and simultaneously activates multiple AEs. }\end{array}$ \\
\hline 2006 & Mesleh et al. [45] & $\begin{array}{l}\text { Proposed spatial modulation (SM) for low-complexity spectral efficiency enhance- } \\
\text { ment. }\end{array}$ \\
\hline \multirow{2}{*}{2008} & Mesleh et al. [6] & Presented an analytical investigation of SM. \\
\hline & Mesleh et al. [46] & Analyzed the symbol-error-ratio (SER) of SM. \\
\hline \multirow{2}{*}{2009} & Jeganathan et al. [7] & Conceived space shift keying (SSK). \\
\hline & Jeganathan et al. [47] & $\begin{array}{l}\text { Studied SM as well as SSK and proposed a single-stream optimal ML detection } \\
\text { algorithm. }\end{array}$ \\
\hline \multirow{3}{*}{2010} & Sugiura et al. [8] & Introduced both coherent and differential STSK based on LDCs. \\
\hline & Sugiura et al. [48] & Conceived reduced-complexity detectors for QAM-aided STSK schemes. \\
\hline & Mesleh et al. [49] & Trellis coded SM was introduced. \\
\hline \multirow{8}{*}{2011} & Renzo and Haas [50] & $\begin{array}{l}\text { Studied the performance of SSK-MIMO and proposes a novel SSK-MIMO for } \\
\text { increasing diversity order. }\end{array}$ \\
\hline & Rajashekhar et al. [51] & Proposed a novel DM design for CSTSK based on field extension codes. \\
\hline & Basar et al. [52] & Designed a trellis code for SM. \\
\hline & Yang et al. [53] & $\begin{array}{l}\text { Adaptive SM was proposed by adaptively selecting the modulation order for } \\
\text { improving system performance and for reducing its complexity. }\end{array}$ \\
\hline & Sugiura et al. [9] & Introduced a generalized space-time shift keying arrangement. \\
\hline & Ngo et al. [54] & $\begin{array}{l}\text { Introduced the concept of space-time-frequency shift keying (STFSK) for wideband } \\
\text { channels. }\end{array}$ \\
\hline & Sugiura et al. [18] & Proposed both coherent and noncoherent cooperative STSK. \\
\hline & Renzo et al. [55] & Presented a comprehensive survey of the SM-MIMO scheme. \\
\hline \multirow{8}{*}{2012} & Sugiura et al. $[10]$ & Provided a survey of STSK as a universal MIMO space-time architecture. \\
\hline & $\begin{array}{l}\text { Som and } \\
\text { Chockalingam [56] }\end{array}$ & $\begin{array}{l}\text { Studied SM and SSK in cyclic prefixed single-carrier systems communicating over } \\
\text { dispersive MIMO channels. }\end{array}$ \\
\hline & Basar et al. [57] & $\begin{array}{l}\text { A new class of space-time trellis codes, namely 'super-orthogonal' trellis coded SM } \\
\text { was proposed to achieve maximal diversity and coding gains. }\end{array}$ \\
\hline & Yang et al. [58] & An improved matched-filter detection algorithm was proposed for STSK. \\
\hline & Babich et al. [59] & $\begin{array}{l}\text { Genetic Algorithm based DM optimization was proposed for concatenated coding } \\
\text { assisted STSK. }\end{array}$ \\
\hline & Driusso et al. [11] & Proposed OFDM-aided STSK for dispersive downlink channels. \\
\hline & Rajashekhar et al. [60] & Structured DMs were designed for STSK. \\
\hline & Ngo et al. [61] & $\begin{array}{l}\text { The area spectral efficiency (ASE) of STFSK aided slow-frequency-hopping multiple } \\
\text { access (SFHMA) was investigated. }\end{array}$ \\
\hline \multirow{7}{*}{2013} & Zhang et al. [62] & $\begin{array}{l}\text { A cooperative decode- and forward (DF) DSTSK scheme was proposed employing } \\
\text { MSDSD and multiuser DS-CDMA. }\end{array}$ \\
\hline & Renzo and Haas [63] & Designed SM making provision for achieving transmit diversity gain. \\
\hline & Rajashekhar et al. [64] & Novel TA selection techniques were conceived for SM systems. \\
\hline & $\begin{array}{l}\text { Serafimovski et } \\
\text { al. }[65]\end{array}$ & First practical testbed implementation of SM in the laboratory. \\
\hline & $\begin{array}{ll}\text { Sugiura } & \text { and } \\
\text { Hanzo [66] } & \end{array}$ & Joint DM and constellation optimization was proposed for STSK. \\
\hline & Xu et al. [67] & $\begin{array}{l}\text { Proposed reduced-complexity detection techniques for both SM as well as for STSK } \\
\text { without sacrificing their performance. }\end{array}$ \\
\hline & Kadir et al. [12]-[14] & $\begin{array}{l}\text { Proposed a suite of MC-CDMA- and OFDMA/SC-FDMA-aided co-located and } \\
\text { distributed STSK schemes for dispersive channels. }\end{array}$ \\
\hline \multirow{3}{*}{2014} & Yang et al. [68] & Provided design guidelines for SM-MIMO. \\
\hline & Kadir et al. [69] & Proposed a MC DSTSK scheme employing iterative soft MSDSD. \\
\hline & Renzo et al. [70] & $\begin{array}{l}\text { Presented a review as well as experimental results on SM and discussed its beneficial } \\
\text { application areas. }\end{array}$ \\
\hline
\end{tabular}

concept of LDCs and of SM [6] as well as SSK [7], Sugiura et al. proposed the design philosophy of STSK [8].

1) $S M: \mathrm{SM}$ maps the symbols to the activated transmit antenna index, as an implicit means of conveying extra information. Hence it maps a block of information bits to two information carrying units: to a classic symbol chosen from the constellation and to the unique index of the activated transmit antenna.

The exploitation of the transmit antenna index as an information-bearing unit improves the overall spectral efficiency by $\log _{2} M$ bits, where $M$ denotes the number of transmit antennas. 
The number of bits transmitted using an $\mathcal{L}$-ary constellation equals to $\log _{2} \mathcal{L}$, whereas that using the activated antenna index will be $\log _{2} M$. The SM scheme is capable of transmitting $R$ bits, where $R$ is given by

$$
R=\log _{2}(M \cdot \mathcal{L}),
$$

The most attractive feature of the SM scheme is that it imposes a substantially reduced decoding complexity in comparison to its SDM counterpart.

Since the SM scheme activates a single AE at any signaling interval, only a single radio-frequency (RF) chain is necessitated. Furthermore, the SM scheme is capable of operating using only loose synchronization among the AEs, i.e., it is robust to inter-antenna synchronization (IAS) errors.

2) SSK: In case of SSK aided transmission, the classic PSK/QAM constellation signaling of the SM scheme is deactivated and only the presence or absence of energy is detected at the receiver antenna [7], which facilitates an extremely lowcomplexity decoding.

\section{STSK}

Motivated by the SM/SSK idea of activating a single AE during each symbol durations while mapping source bits to the index of the AE activated, the STSK transmitter activates one out of $Q$ LDC-like dispersion matrices (DMs) $\boldsymbol{A}_{q} \in C^{M \times T}(q=$ $1,2, \ldots, Q)$, which are pre-assigned before transmission. To expound a little further, the DMs or the spreading matrices, as used both in LDC and in STSK, are $(M \times T)$-element matrices of complex numbers, which are generated by optimizing a specific objective function (OF) under a power constraint criterion and are utilized to disperse the source information in both the spatial and time domain [4], [5], [8]. LDCs use a matrix modulation framework [5], where the transmitted codeword is formed by a linear combination of the DMs weighted by the $\mathcal{L}$-PSK/QAM symbols. To be more specific, the LDC transmit codeword $\boldsymbol{X}^{\mathrm{LDC}}[i] \in \mathrm{C}^{M \times T}$ during any signaling interval $i$ corresponding to the symbols $s_{1}[i], s_{2}[i], \ldots, s_{Q}[i]$ and to the DMs

$$
\begin{gathered}
\boldsymbol{A}_{1}=\left[\begin{array}{ccc}
a_{1,1}^{1} & \cdots & a_{1, T}^{1} \\
a_{2,1}^{1} & \cdots & a_{2, T}^{1} \\
\vdots & \ddots & \vdots \\
a_{M, 1}^{1} & \cdots & a_{M, T}^{1}
\end{array}\right], \boldsymbol{A}_{2}=\left[\begin{array}{ccc}
a_{1,1}^{2} & \cdots & a_{1, T}^{2} \\
a_{2,1}^{2} & \cdots & a_{2, T}^{2} \\
\vdots & \ddots & \vdots \\
a_{M, 1}^{2} & \cdots & a_{M, T}^{2}
\end{array}\right], \\
\cdots, \boldsymbol{A}_{Q}=\left[\begin{array}{ccc}
a_{1,1}^{Q} & \cdots & a_{1, T}^{Q} \\
a_{2,1}^{Q} & \cdots & a_{2, T}^{Q} \\
\vdots & \ddots & \vdots \\
a_{M, 1}^{Q} & \cdots & a_{M, T}^{Q}
\end{array}\right]
\end{gathered}
$$

is given by

$$
\begin{aligned}
& \boldsymbol{X}^{\mathrm{LDC}}[i] \\
& =s_{1}[i]\left[\begin{array}{ccc}
a_{1,1}^{1} & \cdots & a_{1, T}^{1} \\
a_{2,1}^{1} & \cdots & a_{2, T}^{1} \\
\vdots & \ddots & \vdots \\
a_{M, 1}^{1} & \cdots & a_{M, T}^{1}
\end{array}\right]+s_{2}[i]\left[\begin{array}{ccc}
a_{1,1}^{2} & \cdots & a_{1, T}^{2} \\
a_{2,1}^{2} & \cdots & a_{2, T}^{2} \\
\vdots & \ddots & \vdots \\
a_{M, 1}^{2} & \cdots & a_{M, T}^{2}
\end{array}\right]
\end{aligned}
$$

$$
+\ldots+s_{Q}[i]\left[\begin{array}{ccc}
a_{1,1}^{Q} & \cdots & a_{1, T}^{Q} \\
a_{2,1}^{Q} & \cdots & a_{2, T}^{Q} \\
\vdots & \ddots & \vdots \\
a_{M, 1}^{Q} & \cdots & a_{M, T}^{Q}
\end{array}\right]
$$

where the DMs are optimized using the power constraint of [5]:

$$
\operatorname{tr}\left(\boldsymbol{A}_{q} \boldsymbol{A}_{q}^{H}\right)=\frac{T}{Q} .
$$

By contrast, the STSK codeword $\boldsymbol{X}^{\text {STSK }}[i]$ transmitted during signaling interval $i$ is generated by activating a single $\mathrm{DM} \boldsymbol{A}_{q}[i]$ in conjunction with the $\mathcal{L}$-PSK/QAM symbol $s_{q}[i]$. Hence, a total of $\log _{2}(Q \cdot \mathcal{L})$ source bits are mapped to each space-time block $\boldsymbol{X}[i] \in \mathcal{C}^{M \times T}$ by the STSK scheme according to [8]

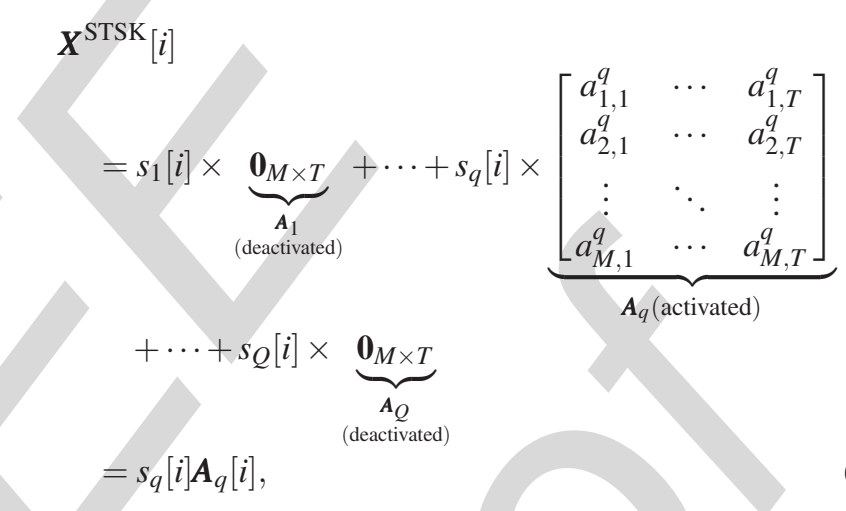

where $\mathbf{0}_{M \times T}$ is an $(M \times T)$-element zero matrix representing the deactivated DM and the $\mathcal{L}$-PSK/QAM symbol $s_{q}[i]$ is represented by $B_{1}=\log _{2} \mathcal{L}$ number of input bits, whereas the specific dispersion matrix $\boldsymbol{A}_{q}[i]$ is selected from the set of $Q \mathrm{DMs}$, $\boldsymbol{A}_{q},(q=1,2, \ldots, Q)$ by the $B_{2}=\log _{2} Q$ number of input bits. Additionally, in order to maintain a unity average transmission power within each STSK symbol duration, each of the DMs has to obey the power constraint of [8]:

$$
\operatorname{tr}\left(\boldsymbol{A}_{q} \boldsymbol{A}_{q}^{H}\right)=T
$$

where $\operatorname{tr}(\bullet)$ indicates the trace of the matrix " $\bullet$."

Since the LDC transmit codeword of (2) incorporates all the $Q$ DMs as well as all the $Q$ constellation symbols, LDCs impose a high decoding complexity [4], [5]. This is due to the presence of different $s_{q} \boldsymbol{A}_{q},(q=1,2, \ldots, Q)$ terms in (2) which introduce interference among themselves during the decoding stage and is referred to as the inter-stream interference [8], [10] in this treatise. The SM and the STSK systems activate a single antenna and a single DM, respectively, at each signaling interval. We refer to this as the single-stream transmission protocol, which does not impose any inter-stream interference [8], [10].

The benefits of the STSK scheme may be summarized as follows [8], [10]:

- The STSK scheme is capable of striking the same DMT as the LDC, but at a substantially reduced complexity. On the other hand, STSK is capable of achieving both transmit 
as well as receive diversity gains, while the SM and SSK schemes attain only receive diversity gain.

- Since only a single one of the $Q$ DMs is activated at any symbol instant, the STSK scheme imposes no interstream interference and - as a further benefit—a singlestream based ML detection can be employed [8].

- STSK is capable of adaptively supporting an arbitrary number of transmit as well as receive AEs.

The normalized throughput per symbol duration of the STSK scheme may be expressed as [8]

$$
R=\frac{\log _{2}(\mathcal{L} \cdot Q)}{T} \quad\left[\frac{\text { bit }}{\text { symbol duration }}\right] .
$$

Furthermore, the STSK system is uniquely and unambiguously specified by the parameters $(M, N, T, Q)$ in conjunction with the classic $\mathcal{L}$-PSK or $\mathcal{L}$-QAM modulation.

1) DM Optimization for STSK: The performance of the STSK system substantially depends on the choice of the DMs. More specifically, it depends firstly on the specific OF utilized and secondly on the DM optimization procedure. The optimization of the set of DMs is thus of utmost importance for the performance of the STSK scheme.

a) Random search based DM optimization: In the STSK encoder described by (4), $Q$ DMs, $\boldsymbol{A}_{q}(q=1, \ldots, Q)$ are preassigned to the transmitter in advance of transmission, which may be generated by searching exhaustively through a large set of randomly generated matrices and selecting the best ones using some optimization criterion as mentioned in [5] and detailed further in [38]. More specifically, the search process consists of generating a set of for example $10^{6} \mathrm{DMs}$, which have entries distributed according to the complex Gaussian distribution $\operatorname{e\mathcal {N}}(0,1)$ and which are normalized in order to satisfy the power-constraint criterion of (5) and then picking the best $Q$ matrices, $\boldsymbol{A}_{q}(q=1, \ldots, Q)$. More explicitly, the "quality" of the set generated is evaluated in terms of an OF, such as for example, the corresponding continuous input continuous output memoryless channel (CCMC) capacity [38], the discrete input continuous output memoryless channel (DCMC) capacity [71] and the pairwise error probability (PEP) [5] in terms of the rank and/or determinant criterion of [5], [26] etc.

b) Genetic algorithm aided DM optimization: In addition to the random search-based DM optimization mentioned above, genetic algorithm (GA) may also be employed for reducing the computational burden by searching through only a fraction of the DMs, yet finding the best ones with a high probability [59], [72]. The flowchart of the GA process for the optimization of the STSK spreading matrices is portrayed in Fig. 4. GAs [73] constitute adaptive heuristic search algorithms, where a population of individuals follows the evolutionary concepts of natural selection and genetic operators of crossover and mutation to optimize the OF or fitness function. In the DM optimization problem, an individual is defined as a set of DMs, which will be used for constructing the STSK scheme under the power constraint of (5). The initial population of DMs is randomly selected from a complex-valued Gaussian distribution of zero mean and unity variance. Thereafter the algorithm proceeds recursively. Each recursive stage is termed as a generation and the process is continued up to a pre-specified number of generations.

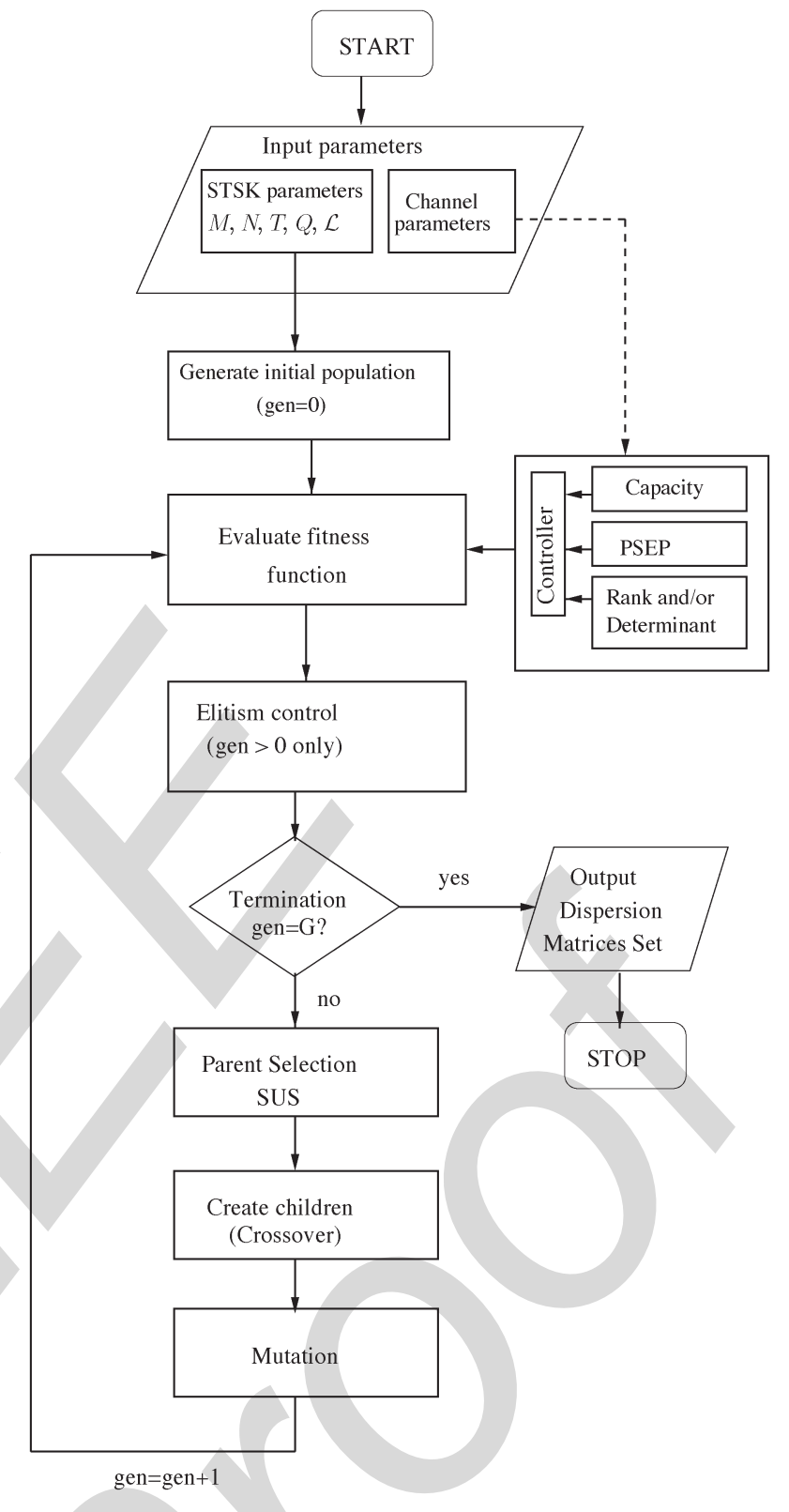

Fig. 4. Generation of the GA-optimized dispersion matrix set to be used in STSK.

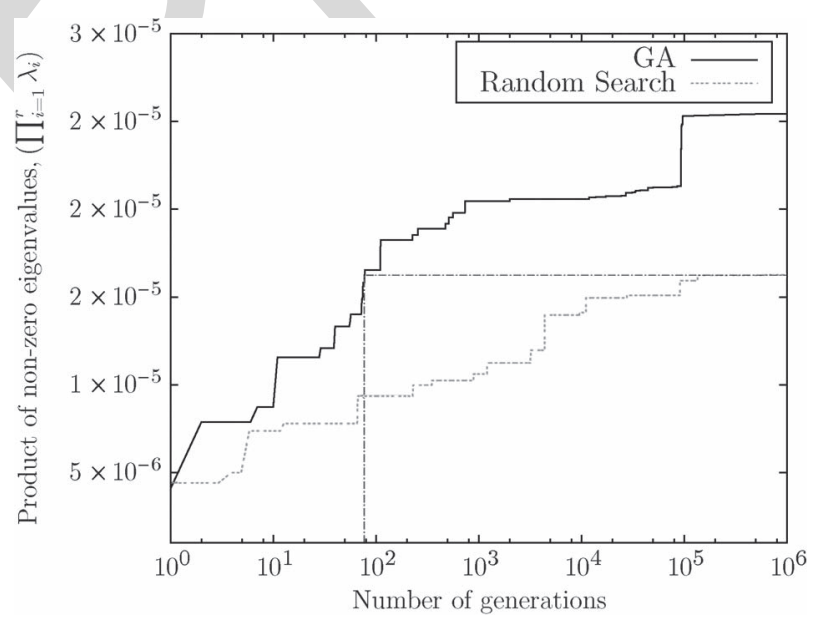

Fig. 5. Fitness function versus the number of generations in both GA optimization and in random search. 


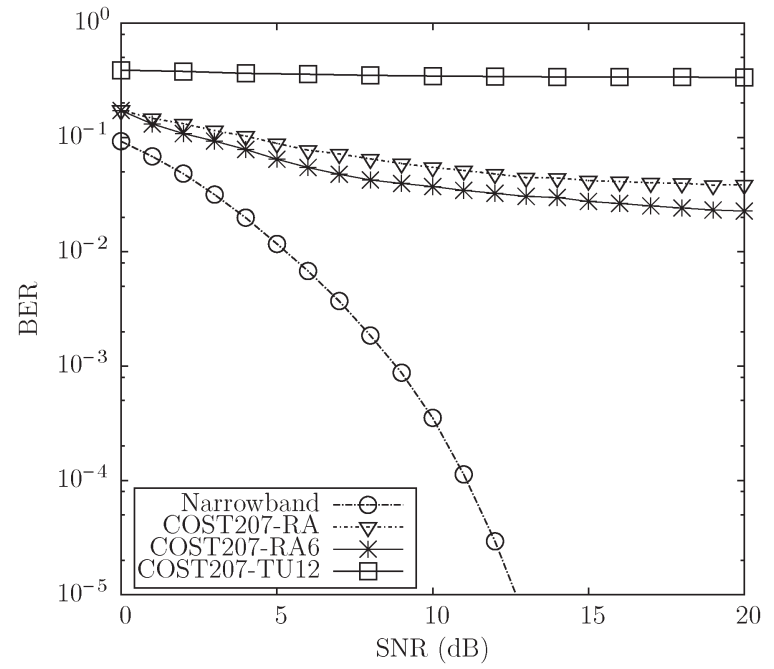

Fig. 6. BER performance of STSK $(2,2,2,2)$ scheme in conjunction with BPSK modulation using the optimal ML detector of [8] under different channel conditions. Although STSK performs well in non-dispersive channels, its performance in multipath channels becomes poor [12]. The system parameters are listed in Table III.

The performance of the GA-based DM optimization can be visualized in Fig. 5. Observe that for the fitness function as defined by the determinant criterion of [26], we approach the same fitness value at about 80 generations which were only approached by the random search after $10^{6}$ iterations. This clearly demonstrates the efficacy of the GA based optimization of DMs. Further insights on the optimization of the DMs for the STSK system may be found in [51], [60], [74].

2) Wideband Channels and the Performance of STSK in Different Channels: A time-invariant broadband channel has an impulse response, which can be modelled as [75], [76]:

$$
h(t, \tau)=\sum_{l=0}^{(L-1)} a_{l} g_{l}(t) \delta\left(\tau-\tau_{l}\right),
$$

where $L$ is the number of multipath components in the channel, $a_{l}, \tau_{l}$, and $g_{l}(t)$ are the channel's envelope, delay and Rayleighdistributed magnitude fading process exhibiting a particular normalized Doppler frequency $f_{d}$ respectively, associated with the $l$-th path, while $\delta($.$) is the Dirac Delta function. In fact,$ the wideband propagation channel is constituted by the superposition of a number of dispersive fading paths, suffering from various attenuations and delays, aggravated by the Doppler shift as a result of the mobile station (MS)'s movement.

The single-carrier STSK is characterized both in narrowband and wideband environments in Fig. 6. The basic simulation parameters are listed in Table III. It is demonstrated in Fig. 6 that the single-carrier STSK $(2,2,2,2)$ scheme operating in conjunction with BPSK modulation works well in narrowband scenarios, but it exhibits a severe error floor in dispersive channels, when the COST207-RA [77] channel model is considered. In the more dispersive typical urban (TU) scenario [75], [77], the STSK scheme suffers from a substantial further performance degradation. The simulation results characterizing STSK for different channel situations are shown in Fig. 6. Observe that the STSK scheme's bit-error ratio (BER) performance is better
TABLE III

Main System Parameters for STSK In DifFERENT ChanNels

\begin{tabular}{|l|r|}
\hline Simulation parameter & Values \\
\hline Fast fading model & 0.01 \\
Doppler frequency & Correlated Rayleigh fading \\
Channel specification & Narrowband channel \\
No. of OFDM subcarriers & 64 \\
Length of cyclic prefix & 32 \\
No. of Tx AE, $M$ & 2 \\
No. of Rx AE, $N$ & 2 \\
No. of Tx time slots, $T$ & 2 \\
No. of dispersion matrices & $Q=2,4$ \\
STSK specification & $\mathcal{L}=2,4$ \\
Modulation order & $(2,2,2, Q), Q=2,4$ \\
\hline
\end{tabular}

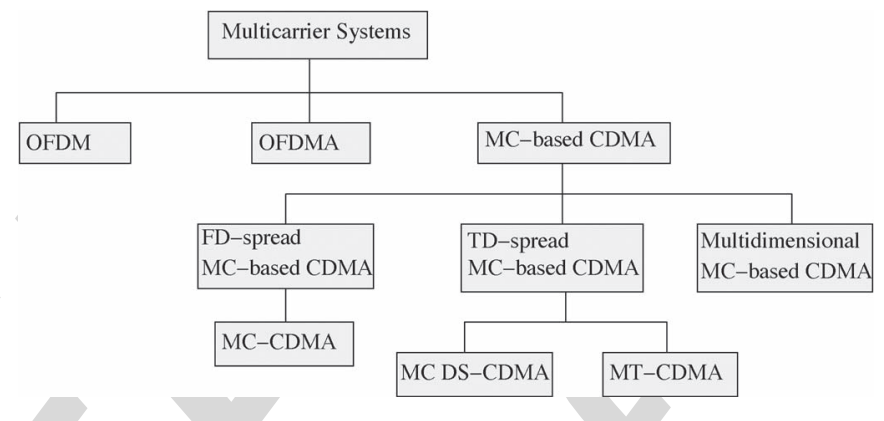

Fig. 7. Simplified family-tree of multicarrier systems.

for transmission over the COST207-RA6 channel model than that over the COST207-RA model, although the number of channel taps is higher in the COST207-RA6 model. This is because although the COST207-RA model has less taps, it has a higher total delay spread than the COST207-RA6 channel model.

The eroded performance of the single-carrier STSK scheme constituted the motivation for conceiving MC based STSK systems. A suite of MC systems, such as OFDM, MC-CDMA, OFDMA and/or SC-FDMA combined both with coherently and differentially detected STSK, was conceived. A range of coherent and noncoherent cooperative MC-CDMA STSK schemes employing SR for recovering the half-duplex throughput loss of conventional relaying schemes was also conceived. Let us now have a discourse on MC-aided STSK systems.

\section{MIMO-MC DESIGNS: EXAMPLE OF THE OFDM-AIDED STSK}

The simplified family-tree of MC systems is shown in Fig. 7. The most widespread family is constituted by OFDM [78]. The combination of OFDM with MIMO systems has attracted substantial interests due to OFDM's capability of converting realistic wideband channels into a number of low-rate, nondispersive subchannels [79]-[81]. Furthermore, OFDM facilitates the employment of single-tap FD equalizers at the receiver, which is highly beneficial in the context of MIMO systems.

\section{A. OFDM and MIMO-OFDM}

OFDM was ratified by numerous standardization bodies, such as the European Digital Audio Broadcasting (DAB) [82] 
TABLE IV

MAJOR CONTRIBUTIONS ON OFDM

\begin{tabular}{|c|c|c|}
\hline Year & Author(s) & Contribution \\
\hline 1966 & Chang [86] & The concept of OFDM was proposed for dispersive channels. \\
\hline 1970 & Chang [91] & U.S. Patent $3,488,445$ on OFDM was issued. \\
\hline 1971 & $\begin{array}{l}\text { Weinstein and } \\
\text { Ebert [87] }\end{array}$ & DFT was first applied for OFDM modulation. \\
\hline 1985 & Cimini $[88]$ & The feasibility of OFDM in mobile communications was studied. \\
\hline 1987 & $\begin{array}{l}\text { Alard and Lasalle } \\
\text { [89] }\end{array}$ & Proposed OFDM for digital broadcasting. \\
\hline 1991 & Cioffi [92] & Introduced the ANSI ADSL standard. \\
\hline 1999 & & IEEE 802.11a standard \\
\hline 2000 & $\begin{array}{lcr}\text { V. Nee and } \\
\text { Prasad }[93]\end{array}$ & First book on OFDM. \\
\hline 2003 & Hanzo et al. [94] & $\begin{array}{l}\text { Design-recipes and analytical insights were detailed in the context of broad- } \\
\text { band multiuser communications, WLANs and broadcasting. }\end{array}$ \\
\hline 2005 & Williams et al. [95] & A pre-FFT synchronisation method was designed for OFDM. \\
\hline 2009 & Chen et al. [96] & Spectrum sensing of pilot tone based OFDM systems was studied. \\
\hline 2014 & Sahin et al. [97] & $\begin{array}{l}\text { Multicarrier schemes are evaluated from the perspective of practical imple- } \\
\text { mentation aspects. }\end{array}$ \\
\hline
\end{tabular}

TABLE V

MAJOR CONTRIBUTIONS ON MIMO-OFDM

\begin{tabular}{|c|c|c|}
\hline Year & Author(s) & Contribution \\
\hline \multirow{2}{*}{2001} & Blum et al. $[90]$ & Improved space-time coding was proposed for MIMO-OFDM . \\
\hline & $\begin{array}{l}\text { Piechocki et } \\
\text { al. [98] }\end{array}$ & Studied the performance of ML decoding-aided V-BLAST OFDM. \\
\hline \multirow{3}{*}{2002} & Bolcskei et al. [99] & $\begin{array}{l}\text { Both the ergodic capacity and the outage capacity of spatial multiplexing aided } \\
\text { MIMO OFDM were determined. }\end{array}$ \\
\hline & Li et al. [100] & $\begin{array}{l}\text { Space-time coding based MIMO OFDM was proposed for mitigating the ISI } \\
\text { and for enhancing the system's capacity. }\end{array}$ \\
\hline & $\begin{array}{l}\text { Stamoulis } \\
\text { al. }[101]\end{array}$ & $\begin{array}{l}\text { Studied STBC based MIMO OFDM for transmission over time-varying } \\
\text { channels. }\end{array}$ \\
\hline \multirow[t]{2}{*}{2003} & $\begin{array}{l}\text { El-Gamal } \\
\text { al. }[102]\end{array}$ & $\begin{array}{l}\text { Employed OFDM for frequency-selective MIMO channels to construct space- } \\
\text { frequency codes (SFC) for exploiting the FD diversity. }\end{array}$ \\
\hline & Moon et al. [103] & $\begin{array}{l}\text { Proposed a peak-to-average power ratio (PAPR) control scheme for MIMO- } \\
\text { OFDM. }\end{array}$ \\
\hline \multirow[t]{2}{*}{2004} & Stuber et al. [79] & $\begin{array}{l}\text { Summarized the physical layer research challenges of MIMO-OFDM wireless } \\
\text { systems. }\end{array}$ \\
\hline & $\begin{array}{l}\text { Huang } \\
\text { Letaief [104] }\end{array}$ & $\begin{array}{l}\text { A coded OFDM-aided MIMO symbol-based space diversity scheme was } \\
\text { conceived. }\end{array}$ \\
\hline \multirow[t]{2}{*}{2005} & Su et al. $[105]$ & $\begin{array}{l}\text { Proposed a full-rate, full-diversity OFDM-aided space-frequency code for } \\
\text { MIMO assisted wireless systems. }\end{array}$ \\
\hline & $\begin{array}{l}\text { Borgmann and } \\
\text { Bolcskei [106] }\end{array}$ & $\begin{array}{l}\text { Non-coherent space-frequency coded OFDM was proposed for dispersive } \\
\text { MIMO channels. }\end{array}$ \\
\hline 2006 & $\begin{array}{ll}\text { Liew } & \text { and } \\
\text { Hanzo [107] } & \end{array}$ & $\begin{array}{l}\text { Investigated space-time block and space-time trellis codes versus adaptive } \\
\text { modulation and coding (AMC) aided OFDM. }\end{array}$ \\
\hline 2007 & $\begin{array}{l}\text { Jiang } \\
\text { Hanzo [40] }\end{array}$ & $\begin{array}{l}\text { Reduced-complexity genetic algorithm assisted multiuser MIMO-OFDM was } \\
\text { investigated. }\end{array}$ \\
\hline 2013 & $\begin{array}{l}\text { Miridakis and Ver- } \\
\text { gados [108] }\end{array}$ & $\begin{array}{l}\text { Reviewed successive interference cancellation schemes in the context of } \\
\text { single-antenna and multiple-antenna OFDM systems. }\end{array}$ \\
\hline 2014 & $\begin{array}{l}\text { Al-Dweik et } \\
\text { al. }[109]\end{array}$ & $\begin{array}{l}\text { A novel technique was proposed to enhance the robustness of conventional } \\
\text { MIMO-OFDM systems. }\end{array}$ \\
\hline
\end{tabular}

and the Digital Video Broadcasting (DVB) [83] standards and Local Area Mobile Wireless Networks, such as the IEEE 802.11a [84] and HIPERLAN/2 [85].

The major contributions on OFDM and MIMO-OFDM are summarized in Table IV and Table V, respectively. The first
OFDM scheme was proposed by Chang [86] in 1966 for dispersive channels. Weinstein et al. [87] demonstrated that OFDM modulation/demodulation can be efficiently performed using the DFT, which was a driving force behind the development of the OFDM scheme. In 1985, Cimini [88] proposed OFDM 


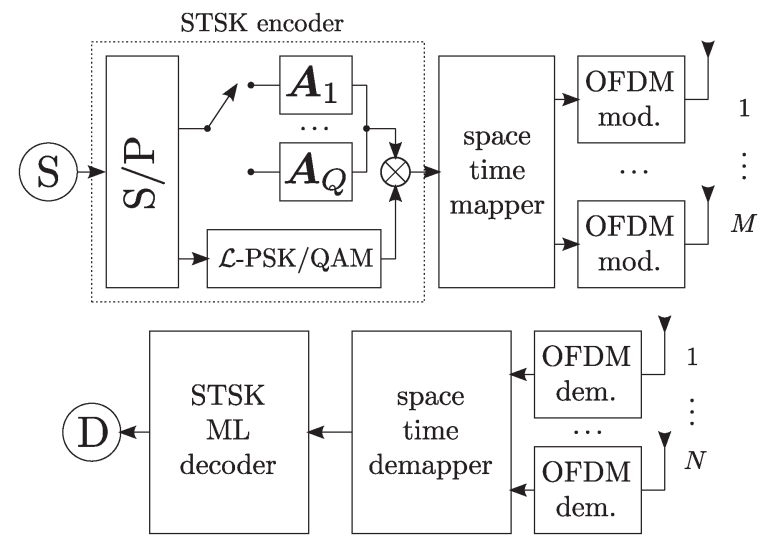

Fig. 8. The transceiver architecture of the OFDM-aided STSK system [11].

for mobile communications, whereas its application for digital broadcasting was proposed by Alard and Lassale [89] in 1985 and 1987, respectively.

In 2001, Blum et al. [90] proposed an improved space-time coding arrangement for MIMO-OFDM wireless systems. As a further advance, Stuber et al. [79] summarized the physical layer research challenges of MIMO-OFDM.

In the spirit of the above-mentioned MIMO-OFDM schemes, a range of MC STSK systems were developed for either single-user or multiuser communications over dispersive channels [11]-[14]. Before proceeding to the multiuser MC STSK schemes, let us first focus on OFDM-aided STSK scheme which was conceived for single-user systems. In [54], the authors proposed space-time-frequency shift keying (STFSK) as a technique capable of exploiting space-, time- and frequency diversity. Nevertheless, this realization of the STFSK scheme suffers from the typical drawbacks of non-coherent FSK demodulation. These impediments of STFSK may be eliminated by reduced-rate OFDM-style parallel transmissions over numerous non-dispersive sub-carriers.

\section{B. OFDM-Aided STSK}

OFDM-aided STSK [11] was conceived for mitigating the performance erosion of STSK schemes operating in wideband channels as demonstrated in Fig. 6 of Section II-C2. The basic idea of the OFDM-aided STSK system is to use the OFDM technique in order to create a number of parallel FD subchannels, where the sub-channel bitrate is sufficiently low for the sub-channel symbols to avoid dispersion. The model of [79] has been adopted for realizing an STSK-based MIMO-OFDM system obeying the system's architecture of Fig. 8. Having generated the STSK blocks, the blocks of symbols are then mapped to $N_{\mathrm{c}}$ subcarriers. As shown in Fig. 9, $N_{\mathrm{c}}$ STSK codewords are arranged in parallel, which constitute an OFDM-STSK frame. Furthermore, OFDM modulation is carried out over the shaded $N_{c}$-length symbol-pipe at AE $m(m=1, \ldots, M)$ during time slot $T_{i}\left(T_{i}=1, \ldots, T\right)$, which represents an OFDM symbol. The time-domain (TD) STSK-OFDM signal transmitted from the $m$ th transmit $\mathrm{AE}$ at time slot $T_{i}$ may be formulated as [94], [110], [111]:
Transmit AE index, $m$

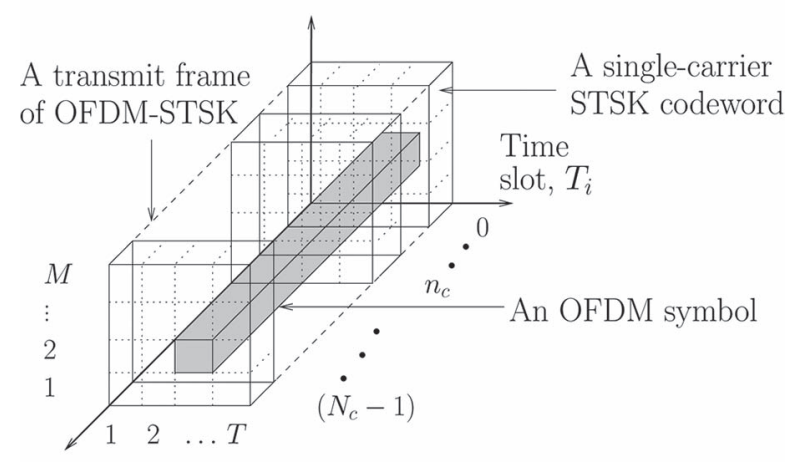

Subcarrier, $n_{c}$

Fig. 9. Formation of an STSK-OFDM frame consisting of $N_{c}$ parallel STSK blocks to be transmitted over $N_{c}$ parallel subcarriers. The shaded symbol-pipe shown represents one of the $(M \cdot T)$ OFDM symbols [69].

$$
x_{m, T_{i}}(t)=\frac{1}{\sqrt{N_{c}}} \sum_{n_{c}=0}^{\left(N_{c}-1\right)} X_{m, T_{i}}\left[n_{c}\right] e^{j 2 \pi f_{n_{c}} t}, \quad 0 \leq t \leq N_{c} \mathcal{T}
$$

where $X_{m, T_{i}}\left[n_{c}\right]$ is the $n_{c}$-th data symbol, while $N_{c} \mathcal{T}$ is the length of the OFDM symbol. In order to maintain the orthogonality of the subcarriers, the subcarrier frequencies have to be equally spaced by $f_{n_{c}}=\frac{n_{c}}{N_{\mathcal{C}} \mathcal{T}}$ and the TD sampling of the OFDM symbol can be formulated as:

$$
\begin{aligned}
x_{m, T_{i}}\left(n_{s} \mathcal{T}\right)=\frac{1}{\sqrt{N_{c}}} \sum_{n_{c}=0}^{\left(N_{c}-1\right)} X_{m, T_{i}}\left[n_{c}\right] e^{j 2 \pi \frac{n_{s} n_{c}}{N_{c}}}, \\
0 \leq n_{s} \leq\left(N_{c}-1\right)
\end{aligned}
$$

which is expressed in terms of the $N_{c}$-point IDFT of the symbol stream $\boldsymbol{X}_{m, T_{i}}=\left\{X_{m, T_{i}}\left[n_{c}\right]\right\}_{n_{c}=0}^{\left(N_{c}-1\right)}$ as:

$$
\boldsymbol{x}_{m, T_{i}}=\operatorname{IDFT}_{N_{c}}\left\{\boldsymbol{X}_{m, T_{i}}\right\}
$$

where $m=1, \ldots, M$ and $T_{i}=1, \ldots, T$. The usual CP, which has to be longer than the channel's delay spread, is appended to each of the TD OFDM symbols in order to eliminate the effects of ISI.

Let the signals from the transmit AEs be transmitted over a frequency-selective channel, where the discrete-time CIR may be modeled as:

$$
\boldsymbol{H}\left[n_{c}\right]=\sum_{l=0}^{L-1} \boldsymbol{H}[l] \delta\left[n_{c}-l\right] \in \mathbb{C}^{N \times M},
$$

where the matrix $\boldsymbol{H}[l] \in \mathbb{C}^{N \times M}$ has complex-valued normally distributed entries according to $\mathcal{E N}\left(\mu_{l}, \sigma_{l}^{2}\right)$ for every channel path $l=0, \ldots,(L-1)$ and $\delta[\cdot]$ is the discrete-time Dirac delta function. After applying the $N_{c}$-point DFT operation at each receive $\mathrm{AE}$ and defining the FD channel transfer matrix $\tilde{\boldsymbol{H}}_{n, m}=\operatorname{diag}\left\{\tilde{h}_{n, m}[0], \tilde{h}_{n, m}[1], \ldots, \tilde{h}_{n, m}\left[N_{c}-1\right]\right\} \in \mathbb{C}^{N_{c} \times N_{c}}$ as a diagonal matrix [13], [94] whose diagonal elements are $\tilde{\boldsymbol{h}}_{n, m}=$ $\operatorname{DFT}_{N_{c}}\left\{\boldsymbol{h}_{n, m}\right\}$, the FD channel-output matrix $\boldsymbol{Y}$ at the receiver may be expressed by 


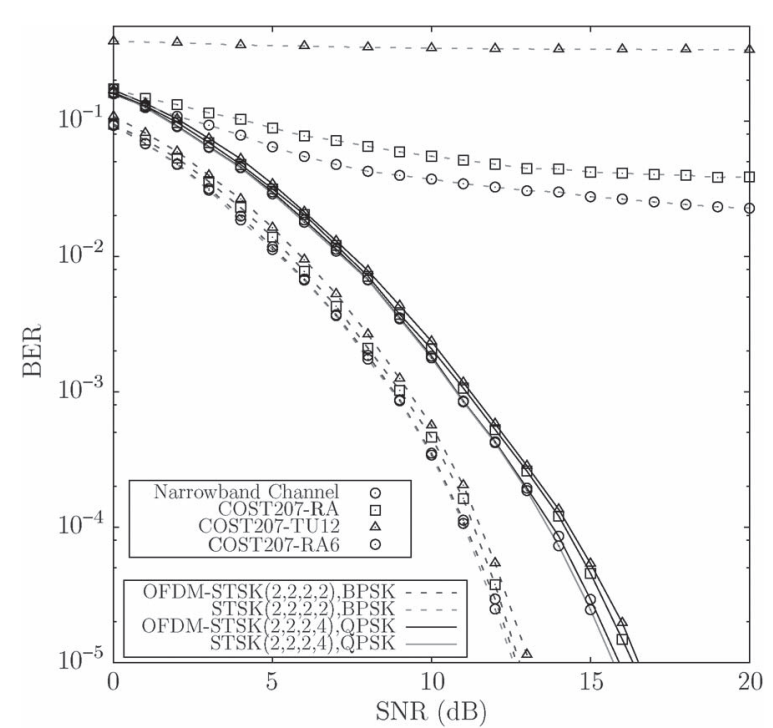

Fig. 10. Performance of the OFDM-aided STSK system of Fig. 8 in dispersive channels. No channel-coding was used and the optimal ML detector of [8] was employed. OFDM-aided STSK exhibits a performance in dispersive channels, which is similar to that in nondispersive environments.

$$
\boldsymbol{Y}\left[n_{c}\right]=\tilde{\boldsymbol{H}}\left[n_{c}\right] \boldsymbol{X}\left[n_{c}\right]+\boldsymbol{V}\left[n_{c}\right], \quad n_{c}=0, \ldots,\left(N_{c}-1\right)
$$

where $\boldsymbol{Y}\left[n_{c}\right] \in \mathbb{C}^{N \times T}, \tilde{\boldsymbol{H}}\left[n_{c}\right] \in \mathbb{C}^{N \times M}, \boldsymbol{X}\left[n_{c}\right] \in \mathbb{C}^{M \times T}$, and $\boldsymbol{V}\left[n_{c}\right] \in$ $\mathbb{C}^{N \times T}$. Following the OFDM demodulation at the space-time demapper, the received symbols $\boldsymbol{Y}\left[n_{c}\right] \in \mathbb{C}^{N \times T}$ are then passed through the single-stream ML decoder [8]. The singlestream based ML estimates $\left(\hat{l}_{c}, \hat{q}\right)$ of the $n_{c}$-th STSK-OFDM symbol and its constituent transmitted bits are obtained from [7], [8] as:

$$
\left(\hat{l}_{c}\left[n_{c}\right], \hat{q}\left[n_{c}\right]\right)=\underset{l_{c}, q}{\arg \min }\left\{\left\|\overline{\boldsymbol{Y}}\left[n_{c}\right]-\overline{\tilde{\boldsymbol{H}}}\left[n_{c}\right] \boldsymbol{\chi} \boldsymbol{K}_{l_{c}, q}\right\|^{2}\right\}
$$

where

$$
\begin{aligned}
\overline{\boldsymbol{Y}}\left[n_{c}\right] & =\operatorname{vec}\left(\boldsymbol{Y}\left[n_{c}\right]\right) \in \mathbb{C}^{N T \times 1}, \\
\overline{\tilde{\boldsymbol{H}}}\left[n_{c}\right] & =\boldsymbol{I}_{T} \otimes \tilde{\boldsymbol{H}}\left[n_{c}\right] \in \mathbb{C}^{N T \times M T}, \\
\boldsymbol{\chi} & =\left[\operatorname{vec}\left(\boldsymbol{A}_{1}\right), \cdots, \operatorname{vec}\left(\boldsymbol{A}_{Q}\right)\right] \in \mathbb{C}^{M T \times Q},
\end{aligned}
$$

and

$$
\boldsymbol{K}_{l_{c}, q}=[\underbrace{0, \cdots, 0}_{q-1}, s_{l_{c}}, \underbrace{0, \cdots, 0}_{Q-q}]^{T} \in \mathbb{C}^{Q \times 1} .
$$

The performance degradation of the single-carrier STSK system in wideband channels was quantified in Section II-C, which was substantially mitigated by the OFDM-aided STSK system of Fig. 8, as portrayed in Fig. 10. Observe from Fig. 10 that the OFDM-aided STSK performance in wideband channels essentially remains unaffected by the channel's dispersion. We see from Fig. 10 that if the channel is non-dispersive, there is virtually no difference in the performance of STSK, regardless whether OFDM has been incorporated into the system or not. In such channel conditions, both the STSK and the OFDM- aided STSK are capable of attaining excellent diversity versus multiplexing gain tradeoffs. By contrast, under dispersive channel conditions, OFDM-aided STSK is capable of maintaining its superiority not only in the rural scenario of the COST207RA channel model, but also in the typical urban scenario modeled by the COST207-TU12 channel model. In fact, the OFDM-aided STSK performance recorded in different channel situations remains more or less similar to that of the narrowband benchmarker.

\section{MC STSK SCHEMES FOR DISPERSIVE MULTIUSER SCENARIOS}

In this section, we provide a brief review of MC-based CDMA, OFDMA and SC-FDMA systems, which is followed by an overview of the amalgamated MC-CDMA aided STSK [12], [112] and OFDMA-assisted and SC-FDMA-aided STSK schemes for supporting multiuser transmissions over dispersive wireless channels. A reduced-complexity detector designed for the OFDMA/SC-FDMA-aided STSK scheme is also discussed.

\section{A. MC-Based CDMA, OFDMA, and SC-FDMA Systems}

The major contributions on MC-CDMA, SC-FDMA and MIMO SC-FDMA are summarized in Table VI. Historically speaking, in 1993, Fettweis et al. developed the MC-CDMA scheme of [113]. Chouly et al. [114] as well as DaSilva and Sousa developed the MC DS-CDMA scheme of [115], whereas the so-called multitone (MT)-CDMA scheme was developed by Vandendorpe [116], all in the same year. In 1997, Hara and Prasad [117] provided a well-cited overview of MC based CDMA systems.

MC-CDMA is a multiple access scheme, where the symbols of different users are first spread across the FD using unique, user-specific spreading sequences, which are then mapped to multiple parallel subcarriers [94], [117]. In the MC DS-CDMA scheme, the data stream is first divided into a number of parallel substreams, each substream is then spread across the TD with the aid of the spreading sequences and then transmitted over one of the subcarriers [117], [118]. The MT-CDMA scheme [116] relies on similar operations to those of MC DS-CDMA, except that the subcarriers are orthogonal to each other before spreading, but the spectrum of subcarriers no longer exhibits orthogonality after TD-spreading. There have also been substantial developments in the context of multidimensional spreading assisted MC-based CDMA schemes [119]-[121], which spread the user information across both the spatial-as well as time-domains or even over the spatial domain (SD). OFDMA [122] is a multiple access version of OFDM, where a block of subcarriers is allocated to the different users in a frequency-division multiple access fashion.

In the aftermath of widespread MC applications, singlecarrier modulation (SCM) is enjoying a renaissance, especially in the context of UL scenarios [123]. Traditionally equalization has been performed in the TD for single carrier transmissions. However, if the ISI spreads over more than for example 30-50 symbols, the signal processing complexity associated with this TD approach might become excessive [123]. As an alternative, FD equalization (FDE) was first studied in [124]. 
TABLE VI

MAJOR CONTRIBUTIONS ON MC-CDMA AND SC-FDMA

\begin{tabular}{|c|c|c|}
\hline Year & Author(s) & Contribution \\
\hline 1973 & $\begin{array}{l}\text { Walzman and Schwartz } \\
{[124]}\end{array}$ & Introduced frequency domain equalization (FDE). \\
\hline \multirow{4}{*}{1993} & Yee et al. [113] & $\begin{array}{l}\text { The concept of FD MC-CDMA combining FD-spreading and OFDM was } \\
\text { introduced. }\end{array}$ \\
\hline & Chouly et al. [114] & Combined multicarrier techniques to DS-CDMA. \\
\hline & DaSilva \& Sousa [115] & Developed MC DS-CDMA. \\
\hline & Vandendorpe [116] & Introduced the concept of multitone DS-CDMA. \\
\hline 1995 & Sari et al. [125] & Proposed SC-FDE for digital terrestrial TV broadcasting. \\
\hline 1997 & Hara and Prasad [117] & Provided an overview of MC-CDMA systems. \\
\hline 1998 & Sorger et al. [130] & $\begin{array}{l}\text { Introduced IFDMA applicable for both the uplink and the downlink of a } \\
\text { mobile radio communications system. }\end{array}$ \\
\hline \multirow{2}{*}{2002} & Falconer et al. [126] & SC-FDE was reviewed and compared to OFDM. \\
\hline & $\begin{array}{l}\text { Benvenuto and } \\
\text { Tomasin [131] }\end{array}$ & Proposed a new FD decision feedback equalizer (DFE) for SC-FDE. \\
\hline 2003 & Hanzo et al. [94] & $\begin{array}{l}\text { The state-of-the-art in OFDM and MC-CDMA aided broadband multiuser } \\
\text { communications, WLANs and broadcasting was reviewed. }\end{array}$ \\
\hline 2006 & Myung et al. [128] & Introduced the concept of SC-FDMA for the wireless uplink. \\
\hline 2007 & Wilzeck et al. [132] & $\begin{array}{l}\text { Conceived a MIMO-SC-FDMA system model and analyzed the effect of } \\
\text { carrier frequency offsets. }\end{array}$ \\
\hline 2008 & Berardinelli et al. [122] & $\begin{array}{l}\text { A comparative performance study of OFDMA and SC-FDMA was carried } \\
\text { out. }\end{array}$ \\
\hline \multirow{2}{*}{2010} & Ciochina \& Sari [133] & Another comparative study of OFDMA versus SC-FDMA was offered. \\
\hline & Benvenuto et al. [123] & $\begin{array}{l}\text { Provided a comprehensive review of nonlinear FDE in the context of single- } \\
\text { carrier modulation. }\end{array}$ \\
\hline 2012 & Dalakas et al. [134] & OFDMA and SC-FDMA were compared for satellite uplink. \\
\hline
\end{tabular}

However, the full benefit of the SC-FDE was gradually realized after Sari et al. [125] proposed it as a low-complexity solution to digital terrestrial TV broadcasting, which was reviewed by Falconer et al. [126], [127]. The concept of SC-FDMA as a DFTprecoded OFDMA arrangement was proposed as a multipleaccess scheme for the LTE system by Myung et al. [128] as well as by the Pan-European Wireless INitiative NEw Radio (WINNER) project [129] in the context of the uplink of wide area cellular systems. A comprehensive review of nonlinear FDE in the context of single-carrier modulation was provided in [123].

\section{B. MC-CDMA Aided STSK}

MC-CDMA-aided STSK was conceived in [12] for mitigating the performance erosion of the classic STSK scheme in dispersive channels, while supporting multiple users. The codewords generated by the STSK scheme are appropriately spread across the frequency-domain (FD) and transmitted over a number of parallel frequency-flat subchannels.

1) Transmission Protocol: Let us consider an MC-CDMA aided STSK system having $M$ transmit and $N$ receive AEs and communicating over frequency-selective Rayleigh fading channels, whose transmitter architecture is shown in Fig. 11. Furthermore, $N_{c}$ subcarriers are employed by the MC-CDMA modem for transmitting $N_{p}$ STSK codewords. In general, $N_{c}$ is related to $N_{p}$ by: $N_{c}=\left(N_{p} \times S_{f}\right)$. However, it is possible to spread each of the codewords across all the subcarriers, where $S_{f}=N_{c}$ [94]. As seen in Fig. 11, STSK codewords $\boldsymbol{X}^{u}$ are generated from the users' source information, which are further spread across the FD and are then mapped to a number of sub-

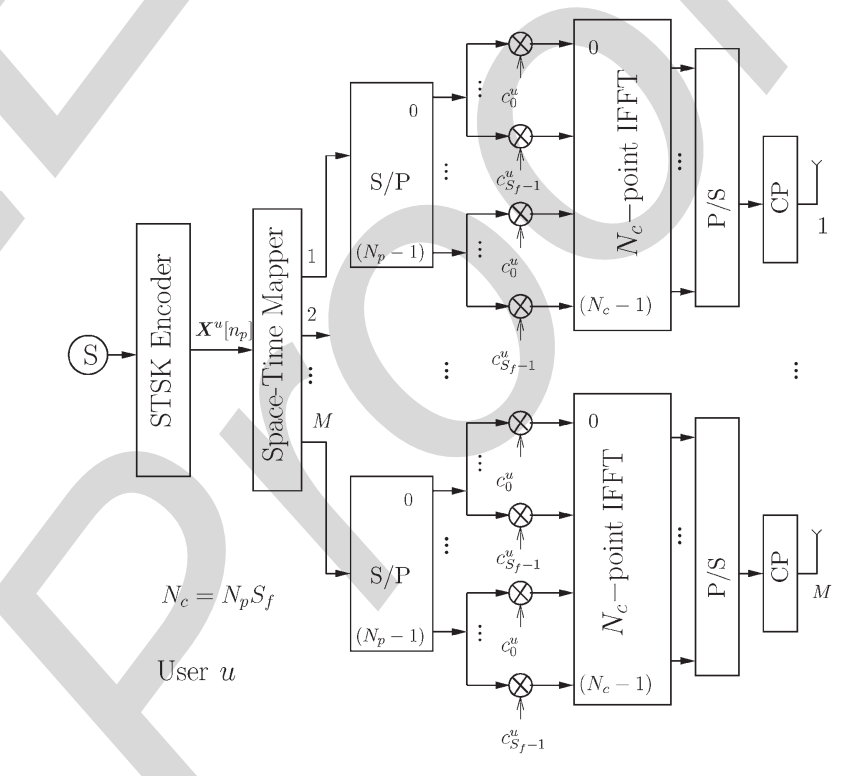

Fig. 11. Transmission model of the MC-CDMA aided multiuser STSK scheme of [12] corresponding to user $u$. Following space-time mapping, each of the TA symbols is spread across the FD before being mapped to the subcarriers.

carriers, before being transmitted using $M$ transmit AEs over $T$ time slots. A number of $N_{p}$ space-time codewords generated by the STSK encoder are spread across all the $N_{c}$ subcarriers by the user-specific spreading sequence, $\boldsymbol{C}^{u}=\left[c_{0}^{u}, c_{1}^{u}, \ldots, c_{S_{f}-1}^{u}\right]$, where $S_{f}$ represents the spreading factor and $u$ is the user index. To be more specific, assuming the relationship of $N_{c}=$ $\left(N_{p} \times S_{f}\right)$, FD spreading of the $N_{p}$ codeword symbols results in $N_{c}$ number of FD symbols of the spread codewords denoted 


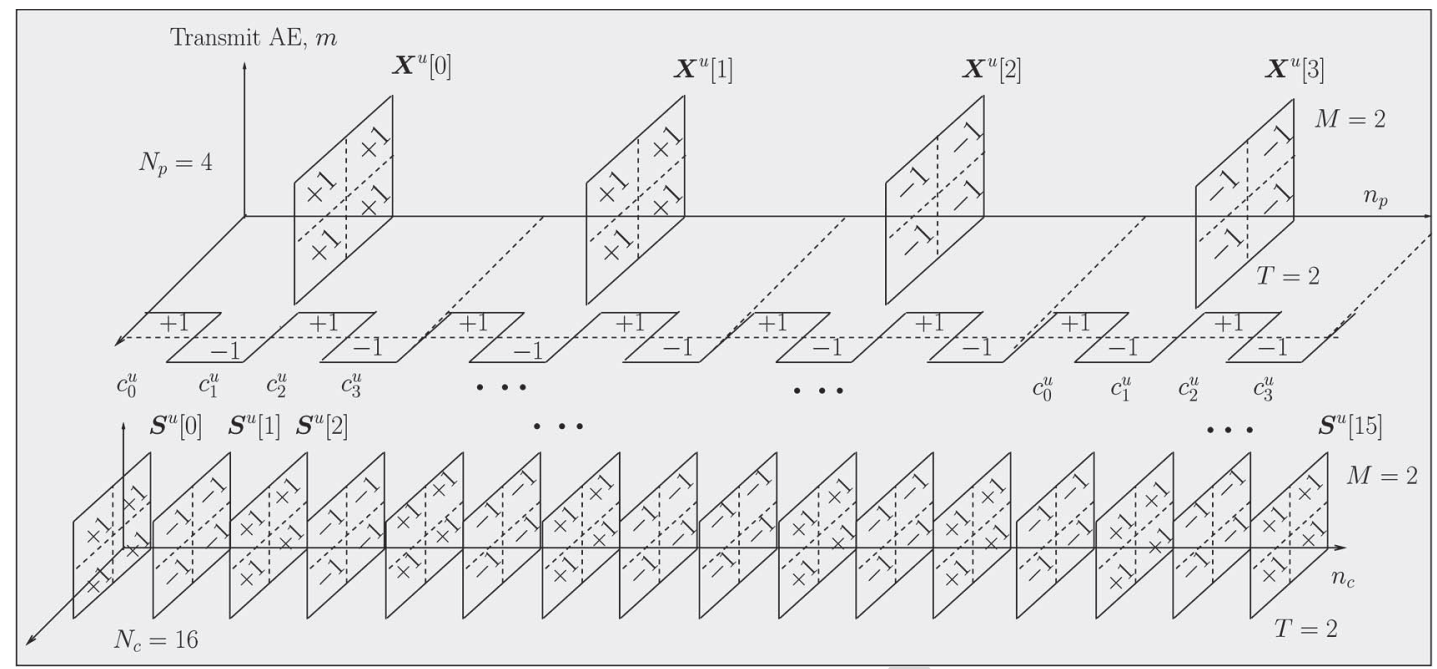

Fig. 12. FD spreading example of the STSK codewords in MC-CDMA-aided STSK system. 4 STSK codewords $\boldsymbol{X}^{u}[0], \boldsymbol{X}^{u}[1], \boldsymbol{X}^{u}[2]$, and $\boldsymbol{X}^{u}[3]$ are considered, which are spread in FD by the user-specific spreading sequence $\boldsymbol{C}^{u}=[+1,-1,+1,-1]$, resulting in 16 spread space-time matrices, which are denoted by $\boldsymbol{S}^{u}[0]$, $S^{u}[1], \ldots, S^{u}[15]$, respectively.

by $\boldsymbol{S}^{u}$. After the IDFT operation of Fig. 11, CP of appropriate length are incorporated for the sake of eliminating the effects of ISI. The mapping of the STSK codewords to the $N_{c}$ subcarriers is illustrated by the following example.

Example 1: FD spreading and STSK symbol mapping to MC-CDMA subcarriers: $N_{c}=16, S_{f}=4$

For the sake of exemplifying the FD spreading operation and the subsequent mapping of the spread codeword symbols to the orthogonal subcarriers, consider an MCCDMA aided STSK scheme employing $N_{c}=16$ subcarriers and a FD-spreading factor $S_{f}=4$. We will then take $N_{p}=N_{c} / S_{f}=16 / 4=4$ STSK codewords of a particular user $u$ and place them in parallel, as shown in Fig. 12. Let the 4 codewords be denoted by:

$$
\left[\boldsymbol{X}^{u}[0], \boldsymbol{X}^{u}[1], \boldsymbol{X}^{u}[2], \boldsymbol{X}^{u}[3]\right] .
$$

Let us further assume for example that the spreading sequence corresponding to user $u$ is:

$$
\boldsymbol{C}^{u}=[+1,-1,+1,-1] .
$$

Each of the codewords $\boldsymbol{X}^{u}$ will now be multiplied by each single chip of the spreading sequence $\boldsymbol{C}^{u}$, resulting in $4 \times 4=16$ spread codewords. More explicitly, $\boldsymbol{X}^{u}[0]$ multiplied by the chips will produce 4 spread codewords denoted by:

$$
\begin{aligned}
\boldsymbol{S}^{u}[0] & =c_{0}^{u} \boldsymbol{X}^{u}[0]=+1 \times \boldsymbol{X}^{u}[0], \\
\boldsymbol{S}^{u}[1] & =c_{1}^{u} \boldsymbol{X}^{u}[0]=-1 \times \boldsymbol{X}^{u}[0], \\
\boldsymbol{S}^{u}[2] & =c_{2}^{u} \boldsymbol{X}^{u}[0]=+1 \times \boldsymbol{X}^{u}[0], \\
\text { and } \quad \boldsymbol{S}^{u}[3] & =c_{3}^{u} \boldsymbol{X}^{u}[0]=-1 \times \boldsymbol{X}^{u}[0] .
\end{aligned}
$$

Thus the 4 codewords $\left[\boldsymbol{X}^{u}[0], \boldsymbol{X}^{u}[1], \boldsymbol{X}^{u}[2], \boldsymbol{X}^{u}[3]\right]$ will produce a total of 16 spread codewords denoted by:

$$
\left[\boldsymbol{S}^{u}[0], \boldsymbol{S}^{u}[1], \ldots, \boldsymbol{S}^{u}[15]\right] .
$$

In Fig. 12, we assume all the STSK codewords to be $(2 \times$ 2) - element matrices, i.e., we assume $M=2, T=2$. We also consider, for the sake of simplicity, all the elements of the STSK codewords $\boldsymbol{X}^{u}[0]$ and $\boldsymbol{X}^{u}[1]$ to be +1 , while those of $\boldsymbol{X}^{u}[2]$, and $\boldsymbol{X}^{u}[3]$ to be -1 . As a result, all the elements of the spread codewords $S^{u}[0], S^{u}[1], S^{u}[2]$, and $\boldsymbol{S}^{u}[3]$ are $+1,-1,+1$ and -1 , respectively. By contrast, all the elements of for example $S^{u}[8], S^{u}[9], S^{u}[10]$, and $S^{u}[11]$ are $-1,+1,-1$ and +1 , respectively.

The resultant 16 spread codeword symbols will then be mapped to the $N_{c}=16$ subcarriers using the IDFT operation.

2) Receiver: The signal received after transmission over a dispersive channel is demodulated by Fourier-transforming it after removal of CP. After CP removal and $N_{c}$-point DFT, the FD signal received is given by:

$$
\boldsymbol{Y}\left[n_{c}\right]=\underbrace{\tilde{\boldsymbol{H}}^{v}\left[n_{c}\right] \boldsymbol{S}^{v}\left[n_{c}\right]}_{\text {desired signal }}+\underbrace{\sum_{\substack{u=1 \\ u \neq v}}^{U} \tilde{\boldsymbol{H}}^{u}\left[n_{c}\right] \boldsymbol{S}^{u}\left[n_{c}\right]}_{\text {MUI }}+\underbrace{\boldsymbol{V}\left[n_{c}\right]}_{\text {noise }},
$$

where each element of the FD channel transfer matrix $\tilde{\boldsymbol{H}}^{u}\left[n_{c}\right] \in \mathbb{C}^{N \times M}$ is a diagonal matrix given by $\tilde{\boldsymbol{H}}_{n, m}^{u}=$ $\operatorname{diag}\left\{\tilde{h}_{n, m}^{u}[1], \tilde{h}_{n, m}^{u}[2], \ldots, \tilde{h}_{n, m}^{u}\left[N_{c}\right]\right\} \in \mathbb{C}^{N_{c} \times N_{c}}$, whose diagonal elements represent the FD channel transfer function between the $m$-th transmit and the $n$-th receive AE. Both singleuser detectors (SUDs) and multiuser detectors (MUDs) may be employed for the detection of the different users' signals [117]. Since the orthogonality amongst the different users' signals is destroyed by dispersive channels, the MUI cannot be eliminated if a low-complexity SUD is employed. The MUI degrades the resultant BER performance both in the downlink (DL) and the uplink (UL) multiuser scenarios. By contrast, the MUD jointly detects the information of all the users and hence an improved BER performance may be attained, albeit at the cost of imposing an increased decoding complexity. The source information of users in a particular space-time block indexed by $n_{p}$ is spread 


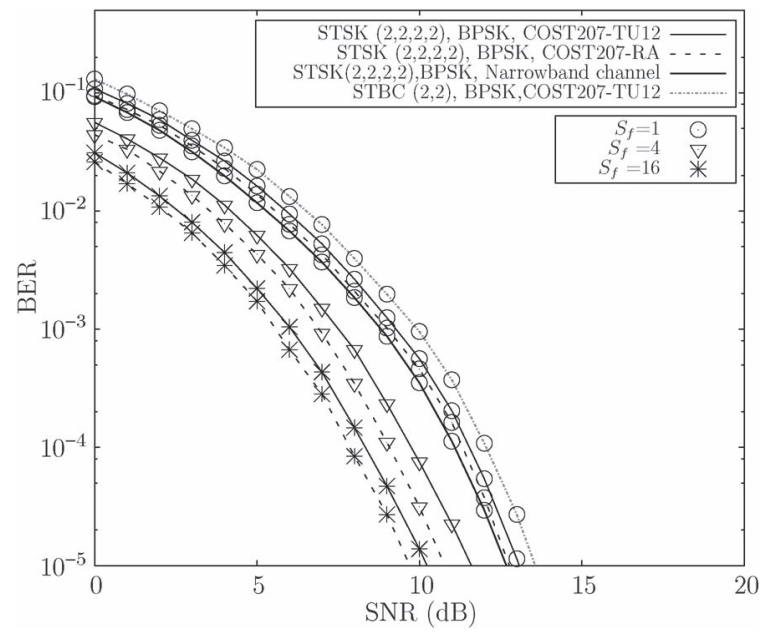

Fig. 13. Performance of single-user uncoded MC-CDMA STSK system of Fig. 11 both in narrowband and in dispersive channels. The performance of the MC-CDMA aided $\mathcal{G}_{2}-\operatorname{STBC}(M, N)=(2,2)$, BPSK and $\mathcal{G}_{2}-\operatorname{STBC}(2,2)$, QPSK is also shown as benchmarker.

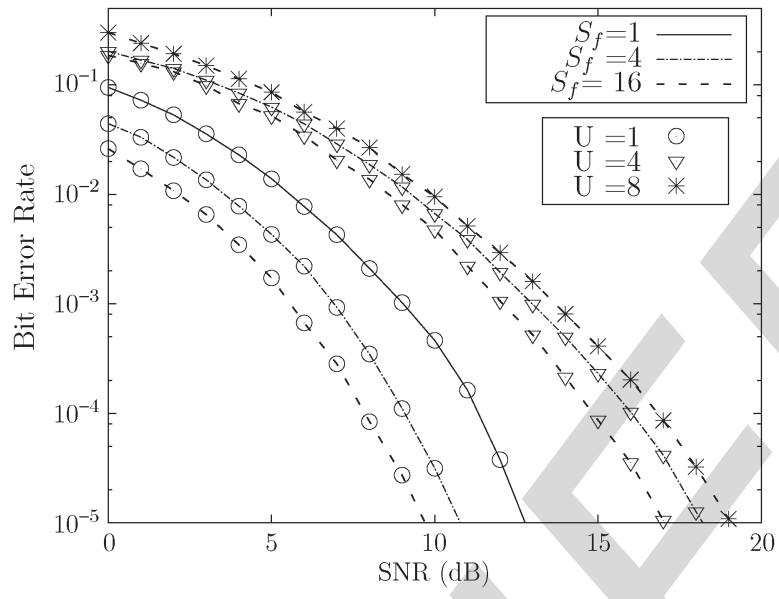

Fig. 14. BER performance of MC-CDMA aided STSK $(2,2,2,2)$, BPSK for the DL COST207-RA channel using different $S_{f}$ supporting $U$ users [12].

over $S_{f}$ number of outputs from $\overline{\boldsymbol{Y}}\left[n_{p} S_{f}\right]$ to $\overline{\boldsymbol{Y}}\left[\left(n_{p}+1\right) S_{f}-1\right]$. The single-user detector combines the signal energy spread over the different subcarriers employing a combining method, such as orthogonality-restoring combining (ORC) [117], EGC [22], [117] or MRC [22], [117]. Using MRC, the decision variables of the desired user $v$ may be obtained as [117]:

$$
\boldsymbol{z}^{v}\left[n_{p}\right]=\sum_{s_{f}=0}^{\left(S_{f}-1\right)} \boldsymbol{g}^{v}\left[n_{p} S_{f}+s_{f}\right] \boldsymbol{Y}\left[n_{p} S_{f}+s_{f}\right],
$$

where $\boldsymbol{g}^{v}\left[n_{p} S_{f}+s_{f}\right]$ is given by $\boldsymbol{g}^{v}\left[n_{p} S_{f}+s_{f}\right]=c_{s_{f}}^{v}\left[\tilde{\boldsymbol{H}}^{v}\left[n_{p} S_{f}+s_{f}\right]\right]^{H}$. Hence, the source bits of user $v$ may be estimated as [8], [13]

$$
\left(q^{v}\left[n_{p}\right], l_{c}^{v}\left[n_{p}\right]\right)=\underset{q^{v}, l_{c}^{v}}{\arg \min }\left\{\left\|\overline{\boldsymbol{z}}^{v}\left[n_{p}\right]-\boldsymbol{\chi} \boldsymbol{K}_{q^{v}, l_{c}^{v}}^{v}\right\|^{2}\right\} .
$$

A new single-stream maximum-likelihood (ML) MUD (ML-MUD) may also be employed [12] for the joint detection of the user signals, which amalgamates both the single-stream ML detector of [8], [10] and the MUD of [117].

The performances achievable by the MC-CDMA-aided STSK under both a single-user and a multiuser scenario are illustrated

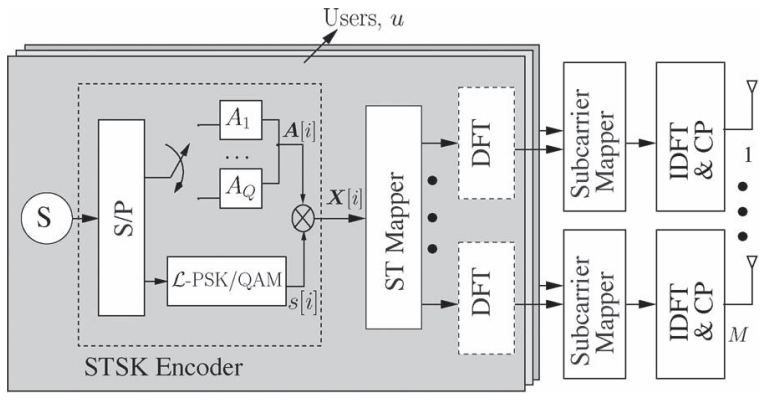

Fig. 15. Transmission model of the SC-FDMA aided STSK scheme. In OFDMA the dotted "DFT $N_{d}$ " block of the transmitter does not exist. The STSK mapper selects one out of the Q dispersion matrices along with one constellation symbol and the resultant space-time codewords are passed in different time slots through the OFDMA or SC-FDMA based multi-user transmission system, before being transmitted by the transmit AEs.

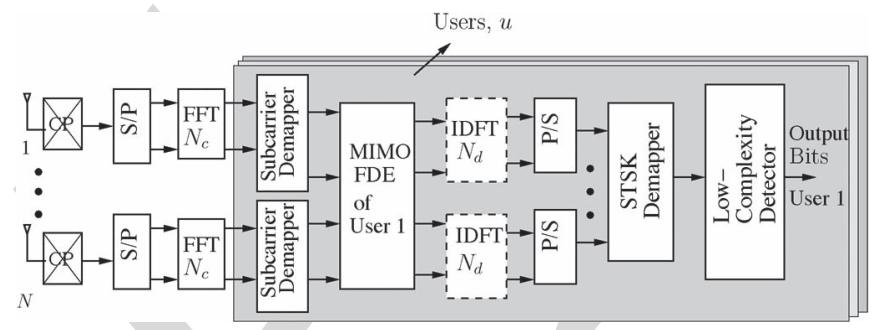

Fig. 16. Receiver model of the SC-FDMA aided STSK scheme. In OFDMA the dotted "IDFT $N_{d}$ " block of the receiver does not exist. Since a single dispersion matrix is selected in each transmission block, a low-complexity single-stream ML detector can be employed.

in Figs. 13 and 14, respectively. As expected, the MC-CDMA STSK scheme is capable of maintaining its superior multiple antenna gain in both the rural area (RA) scenario modeled by the COST207-RA channel as well as in the TU scenario characterized by the COST207-TU12 model. Furthermore, the single-user MC-CDMA aided STSK scheme shows improved performance upon increasing $S_{f}$ both for the COST207-RA and COST207TU12 channels owing to the fact that a FD spreading sequence may still remain recoverable, even when some of its chips are corrupted, while the BER is degraded under multiuser scenarios as a result of the increased MUI imposed by multiple users.

\section{OFDMA/SC-FDMA-Aided Multiuser STSK}

An OFDMA/SC-FDMA-aided STSK scheme was also conceived in [13] which is capable of attaining a superb diversitymultiplexing trade-off even in a multipath environment, whilst additionally supporting multiuser transmissions and maintaining a low peak-to-average power ratio (PAPR) in uplink SCFDMA-aided STSK scenarios. Since OFDMA and SC-FDMA have been adopted for the DL and the UL of the Long Term Evolution (LTE)-Advanced standard, respectively [135], [136], OFDMA/SC-FDMA assisted STSK systems are advocated in [13].

1) Transmitter and Receiver Model of the OFDMA/SCFDMA-Aided STSK: The transmitter and the receiver architectures of an OFDMA/SC-FDMA STSK system are depicted in Figs. 15 and 16, respectively. As shown in Fig. 15, we employ frame based transmission after generating the space-time blocks $\boldsymbol{x}^{u}\left[n_{d}\right]$ for a particular user $u$. In particular, $N_{c}$ 


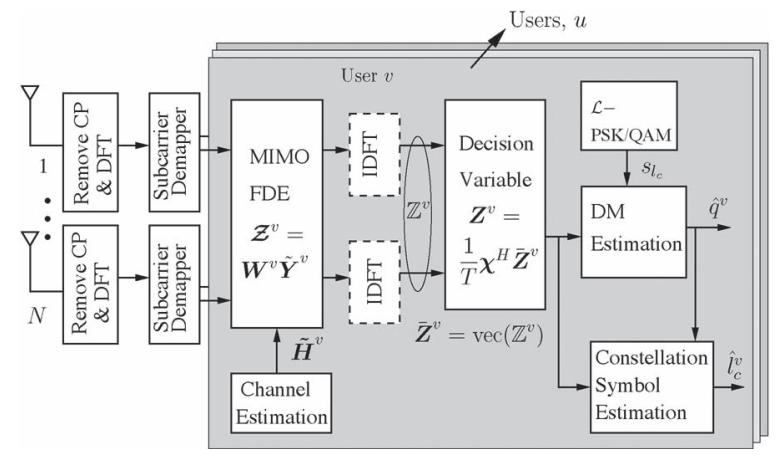

Fig. 17. The receiver architecture of the OFDMA/SC-FDMA-aided STSK scheme employing the reduced-complexity detector of [137]. The dotted "IDFT" block does not exist in the OFDMA-aided STSK receiver.

sub-carriers are used for transmitting a frame, each frame consisting of $N_{d}$ STSK blocks. To be more specific, the data stream $\tilde{\boldsymbol{x}}_{m, T_{i}}^{u}$ to be transmitted from the transmit AE $m$ at a specific time interval $T_{i}$ is first DFT-precoded by the $N_{d}$-point DFT block; in case of OFDMA, however, this step is not required. Then the FD symbols output from the $N_{d}$-point DFT block of the SC-FDMA STSK scheme (or the direct FD STSK codeword symbols of the OFDMA STSK scheme) are mapped to $N_{c}$ subcarriers with $N_{c}=\left(N_{d} \times U\right)$, where the sub-carrier allocation may be in contiguous (localized FDMA or LFDMA) [128] or in an interleaved (IFDMA) [128] fashion. Assuming perfect synchronization at the receiver of Fig. 16 and after removing the $\mathrm{CP}$, the received signal is first Fourier transformed and the resultant FD subcarrier signals are then demapped and equalized in FD by the "MIMO FDE block" of Fig. 16. Following MIMO FDE, the received signal is passed through the " $N_{d}$-point IDFT" block of the desired user $v$ and detected in TD using the single-stream ML detector of [13]. In case of OFDMA, however, " $N N_{d}$-point IDFT" does not exist and the signal is detected in FD by the single-stream ML detector of [13] and as such, the OFDMA scheme cannot benefit from the potential diversity provided by the DFT based precoding stage. It is worth mentioning at this point that under the idealized assumption of perfect synchronization, perfect orthogonality of the users using different sub-carriers, the OFDMA/SC-FDMAaided STSK scheme is decontaminated from MUI. However, the symbols transmitted by a given user in the context of both the LFDMA and IFDMA scheme with MMSE equalization experience some form of self-interference (SI) [110]. The singlestream based ML detector for estimating the source bits of user $v$ from the decision variable $z^{v}$ of the OFDMA/SC-FDMA aided STSK scheme may be formulated as [13]:

$$
\left(\hat{q}^{v}, \hat{l}_{c}^{v}\right)=\underset{q, l_{c}}{\arg \min }\left\|\bar{z}^{v}\left[n_{d}\right]-\chi \boldsymbol{k}_{q, l_{c}}^{v}\right\|^{2},
$$

where $\boldsymbol{k}_{q, l_{c}}^{v}$ is the equivalent transmit signal vector corresponding to user $v$ at indices $q$ and $l_{c}$. Note that the single-stream ML detector of (21) does not explicitly contain either the FD channel transfer function or the TD channel impulse response. Hence, data estimation using (21) involves a reduced number of multiplications and additions.

2) Reduced-Complexity Detector for the OFDMA/SCFDMA-Aided STSK: In order to further reduce the compu- tational complexity of the OFDMA/SC-FDMA-aided STSK scheme of [13], a reduced-complexity detector was conceived in [137]. The reduced-complexity detector of [137] was designed in the spirit of [48], while exploiting the reducedcomplexity behavior of the single-stream ML detector of [8], [13]. The schematic diagram of the OFDMA/SC-FDMA-aided STSK receiver employing the reduced-complexity detector is shown in Fig. 17. This detector first employs MIMO MMSE FDE, followed by the "IDFT" processing, although the "IDFT" block does not exist in case of the OFDMA-aided STSK. Then the indices of the activated DM, $q^{v}$ and of the constellation symbol, $l_{c}^{v}$ corresponding to a particular user $v$ are estimated.

Following the above-mentioned elaborations on the detector's operation obeying the architecture portrayed in Fig. 17, each of the steps followed by the detector may be summarized as in Algorithm 1.

Algorithm 1: A reduced-complexity detector conceived for OFDMA/SC-FDMA-aided STSK

1) Given the STSK parameters $(M, N, T, Q, \mathcal{L})$ and the DMs in terms of the dispersion character matrix (DCM) $\boldsymbol{\chi}$, the signals received by the AEs are input to MIMO FDE after OFDM demodulation and subcarrier demapping;

2) After multiplication by the MMSE weight matrix $\boldsymbol{W}^{v}$, the signal is passed through the "IDFT" block of Fig. 17. For OFDMA, there is no "IDFT" block, as seen in Fig. 17;

3) The vectorial stacking based linearization of [5] is employed: $\overline{\boldsymbol{Z}}^{v}=\operatorname{vec}\left(\mathbb{Z}^{v}\right)$;

4) The decision variable is then obtained as: $\boldsymbol{Z}^{v}=\frac{1}{T} \boldsymbol{\chi}^{H} \overline{\boldsymbol{Z}}^{v}$;

5) The index $\hat{q}^{v}$ of the DM activated and the index $\hat{l}^{v}$ of the constellation symbol are computed separately;

6) The index $\hat{q}^{v}$ of the DM activated is evaluated as:

$$
\begin{aligned}
\hat{q}^{v}=\underset{q \forall l_{c}^{\prime}}{\arg \max }\left[2 \left\{\left|\mathfrak{R}\left(z_{q}^{v}\right)\right|\left|\mathfrak{R}\left(s_{l_{c}^{\prime}}\right)\right|\right.\right. \\
\left.\left.+\left|\mathfrak{I}\left(z_{q}^{v}\right) \| \mathfrak{I}\left(s_{l_{c}^{\prime}}\right)\right|\right\}-\left|s_{l_{c}^{\prime}}\right|^{2}\right]
\end{aligned}
$$

7) The index $\hat{l}^{v}$ of the constellation symbol is found by evaluating:

$$
\hat{l}^{v}=\underset{l}{\arg \min }\left|z_{\hat{q}}^{v}-s_{l}\right|
$$

8) Thereby the source bits of user $v$ have now been estimated.

The reduced-complexity detector is a promising candidate for employment in OFDMA/SC-FDMA-aided STSK transmissions over frequency-selective channels even under multiuser scenarios in terms of its performance versus complexity trade-off.

\section{SucCessive RELAYING-Aided DisTRIBUTED MC STSK}

In Sections III-B, IV-B, and IV-C, we have investigated the schemes incorporating OFDM, MC-CDMA, and OFDMA/ SC-FDMA based transmissions of the multiple-antenna signals 


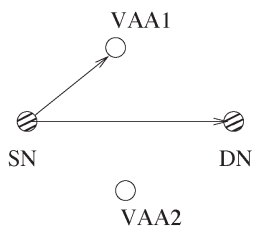

(a)

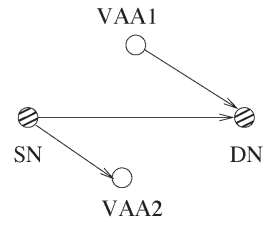

(b)

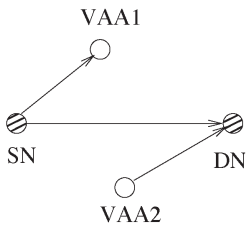

(c)
Fig. 18. Transmission protocol of SR aided cooperation during different time slots [14], [19].

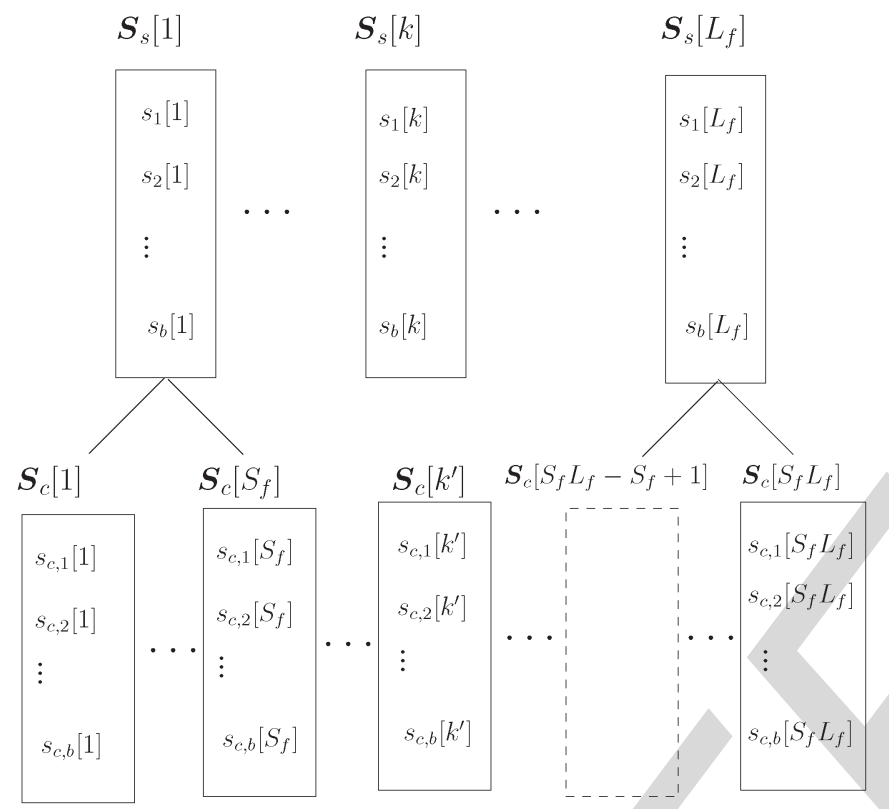

Fig. 19. Illustration of the transmission protocol of the SN of Fig. 18. The $L_{f}$ transmit blocks $S_{S}[k]$ of a transmission frame are spread in FD by the MCCDMA modem using the user-specific spreading sequence $\boldsymbol{C}_{A}^{u}$ or $\boldsymbol{C}_{B}^{u}$ depending on whether frame-A or frame-B is being transmitted, which results in $S_{f} L_{f}$ spread blocks $\boldsymbol{S}_{c}\left[k^{\prime}\right]$.

relying on the STSK system philosophy. However, in those sections, we have focussed our attention on MIMO scenarios, where multiple co-located antennas are employed both at the transmitter as well as at the receiver. Hence for simplicity we refer to these as the family of co-located MIMO schemes, which are potentially susceptible to the detrimental effects of inter-antenna correlation due to the insufficient separation among the antennas. For the sake of mitigating the deleterious effects imposed by the inter-antenna correlation, in this section, we consider SR aided coherent-versus-noncoherent MC STSK schemes.

\section{A. Coherent SR Aided Cooperative MC STSK}

The typical transmission protocol of the SR scheme [19] is shown in Fig. 18, where the distributed RNs form a pair of virtual antenna arrays (VAAs) and the source node (SN) successively transmits its FD spread signals to one of the VAAs in addition to directly transmitting it to the destination node (DN).

1) Transmission Protocol of the $S N$ : The transmission regime of the $\mathrm{SN}$ is illustrated in Fig. 19. The SN first attaches the cyclic redundancy checking (CRC) bits to its information bits and transmits them both to the $M$ RNs

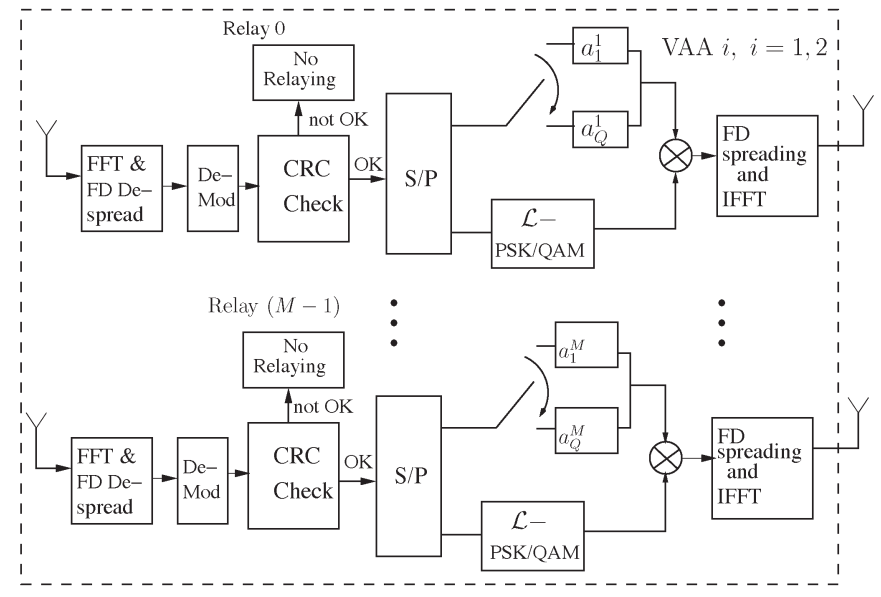

Fig. 20. Architecture and relaying principle of each of the VAAs.

of a VAA as well as to the DN in each of its broadcast phases, as seen from Fig. 19. The CRC-protected bits are then mapped to $\mathcal{L}^{\prime}$-PSK/QAM symbols, which then form the symbol blocks $\boldsymbol{S}_{S}[k] \triangleq\left[s_{1}[k], \ldots, s_{b}[k]\right]^{T} \in \mathbb{C}^{b \times 1}$ of $b$ symbols, where $k(1,2, \ldots)$ denotes the block index. A frame is then defined by $L_{f}$ number of the above-mentioned symbol blocks, where $L_{f}$ represents the frame length. The frames are then divided into two groups-those that are broadcast when VAA1 of Fig. 18 is receiving are termed as frame- $\mathrm{A}$, while those being broadcast when VAA2 is receiving are referred to as frame-B. Frame-A is then spread in FD by the $S_{f}$-length user-specific spreading sequence $\boldsymbol{C}_{A}^{u}$, while frame-B by $\boldsymbol{C}_{B}^{u}$. This spreading operation results in the spread blocks $\boldsymbol{S}_{C}\left[k^{\prime}\right]=C_{A}^{u}\left[s_{f}\right] \boldsymbol{S}_{S}[k] \in \mathbb{C}^{b \times 1}$ and $\boldsymbol{S}_{c}\left[k^{\prime}\right]=C_{B}^{u}\left[s_{f}\right] \boldsymbol{S}_{s}[k] \in \mathbb{C}^{b \times 1}$, respectively, depending on which frame is being transmitted. Assuming the spread frame length $\left(L_{f} \times S_{f}\right)$ to be a multiple of the number $N_{c}$ of subcarriers, each frame is mapped to the subcarriers using the $N_{c}$-point IDFT and then it is transmitted after appending the CPs.

2) The VAA: The constituent RNs of each VAA activate CRC based selective decode-and-forward (DF) relaying. The relaying principle of each of the VAAs is portrayed in Fig. 20. Each of the two VAAs is composed of $M$ RNs and the signal received at each RN of a VAA is decoded employing FD MCCDMA despreading. If the signal at the $\mathrm{RN}$ is deemed to be correctly decoded by the CRC, then it is allowed to relay. The RN then re-encodes the decoded bits to STSK codewords in accordance with the relationship of $b \cdot \log _{2} \mathcal{L}^{\prime}=\log _{2}(\mathcal{L} \cdot Q)$, where $\mathcal{L}$ is the modulation order of the constellation employed by the STSK [14]. In Fig. 20, the $m$-th row of the DM $\boldsymbol{A}_{q}(q=$ $1, \ldots, Q)$ is represented by $a_{q}^{m}$. After appropriate re-encoding, the $m$-th RN transmits the $m$-th row of the resultant STSK codeword after MC-CDMA based FD-spreading and IDFT operation, provided that no decoding error is identified by the CRC. Otherwise, the RN refrains from relaying.

3) Joint Detection at the $D N$ : The DN then jointly detects the signals received via the $\mathrm{SN}$-to-DN and the VAA-to-DN links using the single-stream based joint maximum-likelihood (ML) detector of [14]. To elaborate a little further, the DN employs the double-frame matched filter of [138] for detection of the two replicas of the same signal. Specifically, a filter matched to 
$C_{A}^{u}$ is employed during frame-A, whereas a filter matched to $C_{B}^{u}$ is employed during the next consecutive frame-B transmission.

The SR regime discussed above assists in recovering the half-duplex throughput loss at the cost of imposing inter-VAA interference. Furthermore, the signal also interferes with other stream arriving at the DN [19], [20]. The problem of InterVAA interference is eliminated by invoking the proposed CRC based selective DF cooperation along with the specific FD despreading regime used, while the interference at the DN is mitigated by using the double-frame-based chip-waveform matched filter of [138].

A close observation of the SR strategy reveals that the two replicas of the same frame arrive at the DN in the consecutive phases through the direct SN-DN link and via the VAA links. Hence, the joint detection is carried out over two consecutive frames of the received signal. The joint ML detector conceived for estimating the bits of frame-A may be formulated as [14], [18]:

$$
\left(\hat{q}[k], \hat{l_{c}}[k]\right)=\underset{q, l_{c}}{\arg \min }\left\{\left\|\boldsymbol{Z}^{A}[k]-\boldsymbol{H}_{J}^{A}[k] \overline{\boldsymbol{S}}_{s}^{q, l_{c}}\right\|^{2}\right\},
$$

where $\|\bullet\|$ represents the Euclidean norm of the matrix “ $\bullet$," $\boldsymbol{S}_{s}^{q, l_{c}}$ and $\overline{\boldsymbol{S}}_{s}^{q, l_{c}}$ are the legitimate values of the symbol blocks $\boldsymbol{S}_{s}[k]$ and $\overline{\boldsymbol{S}}_{S}[k]$ specified by the indices $\left(q, l_{c}\right)$ during symbol duration $k$, while the combined FD channel transfer matrix is defined as [14], [18]:

$$
\boldsymbol{H}_{J}^{A}[k] \triangleq\left[\begin{array}{c:c}
\bar{h}_{s d}^{A}[k] \boldsymbol{I}_{b} & \mathbf{0}_{b \times Q} \\
\hdashline \mathbf{0}_{T \times b} & \left.\overline{\boldsymbol{H}}_{v_{1} d}^{A}\left[k+L_{f}\right] \boldsymbol{\chi}\right] \ldots . .
\end{array}\right] \in \mathbb{C}^{(b+T) \times(b+Q)},
$$

with two submatrices expressed by $\bar{h}_{s d}[k] \boldsymbol{I}_{b} \in \mathbb{C}^{b \times b}$ and $\overline{\boldsymbol{H}}_{v_{1} d}\left[k+L_{f}\right] \boldsymbol{\chi} \in \mathbb{C}^{T \times Q}$, respectively, and two zero matrices.

We note that as a benefit of employing SR, the information is received by the DN both from the direct SN-DN link as well as from the VAA-DN link during both the broadcast and the cooperation phase. This leads to the total normalized throughput of [139]

$$
\begin{aligned}
R & =\frac{b \cdot \log _{2} \mathcal{L}^{\prime}+T \log _{2}(\mathcal{L} \cdot Q)}{b+T} \\
& =\frac{2 \log _{2}(\mathcal{L} \cdot Q)}{b+T}[\text { bits/symbol duration }]
\end{aligned}
$$

which is twice that of the corresponding half-duplex scheme [14], [18], [19]. This benefit, however, is achieved at the cost of supporting less users, because the available number of spreading sequences becomes less as a consequence of employing SR.

\section{B. Noncoherent SR Aided Cooperative MC STSK}

In order to overcome the performance degradation imposed by channel estimation (CE) errors, a cooperative MC DSTSK scheme was studied in [14], which retained all the fundamental benefits of the coherent scheme. It was shown in [14] that the CRC-activated coherent scheme potentially benefits from a higher diversity gain than either the conventional DF or the noncooperative schemes, whilst attaining an increased throughput, which is an explicit advantage of using SR. However, the differ- ential scheme suffers from a performance penalty of about $3 \mathrm{~dB}$ compared to the perfect-channel-state information (CSI) aided coherent scheme owing to the inherent noise doubling process of differential encoding. On the other hand, the cooperative coherent scheme's performance was severely degraded by the CE errors. Moreover, the coherent scheme requires the transmission of pilot symbols in addition to the CRC overhead. Hence, the differential multi-carrier cooperative STSK system may be considered an attractive candidate for cooperative MIMO-aided multi-carrier communications.

The transmission regime of the noncoherent MC DSTSK scheme is portrayed in Fig. 21, where the identical architectures of the pair of VAA arrangements are explicitly shown. In particular, the following points are worth mentioning in the context of the distributed MC DSTSK.

1) The DMs used for the cooperative MC DSTSK scheme are directly generated unitary matrices as in [140], so that the employment of nonlinear Cayley transform of [8] may be avoided.

2) The differential encoding requires satisfying the simplifying condition [27] of $M=T$, so that the STSK codewords are $(M \times M)$-element square matrices.

3) Differential encoding of the multicarrier based system can be performed either in the TD (differential encoding across the consecutive symbols of the same sub-carrier) or in the FD (differential encoding across the symbols of the adjacent sub-carriers of the same MC-CDMA block). The TD approach is suitable for continuous transmissions, as opposed to the FD approach, which is useful for burst-based transmissions [141], [142].

\section{ML-MSDSD AND SOFT MAP-MSDSD-AIDED MC DSTSK}

In this section, both hard-decision as well as soft-decision based MSDSD is invoked for MC DSTSK-aided transmission over frequency-selective channels. Specifically, the DSTSK signaling blocks are generated by the original source bits of the hard-decision scheme or by the channel-encoded bits of the soft-decision system. The resultant space-time blocks are then appropriately mapped to a number of OFDM subcarriers. After OFDM-demodulation, the DSTSK signal may be noncoherently detected by a soft-decision-aided MAP-MSDSD detector [69].

Again, as investigated in [8], [14], DSTSK employing conventional differential detection (CDD) suffers from a typical $3 \mathrm{~dB}$ performance penalty in low-Doppler scenarios. Additionally, a high-mobility scenario characterized by a high Doppler frequency may result in an irreducible error floor [143]. The performance erosion of CDD may be mitigated by employing multiple-symbol differential detection (MSDD) [144]. MSDD is capable of efficiently exploiting the fading-plus-noise statistics of the channel for the joint detection of $\left(N_{w}-1\right)$ information symbols from $N_{w}$ number of consecutively received symbols, where $N_{w}$ is usually termed as the observation window size. However, the complexity associated with MSDD is typically high. Hence sphere decoding may be combined with MSDD for reducing the complexity and the resultant scheme is 


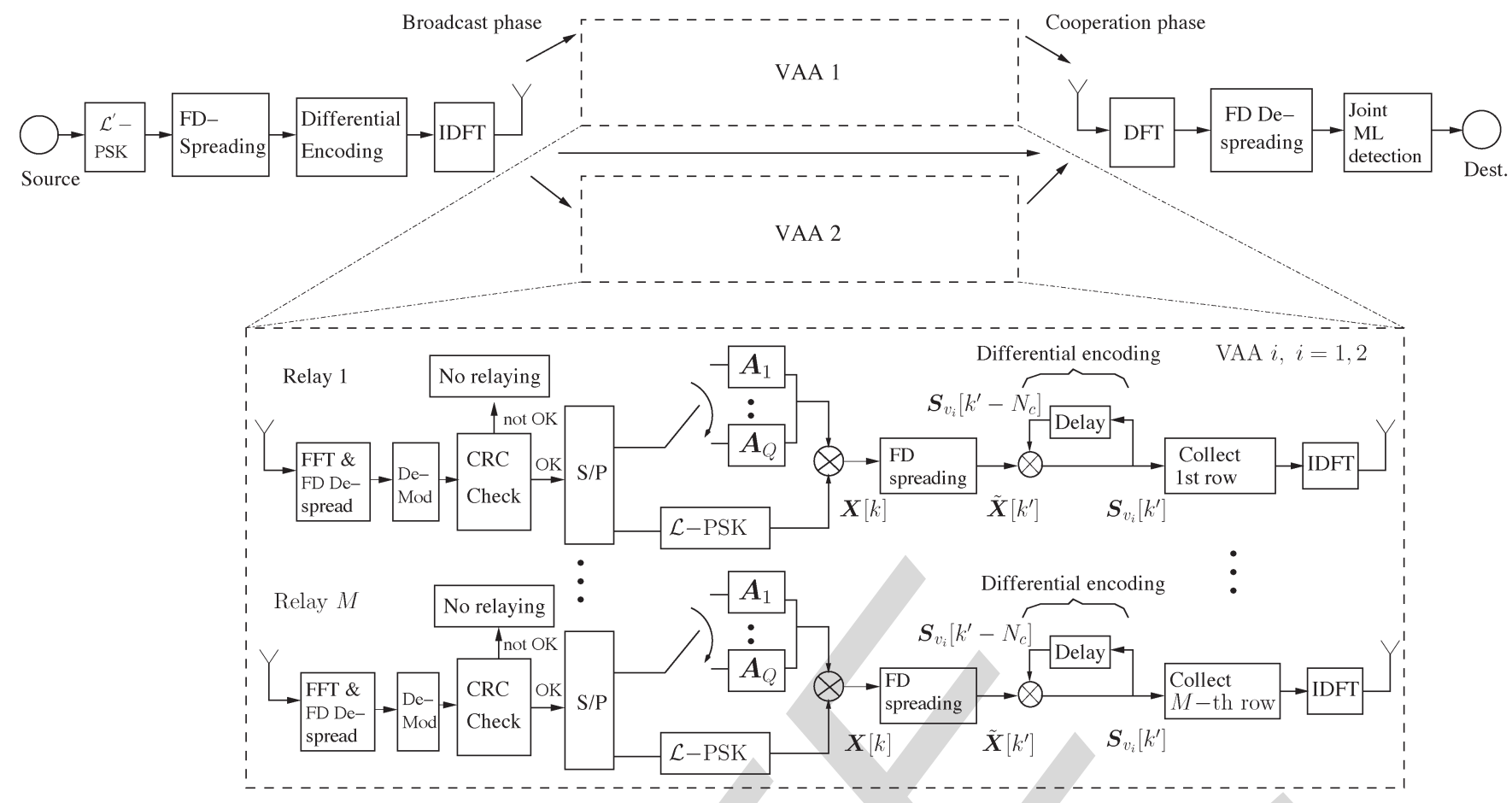

Fig. 21. Schematic of the distributed MC DSTSK employing CRC-based selective DF relaying [139].

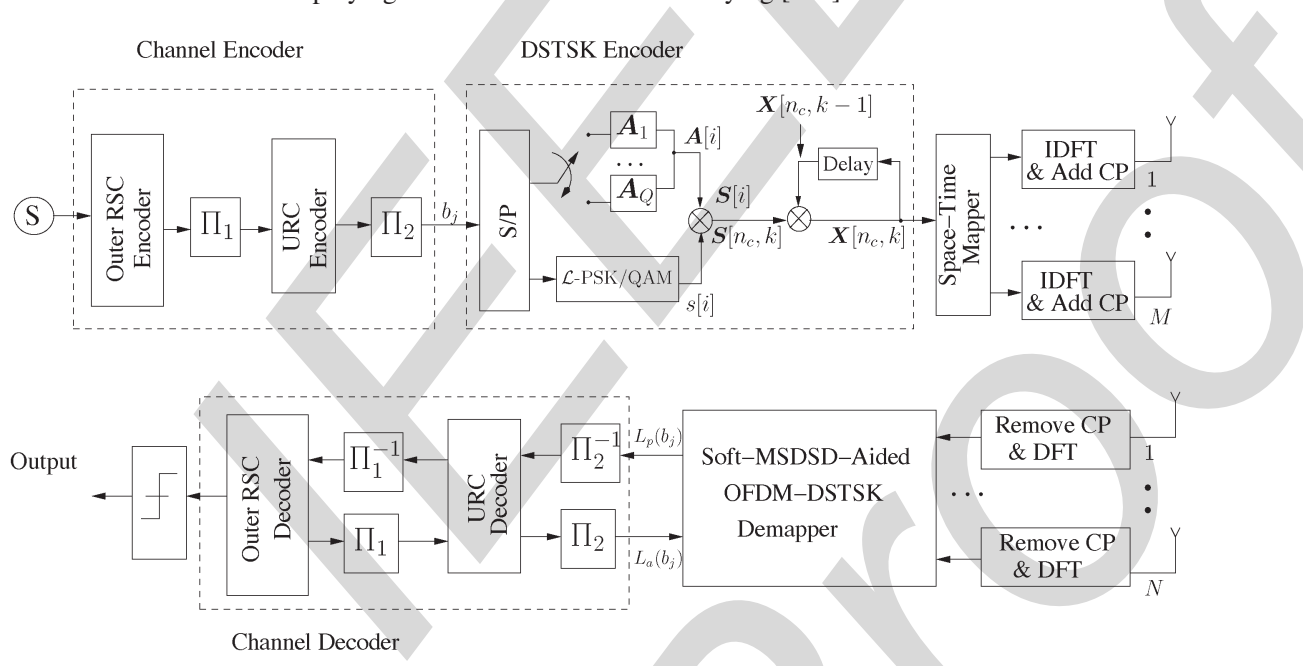

Fig. 22. Transceiver architecture of the concatenated channel-coding aided DSTSK scheme relying on the soft-MSDSD of [69] as the inner decoder. For harddecision scheme, the channel en/decoder block is absent and ML-MSDSD is invoked in place of MAP-MSDSD.

referred to as the MSDSD [145], [146]. On the other hand, the concept of differential space-frequency modulation (DSFM) employing MSDSD was conceived in [141] for exploiting both the achievable spatial- and frequency-domain diversity. Hence, the ML-MSDSD and soft-decision MSDSD designed for OFDM based MC DSTSK operating in frequency-selective channels is investigated in this section [69].

As shown in Fig. 22, the STSK codewords $S[i]$ of the MC DSTSK scheme are mapped to $N_{c}$ parallel subcarriers, before being differentially encoded. The codewords $\boldsymbol{S}\left[n_{c}, k\right]$ are thus differentially encoded to form the transmit blocks $\boldsymbol{X}\left[n_{c}, k\right](k=$ $0,1,2, \ldots)$ according to [141]

$$
\boldsymbol{X}\left[n_{c}, k\right]= \begin{cases}\boldsymbol{X}\left[n_{c}, k-1\right] \boldsymbol{S}\left[n_{c}, k\right], & k=1,2, \ldots \\ \boldsymbol{I}_{T} & k=0 .\end{cases}
$$

The DSTSK codewords are then transmitted after the $N_{c}$ - point inverse discrete Fourier transform (IDFT) operation and appropriate $\mathrm{CP}$ incorporation.

\section{A. ML-MSDSD for MC DSTSK}

The ML-MSDD scheme of [69] processes $N_{w}$ consecutively received space-time blocks of the $n_{c}$-th subcarrier given by $\boldsymbol{Y}^{w}\left[n_{c}, k\right] \triangleq\left[\boldsymbol{Y}^{T}\left[n_{c}, k-N_{w}+1\right], \ldots, \boldsymbol{Y}^{T}\left[n_{c}, k\right]\right]^{T}$ and finds the ML estimates $\boldsymbol{X}^{w}\left[n_{c}, k\right]$ of the corresponding $N_{w}$ transmitted blocks [146], [147], as defined by $\boldsymbol{X}^{w}\left[n_{c}, k\right]=$ $\left[\boldsymbol{X}^{T}\left[n_{c}, k-N_{w}+1\right], \ldots, \boldsymbol{X}^{T}\left[n_{c}, k\right]\right]^{T}$. Since the differentially encoded blocks $\boldsymbol{X}\left[n_{c}, k\right]$ are related to the STSK codewords by a one-to-one mapping relationship, the ML-MSDD in turn estimates the $\left(N_{w}-1\right)$ STSK codewords $S\left[n_{c}, k-N_{w}+2\right]$, 
$\ldots, \boldsymbol{S}\left[n_{c}, k\right]$, which further estimates the source information mapped to the STSK codewords.

For the sake of employing MSDD and in order to reduce the complexity associated with an exhaustive search, we employ ML-MSDSD similar to [145], [146] in order to search through the candidate set lying within a sphere of radius $\rho_{s}[69]$ :

$$
\sum_{\mu=1}^{N_{w}}\left\|\boldsymbol{Y}_{\mu, \mu}^{H} \mathcal{A}_{\mu}+\sum_{v=\mu+1}^{N_{w}}\left(\boldsymbol{Y}_{\mu, v}^{H} \mathcal{A}_{v}\right)\right\|^{2} \leq \rho_{s}^{2},
$$

where the accumulated differential matrices may be expressed as [148]

$$
\mathcal{A}_{v}= \begin{cases}\prod_{\lambda=v}^{N_{w}-1} \boldsymbol{S}_{\lambda}^{H}, & 1 \leqslant v \leqslant\left(N_{w}-1\right) \\ \boldsymbol{I}_{T}, & \boldsymbol{v}=N_{w}\end{cases}
$$

\section{B. Soft-Decision MAP-MSDSD Receiver}

The soft-MSDSD aided MC DSTSK scheme is portrayed in Fig. 22. Assuming the STSK codewords to be mutually independent, the sphere decoding technique of [149], the partial Euclidean distance (PED) criterion detailed in [145], [148], [150] may be invoked. Again, the MAP-MSDSD is initialized with $\mu=\left(N_{w}-1\right)$ and then proceeds by applying the abovementioned PED search criterion until we reach $\mu=1$, where the search radius is updated to $\rho^{2}=d_{1}^{2}$ and the search is repeated by commencing from $\mu=2$ until $\mu=\left(N_{w}-1\right)$ is reached. If the new search does not provide a better estimate, the previous estimate is retained.

Assuming a high interleaver depth so that the permuted bits may be treated as being independent, the a priori loglikelihood ratio (LLR) corresponding to the bit $b_{j}$ interleaved by the interleaver $\Pi_{2}$ of Fig. 22 is defined by [69], [148]: $L_{a}\left(b_{j}\right) \triangleq \ln \frac{P\left(b_{j}=b\right)}{P\left(b_{j}=\bar{b}\right)}$, where $b \in\{0,1\}$ and the $j$-th bit $b_{j}=b$ corresponds to the MAP-MSDSD estimate $\boldsymbol{S}^{\hat{w}}$, while $\bar{b}$ indicates its complement. The a posteriori LLR $L_{p}(\cdot)$ of $b_{j}$ may then be approximated by the maximum-logarithmic-MAP (max-logMAP) algorithm [69], [148]. The extrinsic LLR $L_{e}(\cdot)$ for $b_{j}$ is then evaluated by subtracting the a priori LLR $L_{a}(\cdot)$ from the a posteriori LLR $L_{p}(\cdot)$. The extrinsic information gleaned from the soft-MSDSD demapper is then iteratively exchanged with the URC decoder, which may be referred to as the inner iteration, while the exchange of extrinsic information between the URC and the RSC decoder of Fig. 22 may be referred to as the outer iteration. For each outer iteration between the RSC and the URC decoder, several inner iterations are invoked between the URC and the soft-MSDSD-aided DSTSK demapper [38], [69]. Finally, the bits are estimated from the a posteriori LLRs generated by the RSC decoder.

1) Complexity of the MAP-MSDSD-Aided DSTSK: In order to quantify the complexity of the MAP-MSDSD scheme, we may consider the number of real-valued multiplication operations (RMOs) required for obtaining a single soft-output value, which is used as the complexity metric. The lower bound of the complexity may be obtained [145], [148] as $[M N(2 M+$ 1) $\left./ \log _{2}(\mathcal{L} \cdot Q)+2 M^{2} N\right] N_{w}^{2}$ for large $N_{w}$.

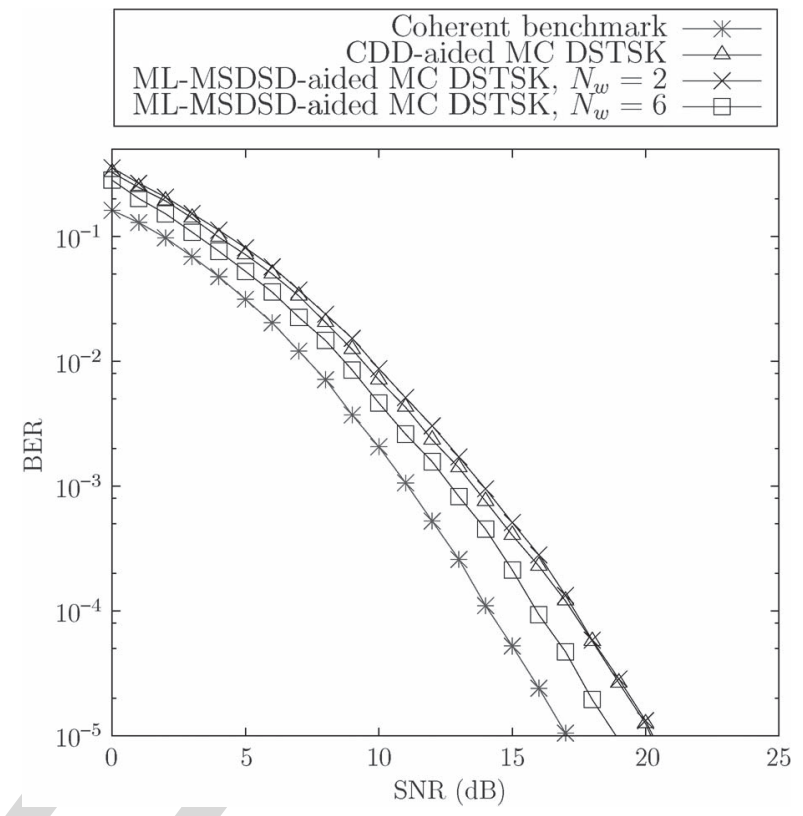

Fig. 23. Simulated BER performance of the ML-MSDSD aided QPSKmodulated MC DSTSK $(2,2,2,4)$ scheme for transmission over dispersive COST207-TU12 channel with normalized Doppler frequency $f_{d}=0.001$ and observation window sizes of $N_{w}=2$ and $N_{w}=6$. The difference in BER performance between coherent and noncoherent scheme decreases with increasing value of $N_{w}$.

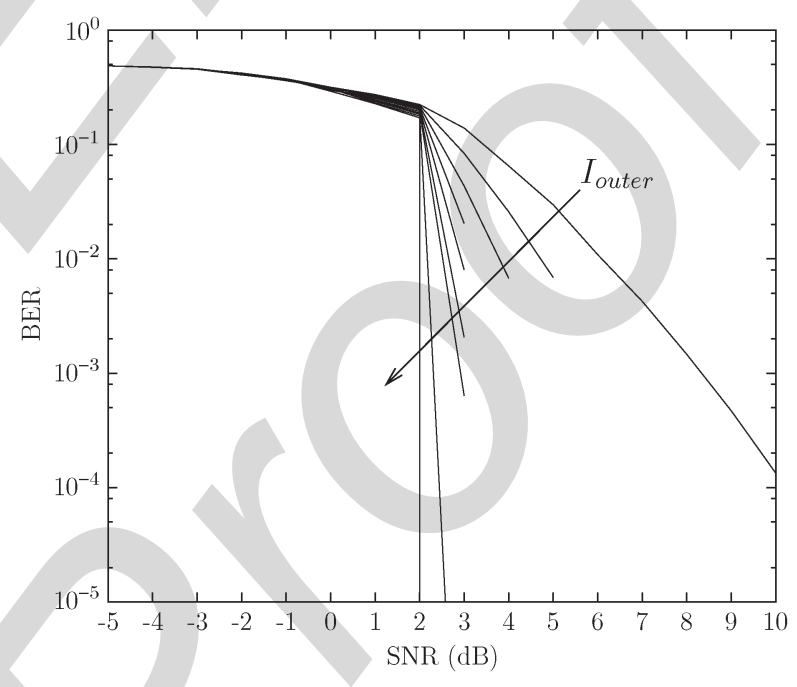

Fig. 24. Achievable BER performance of the soft-MSDSD-aided OFDM $\operatorname{DSTSK}(2,2,2,4)$ in conjunction with QPSK modulation and $N_{w}=4$ for transmission over dispersive COST207-TU12 channel using interleaver length of 200000 bits and a total of 11 outer iterations. Note that after $I_{\text {outer }}=$ 9 iterations, BER converge to vanishingly low BER and the curves are seen almost overlapping after $I_{\text {outer }}=9$ iterations.

2) Performance of the ML-MSDSD and MAP-MSDSDAided DSTSK: Fig. 23 characterizes the achievable BER of the ML-MSDSD-aided QPSK-modulated MC DSTSK $(2,2,2,4)$ scheme, while that of the soft-MSDSD-aided MC DSTSK scheme associated with different observation window sizes of $N_{w}=2,4,6,10$ is investigated in Figs. 24-26. We have employed the COST207-TU12 channel model for the links between each transmit-receive antenna pair and an $\operatorname{RSC}(2,1$, 2) outer code having octrally represented generator polynomials of $\left(g_{r}, g\right)=(3,2)_{8}$ as well as two random interleavers of length 


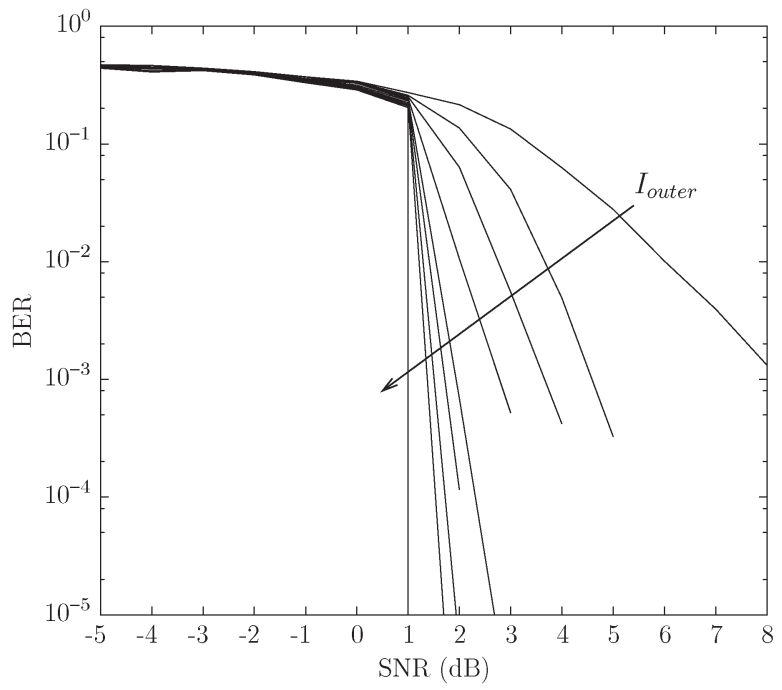

Fig. 25. Achievable BER performance of the soft-MSDSD-aided OFDM DSTSK $(2,2,2,4)$ in conjunction with QPSK modulation and $N_{w}=10$ for transmission over dispersive COST207-TU12 channel using interleaver length of 200000 bits and a total of 11 outer iterations. Note that after $I_{\text {outer }}=$ 9 iterations, BER converge to vanishingly low BER and the curves are seen almost overlapping after $I_{\text {outer }}=9$ iterations.

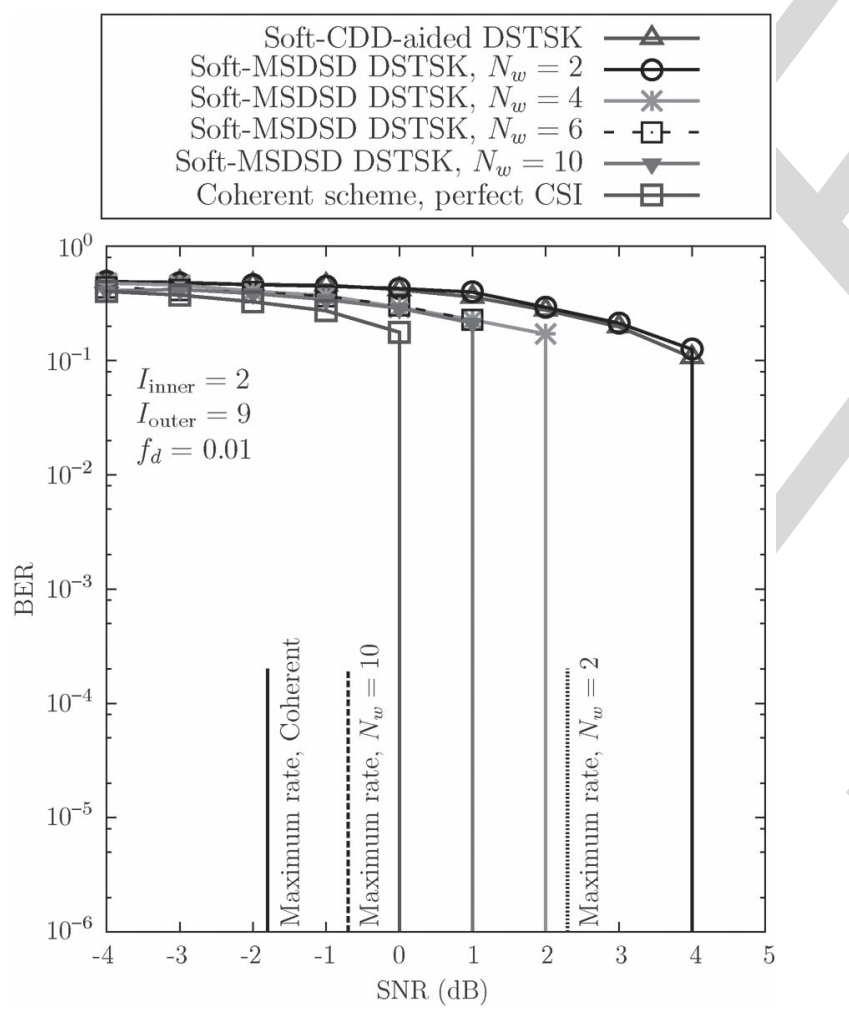

Fig. 26. Simulated BER performance of the soft-MSDSD aided QPSKmodulated MC DSTSK $(2,2,2,4)$ scheme [69] for transmission over dispersive COST207-TU12 channel with normalized Doppler frequency $f_{d}=0.001$ and different observation window size $N_{w}=2,4,6,10$. The BER falls sharply after $I_{\text {outer }}=9$ outer iterations as a benefit of employing the URC and the performance approaches that of the coherent scheme with perfect CSI with increasing value of $N_{w}$.

200000 bits. The number of inner and outer iterations was set to $I_{\text {inner }}=2$ and $I_{\text {outer }}=11$ respectively. As seen in Fig. 23, upon increasing the window size $N_{w}$, the BER performance difference between the coherent and noncoherent scheme is reduced. Figs. 24 and 25 were recorded for the soft-MSDSDaided OFDM DSTSK $(2,2,2,4)$ scheme associated with QPSK modulation and $N_{w}=4$ and $N_{w}=10$, respectively. Observe that the characteristics converge to a low BER for $I_{\text {outer }}=$ 9 iterations. For more than $I_{\text {outer }}=9$ iterations the curves become overlapped. The BER of the ML-MSDSD and softMSDSD-aided MC DSTSK schemes is compared furthermore to that of the corresponding coherent scheme relying on perfect CSI in Figs. 23 and 26. The MC DSTSK scheme has the benefit of dispensing with CE due to differential encoding, while mitigating the dispersion-induced performance erosion of classic STSK by employing OFDM. Furthermore, the multiple-symbol detection partially mitigates the inherent performance penalty imposed by noncoherent detection. Observe in Fig. 26 that as $N_{w}$ increases, the BER performance gradually approaches that of the perfect CSI-oriented coherent scheme. Note that all the performance characteristics converge to a vanishingly low BER after $I_{\text {outer }}=9$ iterations, which is the explicit benefit of using the URC in the system.

\section{Summary And Potential Future Research}

In this section, we summarize the philosophy of MC STSK schemes and the relevant design guidelines, followed by a range of research topics concerning potential future studies.

\section{A. Summary}

We have provided detailed transceiver designs for the family of STSK-based MIMO-MC systems for transmissions over dispersive wireless channels. More specifically, OFDM, MCCDMA, OFDMA and SC-FDMA aided MIMO systems employing the STSK concept were discussed. Furthermore, the successive DF relaying aided cooperative MC STSK scheme of [14] was conceived for eliminating the effects of correlated fading often experienced in non-cooperative scenarios, while mitigating the typical throughput loss of classic half-duplex relaying schemes. Furthermore, an OFDM-aided DSTSK arrangement of Fig. 22 was studied for the sake of dispensing with $\mathrm{CE}$, while simultaneously reducing the decoding complexity imposed.

Let us now compare the OFDM-aided coherent STSK (CSTSK) scheme of [11], the MC-CDMA-aided CSTSK regime of [12], the OFDMA/SC-FDMA-aided CSTSK arrangement of [13], the MC-CDMA-aided cooperative CSTSK and DSTSK architecture of [14] and the soft-MSDSD-aided MC DSTSK scheme of [145] in Table VII. We note that all these schemes mitigate the performance degradation imposed by the channel-induced dispersion, but as a further benefit, the MC-CDMA-aided STSK and the SC-FDMA-aided STSK are capable of providing additional FD diversity gains. Under the assumption of perfect orthogonality amongst the subcarriers, the SC-FDMA-aided scheme remains free from MUI, whereas the MUI imposed on the MC-CDMA-aided scheme can only be mitigated by a suitable MUD. The complexity associated with the employment of a MUD is typically high.

The decoding complexity of the different schemes highlighted in this treatise is summarized in Table VIII. In addition 
TABLE VII

Comparative ANALYSis of THE OFDM-Aided CSTSK SCHEME, THE MC-CDMA-AIDED CSTSK SCHEME, THE OFDMA/SC-FDMA-AIDED CSTSK SCHEME, THE MC-CDMA-AIDED COOPERATIVE CSTSK AND DSTSK SCHEMES AND SOFT-MSDSD-AIDED MC DSTSK SCHEME FOR TRANSMISSIONS OVER THE DiSPERSIVE COST 207-TU12 ChANNEL. Co-LoCATED STSK $(2,2,2,4)$, QPSK, AND Distributed STSK $(2,2,4)$, QPSK SCHEMES ARE CONSIDERED

\begin{tabular}{|c|c|c|c|c|c|c|}
\hline & $\begin{array}{r}\text { OFDM- } \\
\text { aided } \\
\text { CSTSK }\end{array}$ & $\begin{array}{r}\text { MC-CDMA- } \\
\text { aided } \\
\text { CSTSK }\end{array}$ & $\begin{array}{r}\text { OFDMA/SC-FDMA-aided } \\
\text { CSTSK }\end{array}$ & $\begin{array}{r}\text { Distributed } \\
\mathrm{MC} \\
\mathrm{CSTSK} \\
\end{array}$ & $\begin{array}{r}\text { Distributed } \\
\mathrm{MC} \\
\text { DSTSK } \\
\end{array}$ & $\begin{array}{r}\text { MSDSD- } \\
\text { aided MC } \\
\text { DSTSK }\end{array}$ \\
\hline $\begin{array}{l}\text { Schematic of } \\
\text { the scheme }\end{array}$ & Fig. 8 & Fig. 11 & $\begin{array}{r}\text { Fig. } 15 \text { and } 16 ; \\
\text { Fig. } 17 \\
\text { (Reduced-complexity detector) }\end{array}$ & $\begin{array}{r}\text { Fig. } 18 \text { and } \\
\text { Fig. } 19\end{array}$ & Fig. 21 & Fig. 22 \\
\hline CSI required? & yes & yes & yes & yes & no & no \\
\hline Detector & $\begin{array}{r}\text { Single- } \\
\text { stream } \\
\mathrm{ML} \\
\text { detector of } \\
\text { (13) }\end{array}$ & $\begin{array}{l}\text { SUD of (20) } \\
\& \text { ML-MUD } \\
\text { of [12] }\end{array}$ & $\begin{array}{r}\text { Single-stream ML detector of } \\
\text { (21) and reduced-complexity } \\
\text { detector of Algorithm } 1 \text { : }\end{array}$ & $\begin{array}{r}\text { Joint ML } \\
\text { detector of } \\
(21)\end{array}$ & $\begin{array}{r}\text { Joint ML } \\
\text { detector of } \\
(24), \text { but } \\
\text { for } \\
\text { differential } \\
\text { scheme } \\
\text { of [14] }\end{array}$ & $\begin{array}{r}\text { ML- } \\
\text { MSDSD } \\
\text { of (28) and } \\
\text { Soft- } \\
\text { decision } \\
\text { MAP- } \\
\text { MSDSD of } \\
\text { Sec- } \\
\text { tion VI-B }\end{array}$ \\
\hline $\begin{array}{l}\text { SNR at } \\
\text { BER }=10^{-5} \\
\text { (uncoded } \\
\text { scheme) }\end{array}$ & $16 \mathrm{~dB}$ & $\begin{array}{r}16 \mathrm{~dB} \\
\left(S_{f}=1\right) \\
13.5 \mathrm{~dB} \\
\left(S_{f}=4\right)\end{array}$ & $\begin{array}{r}16 \mathrm{~dB} \text { (OFDMA, ML) } \\
13.5 \mathrm{~dB} \text { (SC-FDMA, MMSE) }\end{array}$ & $\begin{array}{r}16 \mathrm{~dB} \\
(N=1)\end{array}$ & $\begin{array}{r}19 \mathrm{~dB} \\
(N=1)\end{array}$ & $\begin{array}{r}18.0 \mathrm{~dB} \\
\left(N_{w}=6\right)\end{array}$ \\
\hline
\end{tabular}

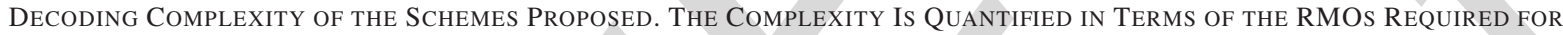
Estimating a Bit or for Obtaining a Single Soft-Output Value of the Soft-MSDSD-Aided MC DSTSK Scheme

\begin{tabular}{|l|r|}
\hline Decoding Scheme & Complexity \\
\hline \hline ML detector of (13) for OFDM-aided CSTSK & $\frac{4 M N T^{2} Q+6 N T Q \mathcal{L}}{\log _{2}(Q \cdot \mathcal{L})}$ \\
\hline The detector of (20) for MC-CDMA-aided CSTSK & $\frac{10 M T Q \mathcal{L}}{\log _{2}(Q \cdot \mathcal{L})}$ \\
\hline ML-MUD of [12] for MC-CDMA-aided CSTSK & $\frac{S_{f}\left(4 M N T^{2} Q U+5 N T Q \mathcal{L} U+2 N T Q \mathcal{L}\right)}{\log _{2}(Q \cdot \mathcal{L})}$ \\
\hline ML detector of [13] for SC-FDMA-aided CSTSK & $\frac{10 M T Q \mathcal{L}}{\log _{2}(Q \cdot \mathcal{L})}$ \\
\hline $\begin{array}{l}\text { Reduced-complexity detector of Algorithm 1: for } \\
\text { OFDMA/SC-FDMA-aided CSTSK }\end{array}$ & $\frac{4 M^{2} N+8 M N+4 M T Q+2 Q \mathcal{L}^{1}+Q+2 \mathcal{L}}{\log _{2}(Q \cdot \mathcal{L})}$ \\
\hline $\begin{array}{l}\text { Joint ML detector of [14] } \\
\text { MAP-MSDSD of Section VI-B for soft-MSDSD-aided } \\
\text { DSTSK }\end{array}$ & $\geq\left[M N(2 M+1) / \log _{2}(\mathcal{L} \cdot Q)+2 M^{2} N\right] N_{w}^{2}$ \\
\hline
\end{tabular}

to the low decoding complexity imposed by the SC-FDMAaided scheme, it has the additional benefit of providing a low PAPR, and hence it is particularly suitable for uplink transmissions. It is also worth noting that the distributed MC STSK scheme of [14] has been investigated in the context of a single DN, but these studies may be readily extended to multiple DNs.

\section{B. Design Guidelines}

The design guidelines of the MC STSK schemes relying on the $(M, N, T, Q)$ parameters in conjunction with the $\mathcal{L}$-ary constellation of the STSK system as a function of the number of subcarriers $N_{c}$, the spreading factor $S_{f}$, the DFT-spreading length $N_{d}$ and the CP-length $L_{\mathrm{cp}}$ are summarized as follows:

1) The values of $(M, N)$ specify the number of transmit and receive AEs.

2) The values of $(Q, \mathcal{L}, T)$, i.e., the number of DMs, the modulation order and the number of symbol intervals over which spreading takes place are chosen in any space-time block so as to satisfy the desirable DMT characterized by the rate $R=\log _{2}(\mathcal{L} \cdot Q) / T$ and by the achievable diversity order of $N \cdot \min (M, N)$.

3) For all the possible combinations of $(\mathcal{L}, Q)$, the $Q \mathrm{DMs}$ are computed based on either the DCMC capacityoptimal or the PEP-optimal design procedure [5], [10]. 
4) The best set of $(\mathcal{L}, Q)$ may be found by predicting the BER performance with the aid of the corresponding EXIT charts.

5) The number of subcarriers is chosen to ensure that the codeword symbols of each subcarrier experience frequency-flat fading. To be specific, the MC modulation scheme is designed by ensuring that the coherence bandwidth of the channel is higher than the subcarrier symbol rate. Additionally, the length of the $\mathrm{CP}$ adopted ensures the absence of ISI between the consecutive OFDM symbols.

6) In the MC-CDMA aided STSK scheme, the number of subcarriers $N_{c}$ has to be equal to or a multiple of the spreading factor $S_{f}$, where increasing $S_{f}$ provides a higher FD diversity gain.

7) In the OFDMA/SC-FDMA aided STSK arrangement, the number of subcarriers $N_{c}$ is usually a multiple of the DFTspreading length $N_{d}$, whereas for a fully-loaded system supporting $U$ users, we have $N_{c}=\left(N_{d} \times U\right)$.

8) In the coherent SR aided cooperative MC-STSK arrangement, the parameters used are designed so as to support the date rate of $R=\frac{2 \log _{2}(\mathcal{L} \cdot Q)}{b+T}$ [bits/symbol duration], where $b$ represents the number of symbols in the SN's transmit blocks.

9) The differential cooperative MC STSK and the softMSDSD aided MC-STSK are designed using directly generated unitary matrices, while satisfying the relationship of $M=T$.

\section{Future Research}

In this section, we briefly discuss a range of potential future research ideas.

1) Asynchronous Transmission: A major challenge of MIMO systems is the IAS problem. The synchronization issue becomes acute in cooperative scenarios due to the asynchronous nature of relay nodes in the network. Perfect synchronization was considered in schemes discussed, although in practice it is difficult to acquire accurate system-level synchronization without imposing a high additional synchronization overhead and implementation complexity. The investigations of [151] revealed that the time-synchronization errors encountered in a cooperative scenario impose a significant performance degradation. Additionally, the propagation delays arising both from the different node locations in a cooperative scheme and from the multipath-induced delay-spread contribute to the sampling offset errors, which further aggravates the effect of synchronization errors. For mitigating the performance degradation imposed by synchronization errors in a cooperative regime, a number of space-time codes were proposed in [152], [153], which invoke space-time equalization under the assumption that the perfect knowledge of the CSI and/or of the relative delays is available at the receiver. In [154], [155], multi-carrier aided STCs were proposed specifically for dispersive links. However, all these schemes suffer from a high computational complexity.

In order to dispense with system-level synchronization between the RF chains associated with the transmit AEs, asynchronous STSK (ASTSK) was proposed in [8], [10]. This scheme operates in a way similar to the SM/SSK systems, because a single $\mathrm{AE}$ is activated at each symbol duration, whilst maintaining the benefits of the STSK system. However, the ASTSK scheme imposes a further constraint on the design of DMs, as discussed in Section II-C1. More specifically, the ASTSK DMs must not contain more than a single non-zero element in each column. Indeed, obtaining suitable DMs which are capable of meeting the required capacity and/or BER performance targets, while satisfying the power constraint mentioned in (5), is a challenging task. The design of a beneficial asynchronous MC STSK arrangement in the context of large-scale (LS) MIMOs [156]-[159] constitutes a set of major open challenges for future research. More specifically, like the SM/SSK schemes, ASTSK has the potential of low-cost hardware design in the context of LS-MIMO systems [70], [160]. Furthermore, the impact of synchronization errors imposed on the cooperative regime of Figs. 20 and 21 and the corresponding design alternative conceived for the asynchronous transmissions has to be investigated.

2) Multiuser Multicell Scenario: The MC STSK scheme relying on both co-located as well as on distributed AEs may be further investigated for employment in multiuser, multicell systems. The scheme may be employed in a coordinated multipoint (CoMP) communications scenario, where the different base stations (BSs) operate in a coordinated manner in order to mitigate the intercell interference and to increase the cell edge throughput [161]-[164]. The scheme may also be considered for employment both in heterogeneous networks (HetNets) including femto/pico BSs, fixed/mobile relays and cognitive radios as well as in appropriate interference mitigation arrangements. The concept of area spectral efficiency (ASE) was conceived in [165] for quantifying the spectral efficiency per unit area of a cellular mobile radio system. In [61], the ASE of soft-decision space-time-frequency-shift keying (STFSK) aided slow-frequency-hopping multiple access (SFHMA) was investigated. Designing appropriate interference management for the schemes of Figs. 20 and 21 under a multiuser multicell scenario constitutes an open problem. A holistically optimized system incorporating the MC STSK scheme may be considered for providing a high ASE.

3) Coded Modulation Aided MC STSK Scheme: The MC STSK scheme may be amalgamated with the family of classic coded modulation schemes, such as Ungerboeck's trellis-coded modulation (TCM) [166]-[168], turbo trellis-coded modulation of Robertson and Worz (TTCM) [169], the bit-interleaved coded modulation (BICM) of Caire et al. [170] and the bit-interleaved coded modulation regime of $\mathrm{Li}$ et al. [171] relying on iterative decoding (BICM-ID). The bandwidthefficient BICM scheme has been widely studied, for example in [172]-[174]. We have employed a near-capacity three-stage coding arrangement for the MC STSK schemes of Fig. 22 and of [12], [13]. These three-stage coding architectures are essentially similar to the BICM technique. However, a comprehensive study of the coded modulation aided MC STSK scheme's performance constitutes a major item of potential future study.

4) Network Coding for Cooperative MC STSK Scheme: Network coding is a promising technique for wireless 
communication systems, which was conceived in the seminal paper by Ahlswede et al. [175] and it is especially beneficial for the sake of increasing the attainable network throughput, the scalability and robustness. Rather than simply replicating and relaying the SN's transmissions at the RNs, the signals are recombined at the intermediate nodes for example by their "Ex-OR" operation or by other linear combination techniques, which are then retransmitted [176], [177]. This was shown to be particularly beneficial in terms of approaching the maximum achievable information flow of the network. Network coding has the potential of improving the robustness, while simultaneously increasing the attainable throughput and reducing the delay of the system. Network coding [178] may be employed in the cooperative MC STSK scheme for further enhancing the ASE. Specifically, the cooperative coherent MC STSK scheme of [14] with its VAAs shown in Fig. 20 and the cooperative MC DSTSK scheme of Fig. 21 may provide increased throughput with the aid of incorporating network coding. However, physical layer network coding and the associated recombining operation at the VAAs would not permit employment of CRC. This is because in a network coded scenario the VAAs will recombine the SN to VAA signal and the inter-VAA interference impinging on them either in the previous or in the next signaling period.

5) Subcarrier Allocation and Scheduling of MC STSK Schemes: We have also the further motivation to design joint power- and sub-carrier allocation schemes for the OFDMA/SCFDMA based STSK DL/UL scenarios. There are numerous popular subcarrier allocation algorithms, such as the Greedy algorithm of Kim and Kim [179], the Hungarian algorithm of Harold [180] and the Worst Subcarrier Avoidance (WSA) technique [181] of Ermolova and Makarevitch, etc. Hence the performance of efficient power and sub-carrier allocation techniques has to be investigated in the context of MC STSK schemes, such as the one proposed by Liu and Yang for lowcomplexity subcarrier-power allocation in [182]. When considering the multiuser, multicell scenarios, appropriate upper layer concepts may be conceived for providing multiuser diversity. Specifically, channel-quality dependent multi-carrier as well as multiuser scheduling has to be invoked for allocating both the spectral and the power resources to different users for the sake of maximizing a meaningful $\mathrm{OF}$, such as for example the total throughput [183]. The performance of different scheduling algorithms also has to be compared. In [184], [185], the authors designed opportunistic relay selection schemes for the SCFDMA uplink. The performance of sophisticated relay selection schemes has to be studied in the context of cooperative multi-carrier STSK.

6) Energy-Efficient MC STSK Design: Against the backdrop of rising energy cost and carbon footprint of cellular networks, "green" wireless systems designed for a high throughput, whilst maintaining a high Quality-of-Service (QoS) and flexible scalability represent a few of the compelling needs of future communication systems. This necessitates the development of power-efficient, low-complexity solutions, whilst satisfying the target QoS as well as the throughput requirements [70], [186]. An appropriate design metric to be invoked may be the normalized energy-efficiency (EE) of Li et al. [187] expressed as the throughput per unit energy. The MC STSK schemes studied in this paper have the potential of considerably reducing the power consumption of wireless systems as a benefit of its inherent diversity-multiplexing gain trade-off. The schemes activate a single DM at any symbol instant and the DMs may be designed for minimizing the overall energy consumption/dissipation. Furthermore, cross-layer-aware upper layer schemes may be designed for supporting energy-efficient operation of the MC STSK schemes.

7) Potential Impact of MC STSK on 4G/5G Wireless-LSMIMO and Millimeter Wave Communications: To cope with the high demand for data services over the Internet, wireless standards are being developed at a breathtaking speed. These standards incorporate all significant improvements in the field of wireless access techniques. For example, wideband code division multiple access (WCDMA) and adaptive modulation and coding (AMC) aided high speed packet access (HSPA) were incorporated in the $3 \mathrm{G}$ specifications. The $4 \mathrm{G}$ concept of the International Mobile Telecommunications-Advanced (IMTAdvanced) system incorporates MIMOs for achieving beneficial multiplexing, diversity and adaptive beamforming gains amalgamated with a full-fledged OFDM and OFDMA-based radio access technology [188], [189]. The 5G system is expected to be rolled out by 2020 for facilitating ubiquitous and seamless connectivity by incorporating techniques such as LS-MIMOs [160], [190], CoMP [162], [191], device-to-device (D2D) communications [192]-[194], energy-efficient systems [195], [196] and millimeter wave (mm-wave) communications [189], [197]. Compared to 4G networks, 5G networks are expected to achieve 1000 times the system capacity and 10 times the spectral efficiency and data rate [196]. This may be achievable with the aid of LS-MIMOs in conjunction with interference-aware multi-tier networks [198], [199] comprising macro cells as well as small cells, relays and D2D networks. Each user of an LS-MIMO system is capable of scaling down its transmit power proportionally to the number of BS antennas [200], thereby providing an improved EE. LS-MIMO systems relying on adaptive beamforming are also compatible with the emerging technique of exploring the underutilized mm-wave frequency spectrum [189], [190], [201], [202]. Rappaport et al. [189] conducted extensive propagation measurements both at $28 \mathrm{GHz}$ as well as at $38 \mathrm{GHz}$ and advocated the inclusion of the unlicensed $\mathrm{mm}$-wave bands in the $5 \mathrm{G}$ cellular landscape.

LS-MIMOs are particularly suitable for the SM scheme, since a high multiplexing gain may be attained at a reduced complexity due to the employment of a limited number of RF chains. However, the practical implementation of SM faces the challenge of acquiring CSI both at the transmitter and at the receiver [70]. The problem of acquiring CSI at the receiver might become acute, when transmit beamforming will be required at the BS. As an impediment, the SM scheme does not provide transmit diversity. By contrast, as a benefit, the OFDMA aided STSK DL and the SC-FDMA aided STSK UL of Section IV-C is readily compatible with the LTE-Advanced physical layer specifications. The MC STSK schemes studied in this treatise also have the potential of supporting LS-MIMO-based 5G networks. The schemes impose low decoding complexity as a benefit of employing single-stream transmission. Hence the number of RF chains may be adaptively selected by a suitable 
design of the STSK DMs. To be more specific, each of the $Q$ DMs, $\boldsymbol{A}_{q},(q=1,2, \ldots, Q)$ may be constructed for ensuring that there is only a low number of non-zero elements in each column of the DMs. This additional constraint imposed on the DM optimization procedure may assist us in using a limited number of RF chains, thereby improving the EE, whilst still retaining the benefits of the STSK scheme. This additional constraint, however, considerably reduces the search space of the set of DMs. The design of efficient DMs for attaining the desired DMT-especially in an LS-MIMO scenario-constitutes an open challenge.

Again, mm-wave techniques constitute promising solutions as part of the HetNet 5G landscape. Due to the decrease in wavelength at these high frequencies, a huge number of halfwavelength-spaced beamforming elements can be accommodated in a limited space, especially at the BS. This renders the MC STSK-based LS-MIMOs a viable solution to attain a flexible DMT at a low complexity and at a low power consumption. In practice, however, mm-wave cellular systems have to compensate for the high free-space path loss. Hence, $5 \mathrm{G}$ cellular systems have to take advantage of the high-dimensional beamforming-aided directional antenna arrays [70], [197], [203], [204]. In the light of this impediment imposed by the use of mm-wave frequencies, the application of MC STSK schemes at these frequencies necessitates practical solutions, which are capable of simultaneously providing diversity, multiplexing and beamforming gains. To the best of our knowledge, the application of MC STSK schemes to the mm-wave band of frequencies has not yet been investigated.

\section{CONCLUSION}

Having commenced with a brief review of MIMO-MC systems and with an introductory discourse on the development of the beneficial STSK system, we provide helpful guidelines for designing MC STSK schemes in this tutorial. Both single-user versus multiuser as well as coherent versus noncoherent $\mathrm{MC}$ STSK were reviewed in this paper. The schemes highlighted here are beneficial in terms of striking a trade-off between the achievable diversity and multiplexing gains, as well as between the performance attained and the complexity imposed, while facilitating communications over dispersive wireless channels. In a nutshell, the basic motivation of the above-mentioned designs was that of improving the overall integrity and throughput of wireless systems by striking a compelling compromise among the conflicting design factors, such as the effective throughput, the coding as well as diversity gain, the implementation complexity etc. To outline the rationale of our work and to summarize our main contributions, we conclude as follows:

- STSK has the benefit of striking a flexible DMT by using single-stream ML detection, as mentioned for example in [8]-[10]. However, all the previous studies apart from [54] investigated STSK in non-dispersive scenarios.

- In order to retain all the benefits of STSK for transmission over dispersive channels, we need either a space-time equalizer or OFDM/MC-CDMA. The latter converts a dispersive channel into reduced-rate parallel non-dispersive channels [11], [12].

- To maintain the benefits of OFDM-based transmission, while facilitating multiuser communications, OFDMA/ SC-FDMA aided STSK systems [13] were proposed. In order to further mitigate the decoding complexity of the single-stream ML detector, a reduced-complexity detector was proposed in [137].

- The transmit power may be reduced with the aid of relaying, especially by DF relaying, where the plausible benefit is that in the absence of decoding errors, the relay retransmits to the DN from a reduced distance.

- Albeit relaying is highly beneficial, it requires both a broadcast and a cooperation slot, which potentially halves the achievable throughput. This disadvantage may be mitigated with the aid of SR, which is achieved at the cost of reducing the number of users supported. Hence, a SRaided coherent versus noncoherent cooperative MC STSK arrangement was studied in [14]. To be specific, the pros and cons of coherent versus noncoherent detection were quantified. Explicitly, in practical terms, it is more realistic to use noncoherent detection dispensing with $\mathrm{CE}$ at the relay nodes.

- Differential STSK employing both hard-decision and softdecision MSDSD were studied for the sake of dispensing with $\mathrm{CE}$ and thus mitigating the potentially high Dopplerdependent pilot overhead, while benefiting both from the low complexity imposed by sphere decoding and from the iterative exchange of soft-information during detection.

\section{Acronyms}

$\mathrm{AE}$

AMC

ASE

ASTSK

BER

BICM

BICM-ID

BLAST

CCMC

CDD

CE

CIR

CoMP

CRC

CSI

CSTSK

D2D

DAB

DCMC

DF

DFT

DL

$\mathrm{DM}$
Antenna Element.

Adaptive Modulation and Coding.

Area Spectral Efficiency.

Asynchronous Space-Time Shift Keying.

Bit Error Ratio.

Bit-interleaved Coded Modulation.

Bit-interleaved Coded Modulation with Iterative Decoding.

Bell Laboratories Layered Space-Time.

Continuous input Continuous output Memory-

less Channel.

Conventional Differential Detection.

Channel Estimation.

Channel Impulse Response.

Coordinated Multipoint.

Cyclic Redundancy Check.

Channel State Information.

Coherent Space-Time Shift Keying.

Device-to-device.

Digital Audio Broadcasting.

Discrete input Continuous output Memoryless Channel.

Decode-and-Forward.

Discrete Fourier Transform.

Downlink.

Dispersion Matrix. 


$\begin{array}{lll}\text { DMT } & \text { Diversity-Multiplexing Tradeoff. } & \text { SUD } \\ \text { DN } & \text { Destination Node. } & \text { TA } \\ \text { DSFM } & \text { Differential Space-Frequency Modulation. } & \text { TCM } \\ \text { DSTSK } & \text { Differential Space-Time Shift Keying. } & \text { TD } \\ \text { DTDR } & \text { Distributed Transmit Directional Receive. } & \text { TTCM } \\ \text { DVB } & \text { Digital Video Broadcasting. } & \text { TU } \\ \text { EE } & \text { Energy Efficiency. } & \text { UL } \\ \text { EGC } & \text { Equal Gain Combining. } & \text { V-BLAST } \\ \text { FD } & \text { Frequency Domain. } & \text { VAA } \\ \text { GA } & \text { Genetic Algorithm. } & \text { WCDMA } \\ \text { HetNet } & \text { Heterogeneous network. } & \text { WINNER } \\ \text { HSPA } & \text { High-Speed Packet Access. } & \text { WSA } \\ \text { IAS } & \text { Inter-Antenna Synchronization. } & \end{array}$

IDFT Inverse Discrete Fourier Transform.

IFDMA Inteleaved Frequency Division Multiple Access. LFDMA Localized Frequency Division Multiple Access. LLR Logarithmic Likelihood Ratio.

LS Large-Scale.

LTE Long Term Evolution.

MAP Maximum a posteriori.

MC Multicarrier.

MC-CDMA Multicarrier Code Division Multiple Access.

MIMO Multiple-Input Multiple-Output.

ML Maximum Likelihood.

MRC Maximal Ratio Combining.

MS Mobile Station.

MSDD Multiple-Symbol Differential Decoding.

MSDSD Multiple-Symbol Differential Sphere Decoding.

MT-CDMA Multitone Code Division Multiple Access.

MUD Multiuser Detector.

MUI Multiuser Interference.

OF Objective Function.

OFDM Orthogonal Frequency Division Multiplexing.

OFDMA Orthogonal Frequency Division Multiple

Access.

$\begin{array}{ll}\text { ORC } & \text { Orthogonality Restoring Combining. } \\ \text { PAPR } & \text { Peak-to-Average Power Ratio. } \\ \text { PEP } & \text { Pairwise Error Probability. } \\ \text { QoS } & \text { Quality-of-Service. } \\ \text { RF } & \text { Radio Frequency. } \\ \text { RMO } & \text { Real-valued Multiplication Operation. } \\ \text { RN } & \text { Relay Node. } \\ \text { SC } & \text { Selection Combining. } \\ \text { SC-FDMA } & \text { Single-Carrier Frequency Division Mult } \\ & \text { Access. } \\ \text { SCM } & \text { Single-Carrier Modulation. } \\ \text { SD } & \text { Spatial Domain. } \\ \text { SDM } & \text { Spatial Division Multiplexing. } \\ \text { SDMA } & \text { Space-Division Multiple Access. } \\ \text { SFHMA } & \text { Slow-Frequency-Hopping Multiple Access. } \\ \text { SM } & \text { Spatial Modulation. } \\ \text { SN } & \text { Source Node. } \\ \text { SR } & \text { Successive Relaying. } \\ \text { SSK } & \text { Space Shift Keying. } \\ \text { STBC } & \text { Space-Time Block Code. } \\ \text { STFSK } & \text { Space-Time-Frequency Shift Keying. } \\ \text { STSK } & \text { Space-Time Shift Keying. } \\ \text { STTC } & \text { Space-Time Trellis Code. }\end{array}$

Single-User Detection.

Transmit Antenna.

Trellis-Coded Modulation.

Time Domain.

Turbo Trellis-Coded Modulation.

Typical Urban.

Uplink.

Vertical Bell Laboratories Layered Space-Time.

Virtual Antenna Array.

Wideband Code Division Multiple Access.

Wireless INitiative NEw Radio.

Worst Subcarrier Avoidance.

\section{REFERENCES}

[1] A. Goldsmith, S. A. Jafar, N. Jindal, and S. Vishwanath, "Capacity limits of MIMO channels," IEEE J. Sel. Areas Commun., vol. 21, no. 5, pp. 684-702, Jun. 2003

[2] E. Telatar, "Capacity of multi-antenna Gaussian channels," Eur. Trans. Telecommun., vol. 10, no. 6, pp. 585-595, Nov./Dec. 1999.

[3] L. Zheng and D. N. C. Tse, "Diversity and multiplexing: A fundamental tradeoff in multiple-antenna channels," IEEE Trans. Inf. Theory, vol. 49, no. 5, pp. 1073-1096, May 2003.

[4] B. Hassibi and B. M. Hochwald, "High-rate codes that are linear in space and time," IEEE Trans. Inf. Theory, vol. 48, no. 7, pp. 1804-1824, Jul. 2002.

[5] R. W. Heath, Jr. and A. J. Paulraj, "Linear dispersion codes for MIMO systems based on frame theory," IEEE Trans. Signal Process., vol. 50, no. 10, pp. 2429-2441, Oct. 2002.

[6] R. Mesleh, H. Haas, S. Sinanovic, C. W. Ahn, and S. Yun, "Spatial modulation," IEEE Trans. Veh. Technol., vol. 57, no. 4, pp. 2228-2241, Jul. 2008.

[7] J. Jeganathan, A. Ghrayeb, L. Szczecinski, and A. Ceron, "Space shift keying modulation for MIMO channels," IEEE Trans. Wireless Commun., vol. 8, no. 7, pp. 3692-3703, Jul. 2009.

[8] S. Sugiura, S. Chen, and L. Hanzo, "Coherent and differential space-time shift keying: A dispersion matrix approach," IEEE Trans. Commun., vol. 58, no. 11, pp. 3219-3230, Nov. 2010.

[9] S. Sugiura, S. Chen, and L. Hanzo, "Generalized space-time shift keying designed for flexible diversity-, multiplexing- and complexity-tradeoffs," IEEE Trans. Wireless Commun., vol. 10, no. 4, pp. 1144-1153, Apr. 2011

[10] S. Sugiura, S. Chen, and L. Hanzo, "A universal space-time architecture for multiple-antenna aided systems," IEEE Commun. Surveys Tuts., vol. 14 , no. 2, pp. 401-420, 2012

[11] M. Driusso, F. Babich, M. I. Kadir, and L. Hanzo, "OFDM aided space-time shift keying for dispersive downlink channels," in Proc. IEEE VTC_Fall, Quebec City, QC, Canada, Sep. 2012, pp. 1-5.

[12] M. I. Kadir, S. Sugiura, S. Chen, and L. Hanzo, "MC-CDMA aided multi-user space-time shift keying in wideband channels," in Proc. IEEE WCNC, Shanghai, China, 2013, pp. 2643-2648.

[13] M. I. Kadir, S. Sugiura, J. Zhang, S. Chen, and L. Hanzo, "OFDMA/ SC-FDMA aided space-time shift keying for dispersive multiuser scenarios," IEEE Trans. Veh. Technol., vol. 62, no. 1, pp. 408-414, Jan. 2013

[14] M. I. Kadir, L. Li, S. Chen, and L. Hanzo, "Successive-relaying-aided decode-and-forward coherent versus noncoherent cooperative multicarrier space-time shift keying," IEEE Trans. Veh. Technol., vol. 62, no. 6, pp. 2544-2557, Jul. 2013.

[15] W. C. Jakes and D. C. Cox, Microwave Mobile Communications. New York, NY, USA: Wiley/IEEE Press, 1994.

[16] J. N. Laneman and G. W. Wornell, "Distributed space-time-coded protocols for exploiting cooperative diversity in wireless networks," IEEE Trans. Inf. Theory, vol. 49, no. 10, pp. 2415-2425, Oct. 2003.

[17] T. Wang, A. Cano, G. B. Giannakis, and J. N. Laneman, "Highperformance cooperative demodulation with decode-and-forward relays," IEEE Trans. Commun., vol. 55, no. 7, pp. 1427-1438, Jul. 2007.

[18] S. Sugiura, S. Chen, H. Haas, P. Grant, and L. Hanzo, "Coherent versus non-coherent decode-and-forward relaying aided cooperative space-time shift keying," IEEE Trans. Commun., vol. 59, no. 6, pp. 1707-1719, Jun. 2011. 
[19] Y. Fan, C. Wang, J. Thompson, and H. V. Poor, "Recovering multiplexing loss through successive relaying using repetition coding," IEEE Trans. Wireless Commun., vol. 6, no. 12, pp. 4484-4493, Dec. 2007.

[20] L. Kong, S. X. Ng, R. G. Maunder, and L. Hanzo, "Near-capacity cooperative space-time coding employing irregular design and successive relaying," IEEE Trans. Commun., vol. 58, no. 8, pp. 2232-2241, Aug. 2010.

[21] J. N. Laneman, D. N. C. Tse, and G. W. Wornell, "Cooperative diversity in wireless networks: Efficient protocols and outage behavior," IEEE Trans. Inf. Theory, vol. 50, no. 12, pp. 3062-3080, Dec. 2004.

[22] D. G. Brennan, "Linear diversity combining techniques," Proc. IEEE, vol. 91 , no. 2, pp. 331-356, Feb. 2003

[23] N. Seshadri and J. H. Winters, "Two signaling schemes for improving the error performance of Frequency Division Duplex (FDD) transmission systems using transmitter antenna diversity," Int. J. Wireless Inf. Netw., vol. 1, no. 1, pp. 49-60, Jan. 1994

[24] S. M. Alamouti, "A simple transmit diversity technique for wireless communications," IEEE J. Sel. Areas Commun., vol. 16, no. 8, pp. 14511458, Oct. 1998.

[25] V. Tarokh, H. Jafarkhani, and A. R. Calderbank, "Space-time block codes from orthogonal designs," IEEE Trans. Inf. Theory, vol. 45, no. 5, pp. 1456-1467, Jul. 1999.

[26] V. Tarokh, N. Seshadri, and A. R. Calderbank, "Space-time codes for high data rate wireless communication: Performance criterion and code construction," IEEE Trans. Inf. Theory, vol. 44, no. 2, pp. 744-765, Mar. 1998.

[27] B. L. Hughes, "Differential space-time modulation," IEEE Trans. Inf. Theory, vol. 46, no. 7, pp. 2567-2578, Nov. 2000.

[28] V. Tarokh and H. Jafarkhani, "A differential detection scheme for transmit diversity," IEEE J. Sel. Areas Commun., vol. 18, no. 7, pp. 11691174, Jul. 2000.

[29] A. J. Paulraj and T. Kailath, "Increasing capacity in wireless broadcast systems using Distributed Transmission/Directional Reception (DTDR),” U.S. Patent 5345 599, Sep. 6, 1994.

[30] G. J. Foschini, "Layered space-time architecture for wireless communication in a fading environment when using multiple antennas," Bell Labs. Tech. J., vol. 1, no. 2, pp. 41-59, 1996.

[31] G. J. Foschini, G. D. Golden, R. A. Valenzuela, and P. W. Wolniansky, "Simplified processing for high spectral efficiency wireless communication employing multi-element arrays," IEEE J. Sel. Areas Commun., vol. 17, no. 11, pp. 1841-1852, Nov. 1999

[32] J. H. Winters, J. Salz, and R. D. Gitlin, "The impact of antenna diversity on the capacity of wireless communication systems," IEEE Trans Commun., vol. 42, no. 2-4, pp. 1740-1751, Feb.-Apr. 1994.

[33] L. C. Godara, "Application of antenna arrays to mobile communications. Part I: Performance improvement, feasibility, system considerations," Proc. IEEE, vol. 85, no. 7, pp. 1031-1060, Jul. 1997.

[34] M. Cooper and M. Goldburg, "Intelligent antennas: Spatial division multiple access," in Proc. ArrayComm, Annu. Rev. Commun., 1996, pp. 999-1002.

[35] P. Vandenameele, L. V. D. Perre, M. G. E. Engels, B. Gyselinckx, and H. J. D. Man, "A combined OFDM/SDMA approach," IEEE J. Sel. Areas Commun., vol. 18, no. 11, pp. 2312-2321, Nov. 2000.

[36] H. Kim and J. Chun, "MIMO structure which combines the spatial multiplexing and beamforming," in Proc. IEEE VTC-Spring, Milan, Italy, May 2004, pp. 108-112.

[37] M. El-Hajjar, O. Alamri, J. Wang, S. Zummo, and L. Hanzo, "Layered steered space-time codes using multi-dimensional sphere packing modulation," IEEE Trans. Wireless Commun., vol. 8, no. 7, pp. 3335-3340, Jul. 2009.

[38] L. Hanzo, O. Alamri, M. El-Hajjar, and N. Wu, Near-Capacity MultiFunctional MIMO Systems (Sphere-Packing, Iterative Detection, and Cooperation). Hoboken, NJ, USA: Wiley, 2009.

[39] J. Mietzner, R. Schober, L. Lampe, W. H. Gerstacker, and P. A. Hoeher, "Multiple-antenna techniques for wireless communications-A comprehensive literature survey," IEEE Commun. Surveys Tuts., vol. 11, no. 2, pp. 87-105, 2009.

[40] M. Jiang and L. Hanzo, "Multiuser MIMO-OFDM for next-generation wireless systems," Proc. IEEE, vol. 95, no. 7, pp. 1430-1469, Jul. 2007.

[41] L. C. Godara, "Application of antenna arrays to mobile communications. Part II.: Beam-forming and direction-of-arrival considerations," Proc. IEEE, vol. 85, no. 8, pp. 1195-1245, Aug. 1997.

[42] J. H. Winters, "Smart antennas for wireless systems," IEEE Personal Commun., vol. 5, no. 1, pp. 23-27, Feb. 1998.
[43] L.-L. Yang and L. Hanzo, "Performance of generalized multicarrier DS-CDMA over Nakagami-m fading channels," IEEE Trans. Commun., vol. 50, no. 6, pp. 956-966, Jun. 2002.

[44] Y. A. Chau and S.-H. Yu, "Space modulation on wireless fading channels," in Proc. IEEE VTC-Fall, Atlantic City, NJ, USA, Oct. 2001, pp. 1668-1671.

[45] R. Mesleh, H. Haas, C. W. Ahn, and S. Yun, "Spatial modulationA new low complexity spectral efficiency enhancing technique," in Proc. CHINACOM, Beijing, China, Oct. 2006, pp. 1-5.

[46] R. Mesleh, S. Engelken, S. Sinanovic, and H. Haas, "Analytical SER calculation of spatial modulation," in Proc ISSSTA, Bologna, Italy, Aug. 2008, pp. 272-276.

[47] J. Jeganathan, A. Ghrayeb, and L. Szczecinski, "Spatial modulation: Optimal detection and performance analysis," IEEE Commun. Lett., vol. 12 , no. 8, pp. 545-547, Aug. 2008.

[48] S. Sugiura, C. Xu, S. X. Ng, and L. Hanzo, "Reduced-complexity coherent versus non-coherent QAM-aided space-time shift keying," IEEE Trans. Commun., vol. 59, no. 11, pp. 3090-3101, Nov. 2011.

[49] R. Mesleh, M. D. Renzo, H. Haas, and P. M. Grant, "Trellis coded spatial modulation," IEEE Trans. Wireless Commun., vol. 9, no. 7, pp. 23492361, Jul. 2010.

[50] M. D. Renzo and H. Haas, "Space Shift Keying (SSK-) MIMO over correlated Rician fading channels: Performance analysis and a new method for transmit-diversity," IEEE Trans. Commun., vol. 59, no. 1, pp. 116129, Jan. 2011.

[51] R. Rajashekar, K. V. S. Hari, and L. Hanzo, "Field extension code based dispersion matrices for coherently detected space-time shift keying," in Proc. IEEE GLOBECOM, Houston, TX, USA, Dec. 2011, pp. $1-5$.

[52] E. Basar, U. Aygolu, E. Panayirci, and H. V. Poor, "New trellis code design for spatial modulation," IEEE Trans. Wireless Commun., vol. 10, no. 8, pp. 2670-2680, Aug. 2011.

[53] P. Yang, Y. Xiao, Y. Yu, and S. Li, "Adaptive spatial modulation for wireless MIMO transmission systems," IEEE Commun. Lett., vol. 15, no. 6, pp. 602-604, Jun. 2011.

[54] H. A. Ngo, C. Xu, S. Sugiura, and L. Hanzo, "Space-time-frequency shift keying for dispersive channels," IEEE Signal Process. Lett., vol. 18, no. 3, pp. 177-180, Mar. 2011.

[55] M. D. Renzo, H. Haas, and P. Grant, "Spatial modulation for multipleantenna wireless systems: A survey," IEEE Commun. Mag., vol. 49, no. 11, pp. 182-191, Dec. 2011

[56] P. Som and A. Chockalingam, "Spatial modulation and space shift keying in single carrier communication," in Proc. IEEE 23rd Int. Symp. PIMRC, Sep. 2012, pp. 1962-1967.

[57] E. Basar, U. Aygolu, E. Panayirci, and H. V. Poor, "Super-orthogonal trellis-coded spatial modulation," IET Commun., vol. 6, no. 17 , pp. 2922-2932, Nov. 2012.

[58] P. Yang, Y. Xiao, L. Li, Q. Tang, and S. Li, "An improved matched-filter based detection algorithm for space-time shift keying systems," IEEE Signal Process. Lett., vol. 19, no. 5, pp. 271-274, May 2012.

[59] F. Babich, A. Crismani, M. Driusso, and L. Hanzo, "Design criteria and genetic algorithm aided optimization of three-stage-concatenated space-time shift keying systems," IEEE Signal Process. Lett., vol. 19, no. 8, pp. 543-546, Aug. 2012.

[60] R. Rajashekar, K. V. S. Hari, and L. Hanzo, "Structured dispersion matrices from division algebra codes for space-time shift keying," IEEE Signal Process. Lett., vol. 20, no. 4, pp. 371-374, Apr. 2013.

[61] H. A. Ngo and L. Hanzo, "Area spectral efficiency of soft-decision space-time-frequency shift-keying-aided slow-frequency-hopping multiple access," IEEE Trans. Veh. Technol., vol. 61, no. 3, pp. 1433-1439, Mar. 2012.

[62] P. Zhang, S. Chen, and L. Hanzo, "Differential space-time shift keyingaided successive-relay-assisted decode-and-forward cooperative multiuser CDMA," IEEE Trans. Veh. Technol., vol. 62, no. 5, pp. 2156-2169, Jun. 2013

[63] M. D. Renzo and H. Haas, "On transmit diversity for spatial modulation MIMO: Impact of spatial constellation diagram and shaping filters at the transmitter," IEEE Trans. Veh. Technol., vol. 62, no. 6, pp. 2507-2531, Jul. 2013.

[64] R. Rajashekar, K. V. S. Hari, and L. Hanzo, "Antenna selection in spatial modulation systems," IEEE Commun. Lett., vol. 17, no. 3, pp. 521-524, Mar. 2013.

[65] N. Serafimovski et al., "On the joint optimization of dispersion matrices and constellations for near-capacity irregular precoded space-time shift keying," IEEE Trans. Wireless Commun., vol. 12, no. 1, pp. 380-387, Jan. 2013 
[66] S. Sugiura and L. Hanzo, "On the joint optimization of dispersion matrices and constellations for near-capacity irregular precoded space-time shift keying," IEEE Trans. Wireless Commun., vol. 12, no. 1, pp. 380387, Jan. 2013.

[67] C. Xu, S. Sugiura, S. X. Ng, and L. Hanzo, "Spatial modulation and space-time shift keying: Optimal performance at a reduced detection complexity," IEEE Trans. Commun., vol. 61, no. 1, pp. 206-216, Jan. 2013.

[68] P. Yang, M. Di Renzo, Y. Xiao, S. Li, and L. Hanzo, "Design guidelines for spatial modulation," IEEE Commun. Surveys Tuts., to be published.

[69] M. I. Kadir, S. Chen, K. V. S. Hari, K. Giridhar, and L. Hanzo, "OFDMaided differential space-time shift keying using iterative soft multiplesymbol differential sphere decoding," IEEE Trans. Veh. Technol., vol. 63, no. 8, pp. 4102-4108, Oct. 2014.

[70] M. D. Renzo, H. Haas, A. Ghrayeb, S. Sugiura, and L. Hanzo, "Spatial modulation for generalized MIMO: Challenges, opportunities and implementation," Proc. IEEE, vol. 102, no. 1, pp. 56-103, Jan. 2014.

[71] S. X. Ng and L. Hanzo, "On the MIMO channel capacity of multidimensional signal sets," IEEE Trans. Veh. Technol., vol. 55, no. 2, pp. 528536, Mar. 2006.

[72] M. Jiang and L. Hanzo, "Unitary linear dispersion code design and optimization for MIMO communication systems," IEEE Signal Process. Lett., vol. 17, no. 5, pp. 497-500, May 2010.

[73] K. A. D. Jong, Evolutionary Computation-A Unified Approach. Cambridge, MA, USA: MIT Press, 2006.

[74] S. Sugiura, "Dispersion matrix optimization for space-time shift keying," IEEE Commun. Lett., vol. 15, no. 11, pp. 1152-1155, Nov. 2011.

[75] M. Patzold, Mobile Fading Channels. New York, NY, USA: Wiley, 2003.

[76] R. Steele and L. Hanzo, Mobile Radio Communications, 2nd ed. New York, NY, USA: Wiley, 1999.

[77] COST 207, "Digital land mobile radio communications, final report," Office Official Publ. Eur. Commun., Luxembourg City, Luxembourg, Tech. Rep., 1989.

[78] T. Hwang, C. Yang, G. Wu, S. Li, and G. Y. Li, "OFDM and its wireless applications: A survey," IEEE Trans. Veh. Technol., vol. 58, no. 4, pp. 1673-1694, May 2009.

[79] G. L. Stuber et al., "Broadband MIMO-OFDM wireless communications," Proc. IEEE, vol. 92, no. 2, pp. 271-294, Feb. 2004.

[80] H. Sampath, S. Talwar, J. Tellado, V. Erceg, and A. Paulraj, "A fourthgeneration MIMO-OFDM broadband wireless system: Design, performance, field trial results," IEEE Commun. Mag., vol. 40, no. 9, pp. 143149, Sep. 2002.

[81] H. Bolcskei, "MIMO-OFDM wireless systems: Basics, perspectives, challenges," IEEE Wireless Commun., vol. 13, no. 4, pp. 31-37, Aug. 2006.

[82] Radio Broadcasting Systems: Digital Audio Broadcasting (DAB) to Mobile, Portable, and Fixed Receivers, ETSI EN 300 401, May 1997.

[83] Digital Video Broadcasting: Framing Structure, Channel Coding, Modulation for Digital Terrestrial Television, ETSI EN 300 744, Aug. 1997.

[84] IEEE Standard for Wireless LAN Medium Access Control (MAC) and Physical (PHY) Layer Specifications, IEEE Std. 802.11, Nov. 1997.

[85] Radio Equipment and Systems, High PErformance Radio Local Area Network (HYPERLAN) Type 1, ETSI ETS 300 652, Oct. 1996.

[86] R. W. Chang, "Synthesis of band-limited orthogonal signals for multichannel data transmission," Bell Syst. Tech. J., vol. 45, no. 10, pp. 17751796, Dec. 1966.

[87] S. Weinstein and P. Ebert, "Data transmission by frequency-division multiplexing using the discrete Fourier transform," IEEE Trans. Commun. Technol., vol. COM-19, no. 5, pp. 628-634, Oct. 1971.

[88] L. Cimini, Jr., "Analysis and simulation of a digital mobile channel using orthogonal frequency division multiplexing," IEEE Trans. Commun., vol. COM-33, no. 7, pp. 665-675, Jul. 1985.

[89] M. Alard and R. Lassalle, "Principles of modulation and channel coding for digital broadcasting for mobile receivers," EBU Tech. Rev., no. 224, pp. 168-190, Aug. 1987

[90] R. S. Blum, G. L. Ye, J. H. Winters, and Y. Qing, "Improved space-time coding for MIMO-OFDM wireless communications," IEEE Trans. Commun., vol. 49, no. 11, pp. 1873-1878, Nov. 2001.

[91] R. W. Chang, "Orthogonal frequency division multiplexing," U.S. Patent 3488 445, Jan. 6, 1970.

[92] J. M. Cioffi, "A multicarrier primer," Amati Commun. Corp./Stanford Univ., Standford, CA, USA, ANSI T1E1.4 91-157, Nov. 1991.

[93] R. V. Nee and R. Prasad, OFDM for Wireless Multimedia Communications, 1st ed. Norwood, MA, USA: Artech House, 2000.
[94] L. Hanzo, M. Munster, B. J. Choi, and T. Keller, OFDM and MC-CDMA for Broadcasting Multi-User Communications, WLANs and Broadcasting. Hoboken, NJ, USA: Wiley, 2003.

[95] C. Williams, M. A. Beach, and S. Mclaughlin, "Robust OFDM timing synchronisation," Electron. Lett., vol. 41, no. 13, pp. 4-5, Jun. 2005.

[96] H. Chen, W. Gao, and D. Daut, "Spectrum sensing for OFDM systems employing pilot tones," IEEE Trans. Wireless Commun., vol. 8, no. 12, pp. 5862-5870, Dec. 2009.

[97] A. Sahin, I. Guvenc, and H. Arslan, "A survey on multicarrier communications: Prototype filters, lattice structures, implementation aspects," IEEE Commun. Surveys Tuts., vol. 16, no. 3, pp. 1312-1338, 2014.

[98] R. J. Piechocki, P. N. Fletcher, A. R. Nix, C. N. Canagarajah, and J. P. McGeehan, "Performance evaluation of BLAST-OFDM enhanced Hiperlan/2 using simulated and measured channel data," Electron. Lett., vol. 37, no. 18, pp. 1137-1139, May 2001

[99] H. Bolcskei, D. Gesbert, and A. J. Paulraj, "On the capacity of OFDMbased spatial multiplexing systems," IEEE Trans. Commun., vol. 50, no. 2, pp. 225-234, Feb. 2002.

[100] Y. Li, J. H. Winters, and N. R. Sollenberger, "MIMO-OFDM for wireless communications: Signal detection with enhanced channel estimation," IEEE Trans. Commun., vol. 50, no. 9, pp. 1471-1477, Sep. 2002.

[101] A. Stamoulis, S. N. Diggavi, and N. Al-Dhahir, "Intercarrier interference in MIMO OFDM," IEEE Trans. Signal Process., vol. 50, no. 10, pp. 2451-2464, Oct. 2002.

[102] H. El-Gamal, A. R. Hammons, Jr., Y. Liu, M. P. Fitz, and O. Y. Takeshita, "On the design of space-time and space-frequency codes for MIMO frequency-selective fading channels," IEEE Trans. Inf. Theory, vol. 49, no. 9, pp. 2277-2292, Sep. 2003

[103] J. H. Moon, Y. H. You, W. G. Jeon, K. W. Kwon, and H. K. Song, "Peak-to-average power control for multiple-antenna HIPERLAN/2 and IEEE802.11a systems," IEEE Trans. Consum. Electron., vol. 49, no. 4, pp. 1078-1083, Nov. 2003.

[104] D. Huang and K. Letaief, "Symbol-based space diversity for coded OFDM systems," IEEE Trans. Wireless Commun., vol. 3, no. 1, pp. 117 127, Jan. 2004.

[105] W. Su, Z. Safar, and K. J. R. Liu, "Full-rate full-diversity spacefrequency codes with optimum coding advantage," IEEE Trans. Inf. Theory, vol. 51, no. 1, pp. 229-249, Jan. 2005.

[106] M. Borgmann and H. Bolcskei, "Noncoherent space-frequency coded MIMO-OFDM,” IEEE J. Sel. Areas Commun., vol. 23, no. 9, pp. 17991810, Sep. 2005.

[107] T. H. Liew and L. Hanzo, "Space-time trellis and space-time block coding versus adaptive modulation and coding aided OFDM for wideband channels," IEEE Trans. Veh. Technol., vol. 55, no. 1, pp. 173-187, Jan. 2006.

[108] N. I. Miridakis and D. D. Vergados, "A survey on the successive interference cancellation performance for single-antenna and multiple-antenna OFDM systems," IEEE Commun. Surveys Tuts., vol. 15, no. 1, pp. 312 335,2013

[109] A. Al-Dweik, F. Kalbat, S. Muhaidat, O. Filio, and S. M. Ali, "Robust MIMO-OFDM system for frequency-selective mobile wireless channels," IEEE Trans. Veh. Technol., to be published.

[110] L.-L. Yang, Multicarrier Communications. Hoboken, NJ, USA: Wiley, Jan. 2009.

[111] T. Keller and L. Hanzo, "Adaptive multicarrier modulation: A convenient framework for time-frequency processing in wireless communications," Proc. IEEE, vol. 88, no. 5, pp. 611-640, May 2000.

[112] L. D’Orazio, C. Sacchi, M. Donelli, J. Louveaux, and L. Vandendorpe, "A near-optimum multiuser receiver for STBC MC-CDMA systems based on minimum conditional BER criterion and genetic algorithmassisted channel estimation," EURASIP J. Wireless Commun. Netw., vol. 2011, no. 1, p. 351 494, Mar. 2011. [Online]. Available: http://jwcn. eurasipjournals.com/content/2011/1/351494

[113] G. Fettweis, J. P. Linnartz, and N. Yee, "Multi-carrier CDMA in indoo wireless radio networks," in Proc. IEEE PIMRC, Yokohama, Japan, Sep. 1993, pp. 109-113.

[114] A. Chouly, A. Brajal, and S. Jourdan, "Orthogonal multicarrier techniques applied to direct sequence spread spectrum CDMA systems," in Proc. IEEE GLOBECOM, Houston, TX, USA, Nov./Dec. 1993, pp. 1723-1728.

[115] V. D. Silva and E. S. Sousa, "Performance of orthogonal CDMA codes for quasi-synchronous communication systems," in Proc. IEEE ICUPC, Ottawa, ON, Canada, Oct. 1993, pp. 995-999.

[116] L. Vandendorpe, "Multitone direct sequence CDMA system in an indoor wireless environment," in Proc. IEEE 1st Symp. Commun. Veh. Technol. Benelux, Delft, The Netherlands, Oct. 1993, pp. 4.1.1-4.1.8. 
[117] S. Hara and R. Prasad, "Overview of multi-carrier CDMA," IEEE Commun. Mag., vol. 35, no. 12, pp. 126-133, Dec. 1997.

[118] L.-L. Yang and L. Hanzo, "Multicarrier DS-CDMA: A multiple access scheme for ubiquitous broadband wireless communications," IEEE Commun. Mag., vol. 41, no. 10, pp. 116-124, Oct. 2003.

[119] L.-L. Yang, W. Hua, and L. Hanzo, "A multicarrier DS-CDMA system using both time-domain and frequency-domain spreading," in Proc. IEEE VTC - Fall, Orlando, FL, USA, Oct. 2003, vol. 4, pp. 2426-2430.

[120] L.-L. Yang, W. Hua, and L. Hanzo, "Multiuser detection in multicarrier CDMA systems employing both time-domain and frequency-domain spreading," in Proc. IEEE PIMRC, Beijing, China, Sep. 2003, vol. 2, pp. $1840-1844$

[121] C.-M. Yang, P.-H. Lin, G.-C. Yang, and W. C. Kwong, "2D orthogonal spreading codes for multicarrier DS-CDMA systems," in Proc. IEEE ICC, Anchorage, AK, USA, May 2003, vol. 5, pp. 3277-3281.

[122] G. Berardinelli, L. A. R. Temino, S. Frattasi, M. I. Rahman, and P. Mogensen, "OFDMA vs. SC-FDMA: Performance comparison in local area IMT-A scenarios," IEEE Wireless Commun., vol. 15, no. 5, pp. 64-72, Oct. 2008.

[123] N. Benvenuto, R. Dinis, D. Falconer, and S. Tomasin, "Single carrier modulation with nonlinear frequency domain equalization: An idea whose time has come-Again," Proc. IEEE, vol. 98, no. 1, pp. 69-96, Jan. 2010.

[124] T. Walzman and M. Schwartz, "Automatic equalization using the discrete frequency domain," IEEE Trans. Inf. Theory, vol. IT-19, no. 1, pp. 5968, Jan. 1973.

[125] H. Sari, G. Karam, and I. Jeanclaude, "Transmission techniques for digital terrestrial TV broadcasting," IEEE Commun. Mag., vol. 33, no. 2, pp. 100-109, Feb. 1995.

[126] D. Falconer, S. L. Ariyavisitakul, A. Benyamin-Seeyar, and B. Eidson, "Frequency domain equalization for single-carrier broadband wireless systems," IEEE Commun. Mag., vol. 40, no. 4, pp. 58-66, Apr. 2002.

[127] D. Falconer and S. L. Ariyavisitakul, "Broadband wireless using single carrier and frequency domain equalization," in Proc. 5th Int. Symp. WPMC, Honolulu, HI, USA, Oct. 2002, vol. 1, pp. 27-36.

[128] H. G. Myung, J. Lim, and D. J. Goodman, "Single carrier FDMA for uplink wireless transmission," IEEE Veh. Technol. Mag., vol. 1, no. 3, pp. 30-38, Sep. 2006.

[129] WINNER, "The WINNER II air interface: Refined multiple access concepts," Munich, Germany, IST-4-027756 WINNER II, D4.6.1, Tech. Rep., Nov. 2006.

[130] U. Sorger, I. D. Broeck, and M. Schnell, "Interleaved FDMA-A new spread-spectrum multiple-access scheme," in Proc. IEEE ICC, Atlanta, GA, USA, Jun. 1998, vol. 2, pp. 1013-1017.

[131] N. Benvenuto and S. Tomasin, "On the comparison between OFDM and single carrier modulation with a DFE using a frequency-domain feedforward filter," IEEE Trans. Commun., vol. 50, no. 6, pp. 947-955, Jun. 2002.

[132] A. Wilzeck, Q. Cai, M. Schiewer, and T. Kaiser, "Effect of multiple carrier frequency offsets in MIMO SC-FDMA systems," in Proc. Int. ITG/ IEEE Workshop Smart Antennas, Vienna, Austria, Feb. 2007, pp. 1-7.

[133] C. Ciochina and H. Sari, "A review of OFDMA and single-carrier FDMA," in Proc. EW, Lucca, Italy, Apr. 2010, pp. 706-710.

[134] V. Dalakas, P. T. Mathiopoulos, F. D. Cecca, and G. Gallinaro, "A comparative study between SC-FDMA and OFDMA schemes for satellite uplinks," IEEE Trans. Broadcast., vol. 58, no. 3, pp. 370-378, Sep. 2012.

[135] A. Ghosh, R. Ratasuk, B. Mondal, N. Mangalvedhe, and T. Thomas, "LTE-Advanced: Next-generation wireless broadband technology," IEEE Commun. Mag., vol. 17, no. 3, pp. 10-22, Jun. 2010.

[136] F. Boccardi et al., "Multiple-antenna techniques in LTE-Advanced," IEEE Commun. Mag., vol. 50, no. 3, pp. 114-121, Mar. 2012.

[137] M. I. Kadir, S. Chen, and L. Hanzo, "A reduced-complexity detector for OFDMA/SC-FDMA-aided space-time shift keying," in Proc. IEEE VTC - Fall, Las Vegas, NV, USA, Sep. 2013, pp. 1-5.

[138] L. Li and L. Hanzo, "Multiple-symbol differential sphere detection aided successive relaying in the cooperative DS-CDMA uplink," in Proc. IEEE WCNC, Cancun, Mexico, Mar. 2011, pp. 1875-1880.

[139] M. I. Kadir, "Co-located and distributed multicarrier space-time shift keying for wideband channels," Ph.D. dissertation, Univ. Southampton, Southampton, U.K., 2014.

[140] C. Xu, S. Sugiura, S. X. Ng, and L. Hanzo, "Reduced-complexity noncoherently detected differential space-time shift keying," IEEE Signal Process. Lett., vol. 18, no. 3, pp. 153-156, Mar. 2011.
[141] V. Pauli, L. Lampe, and J. Huber, "Differential space-frequency modulation and fast 2-D multiple-symbol differential detection for MIMOOFDM," IEEE Trans. Veh. Technol., vol. 57, no. 1, pp. 297-310, Jan. 2008.

[142] L. Wang, "Low-complexity near-optimum detection techniques for noncooperative and cooperative MIMO systems," Ph.D. dissertation, Univ. Southampton, Southampton, U.K., 2010.

[143] L. Hanzo, Y. Akhtman, L. Wang, and M. Jiang, MIMO-OFDM for LTE, WIFI and WIMAX: Coherent Versus Non-Coherent and Cooperative Turbo-Receivers. Hoboken, NJ, USA: Wiley, 2001.

[144] D. Divsalar and M. K. Simon, "Multiple-symbol differential detection of MPSK," IEEE Trans. Commun., vol. 38, no. 3, pp. 300-308, Mar. 1990

[145] L. Lampe, R. Schober, V. Pauli, and C. Windpassinger, "Multiple-symbol differential sphere decoding," IEEE Trans. Commun., vol. 53, no. 12, pp. 1981-1985, Dec. 2005.

[146] V. Pauli and L. Lampe, "On the complexity of sphere decoding for differential detection," IEEE Trans. Inf. Theory, vol. 53, no. 4, pp. 15951603, Apr. 2007

[147] V. Pauli and L. Lampe, "Tree-search multiple-symbol differential decoding for unitary space-time modulation," IEEE Trans. Commun., vol. 55, no. 8, pp. 1567-1576, Aug. 2007.

[148] V. Pauli, L. Lampe, and R. Schober, “"Turbo DPSK” using soft multiplesymbol differential sphere decoding," IEEE Trans. Inf. Theory, vol. 52, no. 4, pp. 1385-1398, Apr. 2006.

[149] E. Agrell, T. Eriksson, A. Vardy, and K. Zeger, "Closest point search in lattices," IEEE Trans. Inf. Theory, vol. 48, no. 8, pp. 2201-2214, Aug. 2002.

[150] T. Wiegand, N. Heidmann, and S. Paul, "Modified partial Euclidean distance for iterative tree-search MIMO detection," in Proc. IEEE PIMRC, Toronto, ON, Canada, Sep. 2011, pp. 1723-1727.

[151] R. C. Palat, A. Annamalai, and J. H. Reed, "Accurate bit-error-rate analysis of bandlimited cooperative OSTBC networks under timing synchronization errors," IEEE Trans. Veh. Technol., vol. 58, no. 5, pp. $2191-$ 2200, Jun. 2009

[152] X. Li, "Space-time coded multi-transmission among distributed transmitters without perfect synchronization," IEEE Signal Process. Lett., vol. 11, no. 12, pp. 948-951, Dec. 2004.

[153] Y. Li and X.-G. Xia, "A family of distributed space-time trellis codes with asynchronous cooperative diversity," IEEE Trans. Commun., vol. 55, no. 4, pp. 790-800, Apr. 2007.

[154] Z. Li and X.-G. Xia, "An Alamouti coded OFDM transmission for cooperative systems robust to both timing errors and frequency offsets," IEEE Trans. Wireless Commun., vol. 7, no. 5, pp. 1839-1844, May 2008.

[155] M. Sharp, A. Scaglione, and B. Sirkeci-Mergen, "Randomized cooperation in asynchronous dispersive links," IEEE Trans. Commun., vol. 57, no. 1, pp. 64-68, Jan. 2009.

[156] K. Wu, Y. J. Cheng, T. Djerafi, and W. Hong, "Substrate-integrated millimeter-wave and Terahertz antenna technology," Proc. IEEE, vol. 100, no. 7, pp. 2219-2232, Jul. 2012.

[157] J. Hoydis, S. ten Brink, and M. Debbah, "Massive MIMO in the UL/DL of cellular networks: How many antennas do we need?" IEEE J. Sel. Areas Commun., vol. 31, no. 2, pp. 160-171, Feb. 2013.

[158] L. Hanzo, M. El-Hajjar, and O. Alamri, "Near-capacity wireless transceivers and cooperative communications in the MIMO era: Evolution of standards, waveform design, future perspectives," Proc. IEEE, vol. 99, no. 8, pp. 1343-1385, Aug. 2011.

[159] R. Kudo, S. M. D. Armour, J. P. McGeehan, and M. Mizoguchi, "A channel state information feedback method for massive MIMO-OFDM," J. Commun. Netw., vol. 15, no. 4, pp. 352-361, Aug. 2013.

[160] E. G. Larsson, F. Tufvesson, O. Edfors, and T. L. Marzetta, "Massive MIMO for next generation wireless systems," IEEE Commun. Mag., vol. 52, no. 2, pp. 186-195, Feb. 2014.

[161] Q. Zhang, C. Yang, and A. Molisch, "Downlink base station cooperative transmission under limited-capacity backhaul," IEEE Trans. Wireless Commun., vol. 12, no. 8, pp. 3746-3759, Aug. 2013.

[162] P. Marsch and G. Fettweis, "Uplink CoMP under a constrained backhaul and imperfect channel knowledge," IEEE Trans. Wireless Commun., vol. 10, no. 6, pp. 1730-1742, Jun. 2011.

[163] D. Gesbert et al., "Multi-cell MIMO cooperative networks: A new look at interference," IEEE J. Sel. Areas Commun., vol. 28, no. 9, pp. 13801408, Dec. 2010.

[164] I. Hwang, C.-B. Chae, J. Lee, and R. W. Heath, Jr., "Multicell cooperative systems with multiple receive antennas," IEEE Trans. Wireless Commun., vol. 20, no. 1, pp. 50-58, Feb. 2013. 
[165] M.-S. Alouini and A. J. Goldsmith, "Area spectral efficiency of cellular mobile radio systems," IEEE Trans. Veh. Technol., vol. 48, no. 4, pp. 1047-1066, Jul. 1999.

[166] G. Ungerboeck, "Channel coding with multilevel/phase signals," IEEE Trans. Inf. Theory, vol. IT-28, no. 1, pp. 55-67, Jan. 1982.

[167] G. Ungerboeck, "Trellis-coded modulation with redundant signal sets-Part I: Introduction," IEEE Commun. Mag., vol. 25, no. 2, pp. 511, Feb. 1987.

[168] G. Ungerboeck, "Trellis-coded modulation with redundant signal sets-Part II: State of the art," IEEE Commun. Mag., vol. 25, no. 2, pp. 12-21, Feb. 1987.

[169] P. Robertson and T. Worz, "Bandwidth-efficient turbo trellis-coded modulation using punctured component codes," IEEE J. Sel. Areas Commun., vol. 16, no. 2, pp. 206-218, Feb. 1998

[170] G. Caire, G. Taricco, and E. Biglieri, "Bit-interleaved coded modulation," IEEE Trans. Inf. Theory, vol. 44, no. 3, pp. 927-946, May 1998.

[171] X. Li, A. Chindapol, and J. A. Ritcey, "Bit-interleaved coded modulation with iterative decoding and 8 PSK signaling," IEEE Trans. Commun., vol. 50, no. 8, pp. 1250-1257, Aug. 2002.

[172] L. Lampe and R. Schober, "Bit-interleaved coded differential space-time modulation," IEEE Trans. Commun., vol. 50, no. 9, pp. 1429-1439, Sep. 2002.

[173] C. H. Choi and G. H. Im, "Bit-interleaved coded multilevel modulation for single-carrier frequency-domain equalization," IEEE Commun. Lett., vol. 14, no. 3, pp. 193-195, Mar. 2010.

[174] R. Tee, R. G. Maunder, and L. Hanzo, "EXIT-chart aided near-capacity irregular bit-interleaved coded modulation design," IEEE Trans. Wireless Commun., vol. 8, no. 1, pp. 32-37, Jan. 2009.

[175] R. Ahlswede, N. Cai, S.-Y. Li, and R. W. Yeung, "Network information flow," IEEE Trans. Inf. Theory, vol. 46, no. 4, pp. 1204-1216, Jul. 2000.

[176] Z. Chen, B. Xia, Z. Hu, and H. Liu, "Design and analysis of multi-level physical-layer network coding for Gaussian two-way relay channels," IEEE Trans. Commun., vol. 62, no. 6, pp. 1803-1817, Jun. 2014.

[177] G. Bartoli, R. Fantacci, D. Marabissi, and R. Simoni, "Subcarriers suppression methods for OFDM systems with decode-and-forward network coding," IEEE Trans. Wireless Commun., vol. 12, no. 12, pp. 6034-6042, Dec. 2013.

[178] R. Bassoli, H. Marques, J. Rodriguez, K. W. Shum, and R. Tafazolli, "Network coding theory: A survey," IEEE Commun. Surveys Tuts., vol. 15, no. 4, pp. 1950-1978, 2013.

[179] Y. Kim and J. Kim, "A 2-D subcarrier allocation scheme for capacity enhancement in a clustered OFDM system," IEICE Trans., vol. 90-B, no. 7, pp. 1880-1883, Jul. 2007.

[180] W. K. Harold, "The Hungarian method for the assignment problem," Naval Res. Logist. Quart., vol. 2, no. 1/2, pp. 83-97, Mar. 1955.

[181] N. Y. Ermolova and B. Makarevitch, "Performance of practical subcarrier allocation schemes for OFDMA," in Proc. IEEE PIMRC, Athens, Greece, Sep. 2007, pp. 1-4.

[182] T. Liu, C. Yang, and L.-L. Yang, "A low-complexity subcarrierpower allocation scheme for frequency-division multiple-access systems," IEEE Trans. Wireless Commun., vol. 9, no. 5, pp. 1564-1570, May 2010.

[183] J. Lim, H. G. Myung, K. Oh, and D. J. Goodman, "Channel-dependent scheduling of uplink single carrier FDMA systems," in Proc. IEEE VTC - Fall, Montreal, QC, Canada, Sep. 2006, pp. 1-5.

[184] J. Zhang, L.-L. Yang, and L. Hanzo, "Power-efficient opportunistic amplify-and-forward single-relay aided multi-user SC-FDMA uplink," in Proc. IEEE VTC-Spring, Taipei, Taiwan, May 2010, pp. 1-5.

[185] J. Zhang, L.-L. Yang, and L. Hanzo, "Energy-efficient channeldependent cooperative relaying for the multiuser SC-FDMA uplink," IEEE Trans. Veh. Technol., vol. 60, no. 3, pp. 992-1004, Mar. 2011.

[186] D. Feng et al., "A survey of energy-efficient wireless communications," IEEE Commun. Surveys Tuts., vol. 15, no. 1, pp. 167-178, 2013.

[187] G. Li et al., "Energy-efficient wireless communications: Tutorial, survey, open issues," IEEE Trans. Wireless Commun., vol. 18, no. 6, pp. 28-35, Dec. 2011.

[188] L. Hanzo et al., "Wireless myths, realities, futures: From 3G/4G to optical and quantum wireless," Proc. IEEE, vol. 100, no. Special Centennial Issue, pp. 1853-1888, May 2012.

[189] T. S. Rappaport et al., "Millimeter wave mobile communications for 5G cellular: It will work!" IEEE Access, vol. 1, pp. 335-349, 2013.

[190] F. Rusek et al., "Scaling up MIMO: Opportunities and challenges with very large arrays," IEEE Signal Process. Mag., vol. 30, no. 1, pp. 40-60, Jan. 2013.
[191] G. Y. Li, J. Niu, D. Lee, J. Fan, and Y. Fu, "Multi-cell coordinated scheduling and MIMO in LTE," IEEE Commun. Surveys Tuts., vol. 16, no. 2, pp. 761-775, 2014.

[192] M. N. Tehrani, M. Uysal, and H. Yanikomeroglu, "Device-to-device communication in $5 \mathrm{G}$ cellular networks: Challenges, solutions, future directions," IEEE Commun. Mag., vol. 52, no. 5, pp. 86-92, May 2014.

[193] D. Feng et al., "Device-to-device communications in cellular networks," IEEE Commun. Mag., vol. 52, no. 4, pp. 49-55, Apr. 2014.

[194] V. Jungnickel et al., "The role of small cells, coordinated multipoint, massive MIMO in 5G," IEEE Commun. Mag., vol. 52, no. 5, pp. 44-51, May 2014.

[195] W. H. Chin, Z. Fan, and R. Haines, "Emerging technologies and research challenges for 5G wireless networks," IEEE Wireless Commun., vol. 21, no. 2, pp. 106-112, Apr. 2014.

[196] C.-X. Wang et al., "Cellular architecture and key technologies for 5G wireless communication networks," IEEE Commun. Mag., vol. 52, no. 2 , pp. 122-130, Feb. 2014

[197] T. S. Rappaport, J. N. Murdock, and F. Gutierrez, "State of the art in 60-GHz integrated circuits and systems for wireless communications," Proc. IEEE, vol. 99, no. 8, pp. 1390-1436, Aug. 2011.

[198] E. Hossain, M. Rasti, H. Tabassum, and A. Abdelnasser, "Evolution toward 5G multi-tier cellular wireless networks: An interference management perspective," IEEE Wireless Commun., vol. 21, no. 3, pp. 118127, Jun. 2014

[199] A. Osseiran et al., "Scenarios for 5G mobile and wireless communications: The vision of the METIS project," IEEE Commun. Mag., vol. 52, no. 5, pp. 26-35, May 2014.

[200] H. Q. Ngo, E. G. Larsson, and T. Marzetta, "Energy and spectral efficiency of very large multiuser MIMO systems," IEEE Trans. Commun., vol. 61, no. 4, pp. 1436-1449, Apr. 2013.

[201] F. Boccardi, R. W. Heath, A. Lozano, T. L. Marzetta, and P. Popovski, "Five disruptive technology directions for 5G," IEEE Commun. Mag., vol. 52, no. 2, pp. 74-80, Feb. 2014.

[202] T. Bai, A. Alkhateeb, and R. W. Heath, Jr., "Coverage and capacity of millimeter-wave cellular networks," IEEE Commun. Mag., vol. 52, no. 9 , pp. 70-77, Sep. 2014

[203] A. L. Swindlehurst, E. Ayanoglu, P. Heydari, and F. Capolino, "Millimeter-wave massive MIMO: The next wireless revolution?" IEEE Commun. Mag., vol. 52, no. 9, pp. 56-62, Sep. 2014.

[204] W. Roh et al., "Millimeter-wave beamforming as an enabling technology for 5G cellular communications: Theoretical feasibility and prototype results," IEEE Commun. Mag., vol. 52, no. 2, pp. 106-113, Feb. 2014

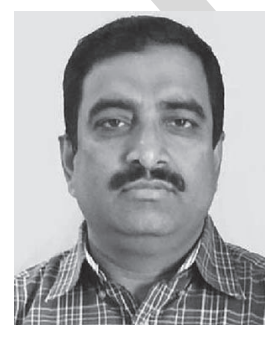

Mohammad Ismat Kadir received the B.Sc.(Eng.) degree (first class) in electrical and electronic engineering and the M.Sc.(Eng.) degree in computer engineering from Bangladesh University of Engineering and Technology, Dhaka, Bangladesh, in 1992 and 1999, respectively, and the Ph.D. degree in electronics and electrical engineering from the University of Southampton, Southampton, U.K., in 2014 .

$\mathrm{He}$ is currently a Faculty Member in the Electronics and Communication Engineering Discipline, Khulna University, Khulna, Bangladesh. His research interests include multicarrier systems, space-time coding, cooperative communications, millimeterwave communications, and heterogeneous networks. Dr. Kadir was a recipient of the Commonwealth Scholarship from the Commonwealth Scholarship Commission, U.K. 


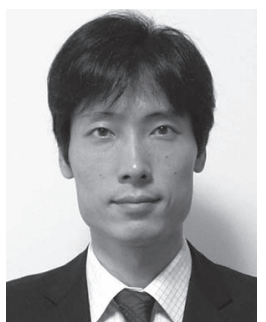

Shinya Sugiura (M'06-SM'12) received the B.S. and M.S. degrees in aeronautics and astronautics from Kyoto University, Kyoto, Japan, in 2002 and 2004, respectively, and the Ph.D. degree in electronics and electrical engineering from the University of Southampton, Southampton, U.K., in 2010.

From 2004 to 2012, he was a Research Scientist with Toyota Central Research and Development Laboratories, Inc., Aichi, Japan. Since January 2013, he has been an Associate Professor with the Department of Computer and Information Sciences, Tokyo University of Agriculture and Technology, Tokyo, Japan, where he heads the Wireless Communications Research Group. He has authored or coauthored over 60 refereed research publications, including 37 IEEE journal and magazine papers. His research has covered a range of areas in wireless communications, networking, signal processing, and antenna design. Dr. Sugiura was the recipient of numerous awards, including the 28th Telecom System Technology Award from the Telecommunications Advancement Foundation in 2013, the 6th IEEE Communications Society Asia-Pacific Outstanding Young Researcher Award in 2011, the 13th Ericsson Young Scientist Award in 2011, and the 2008 IEEE Antennas and Propagation Society Japan Chapter Young Engineer Award. He was also certified as an Exemplary Reviewer of IEEE COMMUNICATIONS LETTERS in 2013 and 2014.

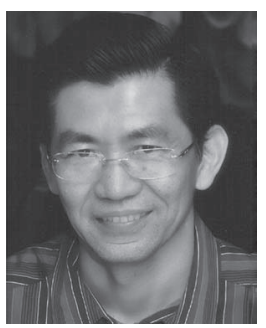

Sheng Chen (M'90-SM'97-F'08) received the B.Eng. degree in control engineering from East China Petroleum Institute, Dongying, China, in 1982, the Ph.D. degree in control engineering from the City University, London, U.K., in 1986, and the D.Sc. degree from the University of Southampton, Southampton, U.K., in 2005.

From 1986 to 1999 , he held research and academic appointments at the Universities of Sheffield, Edinburgh, and Portsmouth, all in U.K. Since 1999, he has been with School of Electronics and Computer Science, University of Southampton, where he currently holds the post of Professor in Intelligent Systems and Signal Processing. He has published over 500 research papers. His research interests include adaptive signal processing, wireless communications, modeling and identification of nonlinear systems, neural network and machine learning, intelligent control system design, evolutionary computation methods, and optimization.

Dr. Chen is a Fellow of the Institute of Engineering and Technology and the U.K. Royal Academy of Engineering. He is a Distinguished Adjunct Professor at King Abdulaziz University, Jeddah, Saudi Arabia, and an ISI Highly Cited Researcher in Engineering (March 2004).

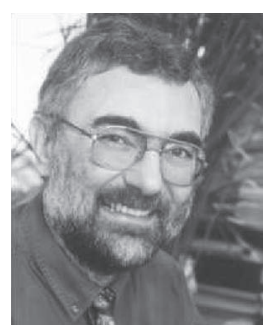

Lajos Hanzo (M'91-SM'92-F'04) received the M.S. degree in electronics and the Ph.D. degree from the Technical University of Budapest, Budapest, Hungary, in 1976 and 1983, respectively; the D.Sc. degree from the University of Southampton, Southampton, U.K., in 2004; and the "Doctor Honoris Causa" degree from the Technical University of Budapest in 2009. During his 38-year career in telecommunications, he has held various research and academic posts in Hungary, Germany, and the U.K. Since 1986, he has been with the School of Electronics and Computer Science, University of Southampton, where he holds the Chair in Telecommunications. He is currently directing a 100-strong academic research team, working on a range of research projects in the field of wireless multimedia communications sponsored by industry, the Engineering and Physical Sciences Research Council U.K., the European Research Council's Advanced Fellow Grant, and the Royal Society's Wolfson Research Merit Award. During 2008-2012, he was a Chaired Professor at Tsinghua University, Beijing. He is an enthusiastic supporter of industrial and academic liaison and offers a range of industrial courses. He has successfully supervised about 100 Ph.D. students, coauthored 20 John Wiley/IEEE Press books on mobile radio communications totaling in excess of 10000 pages, and published more than 1400 research entries at IEEE Xplore. His research is funded by the European Research Council's Senior Research Fellow Grant. Dr. Hanzo is a Fellow of the Royal Academy of Engineering, the Institution of Engineering and Technology, and the European Association for Signal Processing. He is also a Governor of the IEEE Vehicular Technology Society. During 2008-2012, he was the Editor-in-Chief of the IEEE Press. He has served as the Technical Program Committee Chair and the General Chair of IEEE conferences, has presented keynote lectures, and has been awarded a number of distinctions. He has more than 20000 citations. For further information on research in progress and associated publications, please refer to http://www-mobile.ecs.soton.ac.uk. 